\title{
Die Biomineralisation von Silica: \\ Langkettige Polyamine und Aminolipide als selbstorganisierende Template für biomimetische Präzipitationen
}

\author{
Dissertation \\ zur Erlangung des Doktorgrades \\ der Mathematisch-Naturwissenschaftlichen Fakultäten \\ der Georg-August-Universität zu Göttingen
}

\author{
vorgelegt von \\ Anja Christina Bernecker \\ aus Neuburg a.d. Donau
}

Göttingen 2009 
D 7

Referentin: Prof. Dr. Claudia Steinem

Korreferent: Prof. Dr. Franc Meyer

Tag der mündlichen Prüfung: 29.10.2009 
Die vorliegende Arbeit wurde an der Universität Göttingen, Fakultät für Chemie, Institut für Organische und Biomolekulare Chemie, in der Zeit von März 2006 bis September 2009 unter Anleitung von Frau Prof. Dr. C. Steinem angefertigt. 

Meiner Familie

Was wir wissen, ist ein Tropfen, was wir nicht wissen, ein Ozean. 

Abstract. Long-chain polyamines are thought to play an important role in the formation of the hierarchically structured silica cell walls of diatoms. We determined the influence of structural variations such as chain-length, hydrophobic spacer and $N$-methylation pattern of polyamines on silica precipitation reactions. Synthetic polyamines with well-defined structures and chain-lengths similar to those found in diatoms were used. These polyamines were shown to induce the formation of hollow silica spheres (diameters: $50-160 \mathrm{~nm}$ ) with narrow size distributions upon addition of monosilicic acid in the presence of phosphate ions, within ten minutes of addition. The silica shell thicknesses were observed to range from $20-60 \mathrm{~nm}$. The amount of silica, the size of the spheres and their morphology were strongly dependent on the polyamine structure. With increasing chain-length, length of the alkyl spacer and $N$-methylation, the amount of silica increases and the particle diameters also increase. Theoretical estimation of basicity and hydrophobicity of the polyamines shows a correlation that can be explained by the aggregation and phase-separation of polyamines.

A second topic that was investigated, was the development of self-assembly systems which can be used as templates for biomimetic silica syntheses. Synthetic amino compounds with hydrophobic anchor groups, so-called aminolipids, exhibit properties suitable for the formation of lamellar aggregates. These aminolipids are capable of inducing rapid synthesis of silica on their surfaces. Silica precipitation reactions were observed to occur on vesicles as well as planar lipid layers.

In the case of aminolipids, the driving force for aggregation is the hydrophobic interaction of the alkyl chains. The significance of phase separation and accumulation of positive charges on surfaces for the biomimetic formation of silica is emphasized by the fact that in this case silica polymerization takes place in the absence of phosphate ions.

Vesicles of aminolipids were used to examine the influence of $N$-methylation on the precipitation reaction. We found a correlation between surface charge and the amount of silica formed. Furthermore, silica films with a thickness of less than ten nanometers were synthesized on the surface of planar lipid layers. They consist of interconnected silica spheres whose nanostructures resemble those of diatom cell walls. Finally, a basis for the formation of structured planar silica films was established using phase-separated membrane domains from a binary lipid system consisting of a lysinol lipid and a glycolipid. 



\section{Inhaltsverzeichnis}

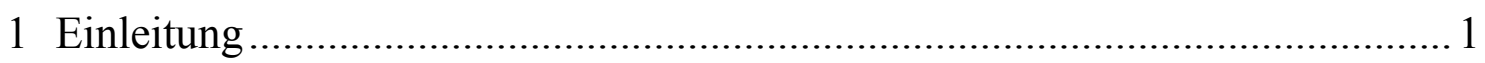

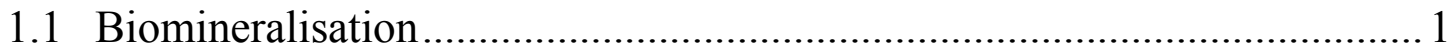

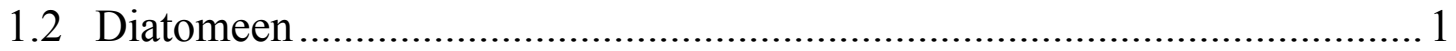

1.3 Chemische Grundlagen der Silicapolymerisation .................................... 5

1.4 Komponenten und Kontrolle der Silicabildung in Diatomeen .................... 8

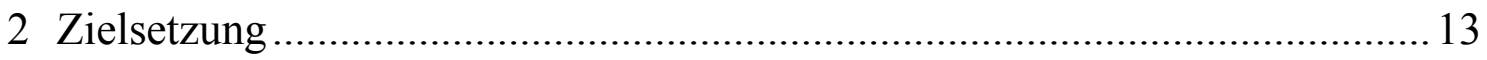

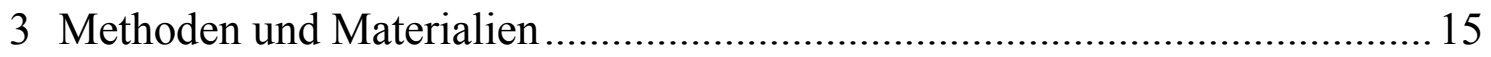

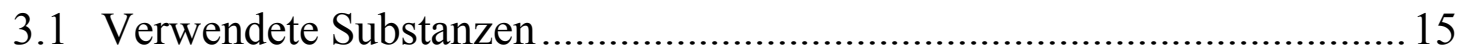

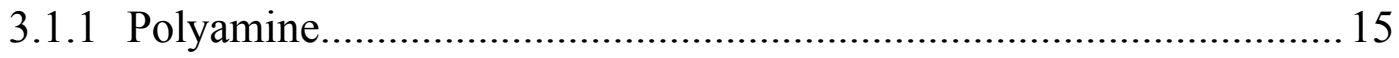

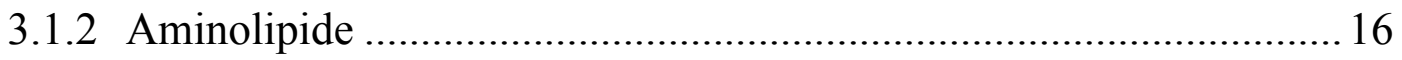

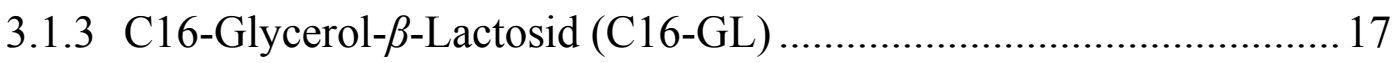

3.1.4 1,2-Dipalmitoyl-sn-glycero-3-phosphatidylcholin (DPPC)............... 18

3.1.5 Fluoreszenzsonden ...................................................................... 19

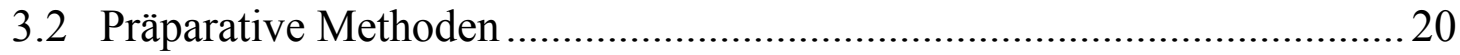

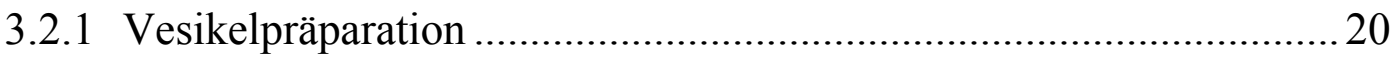

3.2.2 Herstellung von Lipiddoppelschichten mittels Spreiten von

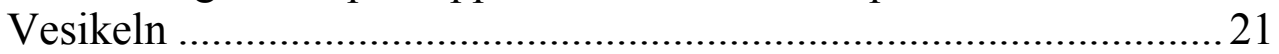

3.2.3 Herstellung von Lipiddoppelschichten mittels Langmuir-

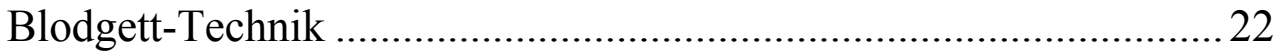

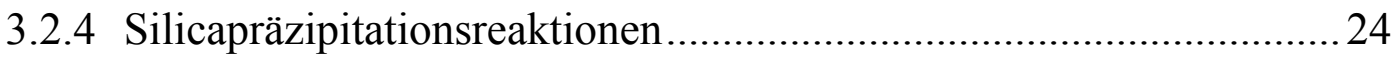

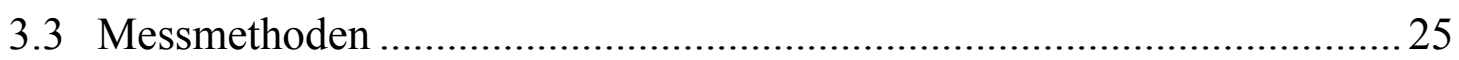

3.3.1 $\beta$-Molybdatmethode .................................................................... 25

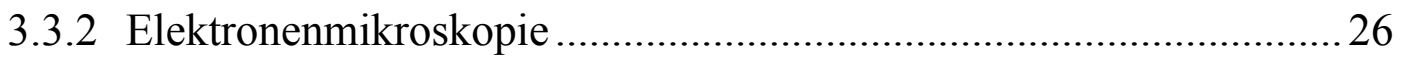

3.3.3 Dynamische Lichtstreuung ............................................................. 31

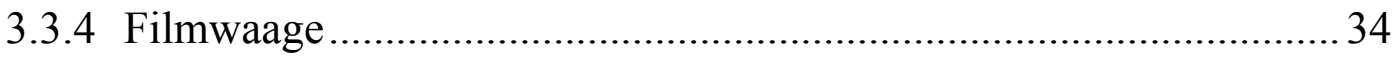

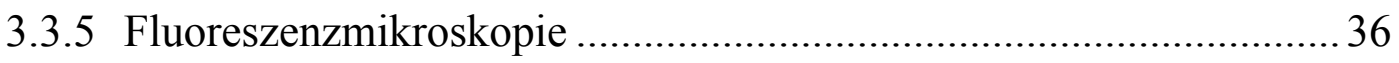

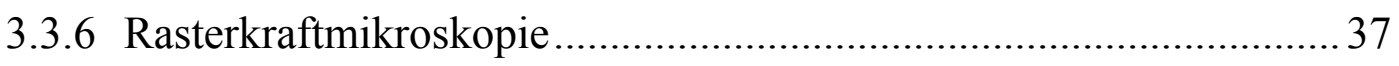

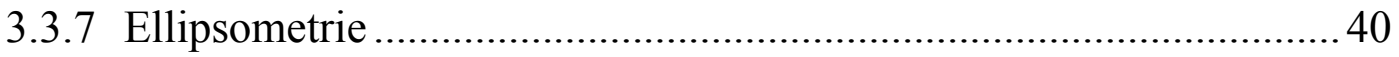

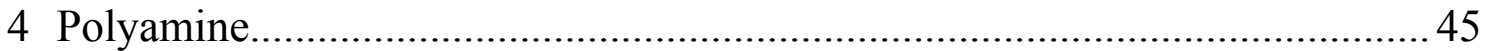

4.1 Strukturen und Eigenschaften der verwendeten Polyamine ....................... 45

4.2 Einfluss der Struktur von Polyaminen auf Präzipitationsreaktionen.......... 46

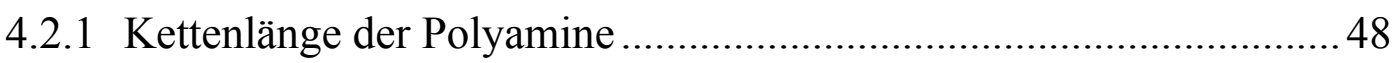




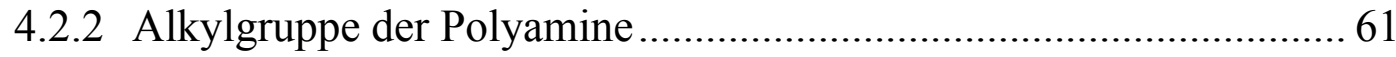

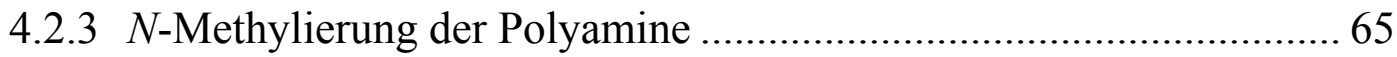

4.3 Einfluss von Phosphationen auf Präzipitationsreaktionen .......................... 66

4.4 Untersuchungen zur Reaktionsgeschwindigkeit....................................... 73

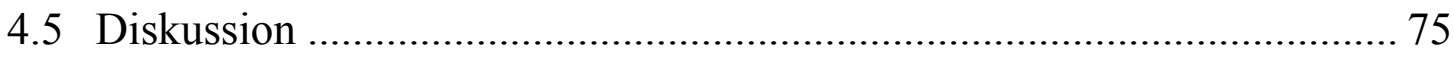

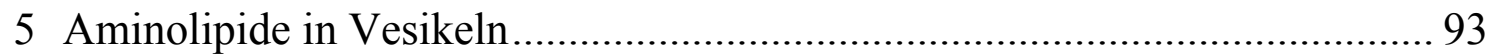

5.1 Strukturen und Eigenschaften der Aminolipide ........................................ 94

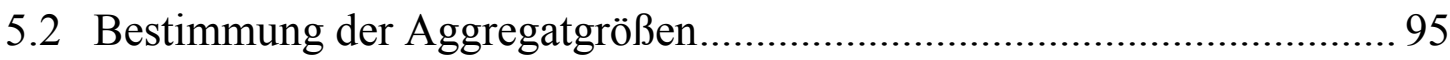

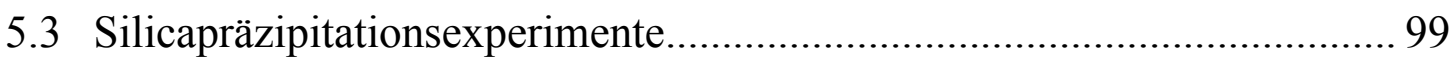

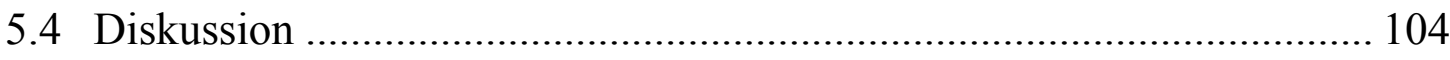

6 Aminolipide in planaren Membranen...................................................... 117

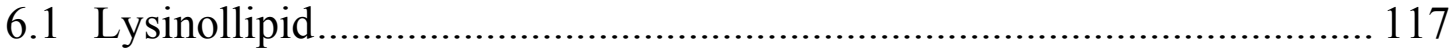

6.1.1 Charakterisierung der festkörperunterstützten Doppelschichten..... 117

6.1.2 Präzipitationsreaktionen an planaren Lysinollipiddoppelschichten 123

6.2 Das System Lysinollipid/C16-Glycerol- $\beta$-Lactosid................................. 132

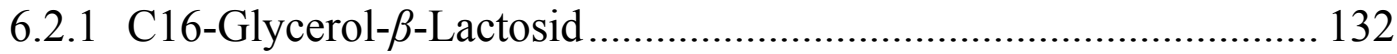

6.2.2 Lysinollipid/C16-Glycerol- $\beta$-Lactosid-Monoschichten an der Grenzfläche Wasser/Luft .......................................................... 133

6.2.3 Charakterisierung von festkörperunterstützten Lysinollipid/C16-

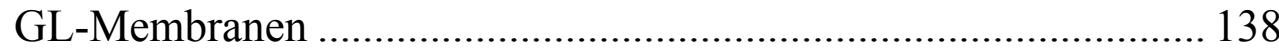

6.2.4 Präzipitationsreaktionen an festkörperunterstützten Lysinollipid/C16-GL-Membranen................................................ 140

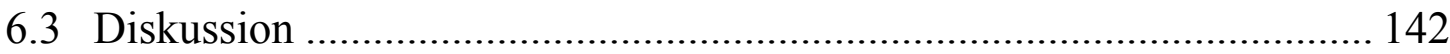

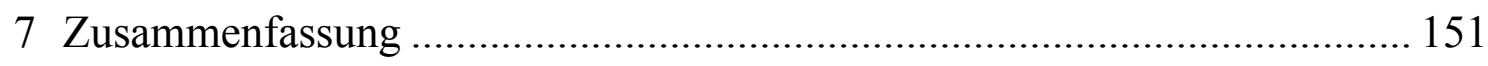

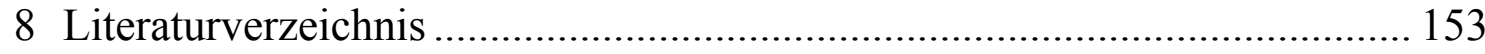

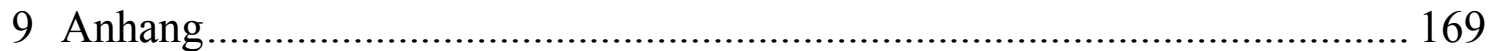

9.1 Berechnungen zum Säure-Base-Verhalten der Polyamine und

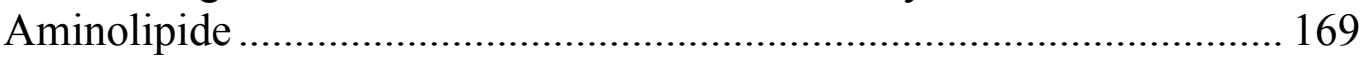

9.2 Zur Schichtdickenbestimmung mittels Ellipsometrie verwendete

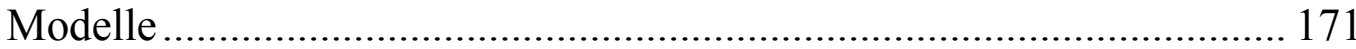

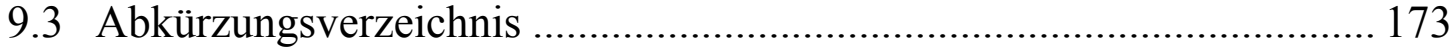

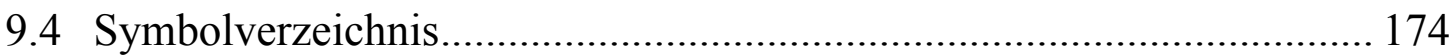

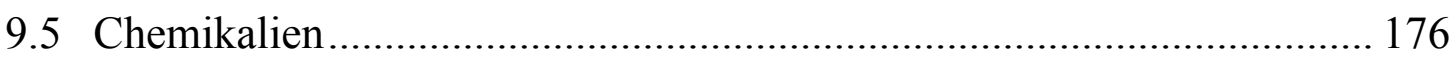

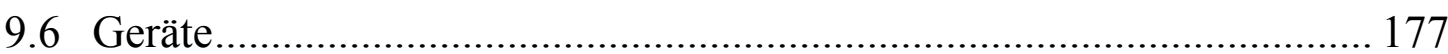






\section{Einleitung}

\subsection{Biomineralisation}

Als Biomineralisation wird die Bildung anorganischer Mineralien in Organismen bezeichnet. $^{1,2}$ Es ist ein weitverbreitetes Phänomen, das im gesamten Tier- und Pflanzenreich vom Prokaryoten bis zum Menschen hin zu finden ist. ${ }^{3}$ Über sechzig Biomineralien sind bekannt, unter denen Calciumcarbonat, Calciumphosphat und wasserhaltiges, amorphes Siliciumdioxid die häufigsten darstellen. ${ }^{4}$ Die Eigenschaften und Strukturen der zumeist auf mehreren Ebenen hierarchisch organisierten organischanorganischen Kompositmaterialien ${ }^{5}$ sind an die Aufgaben im jeweiligen Organismus angepasst. Ein besonderes Augenmerk ist hierbei auf Diatomeen gerichtet, die zusammen mit anderen marinen Organismen wie Silicoflagellaten, Radiolarien und Schwämmen durch die Bildung ihrer Kieselsäureskelette jährlich mit 6,7 Gigatonnen eine beachtliche Menge an Silicium biogen umsetzen. ${ }^{6,7}$ Die aus amorphem Silica bestehenden Zellwände dieser einzelligen eukaryotischen Algen beeindrucken durch ihre kunstvollen und aufwendigen Strukturen, die im Nano- wie im Mikromaßstab hoch differenziert sind. Da Biosilica in Diatomeen zudem unter physiologischen Bedingungen entsteht, ${ }^{8}$ während geochemische und industrielle Synthesen von Silica zumeist unter extremen Bedingungen bezüglich $\mathrm{pH}-$ Wert, Temperatur und Druck stattfinden, ${ }^{6}$ ist es von besonderem Interesse, die molekularen Vorgänge, die zu seiner Bildung führen, zu verstehen. Dabei umfasst die Erforschung der Biomineralisation viele wissenschaftliche Fachrichtungen, unter anderem Biochemie, Biologie, Geologie, anorganische Chemie sowie Molekularbiologie. ${ }^{5}$

\subsection{Diatomeen}

Seit ihrem Auftreten (älteste fossile Funde sind etwa 185 Millionen Jahre alt ${ }^{9}$ ) haben sich die Diatomeen (Bacillariophyta, Kieselalgen) mit bis zu 200000 Arten $^{10,11}$ zur speziesreichsten Gruppe eukaryotischer Algen entwickelt. Sie bevölkern nahezu alle Wasserhabitate und sind extrem wichtig für den biologischen Kohlenstoff- und SiliciumKreislauf. $^{3}$ Es wird geschätzt, dass die Einzeller für $25 \%$ der biologischen Nettoprimärproduktion verantwortlich sind. ${ }^{12}$ Eine Hypothese besagt, dass der evolutionäre Erfolg der Diatomeen unter anderem auf ihren Silicazellwänden beruht, da deren Bildung zum einen energetisch weniger aufwendig sein soll, als die von organischen ${ }^{13}$ Zellwänden 
und sie zum anderen besseren Schutz gewährleisten sollen. ${ }^{14}$ Es konnte gezeigt werden, dass die Zellwände der Diatomeen eine mechanische Stabilität aufweisen, die ausreichend ist, um Angriffen potenzieller Räuber zu widerstehen. ${ }^{15}$ Weiterhin wurde vorgeschlagen, dass das Silica in den Kieselalgen als pH-Puffer fungieren könnte. ${ }^{16}$

Die Bacillariophyta sind nach ihrer Morphologie traditionell in zwei Gruppen unterteilt, die radiärsymmetrischen Centrales (z.B. Thalassiosira pseudonana, Coscinodiscus sp., Aulacodiscus sp.oder Amphitetras sp.) und die lang gestreckten bilateral-symmetrischen Pennales (z. B. Cylindrotheca fusiformis, Didymosphenia sp. oder Podocystis sp.). In Abbildung 1.1 sind rasterelektronische Aufnahmen verschiedener Spezies dargestellt. Die Exoskelette der Diatomeen, genannt Frustula, werden oft als Glashaus oder Glasbox bezeichnet. Sie offenbaren ästhetische filigrane Feinstrukturen mit hierarchischem Aufbau.
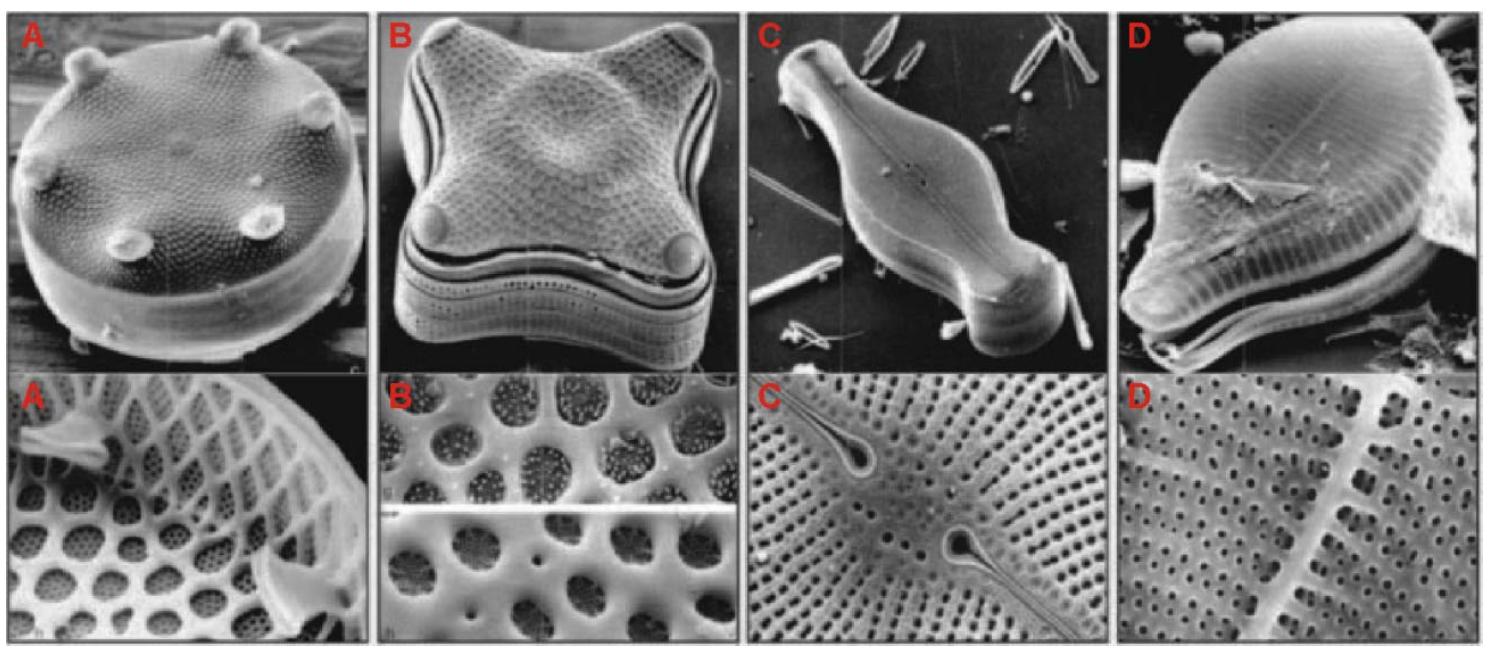

Abbildung 1.1 Rasterelektronenmikroskopische Aufnahmen der Zellwände verschiedener Diatomeenspezies: A Aulacodiscus sp. B Amphitetras sp. C Didymosphenia sp. D Podocystis sp., entnommen von Kröger ${ }^{3}$.

Die Silicazellwände der Diatomeen sind von einer organischen Hülle umgeben, die wahrscheinlich vor Desilifizierung schützt. ${ }^{17}$ Der Aufbau des Frustulums aus den einzelnen Komponenten folgt bei allen Diatomeen einem festen Bauplan (Abbildung 1.2). ${ }^{18}$ Man unterscheidet zwei Schalen, die größere Epitheka und die kleinere Hypotheka, die wie die zwei Teile einer Petrischale ineinander greifen. Jede Theka ist aus einer Valva und mehreren Gürtelbändern zusammengesetzt. Die Gürtelbänder, die sich im überlappenden Bereich befinden, werden Pleuralbänder genannt. 


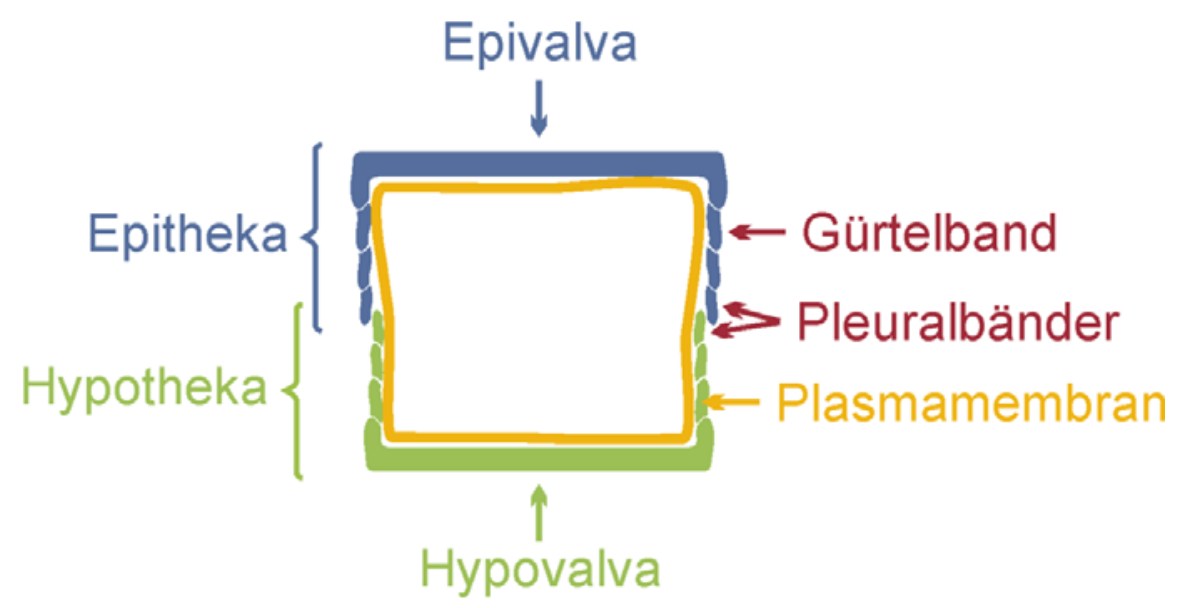

Abbildung 1.2 Schematische Darstellung des allgemeinen Aufbaus eines Diatomeen-Frustulums.

Während der dominierenden asexuellen Fortpflanzung teilt sich die Mutterzelle im Bereich der Pleuralbänder in zwei Tochterzellen (Abbildung 1.3). Jede der beiden neu entstehenden Zellen übernimmt eine Theka der Mutterzelle. In dieser Phase werden zwei neue Valven entwickelt, die den Tochterzellen jeweils als Hypovalva dienen. Seit langem ist bekannt, dass die Silicapolymerisation in spezialisierten membrangebundenen Vesikeln, den so genannten silica deposition vesicles (SDV), ${ }^{19}$ stattfindet, auch wenn diese Organellen noch nicht isoliert und charakterisiert werden konnten. Bekannt ist allerdings, dass der Membran, die das SDV umgibt (Silicalemma), ein Potential anliegt und dass das SDVLumen einen leicht sauren $\mathrm{pH}-$ Wert aufweist. ${ }^{20,21}$

Nach Bildung der jeweiligen Valva werden diese durch Exozytose des SDV an die Oberfläche der Zelle gebracht. Im Anschluss wird das Zellvolumen durch die Synthese von Gürtelbändern in eigens dafür vorgesehenen SDVs während der Interphase vergrößert. Über den intrazellulären Transport von Kieselsäure ist noch nicht viel bekannt, jedoch wurden spezifische Kieselsäuretransportproteine (silicon transporter, SIT) gefunden, die die Aufnahme von Kieselsäure in Form von $\mathrm{Si}(\mathrm{OH})_{4}{ }^{22}$ in die Zelle bewerkstelligen. ${ }^{23-25}$ Bezüglich der Ausbildung der Struktur der Silicazellwand wurden Simulationen ${ }^{26}$ durchgeführt und es wurden mehrere Hypothesen aufgestellt, die sich auf verschiedene Ebenen beziehen. Basierend auf Beobachtungen der Zwischenstufen und der endgültigen Strukturen wurden Modelle entwickelt, die sich mit der Bildung der größeren Strukturen auf der Mikroskala, wie die Gesamtform und die Valvaform, sowie denen auf der Mesoskala beschäftigen. ${ }^{17,27}$ Es wird angenommen, dass das Zytoskelett ${ }^{28}$ sowie eine Reihe anderer Organellen wie Mitochondrien oder Endoplasmatisches Retikulum ${ }^{13}$ das SDV formen und somit die äußere Form der sich bildenden Zellwand dirigieren. Die Formation der Feinstrukturen auf der Nanoskala wird zum einen über diffusionslimitierte Aggregation 
von $\mathrm{SiO}_{2}$-Nanopartikeln ${ }^{29,30}$ erklärt. Zum anderen wurden nach der Entdeckung einiger an der Silicabildung beteiligter organischer Komponenten auf self-assembly und Phasenseparation basierende Theorien vorgeschlagen, die versuchen, die Entstehung der Feinstrukturen zu erklären. Silaffinen und Polyaminen (siehe unten) werden Struktur kontrollierende Funktionen zugeschrieben, wobei auch in diesem Feld verschiedene Meinungen über die Mechanismen vorherrschen (vergleiche mit Kapitel 4.5). ${ }^{31-33}$

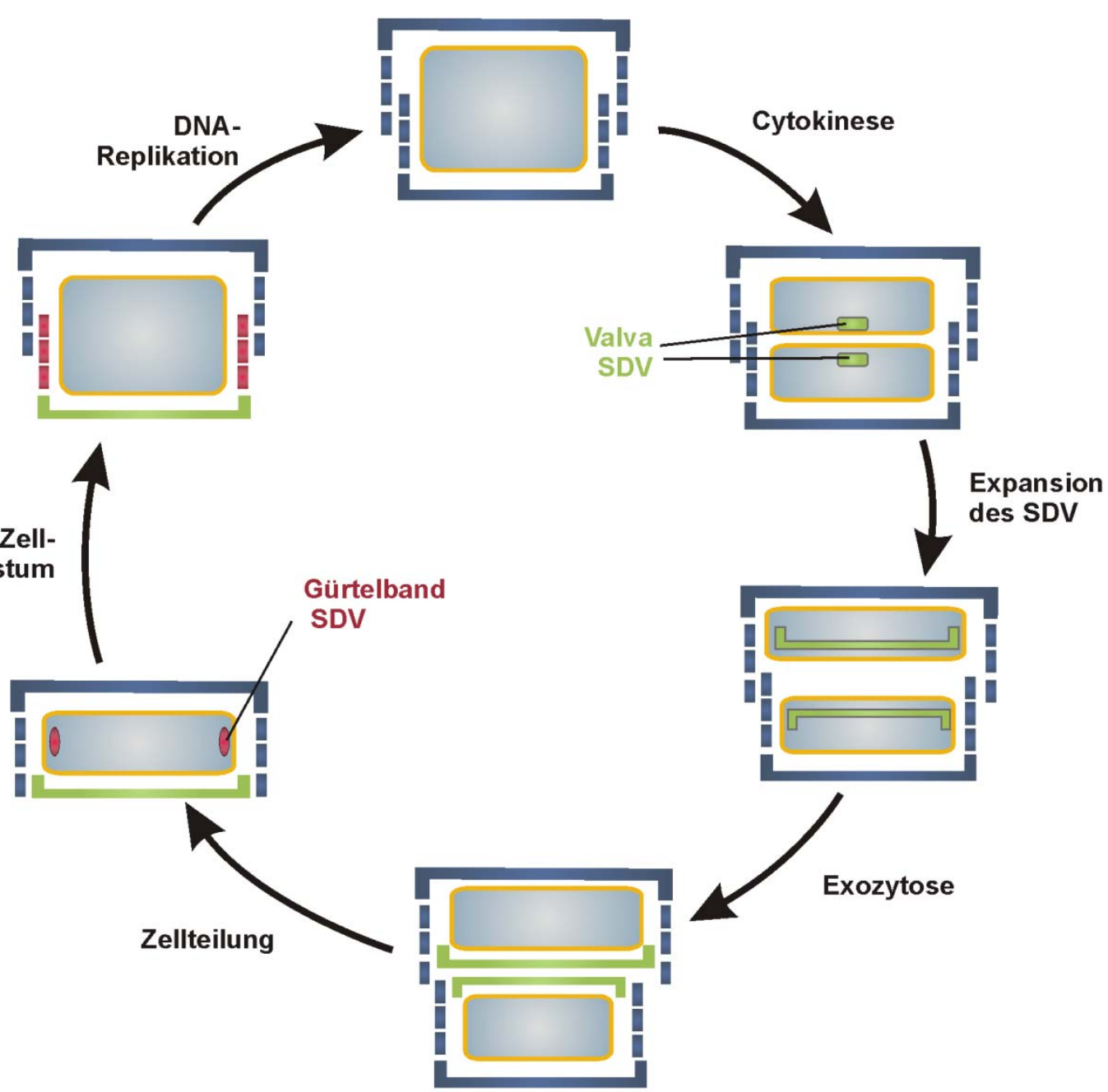

Abbildung 1.3 Schematische Darstellung des Zellzyklus von Diatomeen nach Kröger ${ }^{34}$.

Um ein besseres Verständnis für diese Modelle und die Vorgänge in den Diatomeen zu erhalten sowie die im Rahmen dieser Arbeiten gewonnenen Erkenntnisse interpretieren zu können, wird zunächst ein Überblick über die Silicachemie gegeben. 


\subsection{Chemische Grundlagen der Silicapolymerisation}

Die Eigenschaften von verschiedenen Kieselsäurespezies und ihr Reaktionsverhalten sind weit erforscht, auch wenn sie ein komplexes Thema darstellen und noch nicht vollständig verstanden sind. ${ }^{35-38}$ Monomere Kieselsäure $\mathrm{Si}(\mathrm{OH})_{4}$ ist bei $25{ }^{\circ} \mathrm{C}$ unterhalb einer Konzentration von $1 \mathrm{mM}$ in Wasser stabil, liegen höhere Konzentrationen vor, findet eine rasche Polymerisation statt. Im Gegensatz zu pH-Werten größer 7, wo die Polymerisationsprozesse sehr schnell ablaufen, lassen sich im Bereich von $\mathrm{pH} 2$ - 7 einzelne Schritte unterscheiden (Abbildung 1.4). Zunächst werden Dimere, Trimere bis hin zu Dodekameren gebildet. Mit zunehmender Substitution der Kieselsäure nimmt der $\mathrm{pK}_{\mathrm{s}}$-Wert der Silanolgruppe ab. Während dieser für $\mathrm{Si}(\mathrm{OH})_{4}$ bei 9,7 liegt, beträgt er im Fall höher polymerisierter Spezies 6,7; die Acidität steigt also mit der Anzahl an Siloxanbindungen. Aus diesem Grund liegen in Oligomeren mehr Hydroxygruppen dissoziiert in Form von Silanolatanionen vor. Dadurch wird die Polymerisationsreaktion beschleunigt, da der nucleophile Angriff der negativ geladenen Gruppen an die nicht-ionisierten Silanolspezies erleichtert wird. Die dabei entstehenden Oligomere tendieren zu Ringschlussreaktionen und es werden Partikel gebildet, die wiederum aufgrund ihrer hohen Ladungsdichte mit Monomeren reagieren, wodurch sie weiter an Größe gewinnen. Durch darauffolgende Schritte entstehen verzweigte, dichte, dreidimensionale Kieselsäurespezies. Es kann auch Depolymerisation stattfinden, da die Bildung von Siloxanbindungen eine reversible Reaktion darstellt. Aus diesem Grund dissoziieren kleinere Aggregate und die frei werdenden Monomere reagieren bevorzugt mit größeren Partikeln, wodurch deren Größe stetig zunimmt. In diesem Zustand spricht man von einem Sol beziehungsweise kolloidalem Silica.

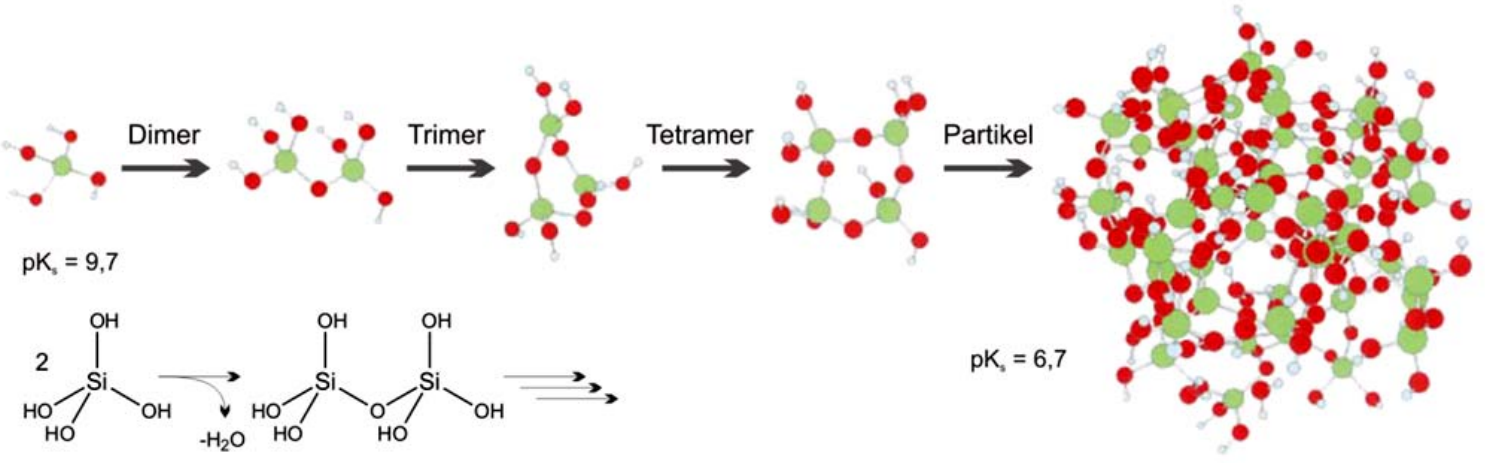

Abbildung 1.4 Kondensation von Kieselsäure und Bildung von Silica, entnommen von Coradin. ${ }^{39}$ 
Im alkalischen Milieu ist die Oberfläche der Aggregate negativ geladen, wodurch sie sich abstoßen und ein stabiles Sol entsteht, in dem die Partikel durch Ostwald-Reifung weiter wachsen. Bei neutralen bis sauren $\mathrm{pH}-$ Werten sind die Oberflächen der Partikel dahingegen ungeladen, was dazu führt, dass sich die Partikel aneinander anlagern. Sie verbinden sich $\mathrm{zu}$ verzweigten Ketten und bilden im Sol langsam dreidimensionale Netzwerke in Form eines Gels. In Anwesenheit von Salzen oder Flockungsmitteln wie kationischen Polymeren kommt es ausgehend vom Sol nicht zur Gelbildung, sondern es finden bevorzugt schnelle Präzipitationsreaktionen statt. Die hier beschriebenen Vorgänge sind in Abbildung 1.5 grafisch dargestellt.

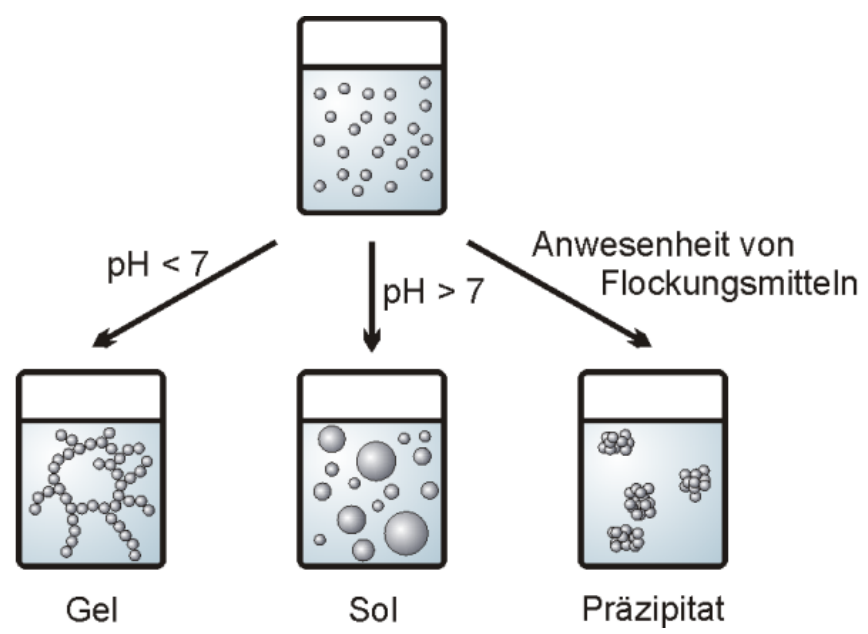

Abbildung 1.5 Unterschiede zwischen Bildung von Silica-Gel, -Sol und Präzipitat und deren Morphologie.

Der Unterschied zwischen diesen Aggregaten ist, dass in Gelen die Konzentration an Silicapartikeln genauso groß ist wie im umgebenden Sol, Dichte sowie Brechungsindex sind in diesem Fall gleich. In Präzipitaten jedoch ist die Konzentration an Silica höher als in der umgebenden Flüssigkeit, zugleich sind Dichte und Brechungsindex größer, weshalb Präzipitate sichtbar sind und abzentrifugiert werden können. Präzipitation findet statt, weil Flockungsmittel bei Zugabe zu einem Silicasol an $\mathrm{SiO}_{2}$-Partikel adsorbieren und diese vernetzen (Abbildung 1.6 A). Die Adsorption kann beispielsweise durch elektrostatische Attraktion zwischen kationischem Flockungsmittel und der negativ geladenen Silicaoberfläche stattfinden. Nur in Anwesenheit von ausreichend Flockungsmittel, also für den Fall, dass es ausreichend Kontaktstellen für die Silicapartikel gibt und somit dreidimensionale Netzwerke gebildet werden können, kommt es zur vollständigen Ausfällung. Sind zu wenige Polymere anwesend, adsorbieren diese zwar auf den Silicapartikeln, können diese jedoch nicht verbrücken; trotzdem kann ein Anteil an $\mathrm{SiO}_{2}$ präzipitieren. Ist die Konzentration dagegen zu hoch, können die Partikel komplett mit Polymer bedeckt 
werden und dadurch redispergieren. Auch die Ionenstärke spielt eine wichtige Rolle, da sie das Auf- beziehungsweise Entwickeln von Polymeren und damit das Bindungsverhalten bestimmt. Die Länge der Polymere beeinflusst ihre Fähigkeit Silicapartikel zu verbrücken, dementsprechend führt eine größere Länge dazu, dass geringere Polymerkonzentrationen für die Präzipitation benötigt werden. Ab einer Molekülmasse von $1000 \mathrm{~g} / \mathrm{mol}$ spielt dieser Effekt keine Rolle mehr.

Bei diesen Ausführungen wird immer von einer Adsorption der kationischen Polymere auf den Silicapartikeln ausgegangen. Dies wird beispielsweise durch das Auftreten von polyaminartigen Fasern auf der Oberfläche von Silica begründet. ${ }^{40}$ Dagegen wird in neueren Arbeiten zu Polyaminen und Silaffinen (siehe Kapitel 1.4) davon ausgegangen, dass diese Moleküle in wässrigen Medien phasenseparieren und dass dieser Prozess essenziell für die Silicabildung ist (Abbildung 1.6 C). Dabei spielt die Phosphorylierung von Silaffinen beziehungsweise die Anwesenheit multivalenter Anionen eine entscheidende Rolle, da die positiven Ladungen der basischen Moleküle eine Zusammenlagerung zu größeren Aggregaten verhindern. ${ }^{31,32,41}$ Laut diesen Hypothesen werden Kieselsäuremono- bis -oligomere auf Polymermikrotröpfchen adsorbiert beziehungsweise in ihnen gelöst, bevor die Kondensation zu Silica stattfindet, statt umgekehrt, wie oben beschrieben wurde.
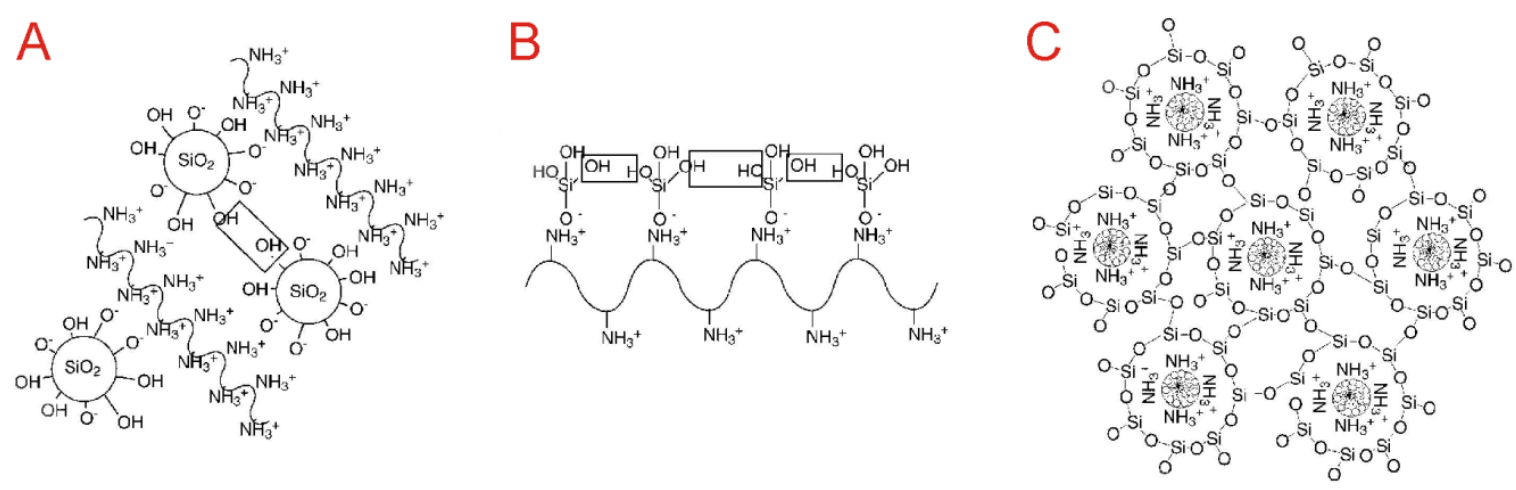

Abbildung 1.6 Der Einfluss von Proteinen auf die Silicabildung: A Positiv geladene Proteine verbrücken Silicapartikel und dirigieren ihre Aggregation. B Die Wechselwirkung von Kieselsäure mit den Ammoniumgruppen einer Proteinkette begünstigt die Kondensationsreaktion. C In einer phasenseparierten Suspension mit Mizellen amphiphiler kationischer Proteine fungieren diese als Template für die sich bildenden Silicastrukturen (entnommen von Coradin). ${ }^{39}$

Auf mechanistischer Ebene wurde in einigen Arbeiten vorgeschlagen, dass die Aminogruppen von Polyaminen den Übergangszustand der Kondensationsreaktion von Kieselsäure stabilisieren können, wenn sie günstig angeordnet sind (Abbildung 1.6 B). Die Polyamine fungieren demnach als Säure-Base-Katalysatoren und begünstigen den Protonentransfer bei den Kondensationsreaktionen. ${ }^{42-44}$ Die Rolle der Flockungsmittel geht 
diesen Arbeiten zufolge über reine Verbrückung von Silicapartikeln hinaus. Abbildung 1.6 (von Coradin und Lopez ${ }^{39}$ ) gibt die unterschiedlichen Einflüsse von positiv geladenen Flockungsmitteln auf die Silicapolymerisation wieder.

\subsection{Komponenten und Kontrolle der Silicabildung in Diatomeen}

Entscheidende Fortschritte zum Verständnis der Zellwandbildung der Diatomeen brachte die Charakterisierung von organischen Zellwandkomponenten. Bereits früh wurden in den Diatomeenzellwänden $\varepsilon$ - $N$-Trimethyl- $\delta$-hydroxylysin, sowohl in phosphorylierter als auch dephosphorylierter Form, sowie 3,4-Dihydroxyprolin entdeckt. ${ }^{45,46}$ Neben Polysacchariden wurden bis heute fünf Familien von Proteinen identifiziert: Frustuline, ${ }^{47,48}$ Pleuraline, ${ }^{34,49}$ Silaffine, ${ }^{31,50}$ die p150-Familie ${ }^{51,52}$ und jüngst die Silacidine ${ }^{53}$. Darüber hinaus wurden aus den Zellwänden langkettige Polyamine, die sogenannten long-chain polyamines (LCPA), isoliert. $^{54-56} \mathrm{Da}$ das Silaffin natSil-1A und auch LCPAs in vitro Silicananokugeln aus kieselsäurehaltigen Lösungen ausfällen, wird diesen Verbindungen eine besondere Rolle bei der Zellwandbiogenese zugeschrieben. ${ }^{57}$

Bei den Silaffinen (Abbildung 1.7 A) handelt es sich um Peptide, die überwiegend aus Serin, Lysin sowie Glycin aufgebaut sind und die eine hohe Zahl posttranslationaler Modifikationen aufweisen. So werden alle Serineinheiten der sill-Silaffine (natSil-1A1, natSil-1A2 und natSil-1B) von C. fusiformis phosphoryliert, während die $\varepsilon$-Aminogruppen der Lysineinheiten alkyliert werden. ${ }^{50}$ Bei den Alkylresten handelt es sich um $N$-methylierte Oligopropylenimineinheiten. Zusätzlich werden einige Lysingruppen am $\delta$-C-Atom hydroxyliert und phosphoryliert. ${ }^{31,58}$ Neben der sill-Gruppe wurden weitere Silaffine entdeckt, genauer natSil- $2^{59}$ in C. fusiformis sowie tpSil3 ${ }^{60,61}$ in T. pseudonana. All diese Silaffine weisen serin- und lysinreiche Domänen sowie ähnliche Modifikationen auf. Darunter fallen die Phosphorylierung von Hydroxyaminosäuren, $N$-Alkylierungen mit Methylgruppen sowie Polyaminresten und die Hydroxylierung von Lysin- und Prolinsubstituenten. Glykosylierung und Sulfatierung tritt dahingegen nur in einigen Silaffinen auf. $^{62}$

Die LCPAs (Abbildung 1.7 B) besitzen jeweils eine Grundeinheit, entweder Propyldiamin, Spermidin oder Putrescin, an die lineare Propylenimineinheiten geknüpft sind, deren Länge und $N$-Methylierung variiert. Jede Diatomeenspezies besitzt einen definierten Satz aus 
unterschiedlichen LCPAs. ${ }^{63}$ Die Kieselalgen Coscinodiscus asteromphalus, C. granii sowie Coscinodiscus wailesii weisen sogar ausschließlich Polyamine auf, bei ihnen konnten keine Silaffine identifiziert werden.
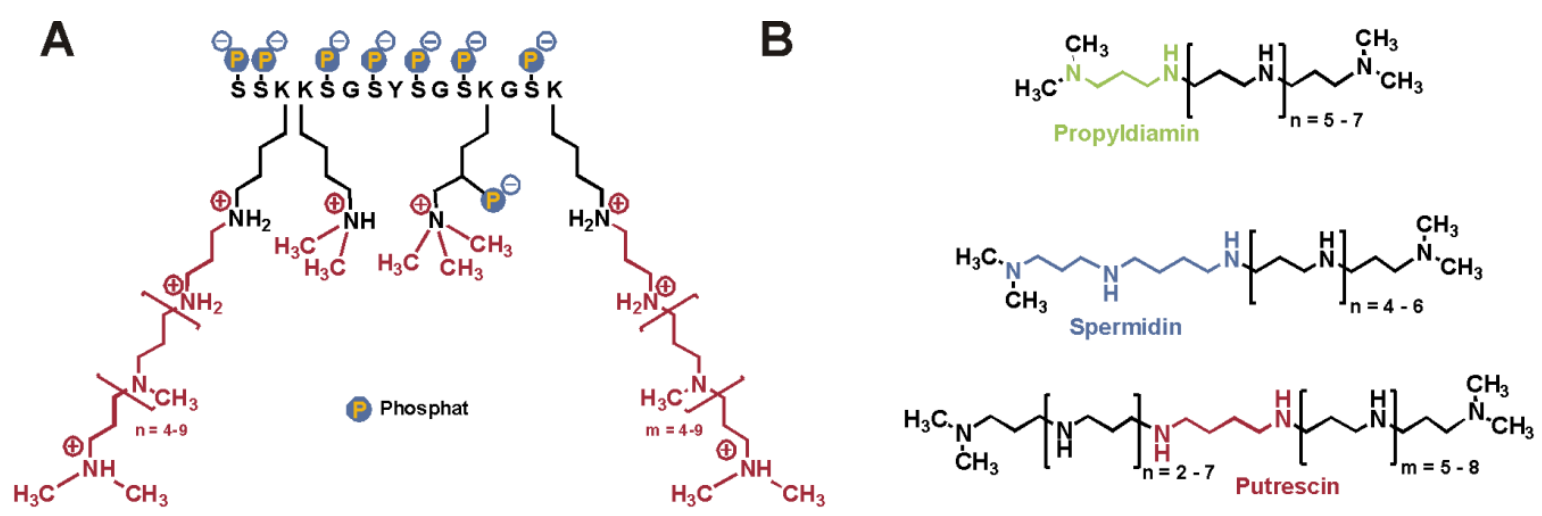

Abbildung 1.7 Strukturformeln von A natSil-1 $\mathrm{A}_{1}$ mit Ladungsverteilung bei $\mathrm{pH} \sim 5$ sowie B LCPAs. ${ }^{3,31}$

Nachdem die Silica-präzipitierende Funktion der Silaffine und LCPAs in vitro entdeckt wurde, konzentrierten sich die Untersuchungen auf den Einfluss von verschiedenen Reaktionsbedingungen auf Quantität, Kinetik und Struktur des gebildeten Silica. Zu diesem Zweck wurden Silaffine, LCPAs sowie synthetische Polyamine eingesetzt.

In diesem Zuge wurde geklärt, dass die Reaktion mit den Polyaminen ausschließlich in Anwesenheit multivalenter Anionen wie Phosphat stattfindet. Dies wird darauf zurückgeführt, dass eine Phasentrennung, durch die eine Mikroemulsion von Polyamintröpfchen in der wässrigen Phase entsteht, unabdingbar für die Präzipitation von Kieselsäure ist. Es wird angenommen, dass die hohe Anzahl positiver Ladungen in den Polyaminen Repulsion verursacht, die eine Akkumulation der Moleküle verhindert. Phosphat verbrückt die Moleküle und es kommt zur Bildung mikroskopischer Tröpfchen.

Diese These bestätigt sich bei natSil-1A, das eine zwitterionische Struktur aufweist. Liegt es in seiner natürlichen phosphorylierten Form (Abbildung 1.7 A) vor, müssen keine multivalenten Anionen zugegen sein. Wird das Molekül jedoch dephosphoryliert (Silaffin-1A), verliert es ebenfalls seine Präzipitations-induzierende Funktion, sofern nicht multivalente Anionen zugegeben werden. ${ }^{31}$ Die Aggregation der Moleküle, die zu einer lokalen Anreicherung basischer Substituenten führt, ist also neben der schon früher erkannten katalytischen Rolle der Aminoeinheiten ${ }^{42,64}$ ein entscheidender Faktor.

Im Zusammenspiel mit weiteren Faktoren wie Anionenkonzentration oder Anwesenheit anderer $\mathrm{SiO}_{2}$-präzipitierender Substanzen steigt die gebildete Silicamasse mit der Konzentration von Silaffinen. ${ }^{59,60}$ Die Präzipitationsreaktionen laufen allgemein auf einer 
schnellen Zeitskala ab. Kinetische Messungen zeigten, dass zehn Minuten ausreichen, um das Silica vollständig auszubilden. ${ }^{31,65}$

Im Gegensatz zu natSil-1A sind die anderen Silaffine, wie auch die Silacidine, saurer Natur. Sie selbst bewirken keine Silicapräzipitation, es wird ihnen jedoch eine dirigierende und kontrollierende Rolle zugesprochen. ${ }^{53,59,60}$ Sie fungieren in Mischungen mit Polyaminen anstelle von zum Beispiel Phosphat als Anionen, die die Phasenseparation bewirken, wobei das Zusammenspiel sowohl in Bezug auf Silicamenge als auch auf die Silicastruktur komplexer als mit anorganischen Anionen zu sein scheint. So kann ein zu hoher Anteil an sauren Verbindungen die Reaktion inhibieren, was mit Phosphat nicht beobachtet wurde. Basierend auf den vorgestellten Erkenntnissen wurden verschiedene Modelle entwickelt, die versuchen, die Bildung der filigranen Feinstrukturen der Diatomeenzellwände und das komplexe Zusammenspiel der beteiligten Komponenten zu erklären. Von den bereits in Abschnitt 1.2 angesprochenen Hypothesen werden die von Sumper und Kröger näher betrachtet. Sumper ${ }^{32}$ postuliert mehrere aufeinanderfolgende Phasentrennungsschritte, durch die Mikro- und Nanotröpfchen von LCPAs in wässriger Umgebung entstehen (Abbildung 1.8). Diese ordnen sich dicht gepackt in hexagonalen Strukturen im flachen SDV an. An den jeweiligen Grenzflächen der Tröpfchen findet die Polymerisation zu $\mathrm{SiO}_{2}$ statt, wodurch ein honigwabenähnliches Gerüst entsteht $(\mathrm{A} / \mathrm{E})$.

A
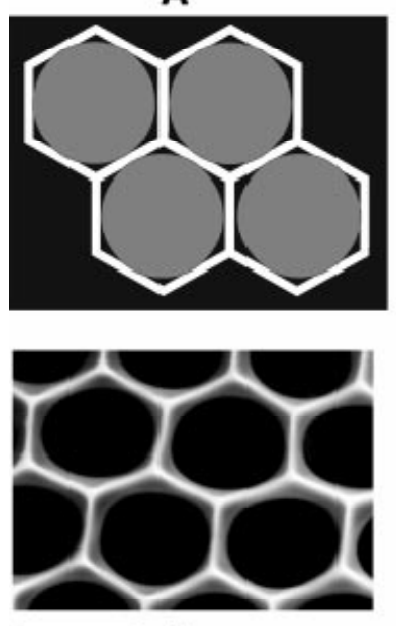

$2 \mu \mathrm{m}$
B
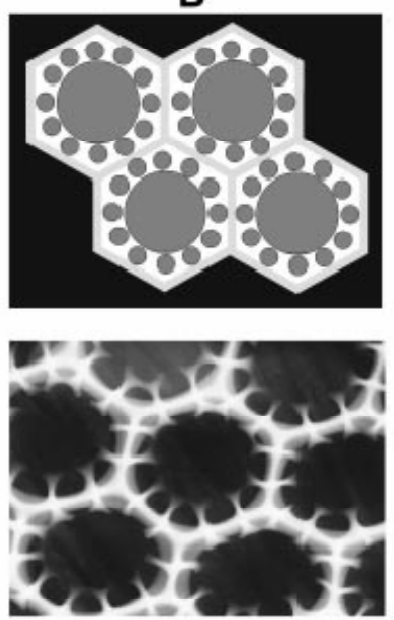

$\mathbf{F}$
C
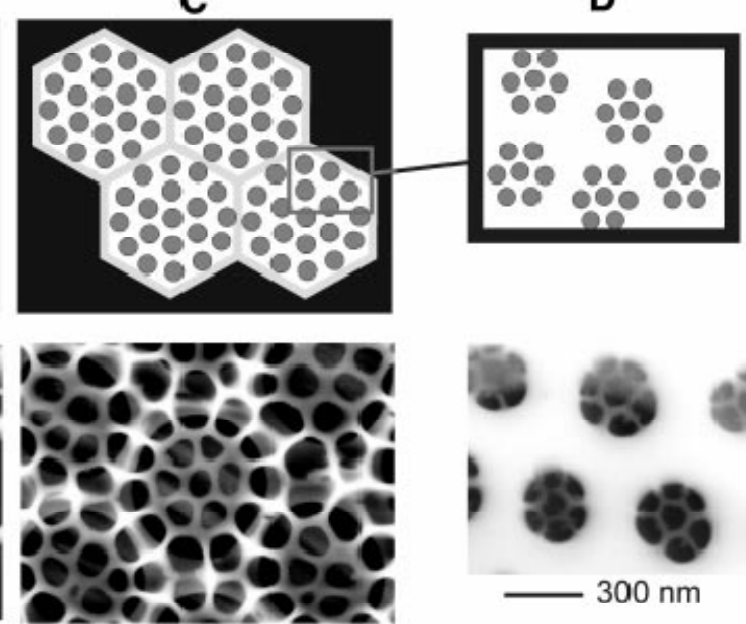

G

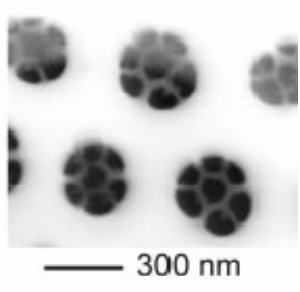

H

Abbildung 1.8 A-D Phasenseparationsmodell von Sumper sowie E-H SEM-Aufnahmen, die die Bildung der Silicazellwände von $C$. wailesii dokumentieren. ${ }^{32}$

Durch die Co-Präzipitation von Polyaminen und $\mathrm{SiO}_{2}$ wird ein gewisser Anteil der Polyamine verbraucht, was zu einer Verkleinerung der Tröpfchen führt. Sie zerfallen bei einer kritischen Größe in kleinere Aggregate (B/F). An den neu gebildeten Grenzflächen 
zwischen wässriger Phase und Polyamintröpfchen wiederholen sich die beschriebenen Vorgänge, was letztendlich in der Bildung der hierarchischen Strukturen der Zellwand resultiert $(\mathrm{C} / \mathrm{D} / \mathrm{G} / \mathrm{H})$.

Kröger $^{66}$ erweitert dieses Modell, indem er zusätzlich zu den LCPAs die Rolle der sauren Silaffine erklärt (Abbildung 1.9). Durch Interaktion dieser Komponenten entsteht eine phasenseparierte organische Matrix, deren Nanostruktur in großem Maße von den Silaffinen bestimmt wird. Durch lokale Konzentration von Polyaminen findet in diesen Bereichen die Silicabildung statt, während sie in silaffinreichen Bereichen inhibiert wird. Auf diesem Weg entsteht eine fein definierte Silicastruktur, die in verschiedenen Diatomeenspezies aufgrund unterschiedlicher Zusammensetzung von Silaffinen und auch Polyaminen spezifisch ist.
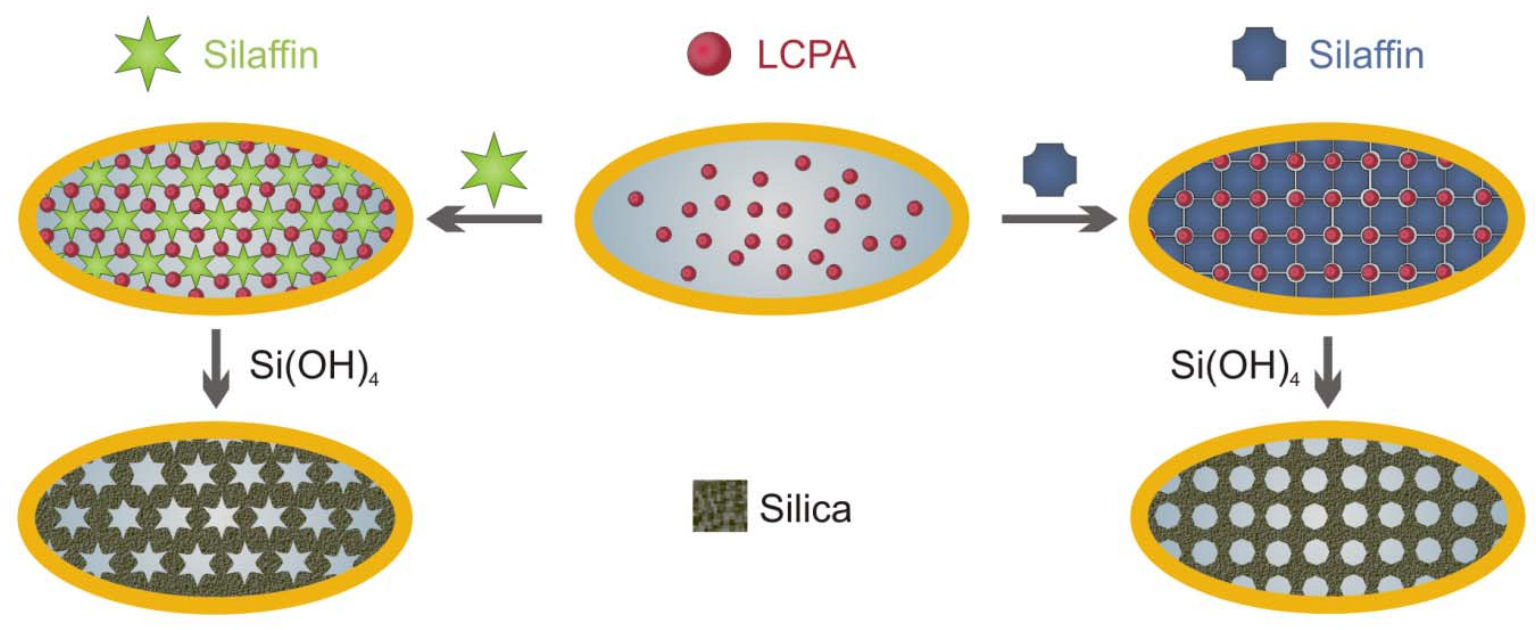

Abbildung 1.9 Die Silaffin-Matrix-Hypothese nach Kröger. ${ }^{66}$ Durch die Interaktion von Silaffinen mit LCPAs wird eine Phasenseparation induziert. Die entstehende Matrix beschleunigt und dirigiert die anschließende Silicabildung.

Es ist davon auszugehen, dass sowohl die Silaffine als auch die Polyamine sowie deren Aggregationsverhalten eine entscheidende Rolle spielen, indem sie sowohl die Polymerisation der Kieselsäure katalysieren als auch die Bildung hierarchischer Strukturen dirigieren. $\mathrm{Ob}$ und wie weitere Komponenten an diesen Prozessen beteiligt sind, ist noch nicht geklärt, genauso wenig, wie die komplexen Vorgänge genau vonstatten gehen. Den molekularen Mechanismus zu verstehen, mit dem eine einzelne Zelle das morphogenetische Programm für die Bildung von anorganischem Material ausführt, ist nicht nur ein faszinierendes biologisches Problem, sondern auch für die Nanomaterialforschung und Technologie interessant. ${ }^{67,68}$ Wie übersetzt eine Zelle DNA-Informationen in gemusterte dreidimensionale Strukturen? 



\section{Zielsetzung}

Die in Diatomeen stattfindenden Biomineralisationsprozesse, die zur Bildung ihrer Silicazellwände führen, sind bisher nur ansatzweise verstanden. Es wurden einige biologische Komponenten - wie beispielsweise Polyamine - identifiziert, die an diesen Prozessen beteiligt sind. Darüber hinaus existieren einige Modelle bezüglich ihrer Wirkungsweisen, wobei die genauen Zusammenhänge bislang nicht geklärt sind. Neben dem grundsätzlichen Interesse am Verständnis der molekularen Prozesse wird erhofft, das erworbene Wissen für die Bildung neuer Materialien nutzen zu können. So würden schnell verlaufende biomimetische Synthesen, die unter den gleichen milden physiologischen Bedingungen stattfinden, wie sie in Diatomeen vorherrschen und dabei eine ähnliche Nano- und Mikrostrukturierung mit hierarchischem Aufbau erzielen, einen großen Fortschritt gegenüber bisher entwickelten Silicasynthesen darstellen.

Im Rahmen dieser Arbeit sollten verschiedene Aspekte der Biomineralisation von Silica untersucht werden. Erstmals standen langkettige Polyamine mit definierten Strukturvariationen zur Verfügung, deren Einfluss auf die Silicapräzipitation geklärt werden sollte. Dabei galt es, Erkenntnisse sowohl über die quantitativen Auswirkungen mittels Siliciumkonzentrationsbestimmungen als auch über die entstehenden Strukturen anhand von elektronenmikroskopischen Messungen zu gewinnen. Ein weiteres Ziel dieser Arbeit war, die Bedeutung multivalenter Anionen auf die polyamininduzierte Silicabildung systematisch zu prüfen.

Im Fokus stand außerdem die Etablierung von Templatsystemen für biomimetische Silicapräzipitationen. Grundlegend dafür war die Suche nach geeigneten Verbindungen mit Silica-präzipitierenden Eigenschaften. Diese sollten amphiphil und oberflächenaktiv sein sowie über Molekülgeometrien verfügen, die die Bildung von selbstorganisierenden Templaten in wässriger Umgebung erlauben. Davon ausgehend sollten verschiedene Aggregate präpariert und charakterisiert werden, um diese im Anschluss für Silicapräzipitationen einzusetzen. Von Interesse war auch hier, entstandene Silicastrukturen quantitativ und morphologisch zu untersuchen, wofür geeignete Analysemethoden zu finden und anzuwenden waren. Die Etablierung eines Grundtemplatsystems bietet so die Möglichkeit, dieses zu variieren und zu erweitern, um sowohl Rückschlüsse auf die in Diatomeen ablaufenden Mechanismen ziehen zu können als auch Silica mit definierten Strukturen biomimetisch herstellen zu können. 



\section{Methoden und Materialien}

\subsection{Verwendete Substanzen}

\subsubsection{Polyamine}

Für die Erforschung des in vitro-Präzipitationsverhaltens langkettiger Polyamine in Lösung wurden sieben verschiedene Moleküle eingesetzt, die von R. Wieneke ${ }^{69}$ am Arbeitskreis von Prof. A. Geyer (Philipps-Universität Marburg, Fachbereich Chemie) synthetisiert wurden. Ihre Strukturformeln sind in Abbildung 3.1 dargestellt. Sie werden im Folgenden mit Abkürzungen benannt, die auf ihren Aufbau verweisen. Dabei steht C2 für Ethylgruppen, über die die Aminoeinheiten verbunden sind, entsprechend bezeichnet $\mathrm{C} 3$ Propyl- und C4 Butylgruppen. Beispielsweise besagt N7, dass ein Molekül insgesamt sieben Aminogruppen aufweist. Daraus leiten sich die Bezeichnungen für A) Pentapropylhexamin: C3N6, B) Undecapropyldodecamin: C3N12, C) Pentadecapropyloctadecamin: C3N18, D) Hexaethylheptamin: C2N7, E) Hexapropylheptamin: C3N7 sowie F) Hexabutylheptamin: C4N7 ab. Im Fall von G: C3N12Me verweist Me darauf, dass alle nicht endständigen Aminogruppen methyliert sind. Weitere Informationen zu den Verbindungen können Tabellen 4.1 und 4.2 sowie Abbildung 4.27 entnommen werden.

A<smiles>CC(N)CCNCCCN</smiles><smiles>CC(N)CCNCCN</smiles>

B<smiles>CCCCNCCCN</smiles>

E<smiles>CC(N)CCCNCCCN</smiles><smiles>CN(N)CCCN(C)CCCN</smiles>

C<smiles>CC(N)CCCNCCCN</smiles>

F<smiles>CC(C)(N)CCCCNC(C)(C)N</smiles>

Abbildung 3.1 Strukturformeln der langkettigen Polyamine A C3N6, B C3N12, C C3N18, D C2N7, E C3N7, F C4N7 und G C3N12Me.

Die Polyamine lagen alle als Feststoffe mit Trifluoressigsäure als Gegenionen vor. Beim Lösen sowohl in Wasser als auch in den verwendeten Phosphat- beziehungsweise Acetatpuffern entstanden klare Lösungen. Es wurden zunächst Stammlösungen in Reinstwasser hergestellt, in denen die jeweilige Konzentration an Aminogruppen $c_{\mathrm{N}}=10 \mathrm{mM}$ betrug. Etwa dreißig Minuten vor der Präzipitationsreaktion wurde ein definierter Volumenanteil einer Stammlösung mit Puffer verdünnt, so dass eine auf die Aminogruppen bezogene Konzentration von $c_{\mathrm{N}}=1 \mathrm{mM}$ resultierte. Das überwiegend 
verwendete Puffersystem bestand aus 30 mM Phosphatpuffer pH 6,8. Für die Untersuchung des Einflusses der Phosphationen wurde $30 \mathrm{~mm}$ Acetatpuffer $\mathrm{pH}$ 6,8 eingesetzt, bei dem entsprechend gewünschte Phosphatkonzentrationen durch Zugabe definierter Volumenanteile eines $500 \mathrm{~mm}$ Phosphatpuffer $\mathrm{pH}$ 6,8 eingestellt wurden.

Die Ermittlung der Säure-Base-Eigenschaften der Polyamine (Tabelle 4.2) wurde mit dem $\mathrm{pK}_{\mathrm{s}}$-Tool der Anwendung SPARC ${ }^{70}$ durchgeführt. Die genaue Vorgehensweise wird im Anhang 9.1 dargelegt.

\subsubsection{Aminolipide}

Neben den Biomineralisationsexperimenten mit Polyaminen wurden Versuche mit fünf verschiedenen amphiphilen Substanzen durchgeführt, die von R. Wieneke ${ }^{69,71}$ am Arbeitskreis von Prof. A. Geyer (Philipps-Universität Marburg, Fachbereich Chemie) synthetisiert und charakterisiert wurden. Ihr Aufbau orientiert sich insofern an Polyaminen beziehungsweise Silaffinen, als dass sie wie diese verschiedene Aminogruppen besitzen. Daneben enthalten sie Strukturelemente von Lipiden, genauer zwei hydrophobe Alkylketten. Diese sind mit den hydrophilen Aminokopfgruppen jeweils über ein Glycerinmolekül verbunden. Aufgrund dieser Eigenschaften werden die Verbindungen als Aminolipide bezeichnet. Die Zusätze in den Bezeichnungen in Form von primäres (pAL), sekundäres (sAL), tertiäres (tAL) beziehungsweise quartäres (qAL) Aminolipid, beziehen sich auf den Grad der $N$-Methylierung der jeweiligen Aminogruppe. Die Benennung Lysinollipid leitet sich von der Kopfgruppe der Substanz, dem Alkohol der Aminosäure Lysin, ab. Alle Strukturformeln sind in Abbildung 3.2 dargestellt. Weitere Informationen $\mathrm{zu}$ den Molekülen finden sich in Tabellen 5.1, 5.2, Abbildung 5.6 sowie in mehreren Diplomarbeiten ${ }^{72-74}$ wieder.

A

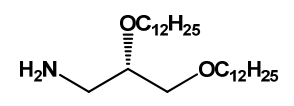

B

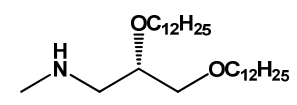

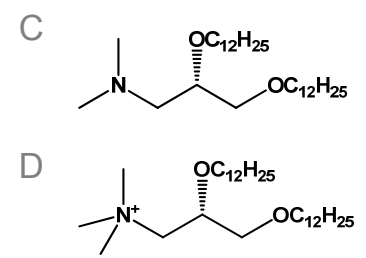

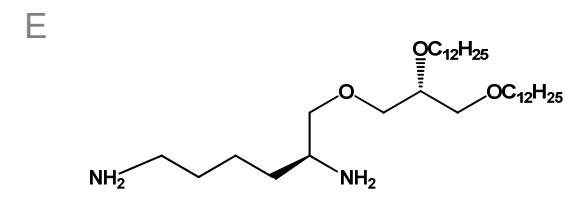

Abbildung 3.2 Strukturformeln der Aminolipide: A primäres Aminolipid, B sekundäres Aminolipid, C tertiäres Aminolipid, D quartäres Aminolipid sowie E Lysinollipid.

Die Verbindungen lagen in Form von Feststoffen vor. Da Verbindungen A-D hygroskopisch sind, wurden sie unter einer Argonatmosphäre bei $T=-18^{\circ} \mathrm{C}$ 
beziehungsweise in einem Exsikkator unter Vakuum über dem Trocknungsmittel Phosphorpentoxid gelagert. Zur Überprüfung ihrer Stabilität wurden sie massenspektrometrisch untersucht. ${ }^{74}$ Verbindung E wurde ohne weitere Vorkehrungen bei $T=-18^{\circ} \mathrm{C}$ aufbewahrt. Für die Verwendung wurden die Aminolipide in Chloroform gelöst, wobei die jeweilige Konzentration $(1,0-5,0) \mathrm{mg} / \mathrm{mL}$ betrug. Die genaue Konzentration von Verbindung E in Chloroform, die für die Filmwaagemessungen bekannt sein musste, wurde mittels quantitativer NMR- beziehungsweise Filmwaagemessungen $\left(A_{30}=(52,5 \pm 0,2) \AA^{2}\right)$ ermittelt. $^{72,73}$

Die Hauptphasenumwandlungstemperaturen $\left(T_{m}\right)$ der Aminolipide sind unbekannt, jedoch zeigte sich bei der Präparation von Lipidfilmen beziehungsweise Vesikelsuspensionen, dass außer dem primären Aminolipid alle Verbindungen bei Raumtemperatur lamellare Strukturen bilden. Daraus kann gefolgert werden, dass ihre Umwandlungstemperaturen $T_{m}<20^{\circ} \mathrm{C}$ sind. Für das primäre Aminolipid trifft das nicht zu, Lipidfilme und Vesikel mussten bei erhöhter Temperatur hergestellt werden $\left(T=60^{\circ} \mathrm{C}\right)$. Daraus wird für die Umwandlungstemperatur $20^{\circ} \mathrm{C}<T_{m}<60^{\circ} \mathrm{C}$ abgeleitet.

Die $\mathrm{pK}_{\mathrm{s}}$-Werte der Aminolipide wurden mit der Anwendung SPARC bestimmt. ${ }^{70}$ Genauere Informationen hierzu finden sich in Abschnitt 9.1 des Anhangs.

\subsubsection{C16-Glycerol- $\beta$-Lactosid (C16-GL)}

3-O- $\beta$-Lactosyl-1,2-di-O-hexadecyl-sn-glycerol, kurz C16-Glycerol- $\beta$-Lactosid (C16-GL) $\left(\mathrm{C}_{39} \mathrm{H}_{95} \mathrm{O}_{13}, M=865 \mathrm{~g} / \mathrm{mol}\right)$, ist ein synthetisches Glykolipid. Es besitzt ein Glyceringrundgerüst, an dessen $\mathrm{C} 1$ - und $\mathrm{C} 2$-Atom jeweils über eine Etherbindung eine gesättigte Hexadecylkette geknüpft ist (Abbildung 3.3). Die Alkylketten bilden den hydrophoben Teil des Moleküls. Die hydrophile Kopfgruppe des C16-Glycerol- $\beta$-Lactosids wird durch Lactose gebildet, ein aus einer Galactose- und einer Glucoseeinheit ( $\beta$-D-GalactopyranosylD-glucopyranosid) aufgebautes Disaccharid. Die Synthese von C16-GL wurde von R. Wieneke an der Philipps-Universität Marburg durchgeführt. ${ }^{69}$

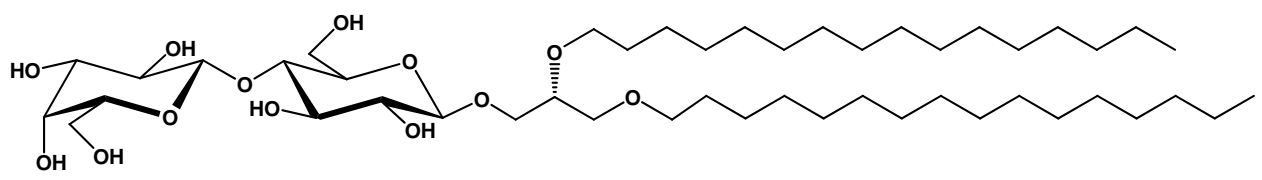

Abbildung 3.3 Strukturformel von C16-Glycerol- $\beta$-Lactosid. 
Die Verbindung lag als Feststoff vor und wurde bei $T=-18^{\circ} \mathrm{C}$ gelagert. Für anschließende Versuche wurde es in einer Konzentration von ca. $1 \mathrm{mg} / \mathrm{mL}$ mit einem Chloroform/ Methanol-Gemisch $(4 / 1, v / v)$ gemischt. Die Bestimmung der exakten Konzentrationen erfolgte mittels quantitativer NMR respektive Filmwaagemessungen $\left(A_{30}=(38,3 \pm 0,6) \AA^{2}\right){ }^{73}$ Da die Löslichkeit nicht ideal war und auch durch Variation des Mischungsverhältnisses der Lösungsmittel nicht verbessert werden konnte, wurden die Lösungen kurz vor Verwendung auf ca. $T=40^{\circ} \mathrm{C}$ erwärmt. Dadurch gingen zuvor sichtbare Feststoffflocken in Lösung. Filmwaagemessungen zeigten, dass die Langzeitstabilität von C16-GL in Lösungsmittel im Gegensatz zu den Aminolipiden gering war. Aus diesem Grund wurden die Lösungen maximal zwei Monate verwendet.

Die Phasenumwandlungstemperatur $T_{m}$ von C16-GL ist unbekannt, wird jedoch auf $T_{m} \approx 85^{\circ} \mathrm{C}$ geschätzt. Der Wert stammt aus einem Vergleich mit einem Glykolipid (C14GL), das Tetradecylketten aufweist, ansonsten jedoch identisch aufgebaut ist und eine Hauptphasenumwandlungstemperatur von $T_{m}=66{ }^{\circ} \mathrm{C}$ besitzt. ${ }^{75}$ Bei Phosphatidylcholinen bewirkt die Verlängerung der beiden Alkylketten von C14 auf C16 eine Erhöhung der Umwandlungstemperatur um ca. $20{ }^{\circ} \mathrm{C},{ }^{76}$ weshalb angenommen wird, dass $T_{m}$ von C16-GL gleichfalls etwa $20^{\circ} \mathrm{C}$ über der von C14-GL liegt.

\subsubsection{1,2-Dipalmitoyl-sn-glycero-3-phosphatidylcholin (DPPC)}

1,2-Dipalmitoyl-sn-glycero-3-phosphatidylcholin $\left(\mathrm{C}_{40} \mathrm{H}_{80} \mathrm{NO}_{8} \mathrm{P}, \quad M=734 \mathrm{~g} / \mathrm{mol}\right)$ ist ein Phospholipid mit amphiphiler Struktur, aufgebaut aus einem hydrophoben Teil mit Fettsäureresten sowie einer hydrophilen Kopfgruppe. Das Glycerinrückgrad ist an den Hydroxylgruppen von C1 und C2 jeweils mit einer Palmitinsäure verestert. Am Sauerstoff von C3 ist Phosphatidylcholin gebunden. Die Hauptphasenumwandlungstemperatur von DPPC liegt bei $T_{m}=41^{\circ} \mathrm{C} .^{77}$

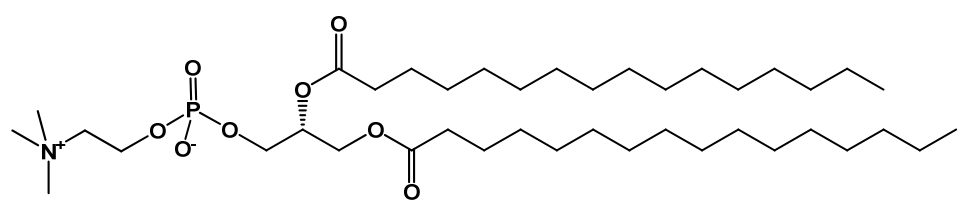

Abbildung 3.4 Strukturformel von DPPC. 


\subsubsection{Fluoreszenzsonden}

\section{Sulforhodamin 101 DHPE}

Bei Sulforhodamin 101 DHPE (in Form des Triethylammoniumsalzes, $\mathrm{C}_{74} \mathrm{H}_{117} \mathrm{~N}_{4} \mathrm{O}_{14} \mathrm{PS}_{2}$, $M=1381,84 \mathrm{~g} / \mathrm{mol}$ ) handelt es sich um ein Kopfgruppen-markiertes Glycerophospholipid (Abbildung 3.5). Der Fluorophor wird bei einer Wellenlänge von $\lambda=538 \mathrm{~nm}$ maximal angeregt und emittiert bei $\lambda=601 \mathrm{~nm}$. Außerdem ist bekannt, dass sich Sulforhodamin 101 DHPE bevorzugt in fluiden Membranbereichen anreichert. ${ }^{78}$ Die Substanz wurde in einer Konzentration von $0,69 \mathrm{mg} / \mathrm{mL}$ in einem Chlorform/Methanol(4/1, v/v)-Gemisch gelöst und bei $T=-18^{\circ} \mathrm{C}$ gelagert.

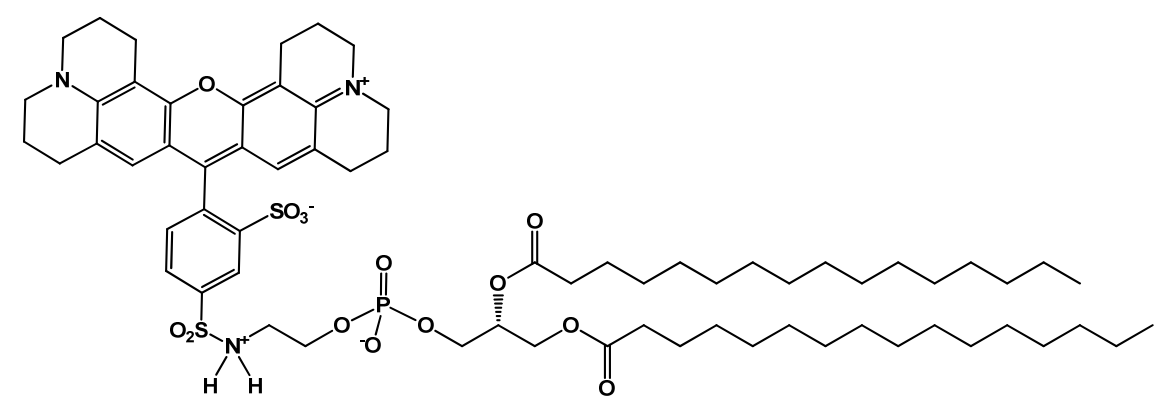

Abbildung 3.5 Strukturformel von Sulforhodamin 101 DHPE.

\section{$\beta$-BODIPY 500/510 $\mathrm{C}_{12}$-HPC}

Auch $\beta$-BODIPY 500/510 $\mathrm{C}_{12}$-HPC $\left(\mathrm{C}_{46} \mathrm{H}_{79} \mathrm{BF}_{2} \mathrm{~N}_{3} \mathrm{O}_{8} \mathrm{P}, M=881,93 \mathrm{~g} / \mathrm{mol}\right)$ verteilt sich in phasenseparierten Membranen nicht gleichmäßig und wird überwiegend in fluiden Bereichen angereichert. ${ }^{79,80}$ Allerdings ist in diesem Molekül der Fluorophor an das Ende einer der Fettsäureseitenketten des Phospholipids gebunden (Abbildung 3.6). Die maximale Absorption findet bei $\lambda=500 \mathrm{~nm}$ statt, die Emissionmaximum liegt bei $\lambda=510 \mathrm{~nm}$. Die als Feststoff vorliegende Verbindung wurde in Chloroform gelöst, wobei die Konzentration $0,2 \mathrm{mg} / \mathrm{mL}$ betrug, und bei $T=-18{ }^{\circ} \mathrm{C}$ aufbewahrt.

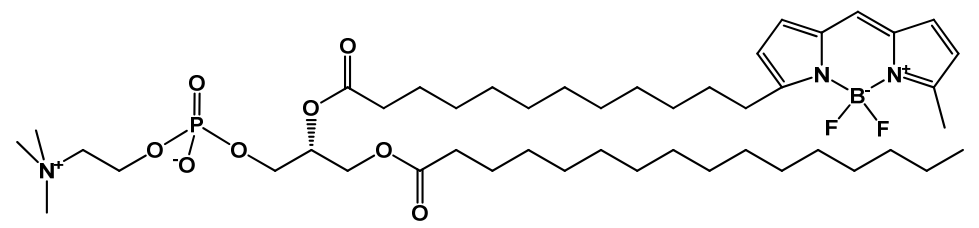

Abbildung 3.6 Strukturformel von $\beta$-BODIPY 500/510 $\mathrm{C}_{12}$-HPC. 


\subsection{Präparative Methoden}

\subsubsection{Vesikelpräparation}

Vesikel wurden zum einen für die Untersuchung der Präzipitationsreaktionen mit den Aminolipiden benötigt. Zum anderen dienten sie der Präparation festkörperunterstützter planarer Doppelschichten des Lysinollipids, die für anschließende Silicabildungsexperimente eingesetzt wurden. Es wurden große unilamellare Vesikel (large unilamellar vesicles, LUV) verwendet, deren Herstellung nach der Extrusionsmethode ${ }^{81}$ erfolgte. Sowohl für die Bildung der Vesikel als auch für die später erläuterten Spreitprozesse ist es nötig, dass sich die Lipide in der fluiden Phase befinden. Aus diesem Grund muss bei allen Präparationsschritten über der jeweiligen Hauptphasenumwandlungstemperatur $T_{m}$ der Lipide gearbeitet werden. Wie in Abschnitt 3.1.2 dargelegt, war für fast alle Aminolipide Raumtemperatur ausreichend, lediglich für das primäre Aminolipid musste eine erhöhte Temperatur von ca. $60^{\circ} \mathrm{C}$ gewählt werden.

\section{Herstellung von Lipidfilmen}

Zunächst wurde ein definiertes Volumen der jeweiligen Stammlösung des Aminolipids in ein Reagenzglas pipettiert. Nach Bedarf wurde ein entsprechender Anteil Fluorophorlösung hinzupipettiert. Das Lösungsmittel wurde durch Anlegen eines schwachen Stickstoffstroms aus der Probe, die sich im Wasserbad bei $T \approx 30^{\circ} \mathrm{C}$ befand, entfernt, wodurch sich am Boden des Reagenzglases Lipidfilme ausbildeten. Im Fall des primären Aminolipids betrug die Wasserbadtemperatur $60^{\circ} \mathrm{C}$ und es wurde kein Stickstoffstrom verwendet, sondern solange gewartet, bis das Lösungsmittel vollständig verdunstet war. Im Anschluss wurden die Filme bei entsprechender Temperatur $\left(30^{\circ} \mathrm{C}\right.$ oder $60^{\circ} \mathrm{C}$ ) für drei Stunden im Vakuum gelagert, um Lösungsmittelreste zu entziehen. Die Lipidfilme wurden optisch dahin gehend geprüft, dass sich keine kristallinen Substanzanhäufungen gebildet hatten. War dies der Fall, wurden die Filme erneut in Chloroform aufgenommen und die beschriebene Prozedur wiederholt, bis eine gleichmäßige Filmbildung eintrat. Die Lagerung der Lipidfilme erfolgte bei $T=4{ }^{\circ} \mathrm{C}$. 


\section{Präparation von LUVs}

Zur Präparation von LUVs wurde ein Lipidfilm für fünfzehn Minuten bei entsprechender Temperatur in Puffer rehydratisiert und anschließend dreimal im Abstand von fünf Minuten für dreißig Sekunden im Vortexer geschüttelt. Die entstehende Suspension wurde in einem Miniextruder (LiposoFast, Avestin, Ottawa, Kanada) 31mal durch eine Polycarbonatmembran mit Poren eines definierten Durchmessers gedrückt. In allen Fällen wurden die Suspensionen bei diesem Vorgang klar, sofern sie das nicht schon zuvor waren. Die Entstehung von LUVs konnte mittels dynamischer Lichtstreuungsmessungen bestätigt werden (Kapitel 5.2). Porendurchmesser, Lipidkonzentrationen und Puffersysteme finden sich in den jeweiligen Abschnitten der Ergebnisse.

\subsubsection{Herstellung von Lipiddoppelschichten mittels Spreiten von Vesikeln}

Unter den richtigen Bedingungen adsorbieren unilamellare Vesikel an hydrophilen Substratoberflächen und bilden durch Spreiten in einem selbstorganisierenden Prozess Lipiddoppelschichten (Abbildung 3.7).
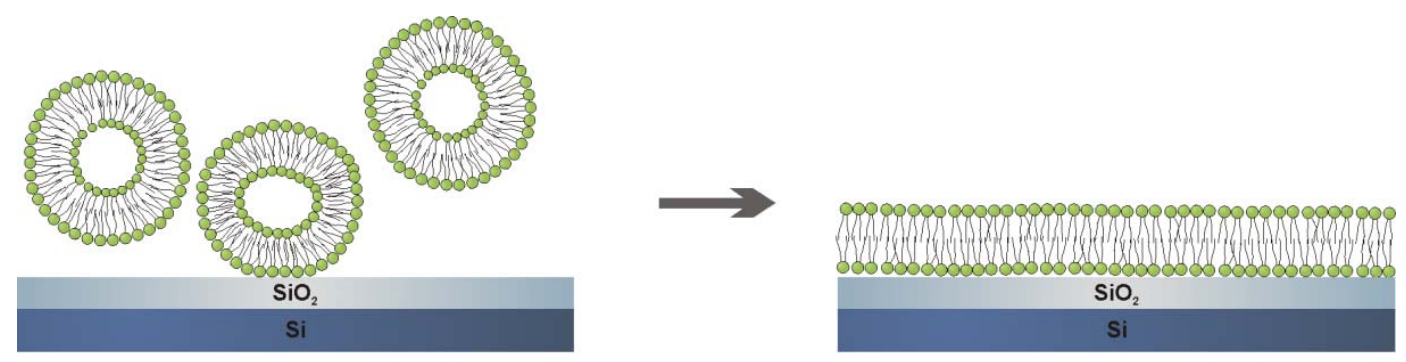

Abbildung 3.7 Schematische Darstellung des Spreitprozesses von Vesikeln auf einem hydrophilen Substrat, hier ein Siliciumwafer mit $\mathrm{SiO}_{2}$-Oberfläche.

Hierzu verwendete Lysinollipidvesikel wurden in $25 \mathrm{mM} \mathrm{MES} / 500 \mathrm{mM} \mathrm{KCl} \mathrm{pH} \mathrm{6,0}$ hergestellt. Als Substrate dienten Siliciumwafer oder Glimmer. Die Siliciumwafer wurden durch zwanzigminütige Inkubation in einer Lösung aus $\mathrm{H}_{2} \mathrm{O} / \mathrm{NH}_{3}\left(25 \%\right.$ ig) $/ \mathrm{H}_{2} \mathrm{O}_{2}(30 \%$ ig) $(5 / 1 / 1)(v / v / v)$ bei $T=70{ }^{\circ} \mathrm{C}$ vorbereitet, wodurch eine saubere hydrophile $\mathrm{SiO}_{2}-\mathrm{Oberfläche}$ gewährleistet wird. Im Anschluss wurden sie mehrmals mit Reinstwasser gespült und in selbem gelagert, bevor sie noch am gleichen Tag benutzt wurden. Welche Siliciumwafer verwendet wurden, ist im jeweiligen Ergebnisteil erwähnt. Glimmer wurde frisch mit Hilfe von Klebeband gespalten und ohne weitere Behandlung verwendet. Die Suspensionen der 
LUVs wurden bei Raumtemperatur entweder direkt auf das Substrat appliziert oder im Fall der Ellipsometriemessungen zu einer sich in der Messzelle befindenden Pufferlösung gegeben. In diese Messzelle war der Siliciumwafer zuvor eingebaut worden. Nach einstündiger Inkubation wurde die Probe mit Puffer oder Reinstwasser gespült, um überschüssige Substanz zu entfernen. Die Membranausbildung wurde ellipsometrisch verfolgt und fluoreszenz- sowie rasterkraftmikroskopisch überprüft (Kapitel 6.1.1).

\subsubsection{Herstellung von Lipiddoppelschichten mittels Langmuir- Blodgett-Technik}

Neben den durch das Spreiten von Vesikeln präparierten Membranen, kamen auch mittels Langmuir-Blodgett-Übertrag (Abbildung 3.8) hergestellte Membranen zum Einsatz. Im Gegensatz zum Spreiten von Vesikeln, können mit dieser Methode asymmetrische Doppelschichten erzeugt werden. Des Weiteren sind Parameter wie der Oberflächendruck und die Übertragsgeschwindigkeit, welche die Eigenschaften der immobilisierten Lipidfilme beeinflussen, variabel. Im Fall von binären phasenseparierten Monoschichten erlaubt die Methode weitestgehend die Fixierung der Filme in diesem Zustand.

\section{Hydrophiler Übertrag}

Bei dieser Methode werden Lipidmonoschichten auf einer Subphase, im Rahmen dieser Arbeit stets Reinstwasser, gespreitet. Hierzu wird die jeweilige Substanz (nach Bedarf mit einem Anteil Fluoreszenzmarker) in Lösungsmittel mit einer Mikrospritze vorsichtig auf die Wasseroberfläche aufgetragen. Das Lösungsmittel verdunstet innerhalb von fünfzehn Minuten. In einem Filmwaagetrog (Abbildung 3.16) wurde mit Hilfe von Barrieren der Oberflächendruck bis zu einem bestimmten Wert erhöht und während der gesamten Prozedur konstant gehalten. Zur Immobilisierung der ersten Monoschicht (DPPC) wird ein unter der Wasseroberfläche befindliches Substrat (Siliciumwafer oder Glimmer, siehe Abschnitt 3.2.2) mit einer Geschwindigkeit von $0,2 \mathrm{~mm} / \mathrm{min}$ (entspricht einer Geräteeinstellung von 7) durch die Grenzfläche nach oben gezogen. Durch attraktive Interaktionen der polaren Kopfgruppen mit der hydrophilen Substratoberfläche lagert sich der Film ab, wobei eine planare hydrophobe Oberfläche entsteht (Abbildung 3.8 A). Die beschichteten Substrate werden mindestens über Nacht in einem Exsikkator über Kieselgel (Orangegel) ohne angelegtes Vakuum getrocknet. 


\section{Hydrophober Übertrag}

Der oben beschriebene Vorgang wird wiederholt, mit dem Unterschied, dass nun das Substrat von oben durch die Grenzfläche gedrückt wird (Abbildung $3.8 \mathrm{~B}$ ), wobei auch hier die Geschwindigkeit $0,2 \mathrm{~mm} / \mathrm{min}$ betrug. Aufgrund von hydrophoben Wechselwirkungen zwischen den Alkylketten der amphiphilen Substanzen entsteht eine Doppelschicht, die bei Lagerung unter Wasser bis zu einen Tag stabil ist. Wird für anschließende Versuche ein Puffersystem über der Membran benötigt, wird das Wasser durch Spülen mit Puffer ersetzt, wobei darauf zu achten ist, dass die Oberfläche, um eine Zerstörung der Membran zu verhindern, niemals Kontakt zur Luft haben darf. Mittels Fluoreszenz- und Rasterkraftmikroskopie wurden die Membranen charakterisiert (Kapitel 6.1.1 und 6.2.3).
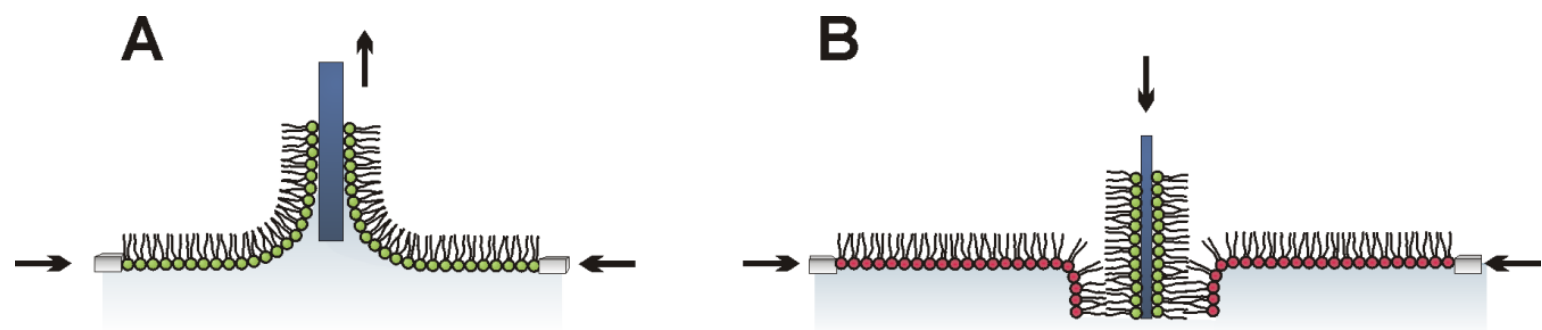

Abbildung 3.8 Schematische Darstellung der Immobilisierung von Lipidmonoschichten mittels LangmuirBlodgett-Technik. A Hydrophiler und B hydrophober Übertrag.

Die Oberflächendrücke finden sich bei den entsprechenden Ergebnissen. Die Barrierengeschwindigkeit betrug beim Übertrag der ersten Monoschicht (DPPC) $2,5 \mathrm{~cm}^{2} / \min$ (Geräteeinstellung 20), wohingegen sie beim zweiten, dem hydrophoben Übertrag (Lysinollipid, Lysinollipid/C16-GL) ca. 4,9 cm²/min (Geräteeinstellung 40) betrug. Sie wurde hier erhöht, um den Vorgang zu beschleunigen und somit zu hohe Substanzverluste von Lysinollipid in die Subphase über die Zeit zu vermeiden. Da während des gesamten Vorgangs der Oberflächendruck konstant gehalten wird, werden die Barrieren permanent automatisch nachgeregelt, damit Substanzverluste ausgeglichen werden. Um sicherzustellen, dass die Fläche des Filmwaagetrogs für die Prozedur ausreicht, wurde viel Substanz gespreitet, im Fall von DPPC bis zu einem Oberflächendruck von $\pi \approx 4 \mathrm{mN} / \mathrm{m}$ und im Fall von Lysinol beziehungsweise Lysinol/C16-GL bis zu $\pi \approx 7 \mathrm{mN} / \mathrm{m}$. 


\subsubsection{Silicapräzipitationsreaktionen}

Zur Herstellung von monomerer Kieselsäure $\mathrm{Si}(\mathrm{OH})_{4}$ wurde ein Alkoxysilan, genauer Tetramethoxysilan (TMOS, $\left.\mathrm{Si}\left(\mathrm{OCH}_{3}\right)_{4}\right)$, verwendet. Wird TMOS mit Salzsäure versetzt, setzt eine saure Hydrolyse ein, bei welcher unter Abspaltung von vier Äquivalenten Methanol $\mathrm{Si}(\mathrm{OH})_{4}$ entsteht (Abbildung 3.9). Im Detail wurde ein definiertes Volumen TMOS $(c=6,8 \mathrm{M})$ mit $1 \mathrm{mM} \mathrm{HCl}$ im Verhältnis 3/17 $(v / v)$ gemischt, kräftig geschüttelt bis eine klare Lösung vorlag, und für zwanzig Minuten inkubiert. Die Konzentration der resultierenden Kieselsäure betrug in allen Fällen $c=1 \mathrm{mM}$. Es wurde darauf geachtet, dass die Lösung sofort weiterverwendet wurde, um etwaige Polymerisationsprozesse zu verhindern.

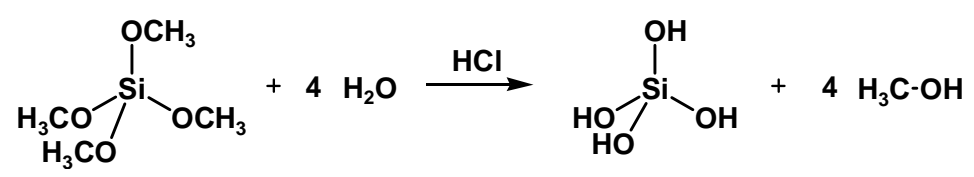

Abbildung 3.9 Reaktionsgleichung der sauren Hydrolyse von Tetramethoxysilan.

Bei Präzipitationstests mit Polyaminen (Kapitel 4.2.1) stellte sich heraus, dass eine Variation der Konzentration von $\mathrm{Si}(\mathrm{OH})_{4}$ im Bereich von $c=(40-100) \mathrm{mM}$ keinen Einfluss auf die entstehenden Produkte hat. Es wird angenommen, dass in beiden Fällen die monomere Kieselsäure im Überschuss vorliegt. Es wurde bei allen Experimenten, soweit nicht anders erwähnt, eine Konzentration von $c=100 \mathrm{mM}$ eingesetzt.

\section{Silicabildung mit Polyaminen und Aminolipidvesikeln}

Im Fall der Polyamine und der vesikulären Aminolipidsysteme wurde für die Silicabildungsexperimente die jeweilige Suspension in einem Eppendorfgefäß mit der hydrolysierten Kieselsäure versetzt, die Probe im Anschluss für dreißig Sekunden geschüttelt und, falls nicht anders beschrieben, für zehn Minuten inkubiert. Im Anschluss wurde die Reaktion gestoppt, indem das entstandene Silica bei $14000 \times g$ für fünf Minuten abzentrifugiert wurde. Die überstehende Flüssigkeit wurde abgenommen und die Probe mit Reinstwasser versetzt, geschüttelt und erneut zentrifugiert. Dieser Schritt wurde dreimal wiederholt um überschüssige Kieselsäure $\mathrm{zu}$ entfernen. Die so entstandenen Proben wurden entweder elektronenmikroskopisch untersucht oder die enthaltene Silicamenge mittels der $\beta$-Molybdatmethode detektiert (Abschnitte 4 sowie 5.3). 


\section{$\mathrm{SiO}_{2}$-Präzipitation an planaren Membranen}

Für Präzipitationsreaktionen an planaren Membransystemen wurden die mit Puffer überschichteten Proben in Teflontrögen beziehungsweise einer Ellipsometriemesszelle platziert. Die Kieselsäure wurde zu der Pufferlösung pipettiert und in den Teflontrögen durch wiederholtes Aufziehen und Ablassen von Flüssigkeit mit der Pipette durchmischt. In der Ellipsometriezelle wurde die Durchmischung während der gesamten Reaktionszeit durch Rühren mit einem Magnetrührstäbchen gewährleistet. Nach der jeweiligen Inkubationszeit (siehe Kapitel 6.1.2 und 6.2.4) wurden die Proben mit Reinstwasser gespült, um danach mittels Fluoreszenz- und Rasterkraftmikroskopie untersucht zu werden. Ein Teil der Proben wurde getrocknet, indem das Wasser vorsichtig mit einer Pipette abgezogen wurde. Vor darauffolgenden AFM- oder SEM-Messungen wurde mindestens ein Tag gewartet, um zu gewährleisten, dass das Wasser vollständig verdunsten konnte.

\subsection{Messmethoden}

\subsection{1 $\beta$-Molybdatmethode}

Um die Menge an Silica, die bei Präzipitationsreaktionen mit Polyaminen und Aminolipidvesikeln entsteht, quantitativ ermitteln zu können, wurde ein modifizierter $\beta$-Molybdattest angewendet. $^{35,82}$ Er beruht auf der Bildung eines gelben $\beta$-Silicomolybdänsäure-Komplexes (Abbildung 3.10), dessen Konzentration photometrisch bestimmt werden kann.

$$
\mathrm{H}_{4} \mathrm{SiO}_{4}+12 \mathrm{H}_{2} \mathrm{MoO}_{4} \longrightarrow \mathrm{H}_{4} \mathrm{SiO}_{4} \cdot 12 \mathrm{MoO}_{3}+12 \mathrm{H}_{2} \mathrm{O}
$$

Abbildung 3.10 Reaktion von Kieselsäure mit Molybdat zum gelben $\beta$-Silicomolybdänsäure-Komplex.

Anders als beim Molybdänblautest wird das Produkt nicht reduziert. Der Hauptunterschied zwischen den beiden Methoden ist die Sensitivität, die bei der Molybdänblaumethode höher ist. Dementsprechend wählt man eine der beiden nach der zu detektierenden Kieselsäurekonzentration. Der $\beta$-Molybdattest ist sensitiv auf Kieselsäuremono- und -dimere. Aus diesem Grund muss Silica vor der Bestimmung hydrolysiert werden. 


\section{Durchführung des Nachweises}

Gewaschene Silicapellets wurden mit 2 M Natronlauge versetzt, geschüttelt und sechzig Minuten bei Raumtemperatur inkubiert, wobei sich der Feststoff auflöste. 1,35 mL Salzsäure (37\%) wurden mit 40,3 mL Reinstwasser gemischt. 774,2 mg $\left[\left(\mathrm{NH}_{4}\right)_{6} \mathrm{Mo}_{7} \mathrm{O}_{24} \cdot 4 \mathrm{H}_{2} \mathrm{O}\right]$ wurden in $9,7 \mathrm{~mL}$ Reinstwasser gelöst. Die beiden Lösungen wurden vereint und der $\mathrm{pH}-$ Wert wurde mit $2 \mathrm{M} \mathrm{NaOH}$ auf 1,12 eingestellt. Von der gelösten Silicaprobe wurden definierte Volumenanteile von (0, 20 oder 40) $\mu \mathrm{L}$ und ein entsprechendes Volumen Natronlauge (0, 20 oder 40) $\mu \mathrm{L}$ sowie jeweils $200 \mu \mathrm{L}$ Wasser und $800 \mu \mathrm{L}$ der Molybdatlösung in eine Plastikküvette $(d=1 \mathrm{~cm})$ pipettiert und gut durchmischt. Zur Erstellung einer Eichgeraden wurde eine Siliciumstandardlösung verwendet, von der jeweils ein definiertes Volumen wie auch die Proben mit Natronlauge, Wasser und Molybdatlösung versetzt wurde. Die Absorptionen wurden mittels eines UV/Vis-Spektrophotometers bei einer Wellenlänge von $\lambda=370 \mathrm{~nm}$ bestimmt. Über die Gleichung der Anpassungsgeraden der Eichmesswerte wurde die Siliciumkomzentration beziehungsweise -masse bestimmt.

Allgemein ist zu beachten, dass alle Lösungen frisch herstellt werden und die Benutzung von Glasgefäßen vermieden werden muss. Auch die zur pH-Wert-Einstellung verwendete Natronlauge wurde in einem Kunststoffbehältnis aufbewahrt.

\subsubsection{Elektronenmikroskopie}

Elektronenmikroskopische Methoden basieren auf den Interaktionen der Elektronen eines Teilchenstrahls mit der Materie einer Probe (Abbildung 3.11 A). Es wird zwischen zwei Typen der Wechselwirkung unterschieden, elastischer und inelastischer Streuung. ${ }^{83}$

Im Fall von elastischer Streuung (Abbildung 3.11 B) findet keine (oder eine vernachlässigbar kleine) Energieübertragung von den primären Elektronen (PE) auf die Probe statt. Die Eintrittsenergie der Elektronen entspricht der Austrittsenergie. Das einfallende Elektron durchtritt die Probe entweder ohne jegliche Wechselwirkung (direct beam) oder es wird aufgrund von Coulomb Interaktionen mit dem positiven Potenzial der Atomkerne gestreut, wobei auch eine vollständige Rückstreuung auftreten kann (backscattered electrons, BSE) $)^{84}$. Erstere Signale werden vor allem in der Transmissionselektronenmikroskopie (transmission electron microscopy, TEM) genutzt, die BSE jedoch werden für Rasterelektronenmikroskopie (scanning electron microscopy, SEM) genutzt. 
A

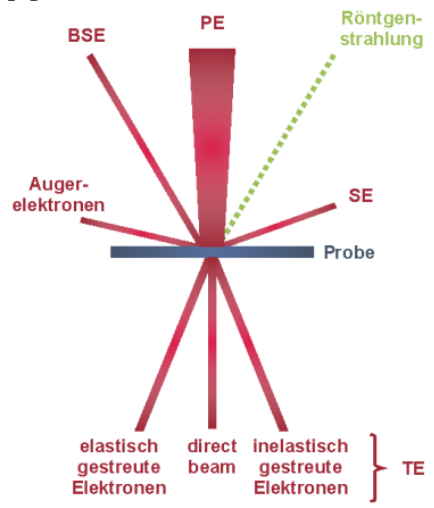

B

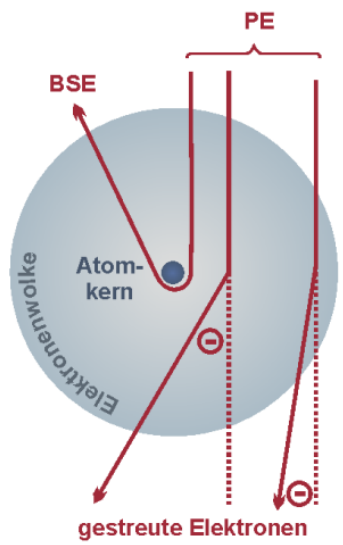

C

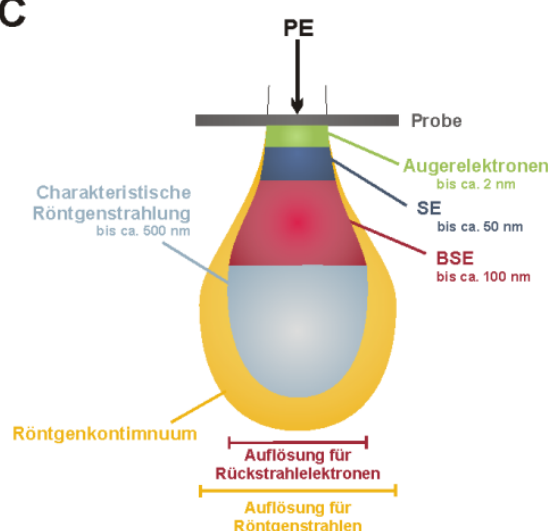

Abbildung 3.11 A Mögliche Elektronen-Materie-Interaktionen die beim Auftreffen eines Elektronenstrahls auf eine Probe auftreten. B Verschiedene Varianten der elastischen Streuung. C Diffusionszone der Primärelektronen mit Erzeugungsbereich der Wechselwirkungsprodukte. PE: Primärelektronen, SE: Sekundärelektronen, BSE: Rückstreuelektronen, TE: transmittierte Elektronen. A/B nach Krumeich ${ }^{84}$, C nach Raschke ${ }^{85}$.

Bei inelastischer Streuung kommt es zu Wechselwirkungen mit dem Atomkern und den umgebenden, gebundenen Elektronen, wobei eine direkte Energieübertragung stattfindet und daraus resultierend die Austrittsenergie geringer als die Eintrittsenergie ist. Die übertragene Stoßenergie führt zur Ionisierung der inneren Schale, das herausgeschlagene Elektron (secondary electron, SE) kann die Probe verlassen. Auf der Detektion dieser Sekundärelektronen basiert ebenfalls die Rasterelektronenmikroskopie. Die innere Schale wird mit einem Elektron höherer Energie aus einer weiter außen liegenden Schale aufgefüllt. Die Energiedifferenz wird durch Emission von Röntgenstrahlung oder Augerelektronen ausgeglichen. Die Energie der abgestrahlten Röntgenstrahlung ist für jedes chemische Element charakteristisch, weshalb die Messung eines Spektrums energiedispersiver Röntgenstrahlung (energy dispersive X-ray, EDX) Informationen über die chemische Zusammensetzung der Probe liefert.

Für die Bilderzeugung werden verschiedene Detektoren eingesetzt, welche die unterschiedlichen Signaltypen erfassen (Abbildung 3.12). Für SEM-Aufnahmen werden Sekundärelektronen beispielsweise mit einem inlens-Detektor oder einem SE2-Detektor gemessen, für die Abbildung von Rückstreuelektronen stehen wiederum eigene Detektoren zur Verfügung. ${ }^{86}$ Der inlens-Detektor erlaubt die Wiedergabe von Informationen bezüglich Morphologie, Oberflächentopografie sowie elektronischer Unterschiede mit hoher lateraler Auflösung. Bei herkömmlichen SE-Detektoren stehen dahingegen die topografischen Informationen im Vordergrund. 

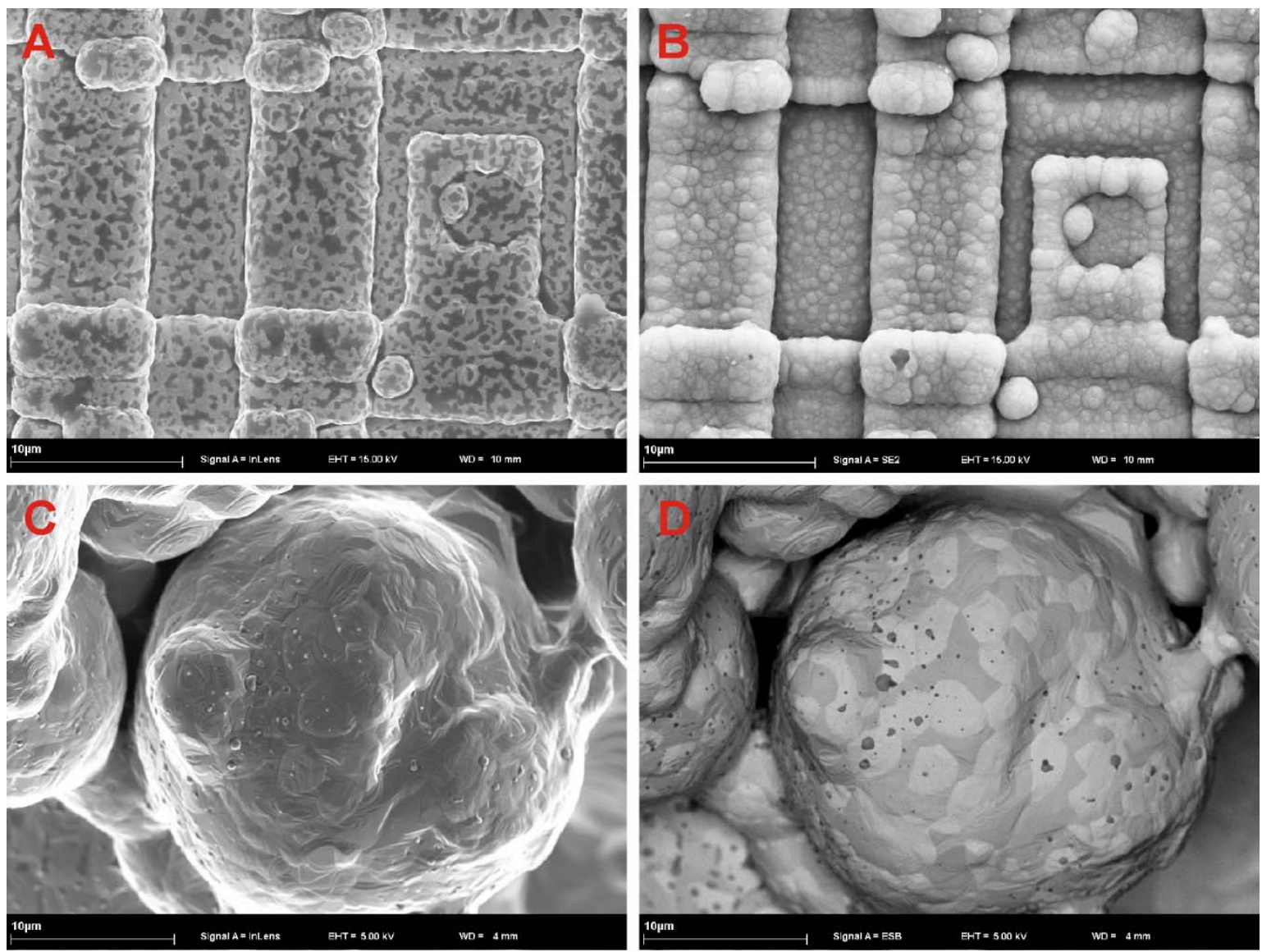

Abbildung 3.12 Vergleich von A inlens-Detektor mit B SE2-Detektor: In A ist die Oberflächenstruktur mit geringem Topografiekontrast erkennbar, während in B die topografischen Informationen dominieren. Vergleich von C SE- mit D BSE-Detektor: Aus C sind gute Oberflächeninformationen erhältlich, in D dahingegen ist deutlich der Materialkontrast sichtbar. ${ }^{86}$

Bei der Transmissionselektronenmikroskopie wird zwischen Hell- und Dunkelfeldmikroskopie unterschieden (bright field, BF beziehungsweise dark field, DF; Abbildung $3.13^{84}$ ), wobei der Unterschied darin besteht, dass in der Hellfeldmikroskopie nur der direct beam

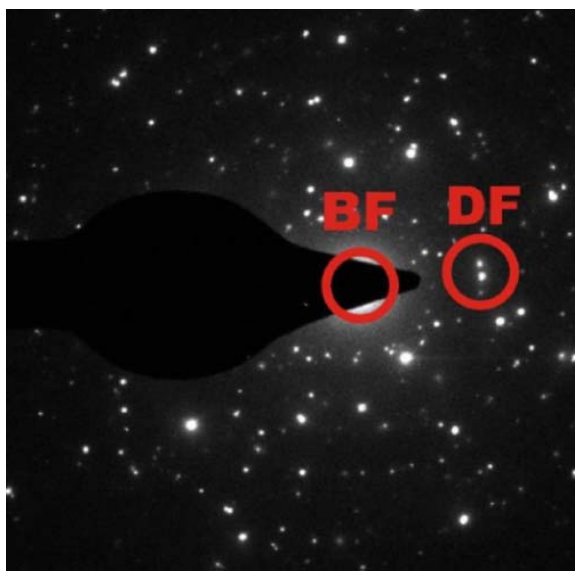

Abbildung 3.13 Elektronenbeugungsmuster: Die Apertur wird entweder um den direct beam (bright field-Bild, BF) oder im Bereich gebeugter Strahlen (dark field-Bild, DF) lokalisiert. gemessen wird. Hierbei erscheinen Bereiche großer Dicke und Materialdichte dunkel, es wird ein Massendickenkontrast erzeugt. Für die Erzeugung von Dunkelfeldabbildungen wird der direct beam ausgeblendet, wodurch ausschließlich gebeugte Strahlen auf den Detektor treffen. Zudem besteht die Möglichkeit, mit dem Elektronenstrahl die Probe abzurastern, diese Methode wird STEM (scanning transmission electron microscopy) genannt. Sie erlaubt die direkte Kombination mit Analysetechniken wie EDX, wodurch Elementkarten ermittelt werden können. 


\section{Durchführung der SEM-Messungen}

Die Silicapräzipitate, die bei der Reaktion von Kieselsäure mit Polyaminen oder Aminolipidvesikeln entstanden, wurden, wie in Abschnitt 3.2.4 erläutert, behandelt. Nach mehrmaligem Waschen wurden jeweils $3 \mu \mathrm{L}$ des Pellets auf einen zuvor polierten Aluminium-SEM-Probenteller aufgetropft. Die Präzipitate, deren Bildung mittels Lichtstreuung verfolgt wurde (Abbildung 5.5), wurden nicht zentrifugiert und gewaschen, stattdessen wurden direkt aus der Messzelle $3 \mu \mathrm{L}$ der Suspension entnommen und auf dem SEM-Träger platziert. Die Proben wurden mindestens einen Tag getrocknet, bevor sie vermessen wurden.

Für die Abbildung der planaren Oberflächen (Kapitel 6.1.2) wurden die Siliciumwafer mit leitenden Graphit-Polycarbonat-Tabs auf die Probenteller aufgeklebt. Die Probenoberfläche wurde mit dem Aluminiumträger mit Hilfe von Silberleitlack kontaktiert.

Die SEM-Messungen wurden an einem Zeiss Leo Supra 55VP-SEM mit einer Hochspannung von $5,00 \mathrm{kV}$ durchgeführt. Zumeist wurde ein inlens-Detektor verwendet, teilweise auch ein Mischsignal vom inlens- und SE2-Detektor erzeugt.

\section{Auswertung der SEM-Aufnahmen}

Den Abbildungen der Silicastrukturen, die bei der Reaktion mit Polyaminen gewonnen wurden, wurde mittels der Software AxioVision (Zeiss) manuell eine Skalierung zugewiesen. Hierzu wurde der mit jedem Bild aufgenommene Maßstabsbalken herangezogen. Danach konnten die Durchmesser der Partikel mit einem Messwerkzeug ermittelt und anschließend ausgegeben werden. Die statistischen Histogrammanalysen der Durchmesser wurden mit der Software OriginPro durchgeführt. Zunächst musste die ideale Binbreite für jede Verteilung ermittelt werden. Dazu wurde die Regel nach Freedman und Diaconis angewendet ${ }^{87}$ :

$$
h=2 \frac{Q_{0,75^{-}} Q_{0,25}}{n^{1 / 3}}
$$

Hierbei ist $h$ die Binbreite und $n$ ist die Anzahl an Ereignissen. $Q_{p}$ ist jeweils ein $p$-Quantil, was bedeutet, dass $Q_{0,25}$ der Durchmesser ist, unterhalb dessen die Durchmesser von $25 \%$ der Kugeln einer Verteilung liegen. Dementsprechend besitzen 75 \% der Kugeln einer Verteilung einen Durchmesser der kleiner als $Q_{0,75}$ ist. $Q_{0,25}$ und $Q_{0,75}$ sind (zusammen mit $Q_{0,5}$ ) die in der Statistik am häufigsten verwendeten Quantilen, sie werden auch Quartile (Viertelwerte) genannt. Die Differenz $Q_{0,75}-Q_{0,25}$ ist auch bekannt als Interquartilabstand (IQR); 
innerhalb des IQR liegen $50 \%$ aller Messwerte. Der IQR kann mit OriginPro bestimmt und daraus die Binbreite berechnet werden. Die Häufigkeit der Ereignisse wird mit dieser Binbreite als Schrittgröße gezählt. Die hieraus erhaltenen Daten wurden mittels einer oder mehrerer Normalverteilungen angepasst, woraus Mittelwerte sowie die Halbwertsbreiten der Verteilungen ermittelt wurden. Die beschriebene Methode der Binbreitenbestimmung eignet sich nur für nicht-überlagerte Verteilungen.

Um zu überprüfen, ob sich die Verteilungen der Kugeldurchmesser verschiedener Proben signifikant voneinander unterscheiden, wurde ein nicht-parametrischer (parameterfreier) Test angewendet. Für nicht-parametrische Tests ist es grundsätzlich nicht nötig, dass die Daten normalverteilt sind, was bei parametrischen Tests wie t-Tests (z. B. Student-tVerteilung) wichtig ist. Ein solcher parameterfreier Test ist der Mann-Whitney-U-Test ${ }^{88,89}$ (äquivalent zu Mann-Whitney-Wilcoxon- und Wilcoxon-Rangsummentest), der für die Analyse im Rahmen dieser Arbeit gewählt wurde. Er kann entweder im Internet ${ }^{90}$ oder mit der Software OriginPro (integriert ab Version 8) durchgeführt werden.

Es werden grundsätzlich zwei Stichproben A und B (in diesem Fall jeweils die Werte der Kugeldurchmesser zweier Proben) definiert und eine Hypothese $\mathrm{zu}$ diesen Proben aufgestellt. Diese Hypothese besagt entweder A $<$ B oder A $>$ B beziehungsweise $A=B$ (Nullhypothese). Daraufhin werden die Daten der beiden Proben hinsichtlich dieser Hypothesen geprüft. Im Rahmen dieser Arbeit wurde die Nullhypothese gewählt. Ist die Hypothese gültig, ist also $\mathrm{A}=\mathrm{B}$, sind die beiden Stichproben wahrscheinlich nicht voneinander unterschiedlich, gehören also einer Verteilung an. Ist die Hypothese jedoch nicht erfüllt, bedeutet dies, das sich die Proben signifikant voneinander unterscheiden, dass also Unterschiede bezüglich der Kugeldurchmesser nicht zufällig sind.

Der dabei erhaltene p-Wert $(P)$ gibt die Wahrscheinlichkeit an, mit der auch eine gültige Nullhypothese zufällig den gleichen Versuchsausgang liefern kann. Je kleiner $P$, desto sicherer ist die Nullhypothese nicht erfüllt. Gebräuchliche angewendete Grenzen sind $P<0,05$ (signifikant) oder $P<0,01$ (hoch signifikant). Werden $\mathrm{p}$-Werte kleiner als diese errechnet, wird die Nullhypothese als nicht erfüllt angesehen.

\section{Durchführung der TEM-, STEM- und EDX-Messungen}

Nach dem Waschen der Polyamin-Silicaproben wurden $3 \mu \mathrm{L}$ mit einer Pipette auf $10 \mathrm{~nm}$ TEM-grids (feinmaschiges Kupfernetz, beschichtet mit einem $10 \mathrm{~nm}$ dicken amorphen Kohlenstofffilm) aufgebracht. Alle Messungen wurden von M. Seibt (IV. Physikalisches 
Institut, Universität Göttingen) an einem Philips CM200-FEG-UT bei einer Spannung von $200 \mathrm{kV}$ durchgeführt.

Die EDX-Elementbilder wurden mit der Software ImageJ dahin gehend überarbeitet, dass sie je nach Element spezifisch eingefärbt und anschließend überlagerte Abbildungen erzeugt wurden. Die Bestimmung der Schalendicken wurde mit der Software AxioVision (Zeiss) durchgeführt. Hierbei wurde jedem Bild anhand des Maßstabsbalkens manuell eine Skalierung zugewiesen, um die tatsächlichen Größen der Objekte korrekt ermitteln zu können.

\subsubsection{Dynamische Lichtstreuung}

Die Dynamische Lichtstreuung (DLS) dient der Ermittlung der Größe, genauer des hydrodynamischen Radius von Partikeln in Suspensionen. Trifft elektromagnetische Strahlung auf Partikel, die kleiner sind als die Lichtwellenlänge, kommt es zur Streuung. In einem optisch ideal homogenen Medium (bei ortsunabhängiger Dielektrizitätszahl $\varepsilon$ ) würde die Streustrahlung am nächsten Streuzentrum durch destruktive Interferenz ausgelöscht werden, eine Ausbreitung der Lichtwelle fände ausschließlich in Vorwärtsrichtung statt. In realen Medien liegen jedoch lokale Inhomogenitäten des Brechungsindexes vor, weshalb Teile der Streustrahlung aus dem Medium austreten. ${ }^{91}$ Praktisch tritt also dann Lichtstreuung auf, wenn ein Brechungsindexunterschied von disperser Phase (Partikel, hier Vesikel oder Mizelle) und Dispergiermedium (Flüssigkeit, hier Puffer) vorliegt. Bei komplexen Flüssigkeiten wie verdünnten, binären Gemischen kommt ein weiterer Streumechanismus hinzu. Aufgrund der Brown'schen Molekularbewegung diffundiert die gelöste Substanz im Umgebungsmedium. Aus den Konzentrationsschwankungen resultieren Schwankungen des Brechungsindexes. Das Spektrum des gestreuten Lichts spaltet sich symmetrisch um $\omega_{0}$ in drei Linien auf, einer lorentzförmigen RayleighLinie um die Kreisfrequenz $\omega_{0}$ sowie zwei Seitenlinien (Brillouin-Komponenten) um $\left(\omega_{0}-\Delta \omega\right)$ beziehungsweise $\left(\omega_{0}+\Delta \omega\right)$ (Abbildung 3.14 A). Die Halbwertsbreite $\Gamma$ der Lorentzkurve steht in Zusammenhang mit dem Diffusionskoeffizienten $D$ :

$$
\Gamma=q^{2} \cdot D
$$


Hierbei ist $q$ der Betrag des Streuvektors:

$$
q=\frac{4 \pi n}{\lambda} \cdot \sin \left(\frac{\theta}{2}\right)
$$

$\theta$ ist der Winkel, bei dem das gestreute Licht detektiert wird, $\lambda$ die Wellenlänge des Lichts, $T$ die Temperatur und $n$ der Brechungsindex des Dispergiermediums. Nach der StokesEinstein-Beziehung ${ }^{92}$ ist der Diffusionskoeffizient $D$ mit dem hydrodynamischen Radius $r_{\mathrm{H}}$ und der Lösungsmittelviskosität $\eta$ nach Gl. 3-4 verknüpft:

$$
D=\frac{k_{B} \cdot T}{6 \pi \cdot \eta \cdot r_{\mathrm{H}}}
$$

$k_{B}$ steht für die Boltzmann-Konstante. Je größer ein Teilchen ist, desto kleiner ist sein Diffusionskoeffizient und desto kleiner ist laut Gl. 3-2 die Halbwertsbreite $\Gamma$ (Abbildung $3.14 \mathrm{~B})$.
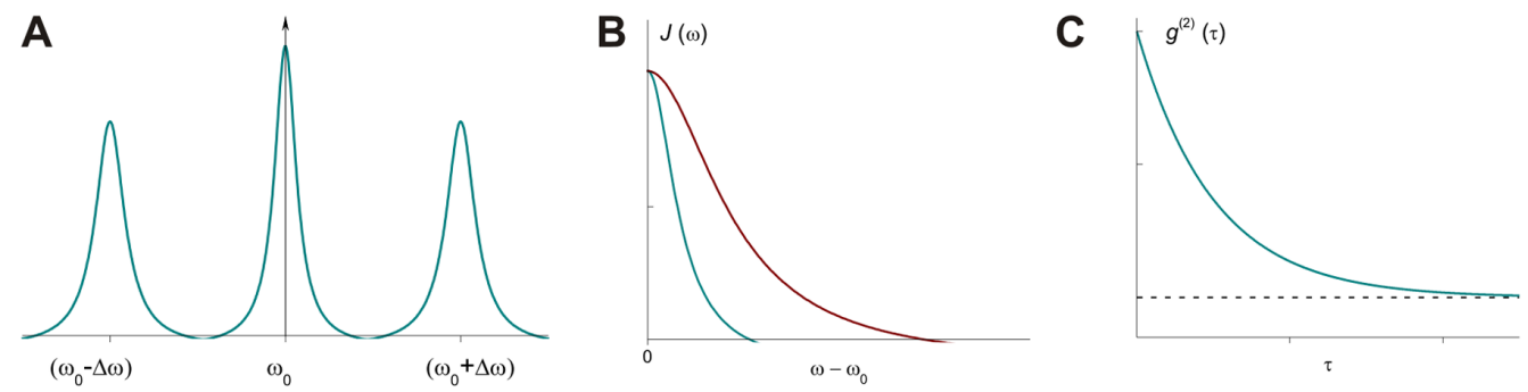

Abbildung 3.14 A Streuspektrum der dynamischen Lichtstreuung mit der lorentzförmigen Rayleigh-Linie und den Brillouin-Komponenten. B Vergleich der optischen Spektren $J(\omega)$ in Anwesenheit von - langsamen großen und - schnellen kleinen Partikeln C Korrelationsfunktion $g^{(2)}(\tau)$ der Intensität.

Prinzipiell kann ein optisches Spektrum mit einem Interferometer gemessen werden, jedoch sind die Frequenzverschiebungen im Vergleich zur Wellenlänge des eingestrahlten Lasers sehr klein, was eine praktische Umsetzung erschwert. Man bedient sich deshalb der Fourier-transformierten Form des Frequenzspektrums, der sogenannten Autokorrelationsfunktion $g^{(1)}(\tau)$. Den Zusammenhang zwischen optischem Spektrum $J(q, \omega)$ und der Autokorrelationsfunktion 1. Ordnung $g^{(1)}(\tau)$ gibt das Wiener-Khintchine-Theorem ${ }^{93,94}$ wider:

$$
J(q, \omega)=\int_{0}^{\infty} g^{(1)}(\tau) \cdot \mathrm{e}^{-i \omega t} \mathrm{~d} \tau
$$

( $\tau$ : Abklingzeit, $t$ : Zeit). Danach ist die lorentzförmige Rayleigh-Linie äquivalent mit dem exponentiellen Abfall der Autokorrelationsfunktion in einem Zeitbereich mit charakteristischer Abklingzeit $\tau$. 
Nach dem Wiener-Khintchine-Theorem (Gl. 3-5) gilt:

$$
\tau=\frac{1}{\Gamma}
$$

In der Praxis wird nicht die Autokorrelationsfunktion $g^{(1)}(\tau)$ (mit der Energie $E$ des elektrischen Feldes), sondern die zeitliche Schwankung der Streuintensität gemessen, aus der sich die Intensitätsautokorrelationfunktion 2. Ordnung $g^{(2)}(\tau)$ (Abbildung 3.14 C)

$$
g^{(2)}(\tau)=\frac{\langle I(t) \cdot I(t+\tau)\rangle}{\left\langle|I|^{2}\right\rangle}
$$

mit der Intensität $I$ ergibt. Die Funktion $g^{(1)}(\tau)$ kann aus $g^{(2)}(\tau)$ berechnet werden. Aus der Messung der Abklingkonstanten $\tau$ der Autokorrelationsfunktion kann somit die Halbwertsbreite des Spektrums und über Gl. 3-2 der Diffusionskoeffizient bestimmt werden. Hieraus wiederum ergibt sich die Möglichkeit den hydrodynamischen Radius $r_{\mathrm{H}} \mathrm{zu}$ ermitteln (Gl. 3-4). Es ergibt sich im Ganzen folgender Zusammenhang zwischen hydrodynamischem Radius und Abklingzeit:

$$
r_{\mathrm{H}}=\frac{k_{B} \cdot T \cdot \tau}{6 \pi \cdot \eta} \cdot\left(\frac{4 \pi n}{\lambda}\right)^{2} \cdot \sin ^{2}\left(\frac{\theta}{2}\right)
$$

Im Rahmen der vorliegenden Arbeit wurden Lipidsuspensionen der Aminolipide vermessen, um zu untersuchen, ob Lipidaggregate vorliegen und gegebenenfalls ihre Größen zu bestimmen und als Schlussfolgerung die Aggregatform (Vesikel oder Mizelle) zu ermitteln (Abschnitt 5.2). Desweiteren wurden Silicapräzipitationsreaktionen an diesen Vesikeln zeitaufgelöst beobachtet (Abschnitt 5.3).

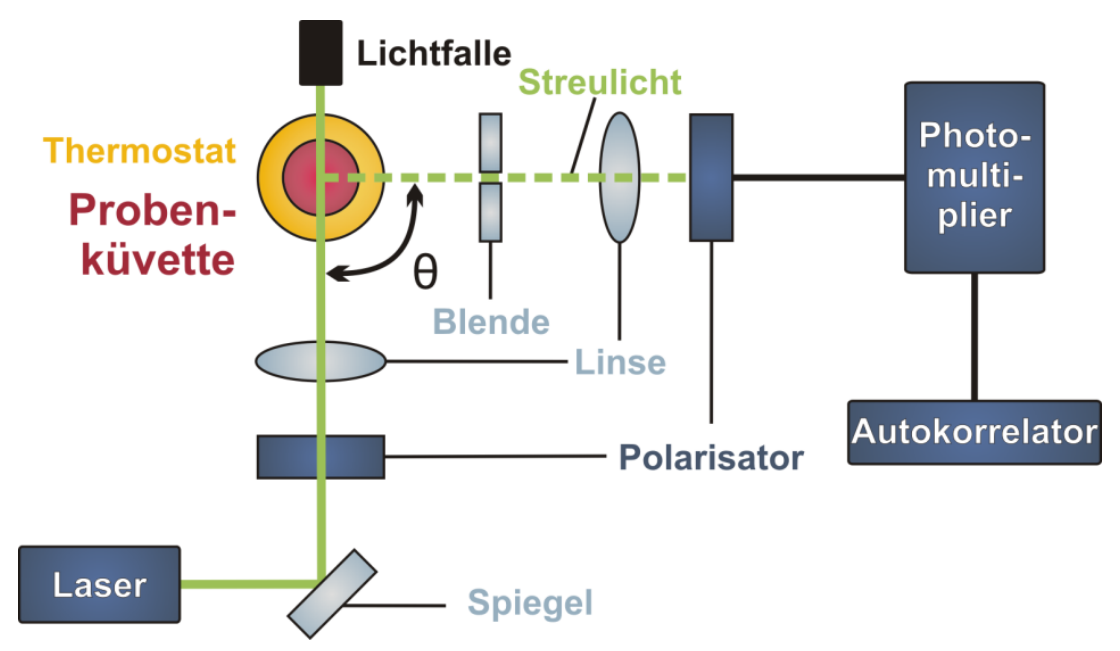

Abbildung 3.15 Schematischer Aufbau der Lichtstreuanlage. 
Es wurde ein Messaufbau (Abbildung 3.15) eingesetzt, bei dem das Streulicht in einem Winkel von $\theta=90^{\circ} \mathrm{C}$ zur Richtung des Laserlichtes (Wellenlänge: $\lambda=532,1 \mathrm{~nm}$ ) gemessen wurde. Bei den Messungen wurde die Temperatur der Probe konstant bei $T=25^{\circ} \mathrm{C}$ gehalten. Für den Brechungsindex des Puffers wurde $n=1,33$ und für die Viskosität $\eta=8,945 \cdot 10^{-4} \mathrm{~kg} \mathrm{~m}^{-1} \mathrm{~s}^{-1}$ eingesetzt.

\subsubsection{Filmwaage}

Mittels der auf Langmuir ${ }^{95}$ und Pockels ${ }^{96}$ zurückgehenden Filmwaagetechnik können die Eigenschaften von Monoschichten amphiphiler Moleküle an einer Grenzfläche (beispielsweise Wasser/Luft) untersucht werden. Messgröße ist hierbei der Oberflächendruck $\pi$, der der Differenz zwischen der Oberflächenspannung des reinen Wassers ohne Film $\left(\gamma_{W}\right)$ und der filmbedeckten Wasseroberfläche $\left(\gamma_{\mathrm{F}}\right)$ entspricht:

$$
\pi=\gamma_{\mathrm{W}}-\gamma_{\mathrm{F}}
$$

Der Oberflächendruck wird mit der Vertikalplattenmethode nach Wilhelmy ermittelt. Messgröße ist die Kraft $F$, die auf das Plättchen wirkt und die proportional zum Oberflächendruck $\pi$ ist. Während der Messung wird bei konstanter Temperatur mittels beweglicher Barrieren die Fläche verändert (Abbildung 3.16), die der Monoschicht und damit den einzelnen Molekülen zur Verfügung steht, wodurch deren Kompressionseigenschaften untersucht werden können. Ergebnis sind charakteristische Schub-FlächenDiagramme ( $\pi / A$-Isothermen). Diese können je nach Kettenlänge und Kopfgruppe der Substanz, pH-Wert und Ionenstärke der Subphase sowie durch Variation der Temperatur sehr unterschiedlich verlaufen und es können verschiedene Phasen und Phasenübergänge auftreten.

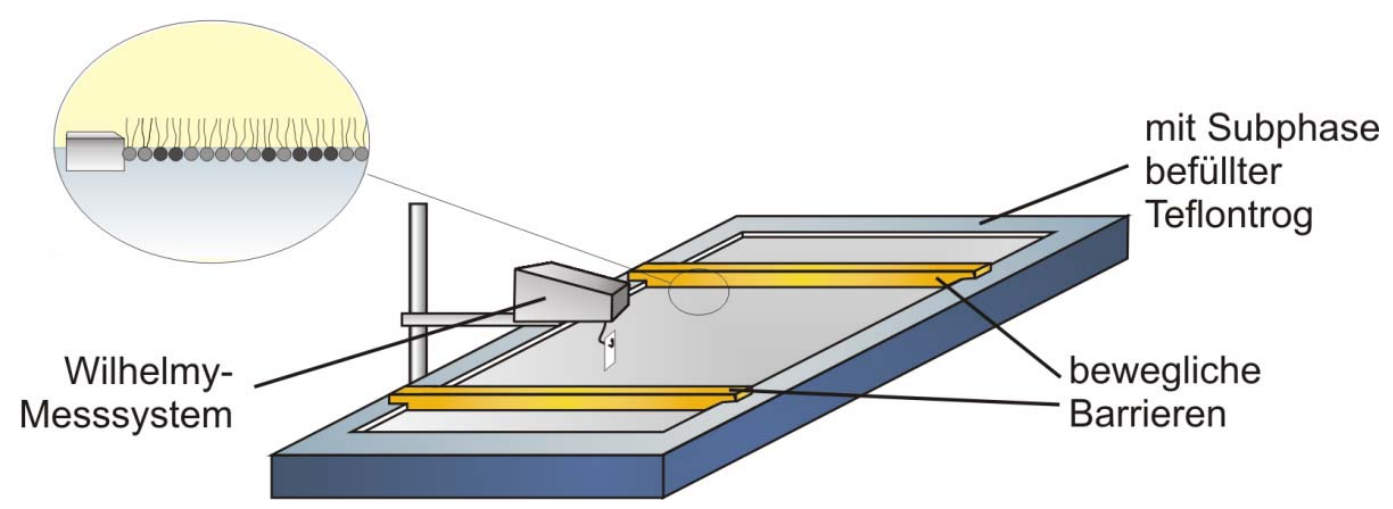

Abbildung 3.16 Schematischer Aufbau einer Filmwaage mit Wilhelmymesssystem. 
In Abbildung 3.17 ist eine idealisierte Isotherme dargestellt, in welcher typische Phasen von Monoschichten erkennbar sind. Steht den Molekülen eine große Fläche zur Verfügung, befindet sich der Film in der gasanalogen Phase (g, gaseous). Die Moleküle haben großen Abstand zueinander und die mittlere Wechselwirkungsenergie ist gering. Bei Verkleinerung der Fläche pro Molekül wird zunächst ein Koexistenzbereich von $g$ - und flüssig-expandierter Phase (liquid-expanded, $l_{e}$ ) erreicht, bevor die Monoschicht in die $l_{e^{-}}$ Phase übergeht. Die hydrophoben Ketten richten sich auf, sie sind noch frei beweglich, jedoch stehen die Moleküle nun in Wechselwirkung miteinander und der Oberflächendruck erhöht sich aufgrund von Abstoßungskräften. Bei weiterer Kompression richtet sich dann ein größerer Teil der Ketten auf und es kommt zu einem weiteren Phasenübergang.

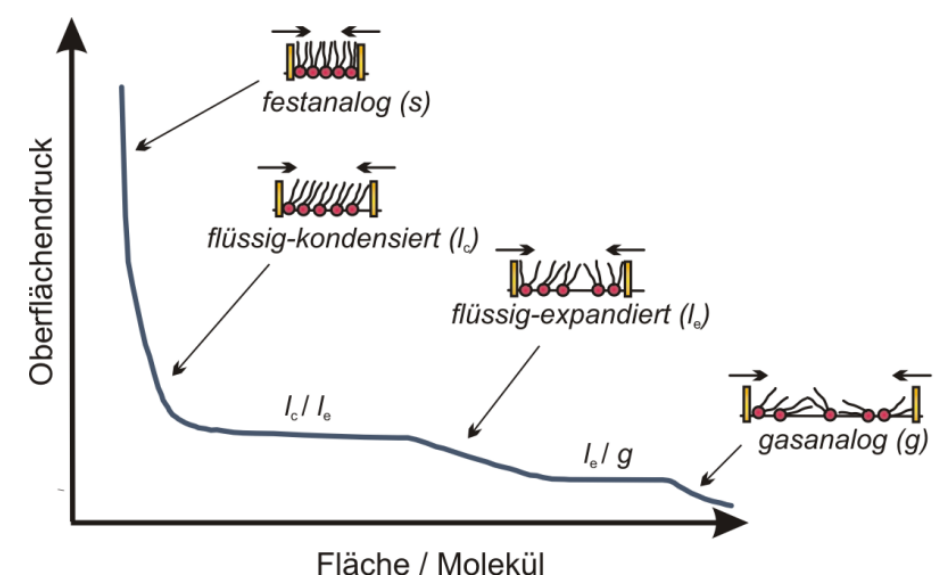

Abbildung 3.17 Idealisiertes Schub-Flächendiagramm einer Lipidmonoschicht an der Wasser/LuftGrenzfläche.

Das Plateau in der Isotherme kennzeichnet einen Koexistenzbereich von $l_{e^{-}}$und flüssigkondensierter Phase (liquid-condensed, $l_{c}$ ). Die Monoschicht besitzt in diesem Bereich große Kompressibilität, der Oberflächendruck steigt nur leicht an, bis der Übergang zur $l_{c}$-Phase erreicht ist. In diesem Bereich sind die Moleküle starr angeordnet und die Alkylketten sind parallel zueinander aufgerichtet. Bei weiterer Verkleinerung der Fläche erfolgt der Übergang in den festanalogen Zustand (solid, s), in welchem die Packungsdichte maximal ist und die Kopfgruppen weitestgehend dehydratisiert sind. Wird die Kompressibilitätsgrenze des Films überschritten, kollabiert dieser. Moleküle tauchen dabei in dreidimensionalen Aggregaten in die Subphase ab, was zu irreversiblem Materialverlust führt.

Die Filmwaage wurde in dieser Arbeit zur Aufnahme von Kompressionsisothermen eingesetzt, aber auch für fluoreszenzmikroskopische Messungen sowie zur Präparation von Langmuir-Blodgett-Filmen (Abschnitt 3.2.3) verwendet. Zur Erzeugung von Mono- 
schichten wurde zunächst der Teflontrog mit Reinstwasser gefüllt und konstant temperiert. Die zu vermessenden Substanzen wurden in Lösungsmittel gelöst, gegebenenfalls wurden Substanzmischungen aus diesen Stammlösungen hergestellt. Nach Auftragen der Lösungen auf die Subphase mittels einer Mikroliterspritze wurde dreißig Minuten gewartet, in welcher Zeit das Lösungsmittel verdunstet und sich das System äquilibriert. Anschließend wurde der Film durch Vorschub der Teflonbarrieren komprimiert und zugleich der Oberflächendruck $\pi$ gemessen.

\subsubsection{Fluoreszenzmikroskopie}

Atome oder Moleküle, die sich in einem elektronisch angeregten Zustand befinden, können beim Übergang in niedrigere Energiezustände spontan Strahlung emittieren. Im Fall von Fluoreszenz erfolgen Übergänge zwischen Zuständen mit gleichen Spins. Sie erfolgt bei einer kleineren Frequenz als die Absorption, da die Emission erst stattfindet, nachdem das Molekül einen Teil der Anregungsenergie an die Umgebung abgegeben hat. Aus diesem Grund ist das Emissionsspektrum im Vergleich zum Absorptionsspektrum zu größeren Wellenlängen hin verschoben (Stokes-Verschiebung).

In Fluoreszenzmikroskopen wird dieses Phänomen genutzt. Im Rahmen dieser Arbeit wurde ein Epifluoreszenzmikroskop verwendet, dessen Aufbau in Abbildung 3.18 schematisch dargestellt ist.

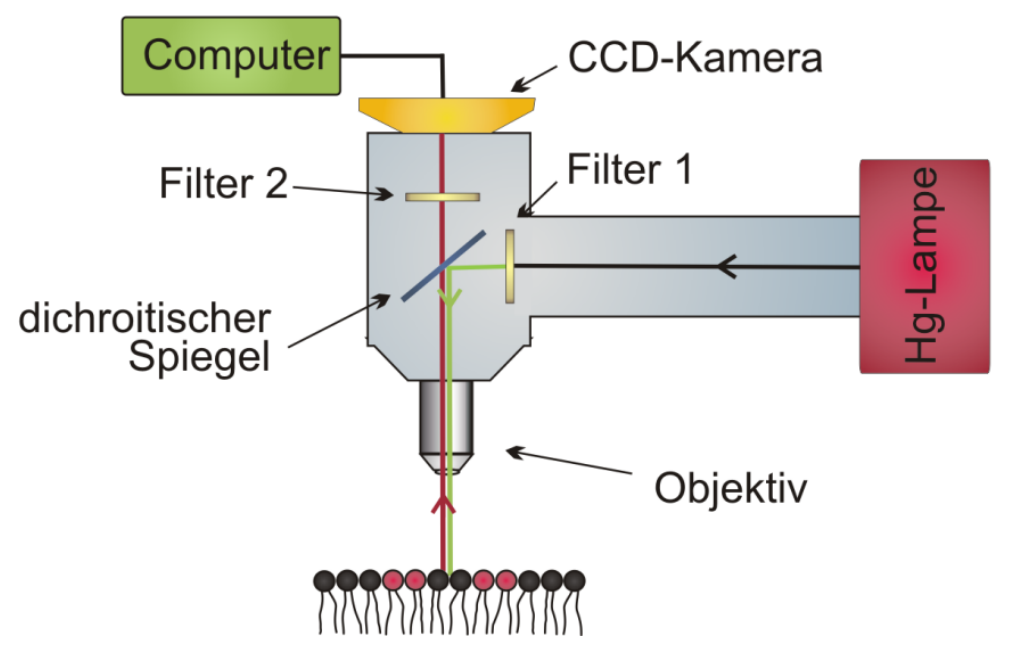

Abbildung 3.18 Schematischer Aufbau eines Epifluoreszenzmikroskops. 
Als Lichtquelle dient eine Quecksilberdampflampe $(\lambda=270$ - $600 \mathrm{~nm})$. Ein Bandpassfilter (Filter 1) ist für Licht mit den zur Anregung nötigen Wellenlängen durchlässig. Der dichroitische Spiegel lenkt das Licht in Richtung des Objektivs und damit zur Probe. Von der Probe emittierte Strahlung kann den dichroitischen Spiegel in Folge ohne Ablenkung passieren. Bevor das Licht auf eine CCD-Kamera trifft, welche mit einem Computer verbunden ist, passiert es einen zweiten Bandpassfilter (Filter 2).

In dieser Arbeit wurden zwei verschiedene Filtersätze verwendet. Filtersatz 44 (Zeiss, Göttingen) beinhaltet einen Bandpassfilter für die Anregung im Bereich von (455 - 495) nm, emittierte Strahlung wird nur im Bereich von (505 - 555) nm durchgelassen. Filtersatz 45 (Zeiss, Göttingen) filtert das Anregungslicht im Bereich von (540 - 580) nm, die emittierte Strahlung im Bereich von (592,5 - 667,5) nm.

Es wurden sowohl auf Substraten immobilisierte Proben untersucht als auch Lipidmonoschichten an der Wasser/Luft-Grenzfläche. Hierzu wurde eine Filmwaage unter dem Mikroskop platziert. Weiterhin ist zu beachten, dass die Aufnahmen in Abbildungen 6.20 und 6.21 mit einem inversen Epifluoreszenzmikroskop erstellt wurden, welches in einem Aufbau mit einem Rasterkraftmikroskop kombiniert war.

\subsubsection{Rasterkraftmikroskopie}

Die Auflösung ist bei herkömmlichen Mikroskopen beschränkt, da das Auflösungsvermögen durch die Wellenlänge des sichtbaren Lichts limitiert ist. Einen großen Fortschritt brachte die Entwicklung des Rastertunnelmikroskops (scanning tunneling microscope, STM), das atomare Auflösung erlaubt, jedoch auf leitende Oberflächen beschränkt ist. Wie das STM gehört auch das Rasterkraftmikroskop (atomic force microscope, AFM) zu den Rastersondenmikroskopen. Mit dieser Technik können auch nicht leitende Oberflächen mit hoher lateraler und vertikaler Auflösung $(\sim 0,1 \mathrm{~nm})$ abgebildet werden. Da die Messungen sowohl an Luft als auch in wässrigen Medien durchgeführt werden können, ist die Untersuchung der Oberflächenstrukturen biologischer Systeme ermöglicht. Neben Informationen über die Topografie einer Probe können auch solche über Materialeigenschaften gewonnen werden.

\section{Funktionsweise}

Anhand Abbildung 3.19 wird das Funktionsprinzip eines Rasterkraftmikroskops erklärt. Das Messprinzip beruht auf einem mechanischen Abtastvorgang der Probenoberfläche mit 
einer sehr feinen Messspitze, die sich an einem Federbalken (cantilever) befindet. Auf diesen wirken beim Annähern an die Oberfläche auf wenige Ångström durch Wechselwirkungen mit der Probe attraktive oder repulsive Kräfte, die vom Abstand und vom Material abhängen. Während des Abrasterns der Probe in x- und y-Richtung wirken auf den cantilever mikroskopische Kräfte, die zu seiner Verbiegung führen, was eine veränderte Auslenkung des Laserstrahls bewirkt. Diese Veränderung wird über eine Vierquadrantendiode detektiert und als Signal an den Computer weitergegeben. Eine Steuerung über den Controller erlaubt die Neuausrichtung des Piezoelements in z-Richtung, so dass der Istwert einer vom Messmodus abhängigen Regelgröße wieder dem Sollwert entspricht.

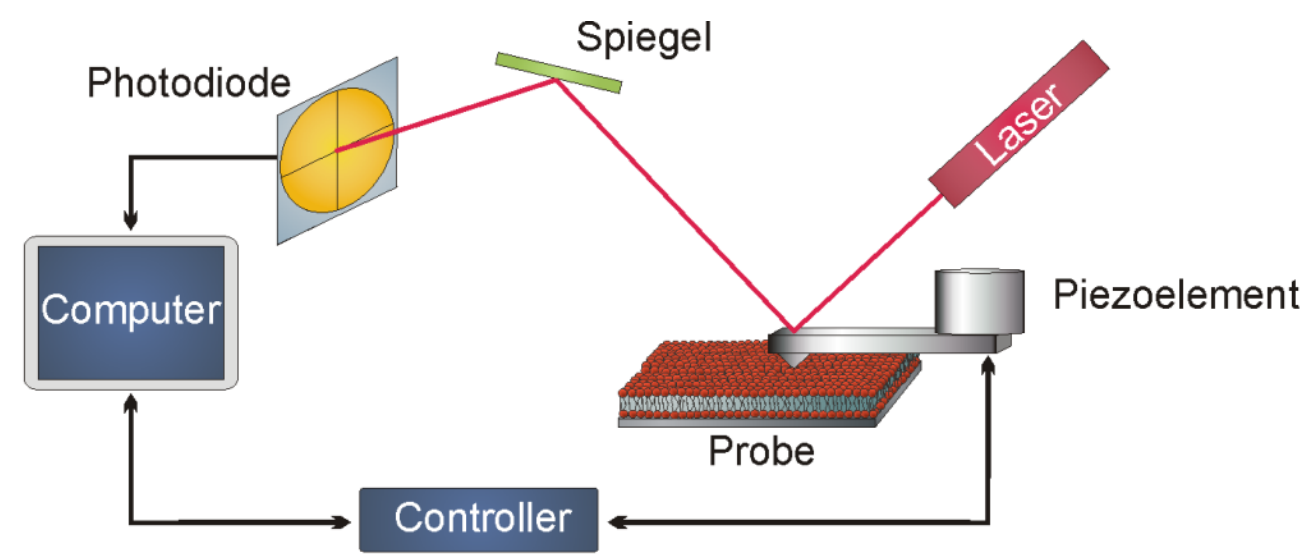

Abbildung 3.19 Schematische Darstellung des Aufbaus eines Rasterkraftmikroskops.

\section{Messmodi}

Je nachdem, welche Regelgröße während einer Messung im System konstant gehalten wird, werden verschiedene Betriebsmodi unterschieden. $\mathrm{Zu}$ den statischen Messmodi wird der contact mode gezählt, bei dem der cantilever in ständigem repulsiven Kontakt mit der Probenoberfläche steht. Mögliche Regelgrößen, die konstant gehalten werden, sind hier die z-Position des Piezoelements (constant height mode) oder die Verbiegung des cantilevers (constant force mode). Da beim constant height-Modus im Gegensatz zum constant forceModus teilweise hohe Kräfte wirken, weil keine Anpassung der z-Position des Piezoelements stattfindet, kann es hierbei zu unerwünschten Manipulationen der Oberfläche kommen. Doch auch im constant force-Modus können große, vor allem laterale Kräfte wirken, wodurch Objekte zur Seite geschoben werden können oder die Spitze in die Probe eindringt, was zu einer verminderten Auflösung führt. 
Zur Minimierung der im contact mode entstehenden Kräfte wurde mit dem tapping mode (auch intermittent contact mode genannt) ein dynamischer Modus entwickelt, der vor allem für die Abbildung biologischer Proben oft dem contact mode gegenüber bevorzugt wird. Der cantilever wird hier durch ein zusätzliches Piezoelement zu einer vertikalen sinusförmigen Schwingung nahe seiner Resonanzfrequenz $f_{0}$ angeregt. Die Messspitze berührt dabei die Probenoberfläche periodisch und durchläuft im Verlauf einer Schwingungsperiode sowohl Bereiche, in denen attraktive Kräfte als auch solche in denen repulsive Kräfte wirken. Regelgröße ist in diesem Fall die Amplitude der Schwingung. Erhöhen sich die zwischen Spitze und Probe wirkenden Kräfte, resultiert daraus eine Dämpfung der Schwingung. Über den Regelkreis wird die z-Position des Piezoelements variiert, bis wieder der Sollwert der Amplitude erreicht ist. Die Information über die Topografie der Oberfläche wird dementsprechend aus der z-Position des Piezoelements gewonnen. Zusätzlich zur Höheninformation liefert der intermittent contact-Modus als Sekundärsignal das Phasenbild. Durch Wechselwirkungen zwischen Probe und Spitze kommt es während der Abbildung zu einer Phasenverschiebung gegenüber dem frei schwingenden System. Das Ausmaß der Phasenverschiebung ist von Materialeigenschaften abhängig, was zu Kontrastunterschieden in der resultierenden Abbildung führt, wenn Bereiche mit unterschiedlichen Eigenschaften vorliegen, auch wenn sie sich in ihrer Höhe nicht unterscheiden.

Einen guten Überblick über weitere Messmodi, die bei der Messung wirkende Kräfte sowie mögliche auftretende Schwierigkeiten bei der Abbildung geben mehrere Dissertationen aus der Arbeitsgruppe. ${ }^{97-99}$

\section{Durchführung}

Die AFM-Messungen wurden an NanoWizard I- und NanoWizard II-Geräten der Firma JPK Instruments (Berlin, Deutschland) durchgeführt. Sofern in Flüssigkeit gemessen wurde, handelte es sich um Reinstwasser, da in den verwendeten MES-Puffern die Messspitze schneller verschmutzte. Alle abgebildeten Aufnahmen wurden im tappingModus erstellt. Für die Messungen in Flüsigkeit wurden entweder Silicium-cantilever CSC37 (nominale Federkonstante $C=0,3 \mathrm{~N} / \mathrm{m}$, Resonanzfrequenz $f_{0}=21 \mathrm{kHz}$, Mikromasch, Tallinn, Estland) oder goldbeschichtete Siliciumnitrid-cantilever MSCT $\left(C=0,5 \mathrm{~N} / \mathrm{m}, f_{0}=120 \mathrm{kHz}\right.$, Veeco, Camarillo, USA) benutzt. Die Experimente an Luft wurden mit Silicium-cantilevern Pointprobe $\mathrm{NCH} \quad\left(C=42 \mathrm{~N} / \mathrm{m}, f_{0}=330 \mathrm{kHz}\right.$, Nanosensors, Neuchatel, Schweiz) durchgeführt. 


\subsubsection{Ellipsometrie}

Die Ellipsometrie ist eine optische Methode, die die Charakterisierung dünner Schichten durch Detektion der Änderung des Polarisationszustandes bei der Reflexion von Licht erlaubt. ${ }^{100,101}$ Es können Brechungsindizes $n$ sowie Schichtdicken $d$ ermittelt werden. Die Messung verändert die Probe nicht und kann auch in wässrigen Medien durchgeführt werden, was sie für biophysikalische Anwendungen interessant macht.

\section{Polarisation des Lichts}

Licht kann als eine sich im Raum ausbreitende elektromagnetische Welle mit einem elektrischen Feldvektor $E$ und einem magnetischen Feldvektor $B$ beschrieben werden. Dabei stehen elektrischer und magnetischer Feldvektor orthogonal zueinander. Im Gegensatz zu natürlichem Licht, bei dem die Schwingungsebenen der Feldvektoren statistisch verteilt sind, besitzt polarisiertes Licht Feldvektoren mit definierter Schwingungsebene. Der elektrische Feldvektor $E$ ergibt sich aus der Überlagerung zweier senkrecht zueinander stehenden polarisierten Teilstrahlen. Diese können als elektrisches Feld senkrecht $E_{\mathrm{s}}$ und parallel $E_{\mathrm{p}}$ zur Einfallsebene des Lichts dargestellt werden. Für die Wellengleichungen ergibt sich:

$$
\begin{gathered}
E_{\mathrm{s}}=E_{\mathrm{s}, 0} \sin (\omega t) \\
E_{\mathrm{p}}=E_{\mathrm{p}, 0} \sin (\omega t+\delta)
\end{gathered}
$$

mit $E_{\mathrm{s}, 0}$ : Amplitude von $E_{\mathrm{s}}, E_{\mathrm{p}, 0}$ : Amplitude von $E_{\mathrm{p}}$, $\omega$ : Kreisfrequenz des schwingenden Feldvektors sowie $\delta$ : Phasenverschiebung der Teilwellen $E_{\mathrm{p}} \mathrm{zu} E_{\mathrm{s}}$.

Es werden drei Formen der Polarisation unterschieden:

Lineare Polarisation: Der durch Überlagerung von $E_{\mathrm{s}}$ und $E_{\mathrm{p}}$ gebildete elektrische Feldvektor $E$ schwingt in einer Ebene mit der Phasenbeziehung $\delta=\mathrm{n} \pi(\mathrm{n}=0,1,2, \ldots)$.

Zirkulare Polarisation: Der elektrische Feldvektor $E$ rotiert kreisförmig mit definierter Kreisfrequenz um den Wellenvektor. Die Amplituden der Teilwellen $E_{s}$ und $E_{p}$ sind gleich $\operatorname{gro} ß\left(E_{\mathrm{s}, 0}=E_{\mathrm{p}, 0}\right)$, jedoch um $\delta=\mathrm{n} \pi / 2(\mathrm{n}=1,3,5, \ldots)$ phasenverschoben.

Elliptische Polarisation: Der elektrische Feldvektor $E$ beschreibt eine elliptische Umlaufbahn um den Wellenvektor. Es wird zwischen zwei Fällen unterschieden: Im ersten Fall sind die Amplituden voneinander verschieden $\left(E_{\mathrm{s}, 0} \neq E_{\mathrm{p}, 0}\right)$ und es besteht eine Phasenverschiebung von $\delta=\mathrm{n} \pi / 2(\mathrm{n}=1,3,4, \ldots)$ vor, im zweiten Fall liegen gleich große Amplituden $\operatorname{vor}\left(E_{\mathrm{s}, 0}=E_{\mathrm{p}, 0}\right)$ und die Phasenbeziehung beträgt $\delta \neq \mathrm{n} \pi / 2$. 


\section{Reflexion und Brechung an Oberflächen}

Trifft ein polarisierter Lichtstrahl an einer Grenzfläche von einem Medium 1 (Brechungsindex $n_{1}{ }^{*}$ ) aus auf ein anderes Medium 2 (Brechungsindex $n_{2}{ }^{*}$ ), so wird er nicht nur reflektiert sondern auch gebrochen (Abbildung 3.20). Sowohl der reflektierte als auch der gebrochene Strahl werden dabei teilpolarisiert. Das Amplitudenverhältnis der reflektierten und gebrochenen Teilwellen für den Übergang 12 ist durch die Fresnel-Reflexionskoeffizienten $r^{\mathrm{p}}$ und $r^{\mathrm{s}}$ gegeben, die lauten:

$$
\begin{gathered}
r_{12}^{\mathrm{s}}=\frac{n_{1}^{*} \cos \phi_{1}-n_{2}^{*} \cos \phi_{2}}{n_{1}^{*} \cos \phi_{1}+n_{2}^{*} \cos \phi_{2}} \\
r_{12}^{\mathrm{p}}=\frac{n_{2}^{*} \cos \phi_{1}-n_{1}^{*} \cos \phi_{2}}{n_{2}^{*} \cos \phi_{1}+n_{1}^{*} \cos \phi_{2}}
\end{gathered}
$$

mit $\phi_{1}$ : Einfallswinkel des Lichts und $\phi_{2}$ : Winkel nach Brechung. Da nur der Einfallswinkel $\phi_{1}$ bekannt ist, muss $\cos \phi_{2}$, falls benötigt, folgendermaßen berechnet werden:

$$
\cos \phi_{2}=\frac{\left(n_{2}^{2}-n_{1}^{2} \sin ^{2} \phi_{1}\right)^{1 / 2}}{n_{2}}
$$

Dies gilt für den häufigen Fall, dass die Medien 1 und 2 transparent und dementsprechend $n_{1}$ und $n_{2}$ nicht komplex sind.

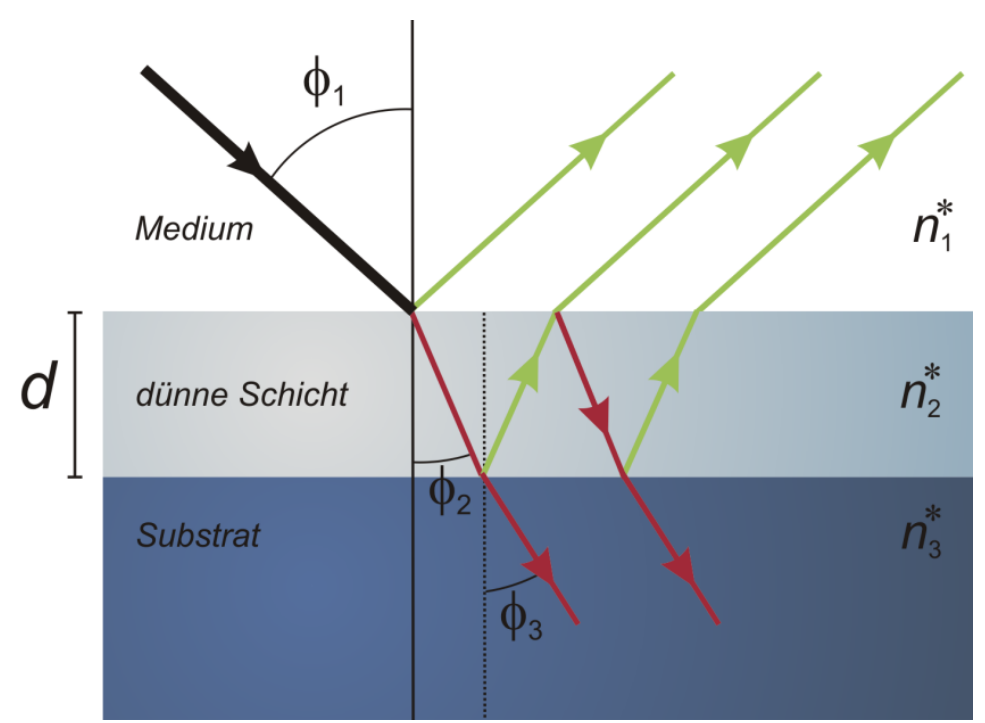

Abbildung 3.20 Reflexion (grüne Pfeile) und Brechung (rote Pfeile) eines Lichtstrahls an zwei Grenzflächen. 
Die Intensität des Lichts ist proportional zum Quadrat der Amplitude. Entsprechend wird die Reflektivität $\mathbb{R}$ (Verhältnis der reflektierten Intensität zu einfallender Intensität) im Fall einer einzelnen Grenzfläche folgendermaßen definiert:

$$
\begin{aligned}
& \mathbb{R}^{\mathrm{s}}=\left|r^{\mathrm{s}}\right|^{2} \\
& \mathbb{R}^{\mathrm{p}}=\left|r^{\mathrm{p}}\right|^{2}
\end{aligned}
$$

Befindet sich eine dünne Schicht $\left(n_{2}{ }^{*}\right)$ auf einem Substrat mit Brechungsindex $n_{3}{ }^{*}$, findet auch an der zweiten Grenzschicht Brechung und Reflexion statt (Abbildung 3.20). Die Fresnel-Koeffizienten $r^{\mathrm{p}}$ und $r^{\mathrm{s}}$ werden analog zu G1. 3-12 beziehungsweise 3-13 für den Übergang 23 definiert. $\cos \phi_{3}$ ergibt sich dann zu:

$$
\cos \phi_{3}=\frac{\left[n_{3}^{* 2}-n_{2}^{2}\left(1-\cos ^{2} \phi_{2}\right)\right]^{1 / 2}}{n_{3}^{*}}
$$

Insgesamt ergeben sich für den Fall einer dünnen Schicht auf einem Substrat folgende Fresnel-Reflexionskoeffizienten:

$$
\begin{aligned}
& R^{\mathrm{s}}=\frac{r_{12}^{\mathrm{s}}+r_{23}^{\mathrm{s}} \mathrm{e}^{-\mathrm{i} 2 \beta}}{1+r_{12}^{\mathrm{s}} r_{23}^{\mathrm{s}} \mathrm{e}^{-\mathrm{i} 2 \beta}} \\
& R^{\mathrm{p}}=\frac{r_{12}^{\mathrm{p}}+r_{23}^{\mathrm{p}} \mathrm{e}^{-\mathrm{i} 2 \beta}}{1+r_{12}^{\mathrm{p}} r_{23}^{\mathrm{p}} \mathrm{e}^{-\mathrm{i} 2 \beta}}
\end{aligned}
$$

Hierbei steht $\beta$ für die optische Weglänge, die in einem Zusammenhang mit der Schichtdicke $d$ (Abbildung 3.20) steht:

$$
\beta=2 \pi\left(\frac{d}{\lambda}\right) n_{2}^{*} \cos \phi_{2}
$$

An mehreren Grenzflächen ist die Reflektivität $R$ definiert als:

$$
\begin{aligned}
& R^{\mathrm{s}}=\left|R^{\mathrm{s}}\right|^{2} \\
& \mathbb{R}^{\mathrm{p}}=\left|R^{\mathrm{p}}\right|^{2}
\end{aligned}
$$




\section{Ellipsometrische Grundgleichung}

Die ellipsometrische Messung beruht auf der Messung der beiden ellipsometrischen Winkel Delta und Psi ( $\Delta$ und $\Psi$ ). $\Delta$ beschreibt dabei die Änderung der Phasendifferenz zwischen $E_{\mathrm{s}}$ und $E_{\mathrm{p}}$ der einfallenden Welle $\left(\delta_{1}\right)$ im Vergleich zur Phasendifferenz zwischen $E_{\mathrm{s}}$ und $E_{\mathrm{p}}$ der reflektierten Welle $\left(\delta_{2}\right)$ (Abbildung 3.21):

$$
\Delta=\delta_{1}-\delta_{2}
$$

Delta kann hierbei Werte von $\left(0\right.$ - 360) ${ }^{\circ}$ annehmen.

Des Weiteren ändert sich bei der Reflexion das Betragsverhältnis der Amplituden der Teilwellen (Abbildung 3.21). Diese Änderung lässt sich durch den Winkel $\Psi$ über das Verhältnis der Reflexionskoeffizienten der parallelen und senkrechten elektrischen Feldvektorkomponenten berechnen:

$$
\tan \Psi=\frac{\left|R^{\mathrm{p}}\right|}{\left|R^{\mathrm{s}}\right|}
$$

mit $R^{\mathrm{p}}$ : Reflexionskoeffizient des parallelen und $R^{\mathrm{s}}$ : Reflexionskoeffizient des senkrechten elektrischen Feldvektors (G1. 3-18 und 3-19). Die Parameter werden über die ellipsometrische Grundgleichung miteinander verknüpft:

$$
\tan \Psi \mathrm{e}^{\mathrm{i} \Delta}=\frac{R^{\mathrm{p}}}{R^{\mathrm{s}}}
$$

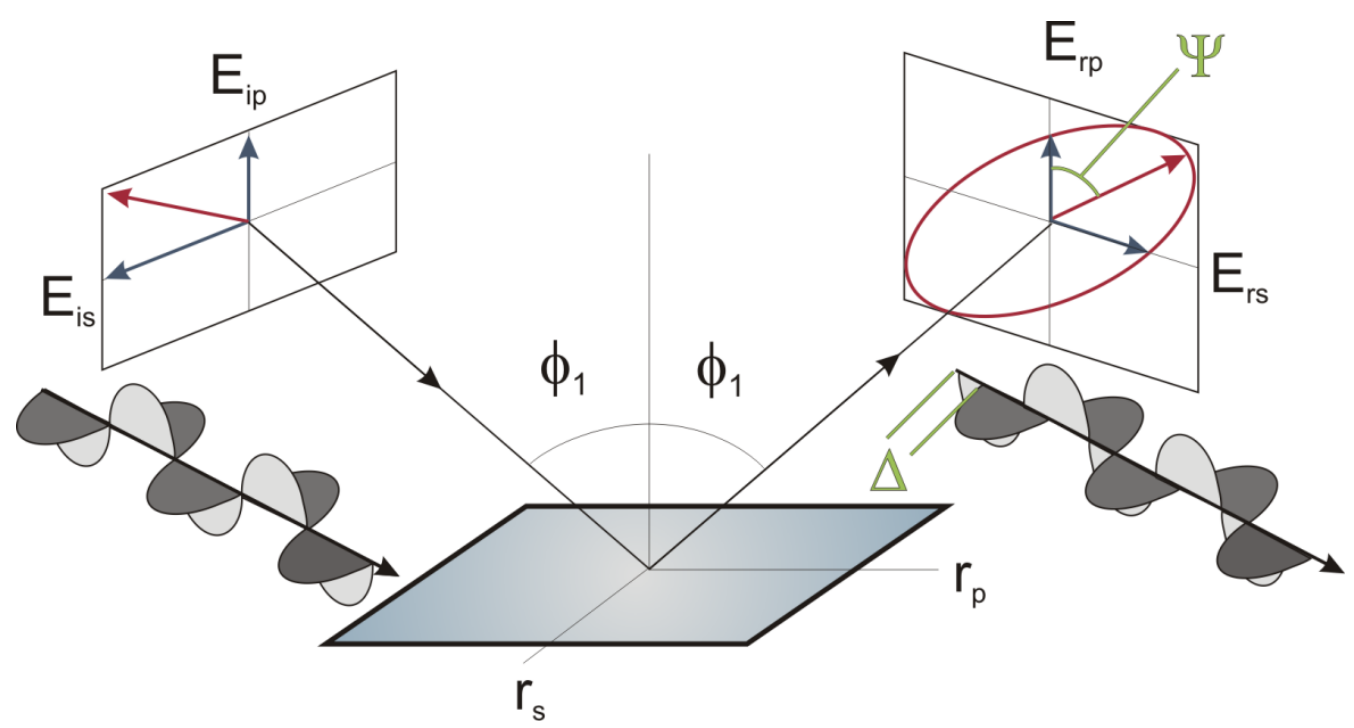

Abbildung 3.21 Schematische Darstellung der Polarisationsänderung der einfallenden elektrischen Feldvektoren $E_{\mathrm{ip}}$ und $E_{\mathrm{is}}$ bei Reflexion. Die ausfallenden elektrischen Feldvektoren $E_{\mathrm{rp}}$ und $E_{\mathrm{rs}}$ weisen gegenüber den einfallenden eine Änderung der Phasendifferenzen $\Delta$ sowie eine Änderung der Amplitude $\Psi$ auf. 


\section{Messaufbau}

In dieser Arbeit wurde ein Präzisionsellipsometer EL X-02C (DRE, Ratzeburg, Deutschland) verwendet, dessen Aufbau in Abbildung 3.22 schematisch dargestellt ist. Als Lichtquelle dient ein Laser einer Wellenlänge von $\lambda=632,8 \mathrm{~nm}$. Das unpolarisierte Licht wird durch Drehung eines Polarisators zunächst linear polarisiert. Mittels eines $\lambda$-Viertelplättchens wird elliptische Polarisation so erzeugt, dass nach der Reflexion (Einfallswinkel $\phi_{1}=70^{\circ}$ ) linear polarisiertes Licht vorliegt. Im Analysator wird das Licht durch Drehung zur Auslöschung gebracht (Nullellipsometer). Durch die jeweilige Stellung des Polarisators beziehungsweise Analysators werden die Winkel $\Delta$ und $\Psi$ ermittelt.

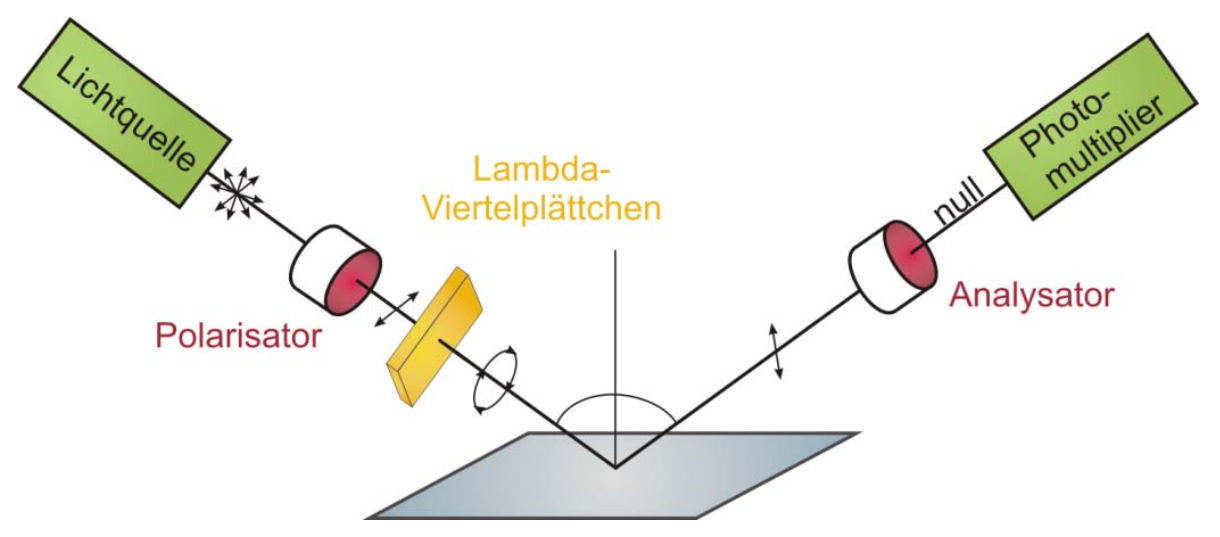

Abbildung 3.22 Schematischer Aufbau eines Nullellipsometers.

Die Messbedingungen finden sich in den entsprechenden Abschnitten zur Präparation (Kapitel 3.2.2 und 3.2.4) und zu den Ergebnissen (Kapitel 6.1.1 und 6.1.2). 


\section{Polyamine}

Die polyamindirigierte Kondensation von Kieselsäure wird durch Phasenseparationsprozesse und Aggregatbildung der Polyamine in wässrigen Lösungen beeinflusst. ${ }^{31}$ Dabei spielen neben der Struktur der Polyamine die Reaktionsbedingungen eine entscheidende Rolle. Die Aggregatbildung wird durch den $\mathrm{pH}-$ Wert, die Konzentration an Polyaminen und die Anwesenheit und Konzentration von multivalenten Anionen bestimmt, und wirkt sich auf anschließende Präzipitationsreaktionen aus. Diese wiederum hängen auch von der Konzentration der Kieselsäure und der Reaktionszeit ab. ${ }^{31,41,65}$ Neuere Untersuchungen mit unverzweigten Polyaminen zeigten, dass sich verschiedene Modifikationen bezüglich Strukturmerkmalen wie Polymerisierungsgrad und damit Kettenlänge, Länge der hydrophoben Alkylgruppen zwischen Aminoeinheiten sowie Amin-Methylierung auf das Präzipitationspotenzial und auf die Morphologie des entstehenden $\mathrm{SiO}_{2}$ auswirken. ${ }^{102-104}$ Dabei wurden bisher nur Polyamine mit bis zu sieben Aminoeinheiten oder große Polymere verwendet. Die long-chain polyamines (LCPA), die in Diatomeen gefunden wurden, weisen jedoch zwischen sieben und 19 Aminoeinheiten pro Molekül auf. ${ }^{3}$ In dieser Arbeit konnten Polyamine mit sechs bis 18 Aminogruppen eingesetzt werden, um einige der zuvor angesprochenen Aspekte systematisch zu untersuchen. Die dabei gewonnenen Erkenntnisse werden im Folgenden näher vorgestellt.

\subsection{Strukturen und Eigenschaften der verwendeten Polyamine}

Insgesamt wurden mit sieben verschiedenen Polyaminen $\mathrm{SiO}_{2}$-Präzipitationen durchgeführt. In Tabelle 4.1 sind verschiedene Informationen $\mathrm{zu}$ den eingesetzten Molekülen zusammengefasst. Neben Gesamtzahl der Aminogruppen pro Molekül $N_{\mathrm{N}}$ und Molarer Masse $M$, ohne Berücksichtigung von Trifluoressigsäure (TFA) als Gegenion zu jeder Aminogruppe, finden sich weiterhin die verwendeten Abkürzungen, die Struktur- sowie die Summenformeln. Die Bezeichnung $\mathrm{C} x \mathrm{~N} y$ gibt jeweils die Anzahl $x$ der $\mathrm{CH}_{2}$-Gruppen zwischen zwei Aminoeinheiten sowie die Gesamtzahl y $\left(=N_{\mathrm{N}}\right)$ der Aminogruppen pro Molekül wieder. Der Zusatz Me weist auf vorliegende $N$-Methylierungen hin.

In der ersten Versuchsreihe wurden die drei Polyamine C3N6, C3N12 und C3N18 (Tabelle 4.1 A-C), die ein identisches $N$-Methylierungsmuster und gleiche Alkylgruppen, nämlich 
jeweils eine Propylgruppe (C3), zwischen den Aminoeinheiten aufweisen, untersucht. Diese Verbindungen unterscheiden sich in der Kettenlänge und damit in der Anzahl an Aminogruppen, von denen Verbindung C3N6 sechs, C3N12 dahingegen zwölf und C3N18 achtzehn besitzt. Davon sind die zwei jeweils enständigen Aminogruppen primär und die restlichen $\left(N_{\mathrm{N}}-2\right)$ sekundär. Die zweite Reihe umfasst drei Polyamine (Tabelle 4.1 D-F), die jeweils zwei primäre und fünf sekundäre Aminogruppen aufweisen, deren Alkylgruppe jedoch variiert. In Verbindung C2N7 liegen jeweils Ethylgruppen vor, während sich in C3N7 Propyl- und in C4N7 Butylgruppen finden. Weiterhin wurde der Einfluss der Methylierung von Aminogruppen mittels Vergleich der Substanzen C3N12Me (Tabelle $4.1 \mathrm{G})$ und C3N12 untersucht. Ihr Aufbau ist identisch, außer dass in C3N12Me alle sekundären Aminoeinheiten methyliert wurden und somit tertiär sind.

Tabelle 4.1 Überblick über die Strukturen der eingesetzten Polyamine.

\begin{tabular}{|c|c|c|c|c|c|}
\hline & Abkürzung & $N_{N}$ & $M I(\mathrm{~g} / \mathrm{mol})$ & Strukturformel & Summenformel \\
\hline A & C3N6 & 6 & 302,5 & & $\mathrm{C}_{15} \mathrm{H}_{38} \mathrm{~N}_{6}$ \\
\hline B & C3N12 & 12 & 645,1 & & $\mathrm{C}_{33} \mathrm{H}_{80} \mathrm{~N}_{12}$ \\
\hline C & C3N18 & 18 & 987,6 & & $\mathrm{C}_{51} \mathrm{H}_{122} \mathrm{~N}_{18}$ \\
\hline D & $\mathrm{C} 2 \mathrm{~N} 7$ & 7 & 275,4 & & $\mathrm{C}_{12} \mathrm{H}_{33} \mathrm{~N}_{7}$ \\
\hline $\mathbf{E}$ & C3N7 & 7 & 359,6 & & $\mathrm{C}_{18} \mathrm{H}_{45} \mathrm{~N}_{7}$ \\
\hline $\mathbf{F}$ & C4N7 & 7 & 443,8 & $\mathrm{H}_{2} \mathrm{~N}^{-}$ & $\mathrm{C}_{24} \mathrm{H}_{57} \mathrm{~N}_{7}$ \\
\hline G & C3N12Me & 12 & 785,3 & & $\mathrm{C}_{43} \mathrm{H}_{70} \mathrm{~N}_{12}$ \\
\hline
\end{tabular}

\subsection{Einfluss der Struktur von Polyaminen auf Präzipitationsreaktionen}

Um den Einfluss der Strukturen von Polyaminen auf die Bildung von amorphem Silica zu untersuchen, wurden zunächst quantitative Analysen mittels der $\beta$-Molybdatmethode (siehe Abschnitt 3.3.1) durchgeführt. Zunächst wurden Stammlösungen der Polyamine 
hergestellt, wozu jeweils eine abgewogene Menge mit einem definierten Volumen an Reinstwasser versetzt wurde, wobei alle Flüssigkeiten klar blieben. Aus der Literatur ist bekannt, dass Polyamine in wässrigem Medium nicht monomer vorliegen, sondern dass auf mikroskopischer Ebene eine Phasenseparation stattfindet und die Polyamine in „Mikrotröpfchen“ vorliegen. ${ }^{32,41}$ Da an dieser Stelle dahin gehend keine Untersuchungen durchgeführt wurden und somit keine konkreten Aussagen $\mathrm{zu}$ den vorliegenden Aggregatformen gemacht werden können, wird nachfolgend vereinfacht von „Polyaminlösungen" gesprochen, anstatt von Emulsionen oder Suspensionen.

Aus der jeweiligen Polyaminstammlösung und Puffer wurden die benötigten Konzentrationen eingestellt und im Anschluss wurde bei den gewünschten Bedingungen monomere Kieselsäure hinzugefügt. Nach genau zehn Minuten wurde das gebildete $\mathrm{SiO}_{2}$ durch Zentrifugation sedimentiert. Diese Zeit erwies sich, wie in Kapitel 4.4 näher erläutert, als ausreichend, um aussagekräftige Ergebnisse sowohl zu Mengen von präzipitiertem Silica als auch dessen mikroskopischen Strukturen ermitteln zu können. Nach mehrmaligem Waschen mit Wasser und anschließendem Zentrifugieren, wurde der Niederschlag mit $1 \mathrm{mM}$ Natronlauge zu monomerer Kieselsäure hydrolysiert, die mit Ammoniumheptamolybdat einen gelben Komplex bildet und über photometrische Messungen eine Ermittlung der Si-Konzentration erlaubt. Die ursprünglich präzipitierte Masse an Silicium kann anschließend berechnet werden.

Alle vorgestellten Ergebnisse wurden, falls nicht anders erwähnt, bei pH 6,8 in $30 \mathrm{~mm}$ Phosphatpuffer durchgeführt. Die Polyamine wurden in Puffer gelöst, sodass die Endkonzentrationen jeweils $1 \mathrm{~mm}$ in Bezug auf die N-Atome betrug. Die Konzentration der frisch hydrolysierten Kieselsäure betrug nach der Zugabe $100 \mathrm{mM}$, womit in allen Proben ein konstantes N:Si-Verhältnis von 1:100 vorlag. Es wurde für zehn Minuten inkubiert, bevor die Proben zentrifugiert wurden. Um Einblicke in die Strukturen des gebildeten Silica zu erhalten, wurden elektronenmikroskopische Analysen durchgeführt. Rasterelektronenmikroskopische (SEM) Aufnahmen bilden die Oberfläche der $\mathrm{SiO}_{2}$ Aggregate $a b$ und geben Auskunft über deren Aussehen, Größe und Verteilung. Dahingegen erlauben transmissionselektronenmikroskopische (TEM) Messungen zusätzlich einen Blick in das Innere der gebildeten Partikel. Daran gekoppelte energiedispersive Röntgenspektroskopie (energy dispersive X-ray spectroscopy, EDX) kann die Verteilung von chemischen Elementen und in Verbindung mit den mikroskopischen Daten ein sehr genaues Bild der Präzipitate liefern. Die hierzu benötigten Präparate wurden analog zu denen, die für die quantitativen Analysen hergestellt wurden, 
erhalten, allerdings wurde der Niederschlag nicht mit Natronlauge gelöst. Stattdessen wurden einige Mikroliter der Suspension auf Aluminium-SEM-Probenträger beziehungsweise auf Kohlenstoff-benetzte Kupfer-TEM-grids pipettiert, mindestens einen Tag getrocknet und anschließend untersucht.

\subsubsection{Kettenlänge der Polyamine}

Um zu untersuchen, inwieweit der Polymerisierungsgrad von linearen Polyaminen die Präzipitationen beeinflusst, wurden die Oligopropylamine C3N6, C3N12 und C3N18 verwendet. Wie zuvor erläutert, weisen die Moleküle gleiche Alkylgruppen auf, womit die Hauptunterschiede in der Anzahl der Aminoeinheiten pro Molekül sowie den Längen der Substanzen liegen. Die mittels $\beta$-Molybdatmethode ermittelten Siliciummassen sind in Abbildung 4.1 zusammengefasst. Die mit 9,0 $\mu \mathrm{g}$ geringste Menge an Silica wurde mit C3N6 präzipitiert. Die Massen steigen mit zunehmender Kettenlänge von 14,0 $\mu \mathrm{g}$ für C3N12 bis auf 22,0 $\mu \mathrm{g}$ mit C3N18 an.

\begin{tabular}{cccc}
\hline & $m_{\mathrm{Si}} / \mu \mathrm{g}$ & $\sigma / \mu \mathrm{g}$ & $n$ \\
\hline C3N6 & 9,0 & 2,6 & 32 \\
C3N12 & 14,0 & 1,5 & 20 \\
C3N18 & 22,0 & 1,7 & 32
\end{tabular}

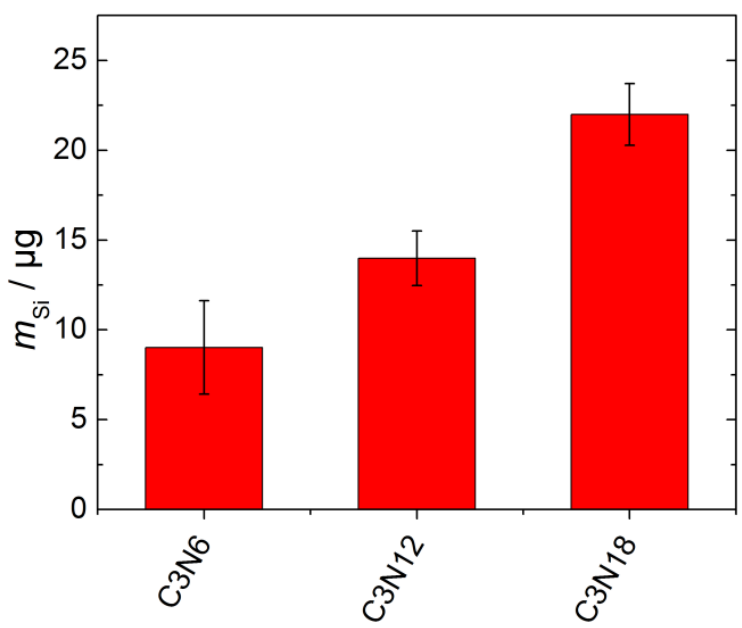

Abbildung 4.1 Überblick über die mittels $\beta$-Molybdatmethode bestimmten Massen $m_{\mathrm{Si}}$ an präzipitiertem Silicium für die Polyamine C3N6, C3N12 und C3N18 mit $\sigma$ Standardabweichung und $n$ Anzahl der Messungen. Reaktionsbedingungen: $30 \mathrm{mM}$ Phosphatpuffer $\mathrm{pH} 6,8, c_{\mathrm{N}}=1 \mathrm{mM}, c_{(\mathrm{SiOH})_{4}}=100 \mathrm{mM}$, Reaktionszeit: $10 \mathrm{~min}$.

Obwohl in jeder Probe insgesamt gleich viele Aminogruppen, die für die Induktion der Präzipitation verantwortlich sind, vorhanden sind, zeigt sich ein deutlicher Unterschied im Präzipitationspotenzial in Abhängigkeit der Kettenlänge. Nachdem diese starken Unterschiede zwischen den Polyaminen C3N6, C3N12 und C3N18 offenbar wurden, sollte geklärt werden, inwieweit auch die Morphologie des gebildeten Silica durch die Kettenlänge beeinflusst wird. 


\section{Silicapräzipitationen mit C3N6}

Mit C3N6 erhaltene Präparate wurden zunächst im Rasterelektronenmikroskop (SEM) untersucht, Abbildung 4.2 gibt einen repräsentativen Überblick. Es sind Aufnahmen von mehreren Proben sowie unterschiedliche Vergrößerungen dargestellt. Über weite Bereiche zeigt sich eine einheitliche Verteilung von kugelförmigen Silicapartikeln, deren Größe sich in einem schmalen Bereich bewegt (A-C). Die Oberfläche der Kugeln erscheint glatt und gleichmäßig. In den Bildern D und E ist zusätzlich zu größeren Partikeln eine Population kleinerer Partikel (siehe Vergrößerung in E) sowie unstrukturiertes Material erkennbar. Um genauere Aussagen treffen zu können, wurden die Durchmesser der Objekte bestimmt. Hierzu wurden mehrere Aufnahmen von unterschiedlichen Proben ausgewertet. Die Durchmesser der Kugeln wurden ausgemessen und die erhaltenen Werte wurden in einem Histogramm (Abbildung 4.2 F) aufgetragen. Anschließend wurden an diese Daten zwei Normalverteilungen angepasst. Für C3N6 ergaben sich mit $d_{1}=(27 \pm 5) \mathrm{nm}$ und $d_{2}=(130 \pm 48) \mathrm{nm}$ zwei Durchmesser, wobei $d_{2}$ häufiger auftritt und $d_{1}$ den kleinen Objekten aus Aufnahmen D und E (Vergrößerung) zugeordnet werden kann.
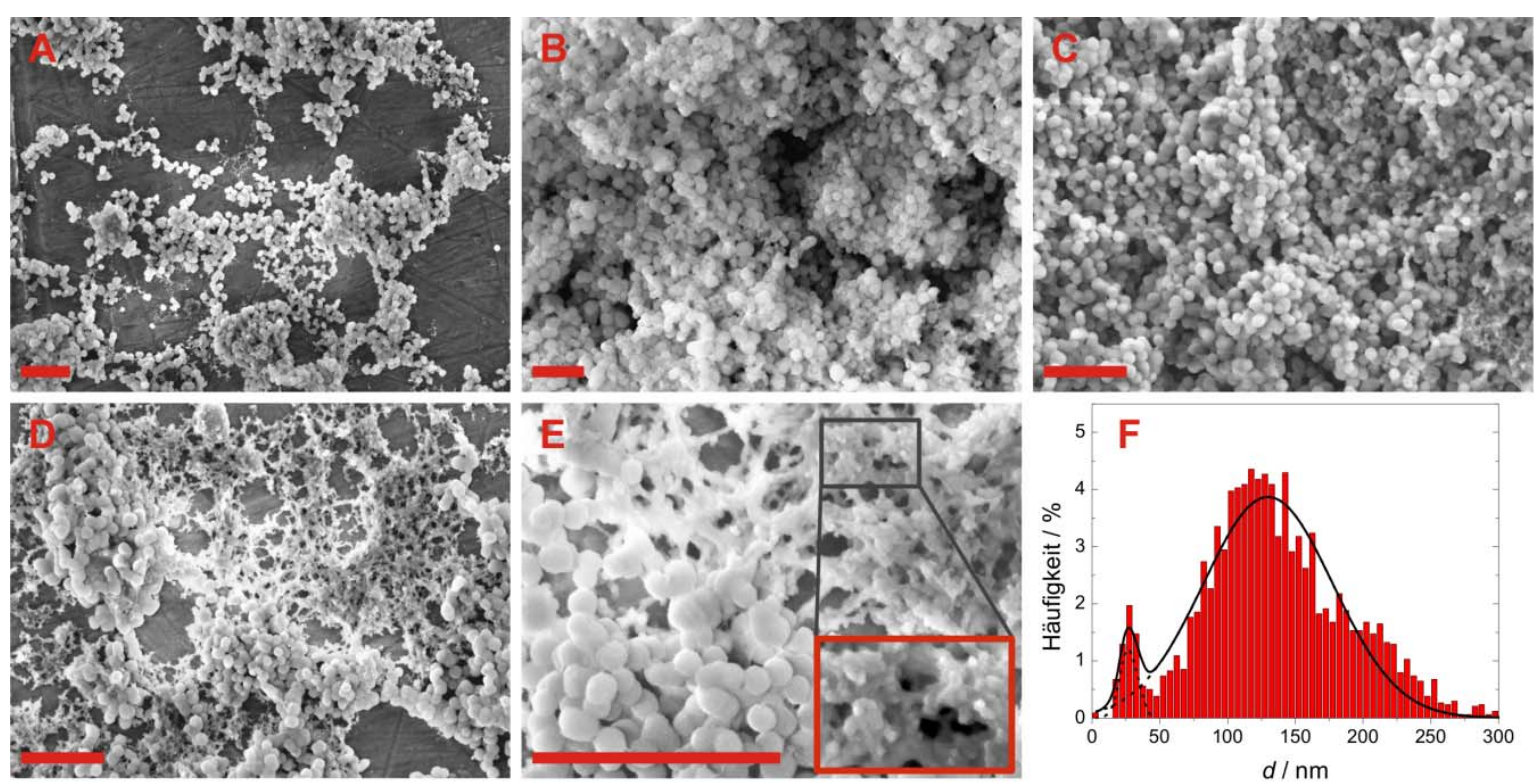

Abbildung 4.2 A-E: Repräsentative SEM-Aufnahmen von Silicapräzipitaten, entstanden durch Zugabe von $\mathrm{Si}(\mathrm{OH})_{4}$ zu Lösungen von C3N6. Maßstabsbalken: $1 \mu \mathrm{m}$. Der rot umrahmte Bereich in $\mathbf{E}$ stellt eine zweifache Vergrößerung des schwarz begrenzten dar. F: Histogramm der auftretenden Kugeldurchmesser $d$ [3398 Kugeln, Binbreite: $5 \mathrm{~nm}$ ]. Die gestrichelten schwarzen Linien geben Anpassungen von Normalverteilungen an die Daten wieder, die darüberliegende durchgezogene Linie stellt die Summe aus den beiden Verteilungen dar. Der Mittelwert und die Standardabweichung der ersten Verteilung liegen bei $d_{l}=(27 \pm 5) \mathrm{nm}$. Der Mittelwert der zweiten Verteilung wurde $\mathrm{zu} d_{2}=(130 \pm 48) \mathrm{nm}$ bestimmt. Reaktionsbedingungen: $30 \mathrm{mM}$ Phosphatpuffer $\mathrm{pH} 6,8, c_{\mathrm{N}}=1 \mathrm{mM}, c_{(\mathrm{SiOH})_{4}}=100 \mathrm{mM}$, Reaktionszeit: $10 \mathrm{~min}$. 
Das entstandene Silica wurde weiterhin im Transmissionselektronenmikroskop (TEM) analysiert, die zugehörigen Aufnahmen sind in Abbildung 4.3 dargestellt. Wie in den SEM-Bildern sind runde Objekte zu finden. Das Signal der Kugeln in den Bildern B und C ist gleichmäßig verteilt, es nimmt zur Objektmitte hin zu, da dort die Elektronen mehr Material durchdringen müssen. Diese Aufnahmen lassen für sich betrachtet auf massive Kugeln schließen. In A und D-F sind jedoch neben diesen Kugeln auch solche erkennbar, bei denen das Signal in der Mitte deutlich schwächer ist. Teilweise treten definierte, deutlich begrenzte Außenschalen auf (A), meistens jedoch werden die Kugeln zur Mitte hin kontinuierlich heller (E). Die Dicke dieser Schalen wurde vermessen und beträgt mit 20-60 nm zwischen $25 \%$ und $35 \%$ des Durchmessers der Kugeln. Im Mittel ergab sich ein Wert von $(32 \pm 2) \%(n=14)$.
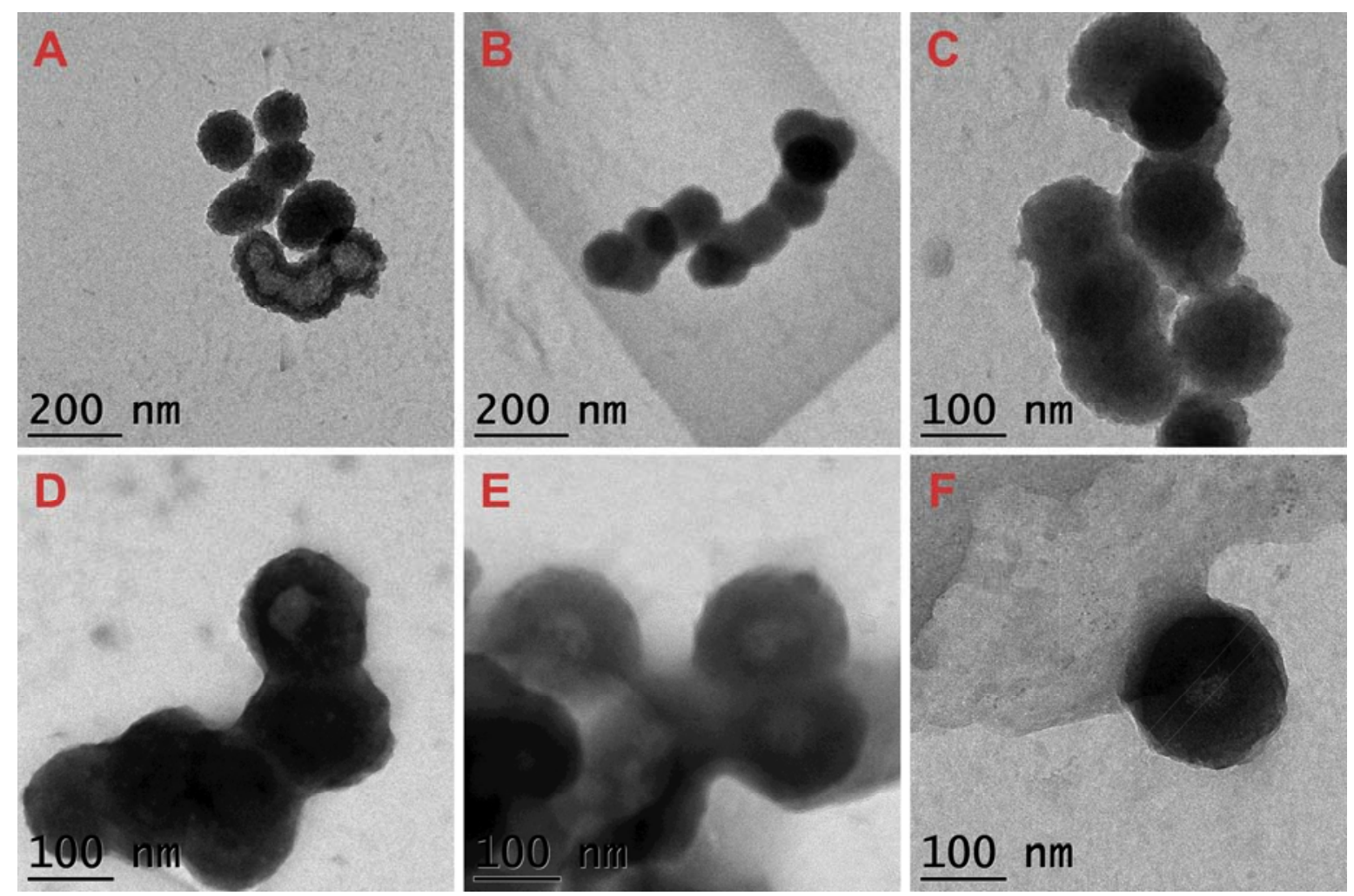

Abbildung 4.3 TEM-Aufnahmen einer Silicaprobe, entstanden durch eine Präzipitationsreaktion mit dem Polyamin C3N6. Reaktionsbedingungen: $30 \mathrm{mM}$ Phosphatpuffer $\mathrm{pH} 6,8, c_{\mathrm{N}}=1 \mathrm{mM}, c_{(\mathrm{SiOH})_{4}}=100 \mathrm{mM}$, Reaktionszeit: $10 \mathrm{~min}$.

Um den tatsächlichen Aufbau der Silicakugeln zu klären, wurden mittels energiedispersiver Röntgenspektroskopie (EDX) die Konzentration und die Verteilung der Elemente Silicium, Sauerstoff und Kohlenstoff in den Proben quantitativ untersucht. Hierzu wird die Probe, anders als bei den oben gezeigten TEM-Messungen, abgerastert (STEM). Zunächst wurde ein STEM-Bild vermessen (Abbildung 4.4 A) und im Anschluss entlang einer Linie 
die Konzentration der Elemente $\mathrm{C}$ (grün), Si (rot) und $\mathrm{O}$ (blau) über emittierte Röntgenstrahlung bestimmt (Abbildung 4.4 B). Dabei wurde davon ausgegangen, dass diese drei Elemente in den Präzipitaten enthalten sind, da Kröger et al. feststellten, dass $\mathrm{SiO}_{2}$ und Polyamine kopräzipitieren und ein Kompositmaterial bilden, aus dem die organische Komponente durch Waschen mit Wasser nicht herausgelöst wird. ${ }^{50,54}$ Die Verteilung von $\mathrm{Si}$ beziehungsweise $\mathrm{O}$ gibt somit die Position von $\mathrm{SiO}_{2}$ wieder, während das C-Signal die Lokalisation von Polyaminen, die aus Kohlen-, Stick- und Wasserstoff aufgebaut sind, erlaubt. Diese Hypothese ist konsistent mit der Beobachtung, dass die Siund O-Verteilungen immer identisch verlaufen. Die relativen Signale dieser beider Elemente, die nicht abgebildet sind, zeigen weiter, dass das O-Signal doppelt so groß ist wie das Si-Signal.
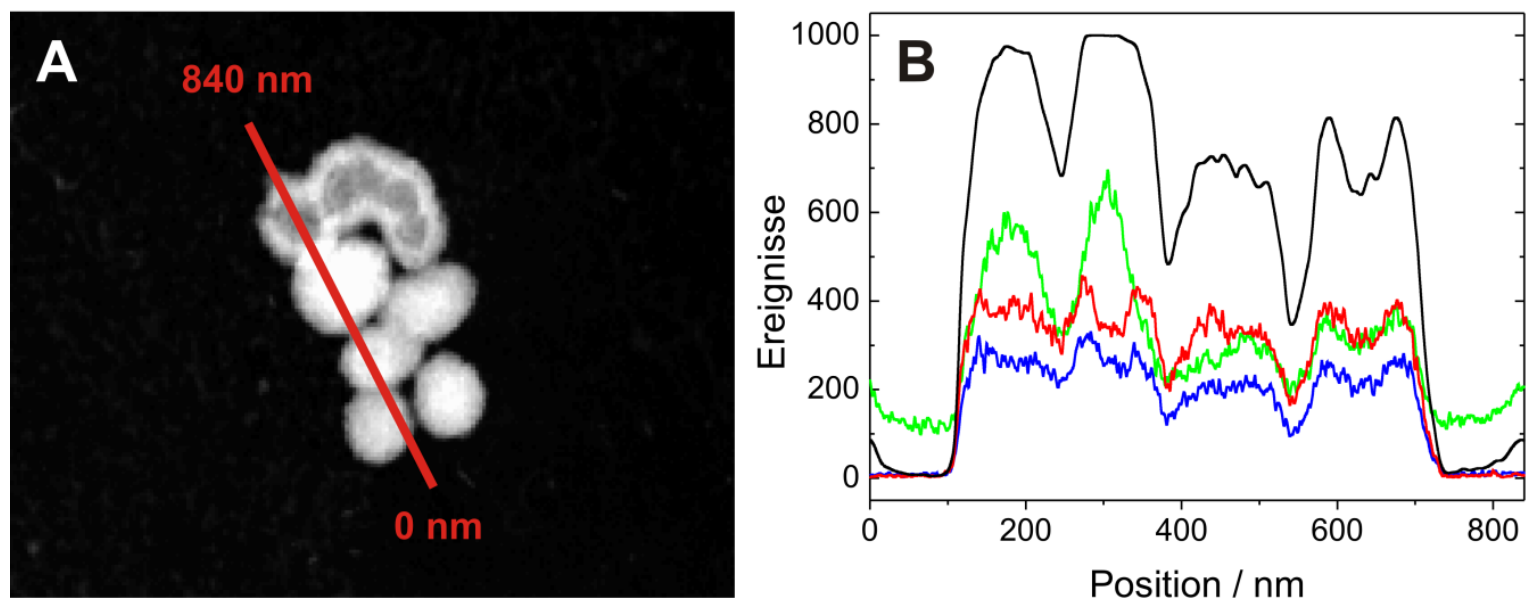

Abbildung 4.4 A STEM-Aufnahme eines aus der Reaktion von $\mathrm{Si}(\mathrm{OH})_{4}$ mit $\mathrm{C} 3 \mathrm{~N} 6$ entstandenen Silicapräzipitats. Reaktionsbedingungen: $30 \mathrm{mM}$ Phosphatpuffer $\mathrm{pH} 6,8, c_{\mathrm{N}}=1 \mathrm{mM}, c_{(\mathrm{SiOH})_{4}}=100 \mathrm{mM}$, Reaktionszeit: $10 \mathrm{~min}$. B EDX-Analyse entlang der in A rot dargestellten Linie. $-\mathrm{C}-\mathrm{Si}-\mathrm{O}-$ auf 1000 normiertes STEM-Signal.

Zunächst sei darauf hingewiesen, dass auch außerhalb der hellen Bereiche in A ein Kohlenstoffsignal detektiert wird (Abbildung 4.4 B: 0-100 nm und 740-840 nm), da das TEM-grid mit Kohlenstoff benetzt ist. Die schwarze Linie gibt das auf 1000 normierte STEM-Signal wieder. Folgt man den Linien von $0 \mathrm{~nm}$ bis $840 \mathrm{~nm}$, zeigt sich bei ca. $100 \mathrm{~nm}$ ein deutlicher STEM-Signalanstieg verbunden mit erhöhter Konzentration aller analysierten Elemente. Auf dem Bild ist erkennbar, dass zunächst durch eine massiv erscheinende Kugel $(100-250 \mathrm{~nm})$ gemessen wurde. Während ab $140 \mathrm{~nm}$ sowohl das Sauerstoff- als auch das Siliciumsignal zunächst nicht mehr ansteigen, geht das Kohlenstoffsignal weiter nach oben und erreicht bei ca. $175 \mathrm{~nm}$ ein lokales Maximum. Beim Querschnitt durch die zweite Kugel $(250-380 \mathrm{~nm})$ ist dieses Phänomen nochmals 
erkennbar. Hier haben Si und O jeweils im Schalenbereich ein Maximum, während die höchste C-Konzentration in der Kugelmitte vorliegt. Die vierte Kugel (540-740 nm) erscheint im STEM-Bild hohl, was auch das STEM-Signal wiedergibt, das in den Schalenbereichen maximal ist. Bei dieser Kugel sind die Verläufe der C-, Si- und O-Signale nahezu identisch. Die Ergebnisse können so gedeutet werden, dass bei allen Kugeln $\mathrm{SiO}_{2}$ überwiegend in den Schalen angereichert und die Konzentration im Kern deutlich geringer ist, unabhängig davon, ob sie in TEM- oder STEM-Aufnahmen hohl oder massiv erscheinen. Solche Kugeln, die massiv aussehen, sind mit organischem Material, also Polyaminen, gefüllt.

Zusätzlich zu den oben dargestellten EDX-Linienscans wurden EDX-Elementbilder aufgenommen. In Abbildung 4.5 sind Aufnahmen mit verschiedenen Informationen dargestellt. A-C geben die Intensität von Kohlenstoff- (A, grün), Silicium- (B, rot) und Sauerstoffsignal (C, blau) wieder. In D hingegen ist das gesamte STEM-Signal dargestellt.
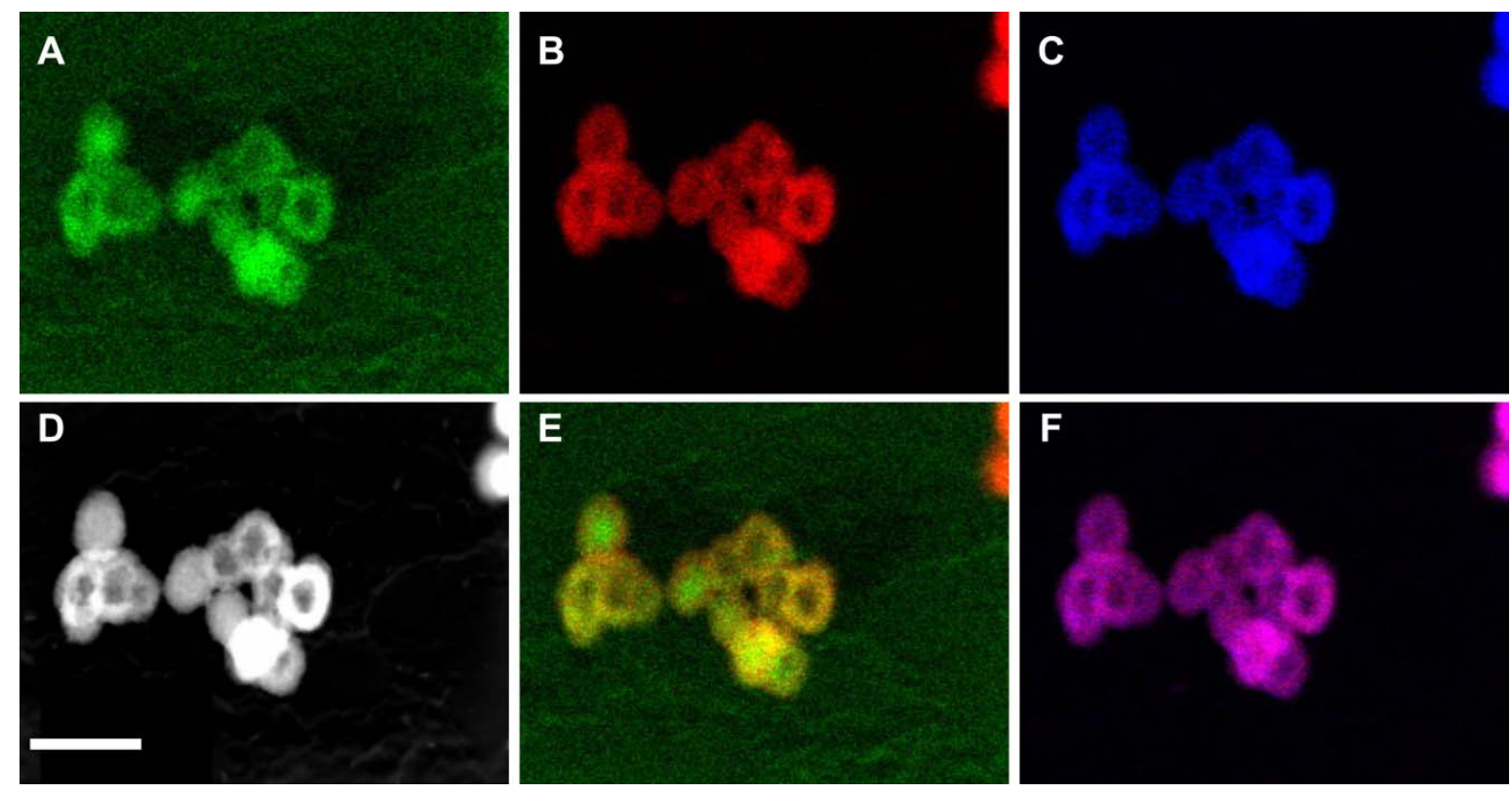

Abbildung 4.5 A-C EDX-Elementbilder der A Kohlenstoff-, B Silicium- und C Sauerstoffintensitäten und D STEM-Signal eines Silicapräzipitats, entstanden durch Zugabe von $\mathrm{Si}(\mathrm{OH})_{4}$ zu einer Lösung von C3N6. Reaktionsbedingungen: $30 \mathrm{mM}$ Phosphatpuffer $\mathrm{pH} 6,8, c_{\mathrm{N}}=1 \mathrm{mM}, c_{(\mathrm{SiOH})_{4}}=100 \mathrm{mM}$, Reaktionszeit: $10 \mathrm{~min}$. E Überlagerung von Abbildungen A und B, F Überlagerung von Abbildungen B und C. Maßstabsbalken: $200 \mathrm{~nm}$.

In E wurden zur Verdeutlichung der vorliegenden Verhältnisse A und B und somit die Kohlenstoff- und Siliciumaufnahmen überlappt. Bei dieser Methode erscheinen Bereiche, in denen ausschließlich Kohlenstoff vorliegt, grün, solche, die nur Silicium aufweisen, rot und bei gleicher Anwesenheit beider Elemente ergibt sich eine gelbe Färbung. In Bild F 
wurden analog Silicium- und Sauerstoffverteilungen überlagert. Hierbei ergibt sich als Mischfarbe bei gleicher Intensität beider Elemente rosa.

Es können ähnliche Schlussfolgerungen gezogen werden wie bei den Linienscans. Das Kohlenstoffbild A gleicht dem des STEM-Signals (E), und zeigt kugelartige Strukturen, von denen einige hohl, andere massiv wirken. Sauerstoff (C) und Silicium (B) hingegen sind nahezu ausschließlich in den äußeren Bereichen der Partikel vorhanden und ihre Verteilung ist deckungsgleich (F). Der Vergleich des Kohlenstoffsignals stellvertretend für die Polyaminverteilung mit dem Siliciumsignal von $\mathrm{SiO}_{2}$ verdeutlicht nochmals, dass in den Schalen der Partikel Polyamine und Silica vorhanden sind, die Innenräume jedoch entweder hohl oder mit Polyaminen gefüllt sind. Diese Erkenntnis bestätigt die These, dass bezogen auf Silica keine massiven Kugeln bei der polyamininduzierten Präzipitation von Kieselsäure entstehen. Vielmehr sind in manchen Partikeln Polyamine eingeschlossen, die vermutlich nicht an der Reaktion beteiligt waren.

Zusammenfassend lässt sich feststellen, dass mit C3N6 unter Zugabe von monomerer Kieselsäure bei den gewählten Reaktionsbedingungen um $130 \mathrm{~nm}$ große kugelartige Silicapartikel gebildet werden, deren Größe nicht stark variiert. Sie sind hohl und bestehen aus $\mathrm{SiO}_{2}$ sowie Polyaminen, wobei ihr Innenraum teilweise mit Polyaminen angereichert ist. Die Dicke der Schalen beträgt ungefähr 33 \% des Durchmessers der Kugeln.

\section{Silicapräzipitationen mit C3N12}

Auch Silica, dessen Bildung durch C3N12 induziert wurde, wurde mit verschiedenen elektronenmikroskopischen Techniken charakterisiert. In Abbildung 4.6 sind SEMAufnahmen zusammengestellt, die einen Überblick über das Aussehen der Partikel aus mehreren Reaktionsansätzen geben. Wie auch bei C3N6 (Abbildung 4.2) zeigt sich ein sehr gleichmäßiges Bild, es liegen kugelartige Strukturen vor, die eine glatte Oberfläche aufweisen $(\mathrm{D} / \mathrm{E})$ und die in ihrer Größe kaum variieren (A-C). Eine statistische Analyse des Kugeldurchmessers ist in einem Histogramm (Abbildung 4.6 F) dargestellt. Anders als bei C3N6 tritt nur eine Population auf, die mit $d=(169 \pm 23) \mathrm{nm}$ größere Durchmesser aufweist und deren Verteilung enger ist. 

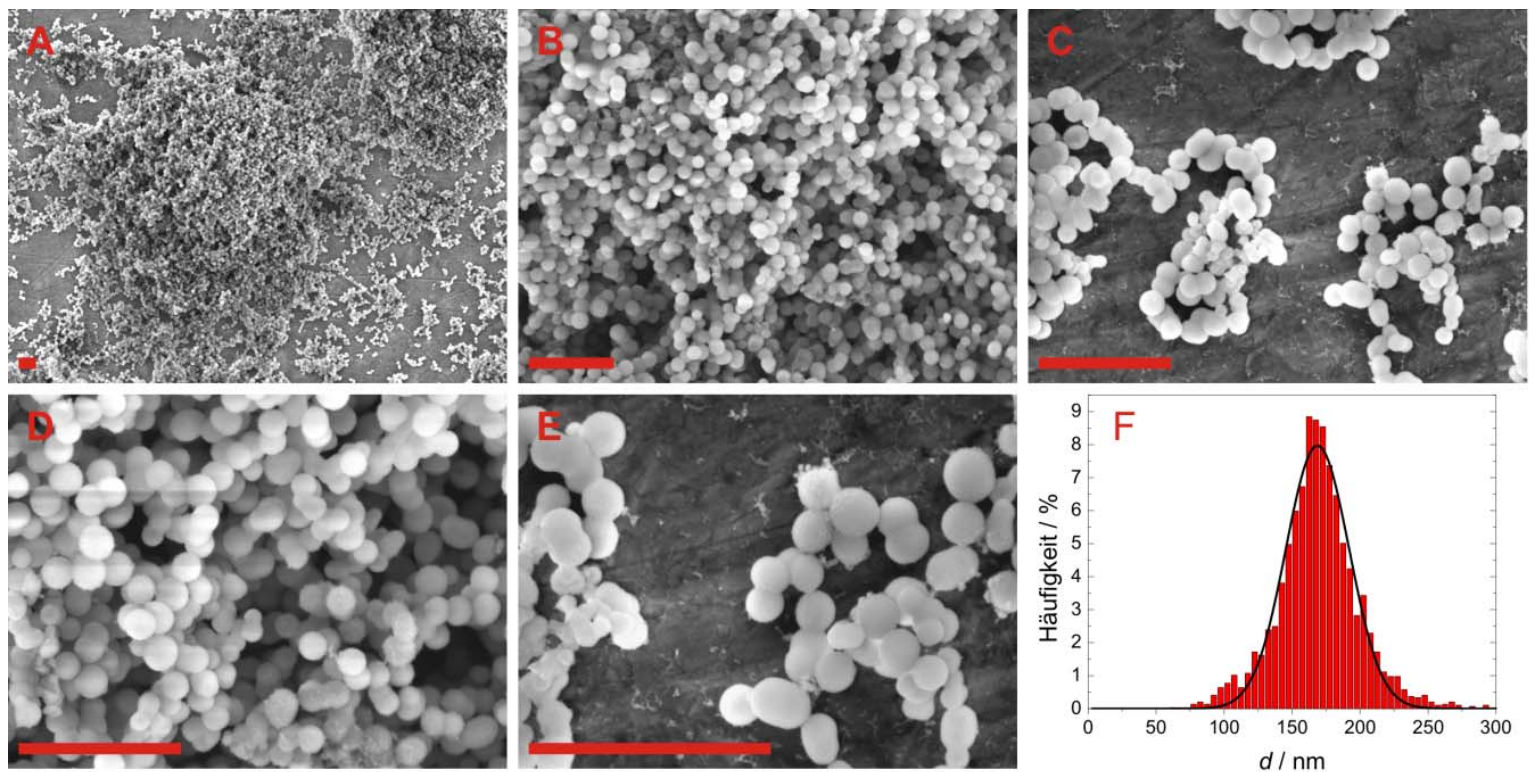

Abbildung 4.6 A-E: SEM-Aufnahmen von Silicapräzipitaten, deren Bildung durch C3N12 induziert wurde. Maßstabsbalken: $1 \mu \mathrm{m}$. F: Histogramm der auftretenden Kugeldurchmesser $d$ [2973 Kugeln, Binbreite: $5 \mathrm{~nm}$ ]. An die Daten wurde eine Gaußverteilung angepasst (schwarze Linie), deren Mittelwert $d=(169 \pm 23) \mathrm{nm}$ beträgt. Reaktionsbedingungen: $30 \mathrm{mM}$ Phosphatpuffer $\mathrm{pH} 6,8, \quad c_{\mathrm{N}}=1 \mathrm{mM}$, $c_{(\mathrm{SiOH})_{4}}=100 \mathrm{mM}$, Reaktionszeit: $10 \mathrm{~min}$.

Transmissionselektronenmikroskopische Messungen (Abbildung 4.7) zeigen ausschließlich Kugeln, die hohl aussehen. Während die Kugeln an ihrer Oberfläche glatt sind, erscheint
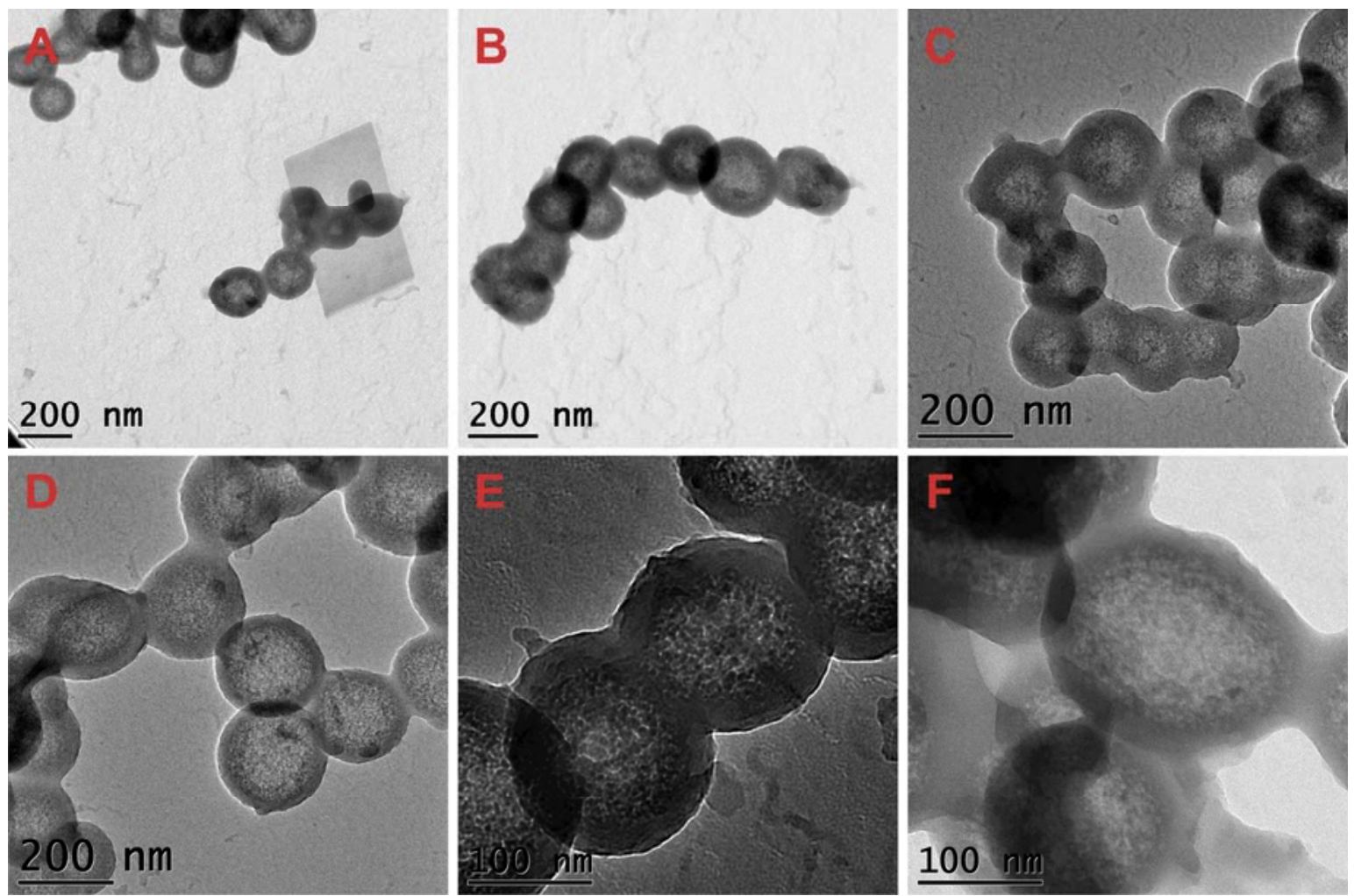

Abbildung 4.7 TEM-Aufnahmen eines Silicapräzipitats, entstanden durch Zugabe von $\mathrm{Si}(\mathrm{OH})_{4}$ zu einer Lösung von C3N12. Reaktionsbedingungen: $30 \mathrm{mM}$ Phosphatpuffer $\mathrm{pH} 6,8, c_{\mathrm{N}}=1 \mathrm{mM}, c_{(\mathrm{SiOH})_{4}}=100 \mathrm{mM}$, Reaktionszeit: $10 \mathrm{~min}$. 
das Innere unregelmäßig. Einige Kugeln scheinen miteinander verwachsen zu sein, wie in Aufnahmen $\mathrm{C}$ und $\mathrm{E}$ zu sehen ist, andere wiederum sind deutlich voneinander separiert. Die Dicke der Schalen, bestimmt aus den TEM- und STEM-Aufnahmen (Abbildung 4.8), beträgt mit 25-50 nm 15-45 \% des Kugeldurchmessers, der Mittelwert liegt bei (24 \pm 4$) \%$ $(n=37)$ und ist damit kleiner als bei C3N6 (33\%).

EDX-Messungen an einer Probe von mit C3N12 präzipitiertem Silica ergeben ähnliche Erkenntnisse wie für C3N6. Bei der Detektion von emittierter Röntgenstrahlung entlang einer $870 \mathrm{~nm}$ langen Linie, die zwei Kugeln durchquert (Abbildung $4.8 \mathrm{~A}$ ), wird deutlich, dass $\mathrm{SiO}_{2}$ im Äußeren der Kugeln deutlich höher konzentriert ist, als in ihrer Mitte. Bei ca. $230 \mathrm{~nm}, 380 \mathrm{~nm}, 450 \mathrm{~nm}$ und $630 \mathrm{~nm}$, also in den Schalen, erreichen sie jeweils maximale Intensität. Die Signale für Si (rote Linie) und O (Sauerstoff) verlaufen dabei fast völlig analog zum normierten STEM-Signal, das in schwarz dargestellt ist (Abbildung 4.8 B). Auffällig ist bei der Messung, dass das Kohlenstoffsignal sehr niedrig ist und sich nur wenig von dem aus der Umgebung der Kugeln abhebt. Dies konnte an mehreren Stellen der Probe beobachtet werden.
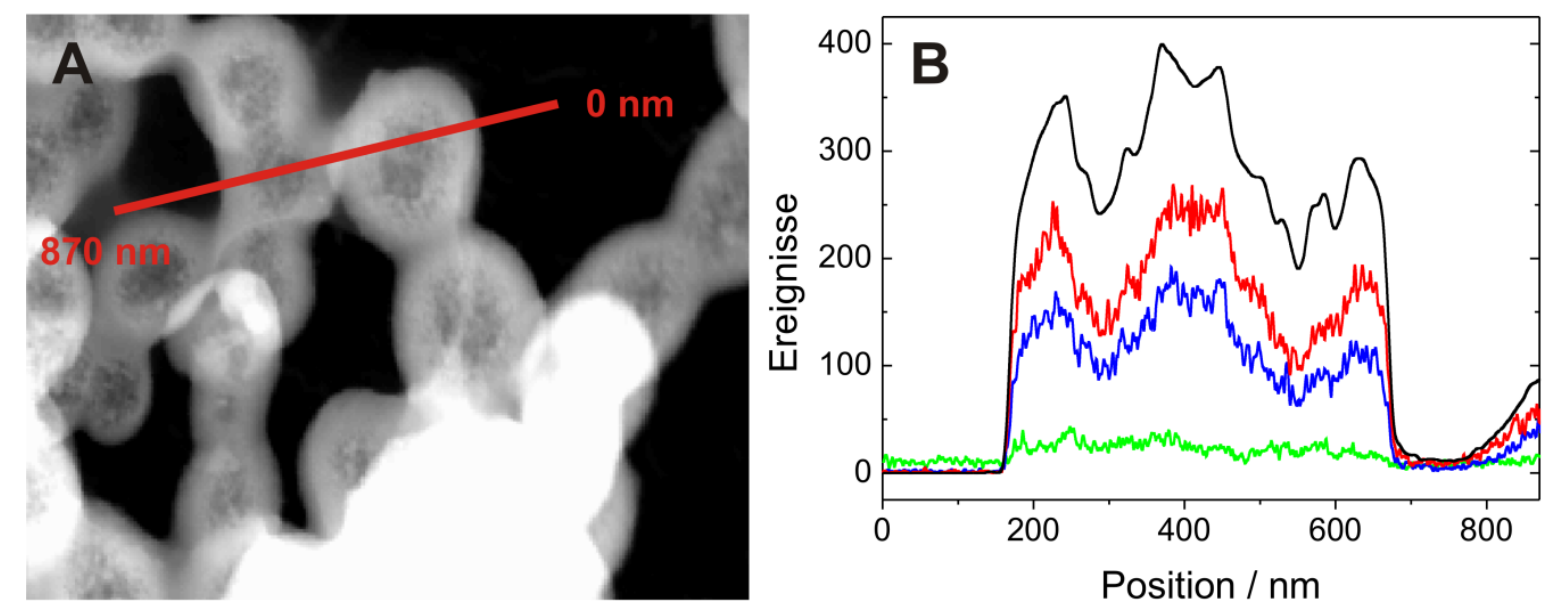

Abbildung 4.8 A STEM-Aufnahme eines mit dem Polyamin C3N12 gebildeten Silicapräzipitats. Reaktionsbedingungen: $30 \mathrm{mM}$ Phosphatpuffer $\mathrm{pH} 6,8, c_{\mathrm{N}}=1 \mathrm{mM}, c_{(\mathrm{SiOH})_{4}}=100 \mathrm{mM}$, Reaktionszeit: $10 \mathrm{~min}$. B EDX-Linienanalyse entlang der in A rot dargestellten Linie. $-\mathrm{C}-\mathrm{Si}-\mathrm{O}-$ auf 400 normiertes STEM-Signal.

Auch in den EDX-Elementbildern (Abbildung 4.9) wurde kein hohes Kohlenstoffsignal (A, grün) detektiert, es hebt sich kaum vom Untergrund ab. Dagegen sind Silicium (B, rot) und Sauerstoff (C, blau) deutlich in den Schalen der Kugeln, die im STEM-Bild (D) zu sehen sind, messbar. Die Signale von Si und O sind dabei, wie schon bei den Messungen mit C3N6 beobachtet wurde, vollkommen deckungsgleich (F). Die Überlagerung von Cund Si-Signal (E) liefert keine weiteren Erkenntnisse zur niedrigen Kohlenstoff- und damit 
vermutlich niedrigen Polyaminkonzentration. Eine Anreicherung von Kohlenstoff im Kugelinneren, wie sie mit C3N6 (Abbildung 4.5) teilweise festgestellt wurde, fehlt völlig, was sich mit der bei den TEM-Messungen gemachten Beobachtung deckt, dass es keinerlei massiv aussehende Kugeln gibt.
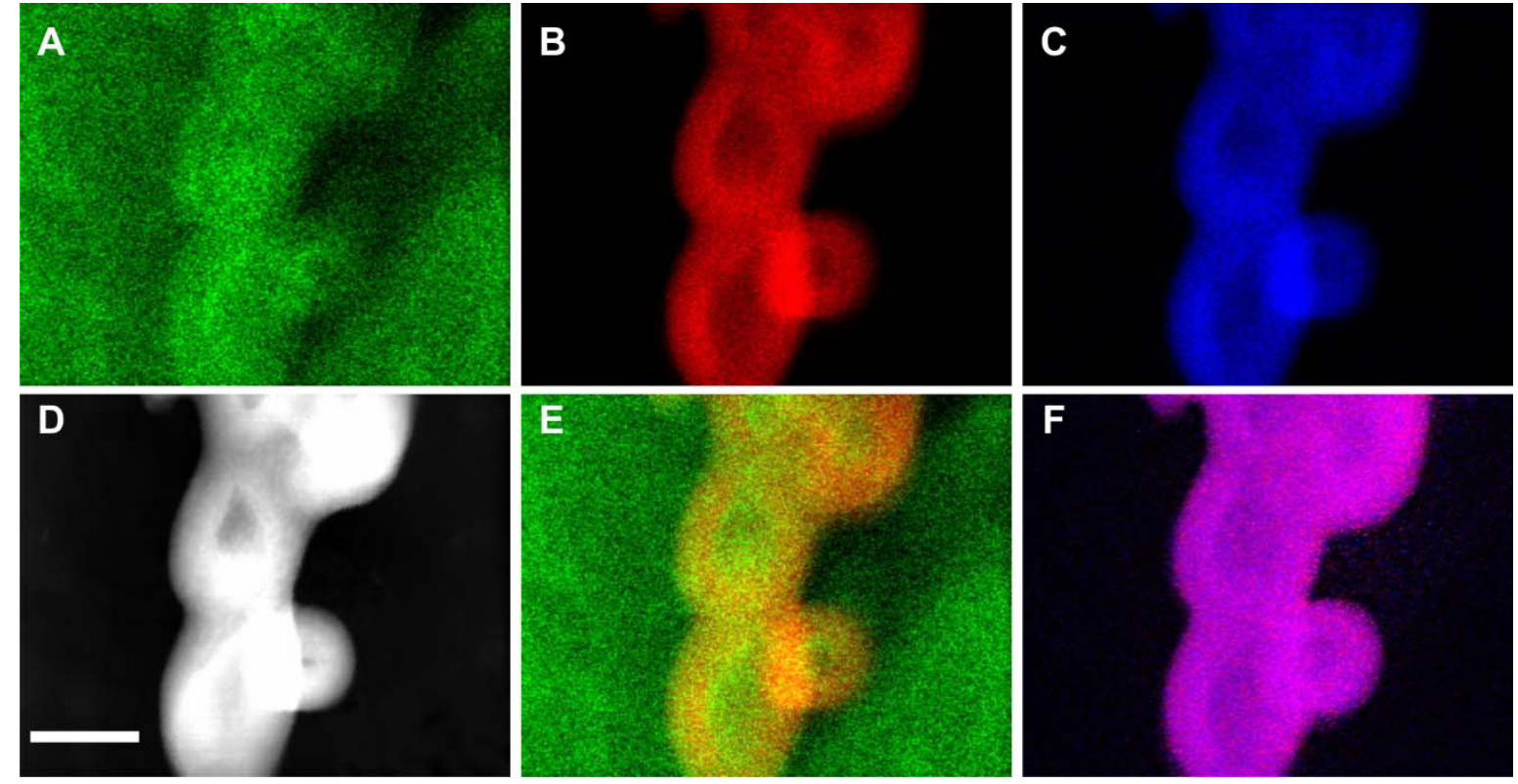

Abbildung 4.9 EDX-Aufnahmen einer Silicaprobe, entstanden aus einer $\mathrm{SiO}_{2}$-Präzipitationsreaktion mit dem Polyamin C3N12. A-C EDX-Elementbilder der A Kohlenstoff-, B Silicium- und C Sauerstoffintensitäten und D STEM-Signal. Reaktionsbedingungen: $30 \mathrm{mM}$ Phosphatpuffer $\mathrm{pH} 6,8, c_{\mathrm{N}}=1 \mathrm{mM}, c_{(\mathrm{SiOH})_{4}}=100 \mathrm{mM}$, Reaktionszeit: 10 min. E Überlagerung von Abbildungen A und B, F Überlagerung von Abbildungen $\mathrm{B}$ und $\mathrm{C}$. Maßstabsbalken: $100 \mathrm{~nm}$.

Bei der durch das Polyamin C3N12 induzierten Präzipitation von Kieselsäure werden, wie bei C3N6, Kugeln gebildet. Ihre Größenverteilung ist enger, im Mittel liegt der Durchmesser bei $169 \mathrm{~nm}$. Die Schalen der Kugeln bestehen wiederum aus $\mathrm{SiO}_{2}$ und Polyaminen, wobei der Polyaminanteil sehr niedrig zu sein scheint. Die Kugeln sind ausschließlich hohl und nicht mit organischem Material gefüllt. Die Schalendicke ist mit $24 \%$ des Kugeldurchmessers kleiner als dies bei C3N6 der Fall ist.

\section{Silicapräzipitationen mit C3N18}

Bei der Zugabe von Kieselsäure zu Lösungen von C3N18 in Phosphatpuffer entsteht wie auch mit C3N6 und C3N12 Silica, wobei die quantitativen Messungen gezeigt haben, dass mit C3N18 die größte Menge gebildet wird (Abbildung 4.1). Um eine Erklärung hierfür zu finden, wurden auch von diesen Proben elektronenmikroskopische Aufnahmen gemacht. In den SEM-Aufnahmen aus Abbildung 4.10 zeigen sich anders als bei den Experimenten mit 
C3N6 und C3N12 nicht ausschließlich kugelartige Strukturen. Während in A und G gleichmäßig große Kugeln glatter Beschaffenheit zu sehen sind, finden sich weiterhin filamentöse Strukturen (B-F) sowie Zwischenstufen von beiden (D, Vergrößerung). In C und $\mathrm{F}$ ist viel Material erkennbar, das wirkt, als wäre seine Struktur durch den Zerfall von Fasern entstanden. Die langen Strukturen, die an manchen Stellen aussehen, als wären sie flache Bänder (E, Vergrößerung und Pfeile) sind teilweise zerbrochen (F).
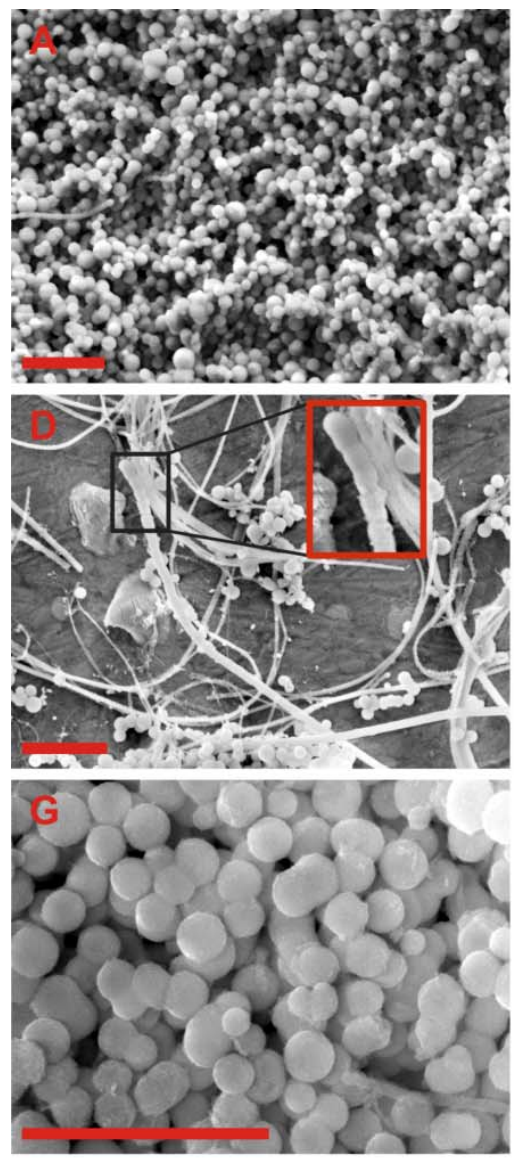
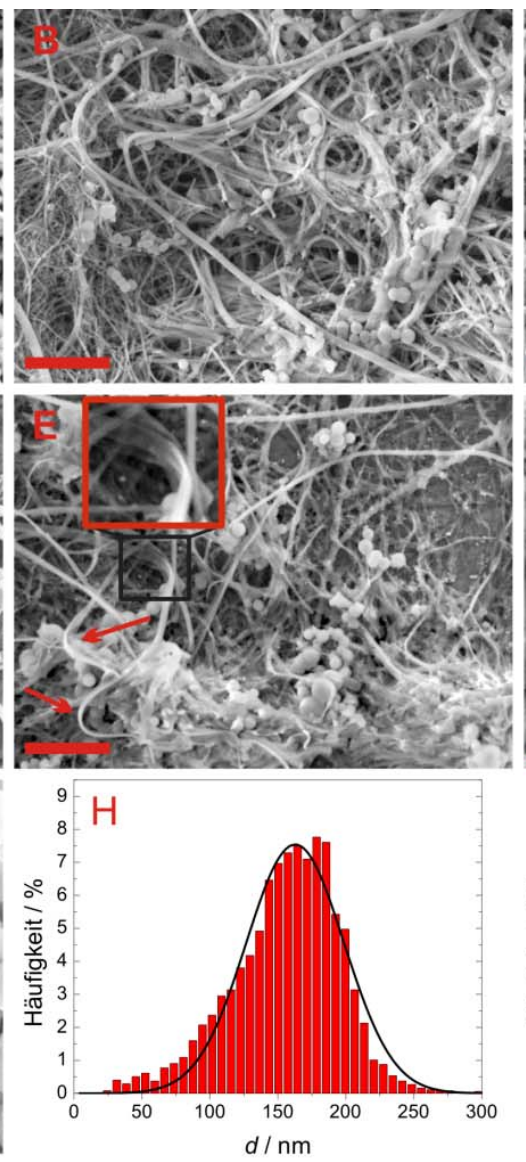

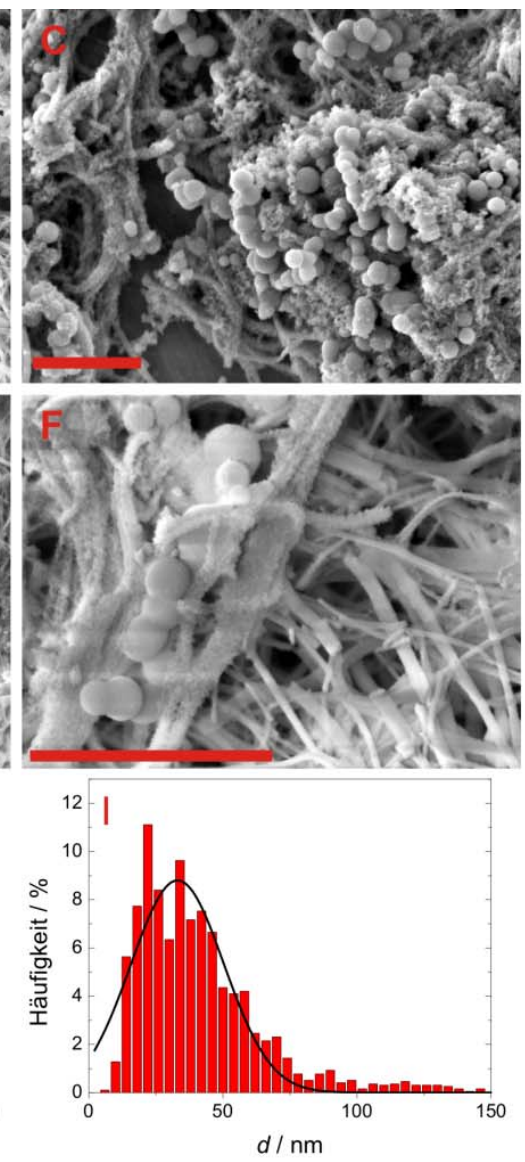

Abbildung 4.10 A-G: SEM-Messungen: Aufnahmen von Silica, das bei Präzipitationen mit C3N18 entstand. In $\mathrm{D}$ und $\mathrm{E}$ stellt jeweils der rot umrahmte Bereich die zweifache Vergrößerung des schwarz umrahmten Rechtecks dar. Maßstabsbalken: $1 \mu \mathrm{m}$. H: Auftragung der Kugeldurchmesser $d$ in einem Histogramm [3759 Kugeln, Binbreite: $7 \mathrm{~nm}$ ] und zugehörige angepasste Gaußverteilung. Die Größe $d$ der Kugeln beträgt im Mittel $(163 \pm 36) \mathrm{nm}$. I: Histogramm der auftretenden Filamentdurchmesser $d_{F}$ [1953 Filamente, Binbreite: $4 \mathrm{~nm}$ ] sowie Anpassung einer Gaußverteilung an die Daten (schwarze Linie). Der Mittelwert mit Standardabweichung wurde $\mathrm{zu} \quad d_{F}=(33 \pm 17) \mathrm{nm}$ bestimmt. Reaktionsbedingungen: $30 \mathrm{mM}$ Phosphatpuffer $\mathrm{pH} 6,8, c_{\mathrm{N}}=1 \mathrm{mM}, c_{(\mathrm{SiOH})_{4}}=100 \mathrm{mM}$, Reaktionszeit: $10 \mathrm{~min}$.

Zur Beschreibung der Morphologie wurden bei diesen Proben die Durchmesser der auftretenden Kugeln (Abbildung $4.10 \mathrm{H}$ ) ermittelt. An die Daten aus dem Histogramm wurde eine Normalverteilung angepasst, die ein mittleren Durchmesser von $d=(163 \pm 36) \mathrm{nm}$ liefert. Die Länge der Filamente konnte nicht bestimmt werden, da diese teilweise über einzelne Bilder hinaus verlaufen, sie sind in vielen Fällen über $10 \mu \mathrm{m}$ lang. 
Die Auswertung ihrer Durchmesser (Abbildung 4.10 I; Anzahl Bänder: 1953, Binbreite: $4 \mathrm{~nm})$ ergab einen Mittelwert von $d_{F}=(33 \pm 17) \mathrm{nm}$.

Bei transmissionselektronenmikroskopischen Untersuchungen von mit C3N18 gebildetem Silica konnten die oben beschriebenen Strukturen wiedergefunden werden. Neben kugelförmigem Material liegen auch Filamente vor, außerdem ist eine längliche Struktur erkennbar, die eine Zwischenstufe zwischen beiden darstellt (Abbildung 4.11). Die auftretenden Kugeln sind wie mit $\mathrm{C} 3 \mathrm{~N} 12$ an der Oberfläche ebenmäßig, ihr Inneres dagegen wirkt heterogen $(\mathrm{C})$. Die Dicke ihrer Schalen wurde vermessen und liegt zwischen $20 \mathrm{~nm}$ und $50 \mathrm{~nm}$ und somit $15-35 \%$ des Kugeldurchmessers. Im Mittel beträgt sie $(23 \pm 3) \%(n=20)$. In Aufnahme B, die an der gleichen Stelle wie Aufnahme A jedoch zu einem späteren Zeitpunkt gemacht wurde, ist ein dunkler Hintergrund erkennbar und es erscheinen manche Kugeln größer als in A. Dies ist auf eine Verunreinigung mit Kohlenstoff zurückzuführen, die teilweise bei den Messungen auftritt.
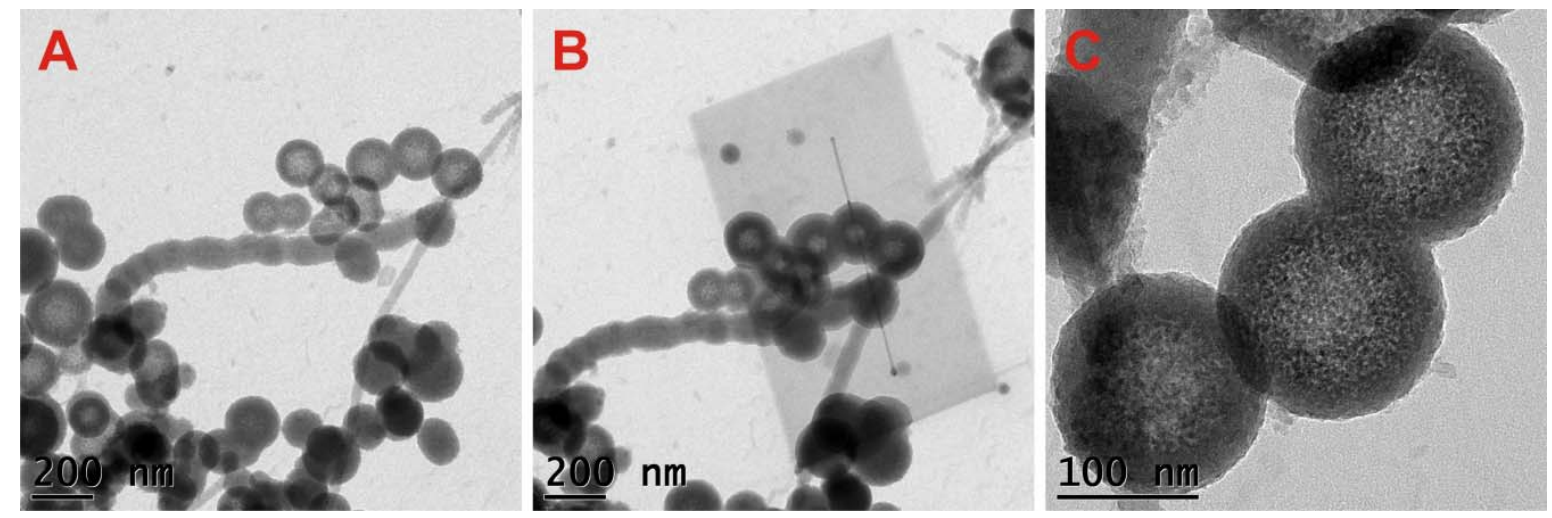

Abbildung 4.11 TEM-Aufnahmen eines Silicapräzipitats, gebildet nach Zugabe von $\mathrm{Si}(\mathrm{OH})_{4} \mathrm{zu}$ dem Polyamin C3N18. Reaktionsbedingungen: $30 \mathrm{mM}$ Phosphatpuffer $\mathrm{pH} \mathrm{6,8,} c_{\mathrm{N}}=1 \mathrm{mM}, c_{(\mathrm{SiOH})_{4}}=100 \mathrm{mM}$, Reaktionszeit: 10 min.

Auch bei den EDX-Linienanalysen wirkt sich die Verunreinigung mit Kohlenstoff aus (Abbildung 4.12). In Graph B ist erkennbar, dass das C- (grün) und das auf 200 normierte STEM-Signal (schwarz) einen größeren Bereich umfassen, als dies bei Si (rot) und O (blau) der Fall ist. Es ist davon auszugehen, dass dadurch auch die Kohlenstoffkonzentration zu hoch wiedergegeben wird, was Aussagen bezüglich dieser schwierig macht. Eindeutig zeichnet sich jedoch ab, dass bei der zweiten gemessenen Kugel (340$490 \mathrm{~nm}$ ) im Innenraum die Silicium- und Sauerstoffkonzentration niedriger ist, als im Randbereich. Die erste Kugel (140-280 nm) überschneidet genau in ihrer Mitte ein Filament. Die erhöhte Intensität von Si und O im Bereich des Innenraums dieser Kugel 
kann unter der Annahme, dass die Filamente ebenso wie die Kugelschalen aus $\mathrm{SiO}_{2}$ bestehen und die Signale beider Strukturen sich addieren, erklärt werden.
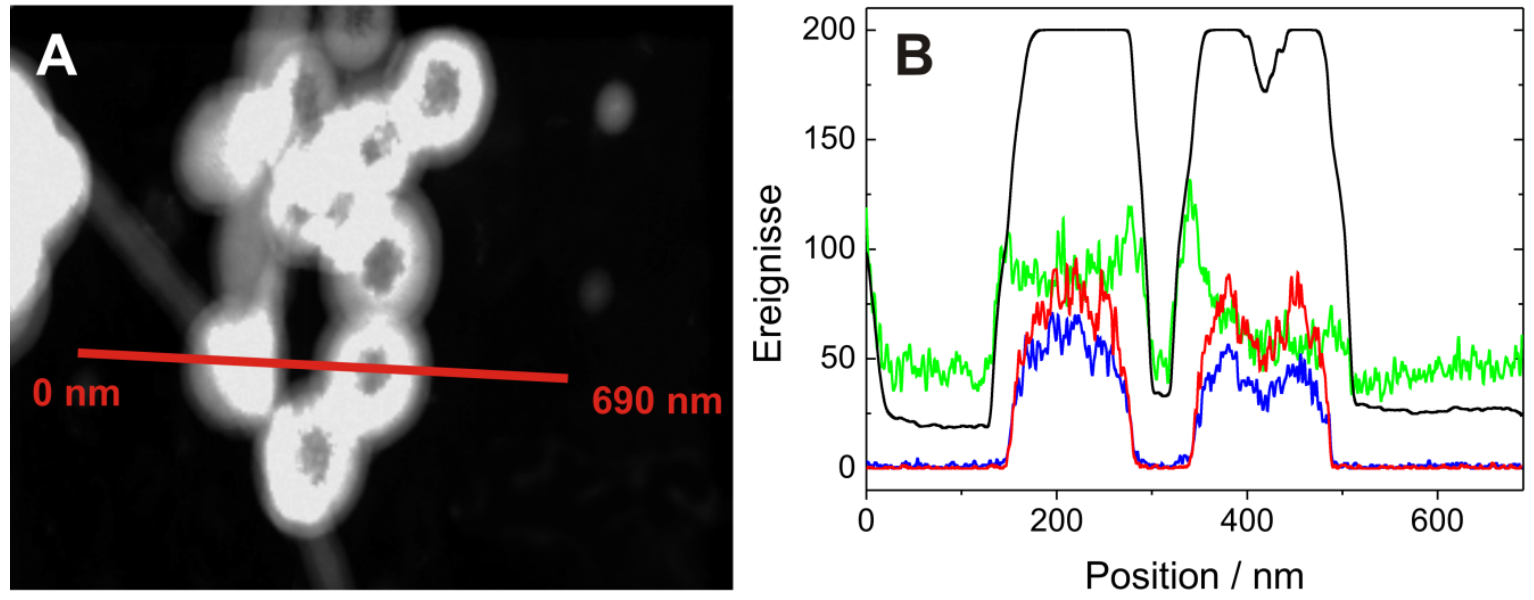

Abbildung 4.12 A STEM-Aufnahme von Silica, das bei der Reaktion von $\mathrm{Si}(\mathrm{OH})_{4}$ mit $\mathrm{C} 3 \mathrm{~N} 18$ entstand. Reaktionsbedingungen: $30 \mathrm{~mm}$ Phosphatpuffer $\mathrm{pH} 6,8, c_{\mathrm{N}}=1 \mathrm{mM}, c_{(\mathrm{SiOH})_{4}}=100 \mathrm{mM}$, Reaktionszeit: $10 \mathrm{~min}$. B EDX-Analyse entlang der in A rot dargestellten Linie. $-\mathrm{C}-\mathrm{Si}-\mathrm{O}-$ auf 200 normiertes STEMSignal.

In EDX-Elementbildern (Abbildung 4.13) zeichnet sich ein ähnliches Bild ab. Die Kohlenstoffverteilung (A) und das STEM-Bild (D) suggerieren durch die Verunreinigungen einen größeren Kugeldurchmesser als die Silicium- und Sauerstoffverteilungen.
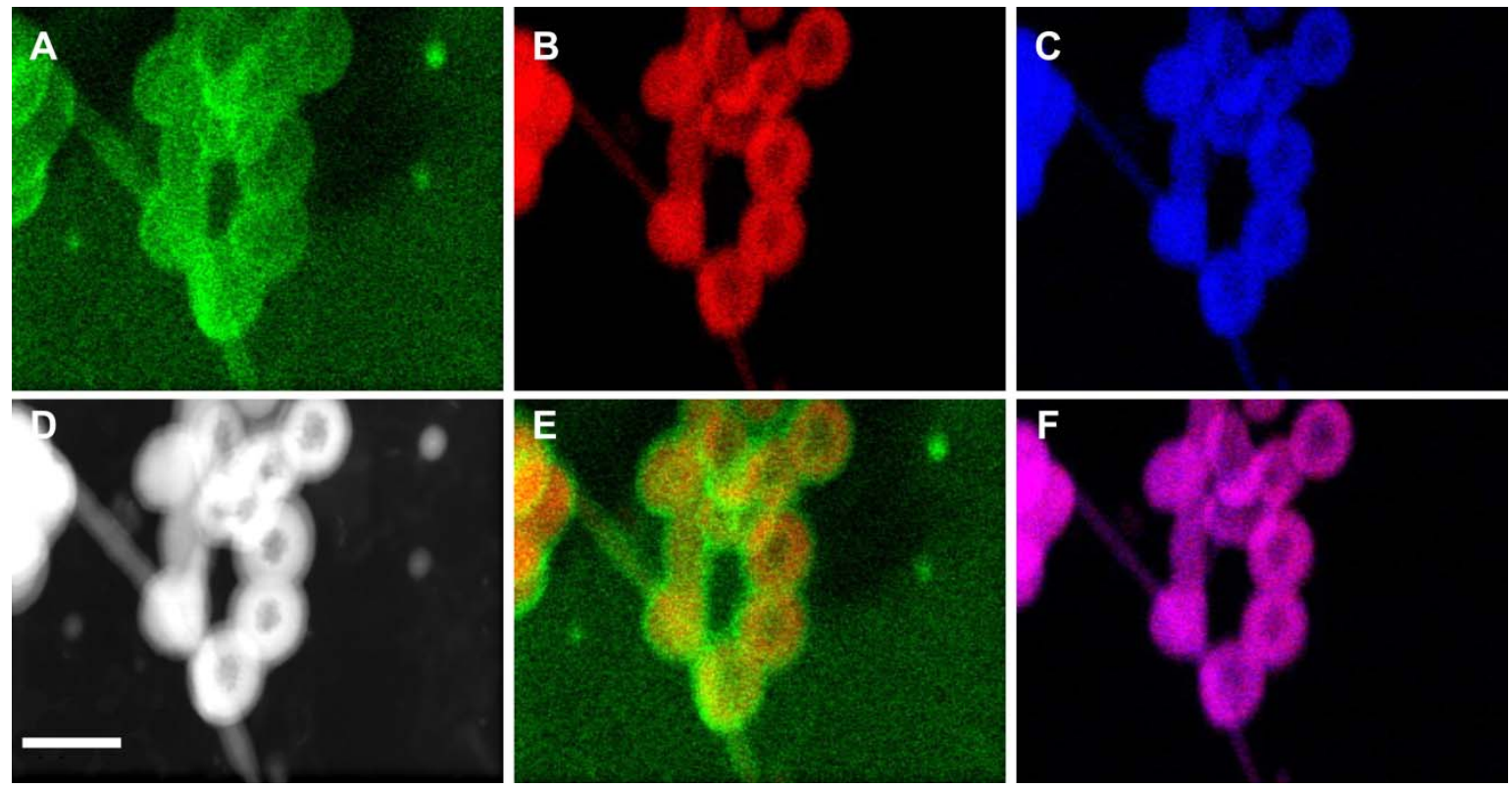

Abbildung 4.13 A-C EDX-Elementbilder der A Kohlenstoff-, B Silicium- und C Sauerstoffintensitäten und D STEM-Signal eines C3N18-Silicapräzipitats. Reaktionsbedingungen: $30 \mathrm{mM}$ Phosphatpuffer pH 6,8, $c_{\mathrm{N}}=1 \mathrm{mM}, c_{(\mathrm{SiOH})_{4}}=100 \mathrm{mM}$, Reaktionszeit: $10 \mathrm{~min}$. E Überlagerung der Abbildungen A und B, F Überlagerung der Abbildungen B und C. Maßstabsbalken: $200 \mathrm{~nm}$. 
Deutlich erkennbar ist das Phänomen in der Überlagerung der C- und Si-Aufnahme (E). Die Hypothese, die anhand der Linienanalyse aufgestellt wurde, die Filamente wären auch aus $\mathrm{SiO}_{2}$ aufgebaut, kann hier bestätigt werden. In $\mathrm{B}$ und $\mathrm{C}$, aber besonders deutlich in deren Überlagerung (F), sind eindeutige Si- und O-Signale im Bereich der Faser sichtbar. Die polyamininduzierte Silicapräzipitation führt mit allen verwendeten Substanzen zur Bildung von hohlen $\mathrm{SiO}_{2}$-Kugeln, die im Fall von C3N18 mittlere Durchmesser von $163 \mathrm{~nm}$ besitzen. Mit diesem längsten Polyamin entstehen nicht nur die größten Mengen an Silica, es zeigen sich zusätzlich filamentartige Strukturen. Es wurde nachgewiesen, dass diese ebenfalls aus $\mathrm{SiO}_{2}$ bestehen. Wie bei C3N12 konnten keine gefüllten Kugeln detektiert werden.

\section{Zusammenfassung}

Die Propylpolyamine unterschiedlicher Kettenlänge (C3N6, C3N12 und C3N18) präzipitieren ungleiche Silicamengen und auch die Morphologie der Präzipitate unterscheidet sich zum einen in den auftretenden Formen, zum anderen in den Partikelgrößen. Um zu testen, ob sich die Verteilungen der Kugeln signifikant unterscheiden, wurden die Daten mit dem Mann-Whitney-U-Test analysiert. ${ }^{88,90}$ Die Partikeldurchmesser aus den Proben sind demnach mit hoher Signifikanz $(P<0,001)$ für alle drei Polyamine voneinander unterschiedlich.

\section{Variation der Polyamin- und $\mathrm{Si}(\mathrm{OH})_{4}$-Konzentrationen bei Präzipitationen}

Es ist bekannt, dass bei der Silicabildung die Konzentration der präzipitationsinduzierenden Komponenten, wie Silaffine oder Polyamine, die Masse an entstehendem $\mathrm{SiO}_{2}$ beeinflusst. Um diesen Effekt zu quantifizieren, wurden Lösungen mit auf Stickstoff bezogenen Konzentrationen von $1 \mathrm{mM}$ und $2 \mathrm{mM}$ miteinander verglichen. Die Konzentration an $\mathrm{Si}(\mathrm{OH})_{4}$ betrug in allen Ansätzen 100 mM. In Abbildung 4.14 sind die Daten dieser Untersuchungen zusammengefasst. Im Fall von C3N6 erhöht sich die Menge an präzipitiertem $\mathrm{SiO}_{2}$ bei Verdoppelung der Polyaminkonzentration um das ca. 3,5fache auf 31,0 $\mu$ g. Eine etwa 2,5fach größere Menge ergab sich mit C3N12 und C3N18 bei Verdopplung der auf Stickstoff bezogenen Polyaminkonzentration.

In diesem Kontext wurde stellvertretend mit C3N12 untersucht, wie sich eine veränderte Kieselsäurekonzentration auf die Präzipitationsmenge auswirkt. In vielen Arbeiten zu 
biomimetischen Silicapräzipitationen wird mit einer $\mathrm{Si}(\mathrm{OH})_{4}$-Konzentration von $40 \mathrm{~mm}$ gearbeitet, weshalb diese Konzentration verwendet wurde. Dabei entstand die gleiche Menge an Silica, wie bei Verwendung von $100 \mathrm{mM} \mathrm{Si}(\mathrm{OH})_{4}$. Bei den vorliegenden Reaktionsbedingungen wird demnach mit einem Überschuss von $\mathrm{Si}(\mathrm{OH})_{4}$ gearbeitet, der die Präzipitation nicht beeinflusst, während die Polyaminkonzentration sich deutlich auswirkt.

\begin{tabular}{lcccc}
\hline & $c_{\mathrm{N}} / \mathrm{mM}$ & $m_{\mathrm{Si}} / \mu \mathrm{g}$ & $\sigma / \mu \mathrm{g}$ & $n$ \\
\hline C3N6 & 1 & 9,0 & 2,6 & 32 \\
C3N6 & 2 & 31,0 & 7,4 & 4 \\
C3N12 & 1 & 14,0 & 1,5 & 20 \\
C3N12 & 2 & 32,7 & 3,8 & 8 \\
C3N18 & 1 & 22,0 & 1,7 & 32 \\
C3N18 & 2 & 57,2 & 8,0 & 4
\end{tabular}

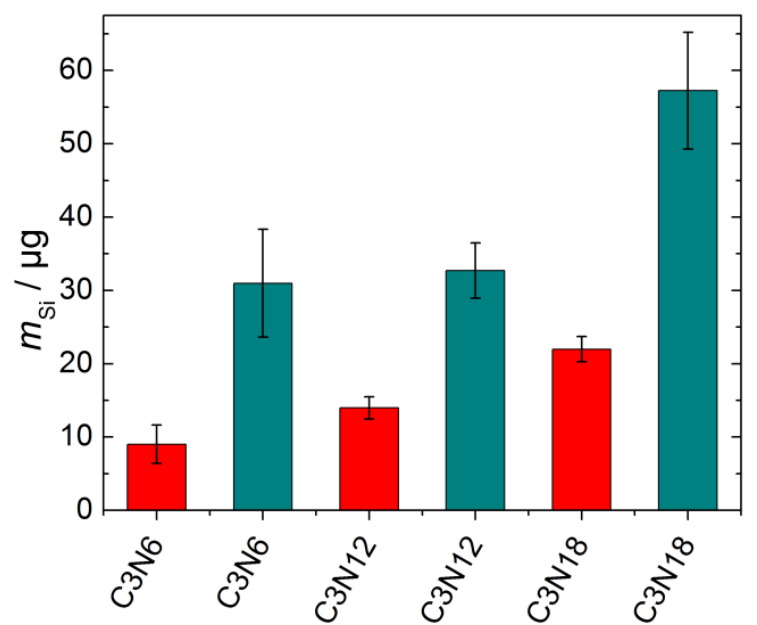

Abbildung 4.14 Zusammenfassung der mittels $\beta$-Molybdatmethode ermittelten Massen $m_{\mathrm{Si}}$ mit $c_{\mathrm{N}}$ : $1 \mathrm{mM}$ und 2 mM. Reaktionsbedingungen: $30 \mathrm{mM}$ Phosphatpuffer $\mathrm{pH}$ 6,8, $c_{(\mathrm{SiOH})_{4}}=100 \mathrm{mM}$, Reaktionszeit: $10 \mathrm{~min}$.

\subsubsection{Alkylgruppe der Polyamine}

Nachdem sich die Experimente aus Kapitel 4.2.1 mit dem Einfluss der Polyaminkettenlänge auf biomimetische Silicapräzipitationen beschäftigt haben, wird nachfolgend vorgestellt, wie sich Variationen der Alkylgruppe zwischen den Aminoeinheiten auswirken. $\mathrm{Zu}$ diesem Zweck wurden die unter 4.1 vorgestellten Substanzen C2N7, C3N7 sowie C4N7 miteinander verglichen. Die experimentell ermittelten Siliciummassen sind in Abbildung 4.15 dargestellt. Das geringste Präzipitationspotential hat bei den vorliegenden Bedingungen das Polyamin C2N7, dessen Aminoeinheiten über Ethylgruppen verbunden sind. Für dieses wurden 1,4 $\mu \mathrm{g}$ Si detektiert. Der Austausch der Ethyl- durch Propylgruppen steigert diese Menge um das fast achtfache auf 10,6 $\mu \mathrm{g}$. Als am effektivsten erwies sich Verbindung C4N7, die Butylgruppen besitzt. Mit ihr wurde unter identischen Bedingungen Silica präzipitiert, welches 21,6 $\mu \mathrm{g} \mathrm{Si}$ enthielt. 


\begin{tabular}{lccc}
\hline & $m_{\mathrm{Si}} / \mu \mathrm{g}$ & $\sigma / \mu \mathrm{g}$ & $n$ \\
\hline C2N7 & 1,4 & 0,6 & 7 \\
C3N7 & 10,6 & 3,7 & 8 \\
C4N7 & 21,6 & 2,2 & 8
\end{tabular}

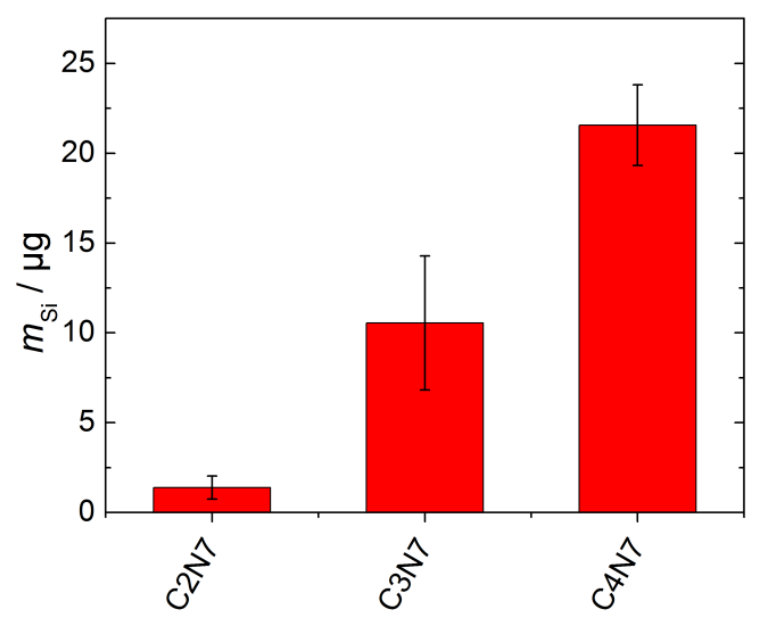

Abbildung 4.15 Mit den Polyaminen C2N7, C3N7 und C4N7 präzipitierte Siliciummassen $m_{\mathrm{Si}}$ mit $\sigma$ Standardabweichung und $n$ Anzahl der Messungen. Reaktionsbedingungen: $30 \mathrm{~mm}$ Phosphatpuffer pH 6,8, $c_{\mathrm{N}}=1 \mathrm{mM}, c_{(\mathrm{SiOH})_{4}}=100 \mathrm{mM}$, Reaktionszeit: $10 \mathrm{~min}$.

\section{Silicapräzipitationen mit C2N7}

Bei der Präzipitationsreaktion mit dem Polyamin C2N7 entsteht nur sehr wenig Silica, was aus Abbildung 4.15 ersichtlich wird. Dementsprechend wurde bei der rasterelektronenmikroskopischen Untersuchung kaum Material detektiert (Abbildung 4.16 A-D).
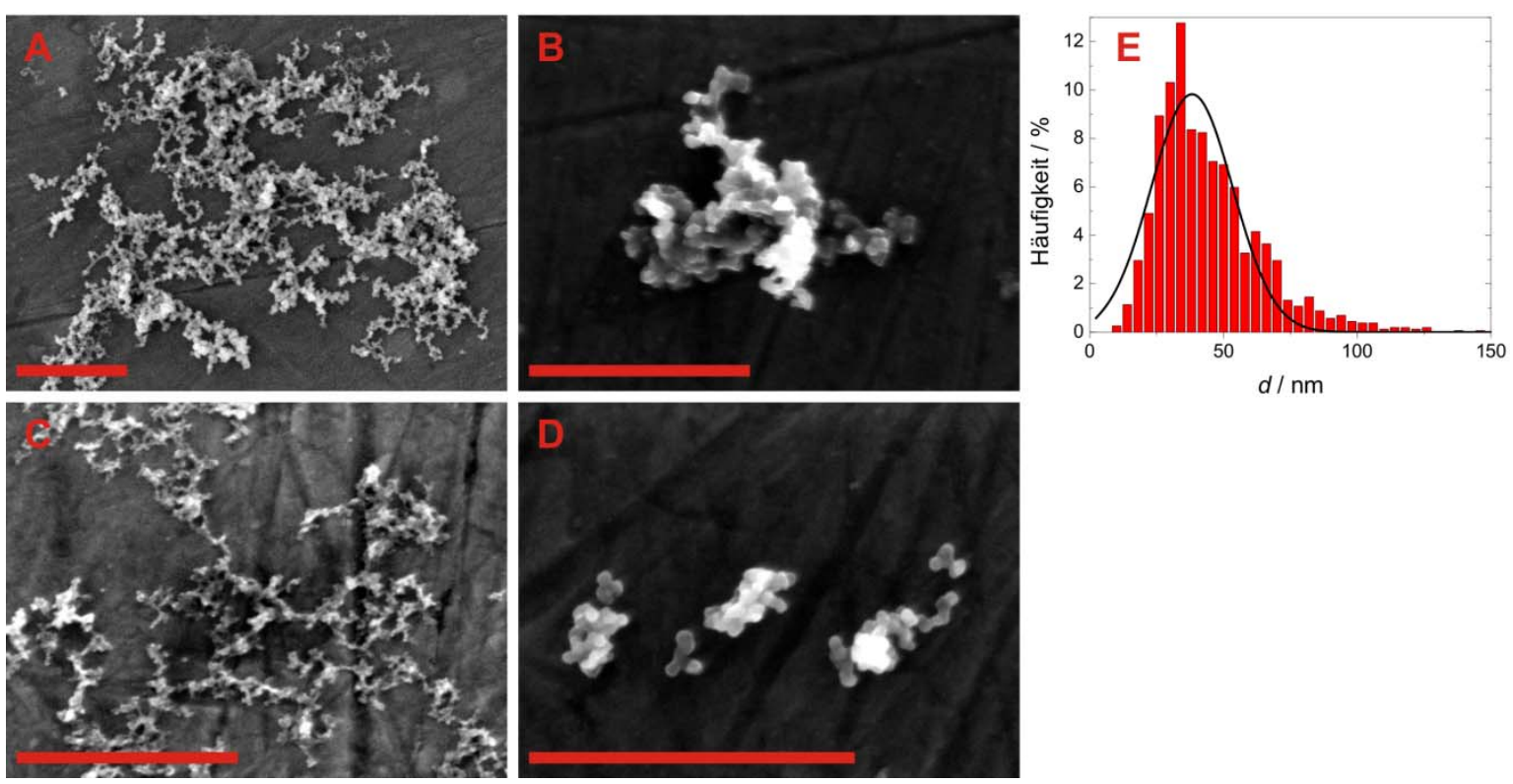

Abbildung 4.16 A-D: Repräsentative SEM-Aufnahmen von Silicapräzipitaten, deren Bildung durch Zugabe von $\mathrm{Si}(\mathrm{OH})_{4} \mathrm{zu}$ Lösungen von $\mathrm{C} 2 \mathrm{~N} 7$ induziert wurde. Maßstabsbalken: $1 \mu \mathrm{m}$. E: Im Histogramm sind die Kugeldurchmesser $d$ [1590 Kugeln, Binbreite: $4 \mathrm{~nm}$ ] aufgetragen. An die Daten wurde eine Gaußverteilung angepasst. Der hierbei ermittelte Mittelwert beträgt $d=(38 \pm 15) \mathrm{nm}$. Reaktionsbedingungen: $30 \mathrm{~mm}$ Phosphatpuffer $\mathrm{pH} 6,8, c_{\mathrm{N}}=1 \mathrm{mM}, c_{(\mathrm{SiOH})_{4}}=100 \mathrm{mM}$, Reaktionszeit: $10 \mathrm{~min}$. 
Die wenigen Partikel, die gefunden wurden, sind von sehr kleinem Durchmesser, was sich auch im abgebildeten Histogramm (Abbildung 4.16 E) widerspiegelt. Durch Anpassung einer Normalverteilung an die Daten ergab sich ein durchschnittlicher Kugeldurchmesser von $d=(38 \pm 15) \mathrm{nm}$. Die Partikel liegen zwar in Netzwerken vor, aber sind noch weitestgehend voneinander separiert (A/D). An einigen Stellen treten hingegen Anhäufungen auf, in denen die einzelnen Kugeln nicht mehr deutlich unterschieden werden können (B).

\section{Silicapräzipitationen mit C3N7}

Unter Verwendung von C3N7 entstehen Präzipitate in Form von Kugeln, deren Oberfläche ebenmäßig ist, was aus den SEM-Bildern in Abbildung 4.17 ersichtlich wird. Ihre Verteilung erscheint homogen, es zeigen sich jedoch neben der Hauptpopulation an Kugeln, die einen mittleren Durchmesser von $d_{2}=(122 \pm 34) \mathrm{nm}$ aufweisen, auch kleinere Partikel mit $d_{1}=(36 \pm 8) \mathrm{nm}$. Diese sind sowohl in Aufnahmen B und C erkennbar als auch im Histogramm (Abbildung 4.17 E).
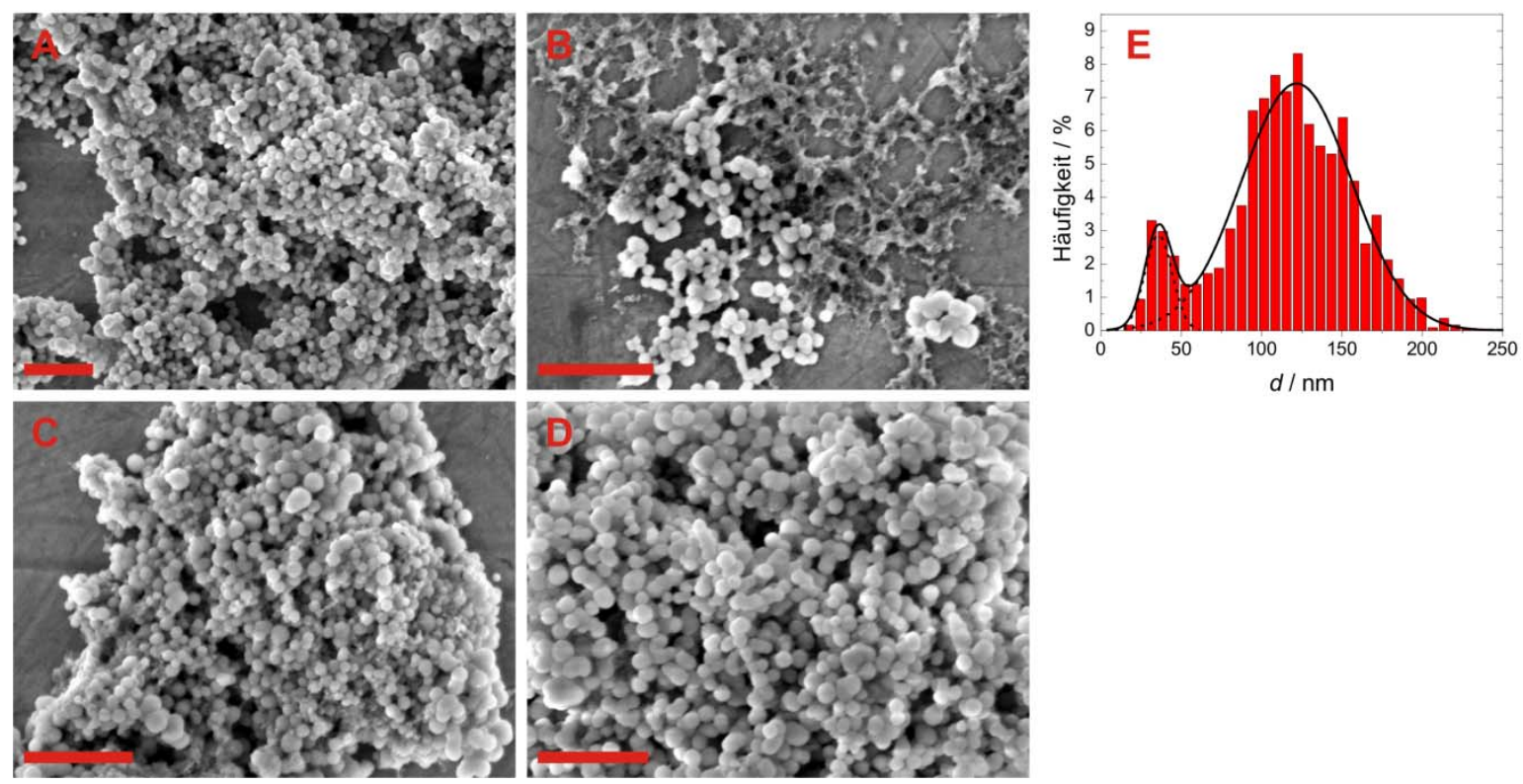

Abbildung 4.17 A-D: Repräsentative SEM-Aufnahmen von Silicapräzipitaten, entstanden durch Zugabe von $\mathrm{Si}(\mathrm{OH})_{4} \mathrm{zu}$ einer Lösung von C3N7. Maßstabsbalken: $1 \mu \mathrm{m}$. E: Histogramm der auftretenden Kugeldurchmesser $d$ [2452 Kugeln, Binbreite: $7 \mathrm{~nm}$ ]. Die gestrichelten Linien geben die Ergebnisse der Anpassung zweier Gaußverteilungen an die Daten wieder, ihre Summe ist in der durchgehenden Linie wiedergegeben. Die Mittelwerte betragen $d_{1}=(36 \pm 8) \mathrm{nm}$ und $d_{2}=(122 \pm 34) \mathrm{nm}$. Reaktionsbedingungen: $30 \mathrm{~mm}$ Phosphatpuffer $\mathrm{pH} 6,8, c_{\mathrm{N}}=1 \mathrm{mM}, c_{(\mathrm{SiOH})_{4}}=100 \mathrm{mM}$, Reaktionszeit: $10 \mathrm{~min}$. 


\section{Silicapräzipitationen mit C4N7}

Mit dem Polyamin C4N7 wurde mehr Silica präzipitiert als mit C2N7 oder C3N7. Trotzdem lassen sich in den rasterelektronenmikroskopischen Aufnahmen aus Abbildung 4.18 A-D ähnliche Strukturen erkennen. Es treten wiederum gleichmäßig aussehende Kugeln auf, deren Durchmesser sich um einen Mittelwert verteilen. Die statistische Untersuchung (Abbildung 4.18 E) ergab einen Wert für den mittleren Durchmesser der Kugeln von $d=(172 \pm 35) \mathrm{nm}$.
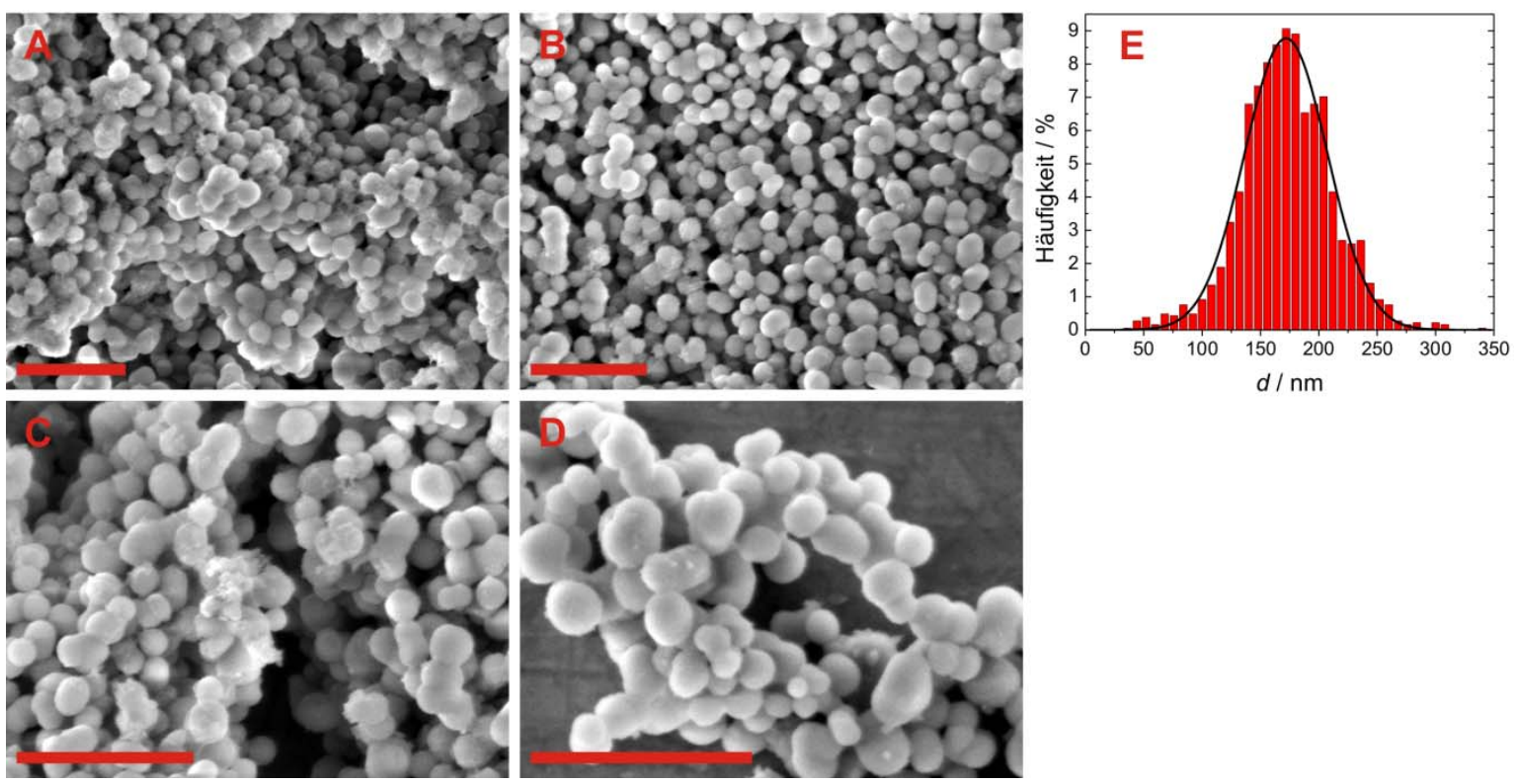

Abbildung 4.18 A-D: Auswahl an SEM-Aufnahmen, in denen Silica zu sehen ist, das durch die Zugabe von $\mathrm{Si}(\mathrm{OH})_{4}$ zu einer Lösung von C4N7 präzipitiert wurde. Maßstabsbalken: $1 \mu \mathrm{m}$. E: Die Durchmesser $d$ der Kugeln wurden ermittelt und in einem Histogramm [1853 Kugeln, Binbreite: $8 \mathrm{~nm}$ ] aufgetragen. Die schwarze Linie gibt das Resultat der Anpassung einer Gaußverteilung an die Daten wieder. Der Mittelwert beträgt $d=(172 \pm 35) \mathrm{nm}$. Reaktionsbedingungen: $30 \mathrm{mM} \quad$ Phosphatpuffer $\quad \mathrm{pH} 6,8, \quad c_{\mathrm{N}}=1 \mathrm{mM}$, $c_{(\mathrm{SiOH})_{4}}=100 \mathrm{mM}$, Reaktionszeit: $10 \mathrm{~min}$.

Die hier untersuchten Polyamine, die jeweils sieben Aminoeinheiten aufweisen und sich in ihrer hydrophoben Alkylgruppe unterscheiden, besitzen unterschiedliche präzipitationsinduzierende Eigenschaften. Mit allen drei Substanzen wurden kugelförmige Partikel gebildet, deren mittlere Durchmesser von C2N7 über C3N7 bis C4N7 ansteigen. Entsprechend nimmt die Masse an präzipitiertem Silicium in der gleichen Reihenfolge deutlich $\mathrm{zu}$.

\section{Zusammenfassung}

Beim Vergleich von Polyaminen mit unterschiedlichen Alkylgruppen zwischen den Aminoeinheiten (C2N7, C3N7, C4N7) bezüglich ihrer $\mathrm{SiO}_{2}$-präzipitierenden Funktion 
zeichneten sich mehrere Unterschiede ab. Zum einen steht die Menge an Silica, welches präzipitiert wird, in Zusammenhang mit den Strukturen der Substanzen, zum anderen gibt es auch Auswirkungen auf die Partikelgröße. Wie auch bei den Polyaminen mit unterschiedlicher Gesamtkettenlänge, wurden die drei Verteilungen statistisch miteinander verglichen. Die Ergebnisse der Mann-Whitney-U-Tests besagen, dass sich die drei Verteilungen mit hoher Signifikanz $(P<0,001)$ voneinander unterscheiden. Dabei wurden ausschließlich Kugeln gefunden, filamentartiges $\mathrm{SiO}_{2}$ wie bei C3N18 trat nicht auf.

\subsubsection{N-Methylierung der Polyamine}

Bekanntermaßen beeinflusst die Methylierung von Aminogruppen in Polyaminen die Kinetik von Kieselsäurepräzipitationsreaktionen, die von diesen Polyaminen induziert werden. ${ }^{102}$ Um nähere Informationen darüber zu erhalten, wie sich Methylierung auf die Menge an $\mathrm{SiO}_{2}$ sowie dessen mikroskopischer Struktur auswirkt, wurde C3N12Me untersucht und mit seinem unmethylierten Analogon C3N12 verglichen. Die präzipitierte Si-Masse wurde mittels der $\beta$-Molybdatmethode ermittelt. Die $N$-Methylierung bewirkt ungefähr eine Verdopplung der präzipitierten Si-Menge von 14,0 $\mu \mathrm{g}$ für C3N12 auf 31,0 $\mu \mathrm{g}$ im Fall von C3N12Me (Abbildung 4.19).

\begin{tabular}{cccc}
\hline & $m_{\mathrm{si}} / \mu \mathrm{g}$ & $\sigma / \mu \mathrm{g}$ & $n$ \\
\hline C3N12 & 14,0 & 1,5 & 20 \\
C3N12Me & 31,0 & 1,5 & 2
\end{tabular}

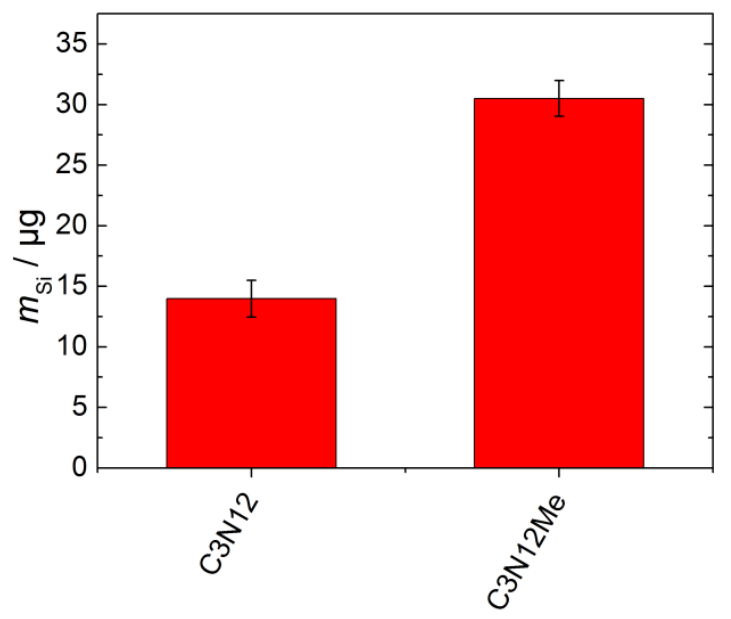

Abbildung 4.19 Bei Präzipitationsreaktionen mit den Polyaminen C3N12 und C3N12Me wurde mittels $\beta$-Molybdatmethode die Masse an Silicium $m_{\mathrm{Si}}(\sigma$ : Standardabweichung, $n$ : Anzahl der Messungen) ermittelt. Reaktionsbedingungen: $30 \mathrm{mM}$ Phosphatpuffer $\mathrm{pH} 6,8, c_{\mathrm{N}}=1 \mathrm{mM}, c_{(\mathrm{SiOH})_{4}}=100 \mathrm{mM}$, Reaktionszeit: $10 \mathrm{~min}$.

Rasterelektronenmikroskopische Messungen eines $\mathrm{SiO}_{2}$-Präzipitats, dessen Bildung durch C3N12Me induziert wurde, zeigen gleichmäßig geformte kugelartige Strukturen (Abbildung 4.20 A-E). Diese liegen in zwei Größen vor, deren Mittelwerte zu 
$d_{l}=(59 \pm 24) \mathrm{nm}$ sowie $d_{2}=(183 \pm 27) \mathrm{nm}$ bestimmt wurden. Die kleinen Kugeln haben dabei einen Anteil von ca. $30 \%$ an der Gesamtzahl. Diese Ergebnisse sind in einem Histogramm in Abbildung 4.20 F dargestellt. Im Vergleich dazu lagen die Durchmesser der mit C3N12 gebildeten Partikel im Bereich einer einzigen Normalverteilung um $(169 \pm 23) \mathrm{nm}$.
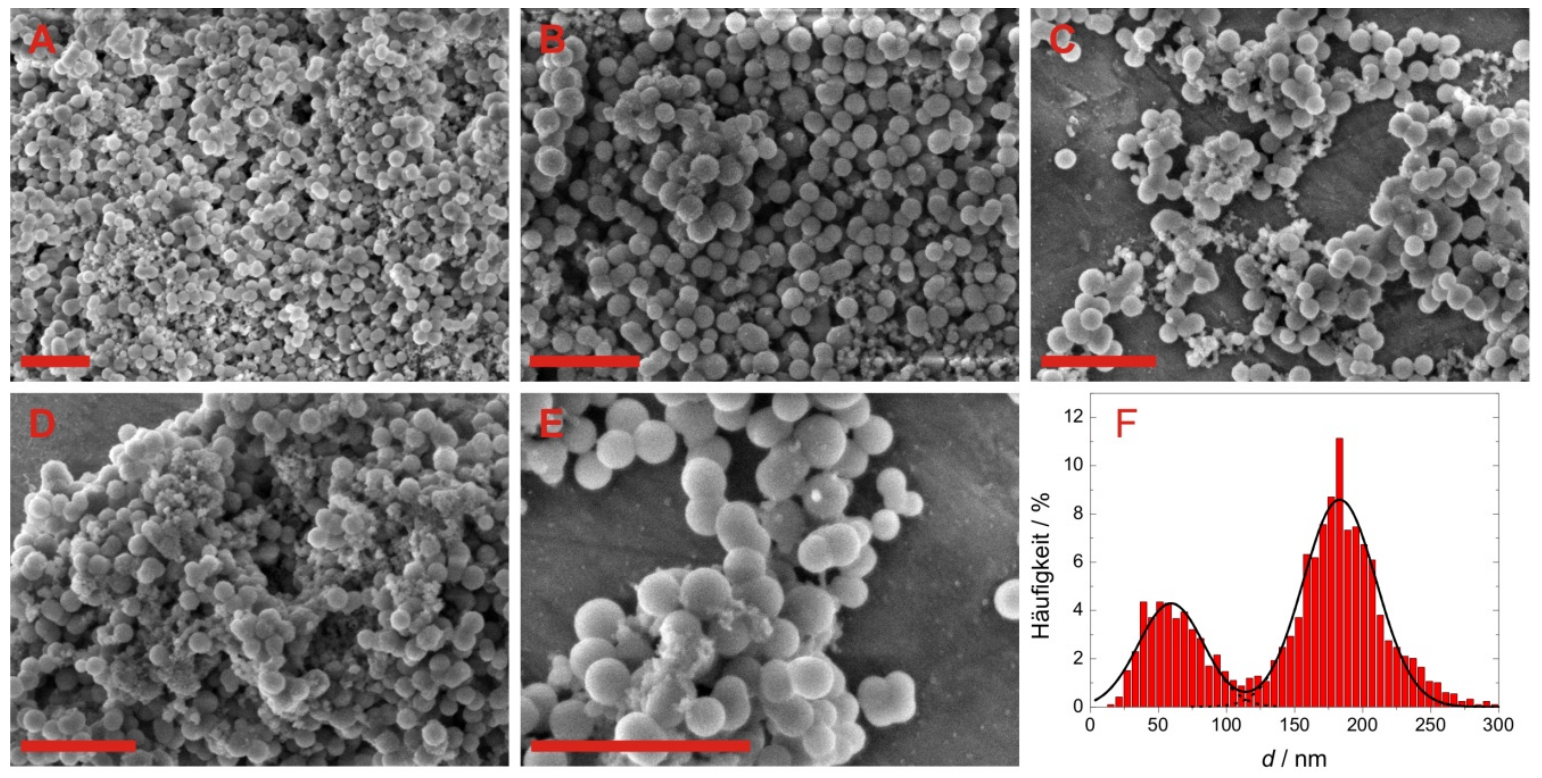

Abbildung 4.20 A-E: SEM-Aufnahmen eines Silicapräzipitats, das durch Zugabe von Monokieselsäure zu einer Lösung von C3N12Me gebildet wurde. Maßstabsbalken: $1 \mu \mathrm{m}$. F: Histogramm der auftretenden Kugeldurchmesser $d$ [3125 Kugeln, Binbreite: $6 \mathrm{~nm}$. Gestrichelte Linien: Anpassung zweier Gaußverteilungen an die Daten; durchgehende Linie: Summe aus den beiden Einzelverteilungen. Die Mittelwerte betragen $d_{l}=(59 \pm 24) \mathrm{nm}$ sowie $d_{2}=(183 \pm 27) \mathrm{nm}$. Reaktionsbedingungen: $30 \mathrm{mM}$ Phosphatpuffer $\mathrm{pH} 6,8, c_{\mathrm{N}}=1 \mathrm{mM}, c_{(\mathrm{SiOH})_{4}}=100 \mathrm{mM}$, Reaktionszeit: $10 \mathrm{~min}$.

\subsection{Einfluss von Phosphationen auf}

\section{Präzipitationsreaktionen}

In verschiedenen Studien wurde auf die Bedeutung von multivalenten Anionen für die Aggregation von Silaffinen oder Polyaminen und anschließende $\mathrm{SiO}_{2}$-Präzipitationen hingewiesen, ohne die in den meisten Fällen keine Reaktionen stattfinden. ${ }^{31,65,105}$ Es gibt jedoch auch Arbeiten, in denen Silicabildung mit Polyaminen unter Abwesenheit von Anionen wie Phosphat $\left(\mathrm{P}_{\mathrm{i}}\right)$ oder Sulfat beobachtet wurde. ${ }^{102,106}$ Bisher wurden diverse $\mathrm{P}_{\mathrm{i}}: \mathrm{N}-$ Verhältnisse eingesetzt, welche von ungefähr von $0,3: 1$ bis zu 5:1 reichten. Brunner et al. identifizierten dabei ein $\mathrm{P}_{\mathrm{i}}$ :N-Verhältnis von ca. $0,5: 1{ }^{41}$ Die veröffentlichten Ergebnisse sind relativ inhomogen und es wurden immer nur einzelne Polyamine 
beziehungsweise Silaffine untersucht. Im Rahmen dieser Arbeit stehen mehrere Polyamine, darunter C3N6, C3N12 und C3N18, zur Verfügung, die sich, wie im vorhergehenden Kapitel aufgezeigt wurde, in ihren silicapräzipitierenden Eigenschaften unterscheiden. Eine systematische Variation der Phosphatkonzentration und anschließende Untersuchung von gebildeter Siliciummasse sowie Morphologie des Silica könnte weitere Erkenntnisse $\mathrm{zu}$ den bei der Biomineralisation ablaufenden Prozessen liefern. Alle verwendeten Polyaminlösungen enthielten $1 \mathrm{mM}$ Stickstoff. Der Phosphatpuffer wurde durch einen Acetatpuffer ( 30 mM, pH 6,8) ersetzt und die entsprechenden Konzentrationen an Phosphat (0,25 mM, 0,5 mM, 0,75 mM, $3 \mathrm{~mm}, 10 \mathrm{mM}$ und $30 \mathrm{~mm})$ wurden durch Zugabe aus einem $500 \mathrm{~mm}$ Phosphatpuffer pH 6,8 angepasst. Unter diesen Bedingungen wurden mit den Polyaminen Präzipitationsreaktionen durchgeführt und die jeweils gefällte Menge an Silicium mit dem $\beta$-Molybdattest bestimmt. Diese Tests wurden für jede Substanz und jede Phosphatkonzentration viermal wiederholt. Die Resultate sind in Abbildung 4.21 dargestellt. Es ist zu beachten, dass die x-Achse zur besseren Übersicht für kleine Phosphatkonzentrationen linear, bei größeren logarithmisch aufgetragen ist.

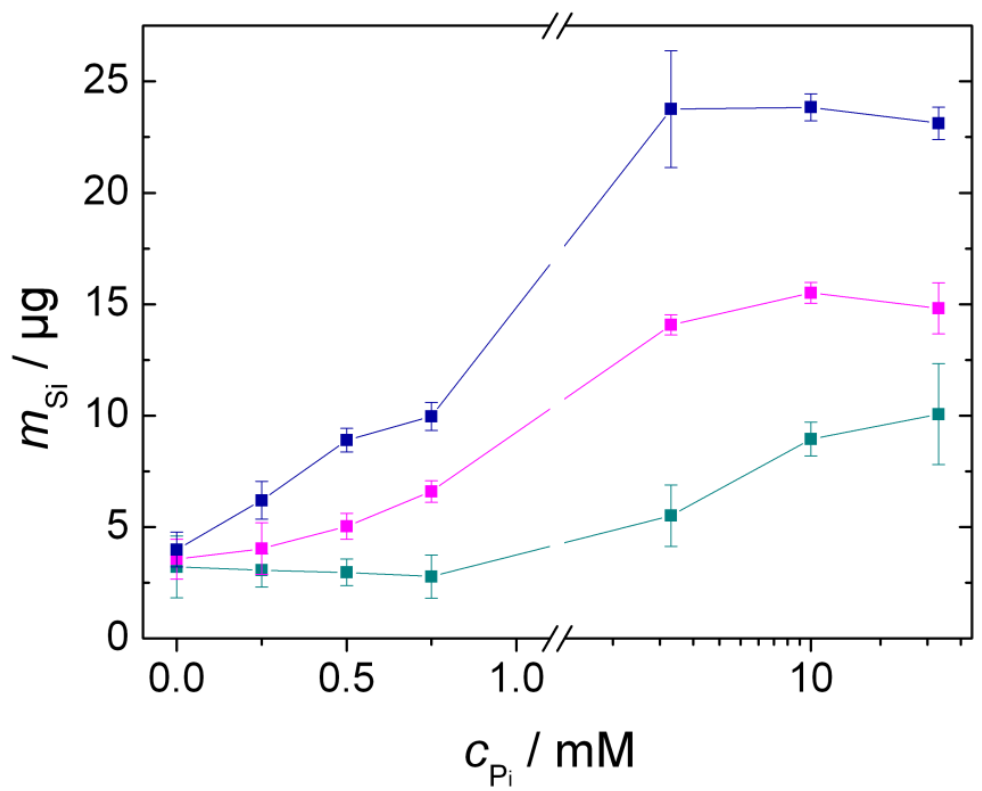

Abbildung 4.21 Überblick über die mittels $\beta$-Molybdatmethode bestimmten Massen $m_{\mathrm{Si}}$ an präzipitiertem Silicium bei verschiedenen Phosphatkonzentrationen $c_{\mathrm{Pi}}$ für die Polyamine - C3N6 - C3N12 - C3N18. Reaktionsbedingungen: $30 \mathrm{mM}$ Acetatpuffer $\mathrm{pH}$ 6,8, $c_{\mathrm{N}}=1 \mathrm{mM}, c_{(\mathrm{SiOH})_{4}}=100 \mathrm{mM}$, Reaktionszeit: $10 \mathrm{~min}$.

Bei allen drei Polyaminen tritt eine Zunahme an präzipitiertem Si mit Erhöhung der Phosphatkonzentration auf. Der Anfangswert für $0 \mathrm{~mm}$ Phosphat liegt für alle bei $(3,2-4,0) \mu \mathrm{gSi}$. Es ist wahrscheinlich, dass dieser Wert durch geringe Mengen an Kieselsäure, die durch den Waschvorgang nicht vollständig entfernt werden konnten, sowie einen kleinen Anteil 
an polymerisiertem $\mathrm{SiO}_{2}$ zustande kommt. Aufgrund des kleinen Wertes kann hier nicht von spezifischen Präzipitationsreaktionen ausgegangen werden.

Im Fall von C3N6 ist ab $c_{\mathrm{Pi}}=3 \mathrm{~mm}$ ein Anstieg erkennbar, während sich zwischen $10 \mathrm{mM}$ und $30 \mathrm{~mm}$ keine wesentliche Änderung mehr zeigt. Auch bei C3N12 und C3N18 wurden ähnliche Verläufe gefunden. Zur besseren Vergleichbarkeit wurden an die Daten Geraden angepasst, deren Parameter zusätzliche Informationen liefern (Abbildung 4.22).
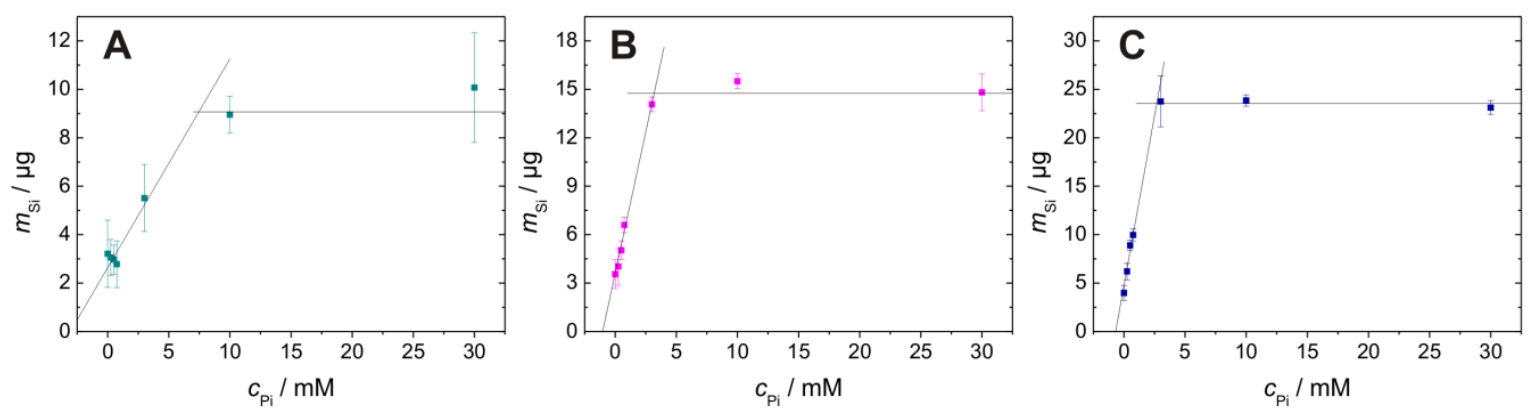

Abbildung 4.22 Lineare Anpassungen an die Daten der phosphatabhängigen Bestimmungen der präzipitierten Siliciummassen für die Polyamine A C3N6, B C3N12 und C C3N18.

Für C3N6 beträgt die Steigung im Bereich kleiner Phosphatkonzentrationen $(0,9 \pm 0,5) \mu \mathrm{g} \mathrm{Si} / \mathrm{mM} \mathrm{P}_{\mathrm{i}}$. Ab dem Schnittpunkt der beiden Geraden, der bei 7,5 mM $\mathrm{P}_{\mathrm{i}}$ ( $\mathrm{P}_{\mathrm{i}}: \mathrm{N}-$ Verhältnis: 7,5:1) liegt, ändert sich $m_{\mathrm{Si}}$ kaum und wurde $\mathrm{zu}(9,1 \pm 0,7) \mu \mathrm{g}$ bestimmt. Die Steigung im phosphatabhängigen Bereich ist bei $\mathrm{C} 3 \mathrm{~N} 12$ höher und macht $(3,5 \pm 0,2) \mu \mathrm{g} \mathrm{Si} / \mathrm{mm} \mathrm{P}_{\mathrm{i}}$ aus. Ab 3,2 mM $\mathrm{P}_{\mathrm{i}}\left(\mathrm{P}_{\mathrm{i}}: \mathrm{N}\right.$-Verhältnis: 3,2:1) ist eine Sättigung an Phosphationen erreicht und es ergibt sich eine maximale Siliciummassen von $(14,8 \pm 0,7) \mu \mathrm{g}$. Bei C3N18 werden mit 2,7 mM ( $\mathrm{P}_{\mathrm{i}}: \mathrm{N}-$ Verhältnis: 2,7:1) nochmals weniger Phosphationen benötigt, um die maximale Präzipitation zu erhalten. Ebenso ändert sich die Siliciummasse in Abhängigkeit von $c_{\mathrm{Pi}}$ mit $(7,0 \pm 0,8) \mu \mathrm{g} \mathrm{Si} / \mathrm{mM} \mathrm{P}_{\mathrm{i}}$ bei kleinen Phosphatmengen stärker. C3N18 präzipitiert, wie schon bekannt, die größte Silicamenge, die hier $(23,6 \pm 0,7) \mu \mathrm{g}$ Si entspricht. Zusammenfassend lässt sich feststellen, dass bei den Präzipitationen $m_{\mathrm{Si}}$ linear mit der Phosphatkonzentration steigt, wenn diese klein ist. Es gibt für jedes Polyamin eine bestimmte Phosphatkonzentration, ab der sich die Si-Masse nicht mehr ändert und eine Sättigung eintritt. Je mehr Aminoeinheiten in den Propylpolyaminen vorhanden sind, desto weniger multivalente Anionen scheinen nötig zu sein, für $\mathrm{SiO}_{2}$-Präzipitationen optimale Bedingungen zu schaffen. 


\section{Einfluss der Phosphatkonzentration auf Silicapräzipitationen mit C3N6}

Bei Phosphatkonzentrationen von $3 \mathrm{mM}, 10 \mathrm{~mm}$ und $30 \mathrm{~mm}$ wurde mit allen drei Propylpolyaminen präzipitiertes Silica rasterelektronenmikroskopisch analysiert, um die Phosphatabhängigkeit weiter zu charakterisieren. Wird C3N6 zur Präzipitationsinduktion eingesetzt, sind vor allem bei $3 \mathrm{mM} \mathrm{P}_{\mathrm{i}}$ Unterschiede $\mathrm{zu}$ den höheren Phosphatkonzentrationen erkennbar (Abbildung $4.23 \mathrm{~A} / \mathrm{B}$ ).
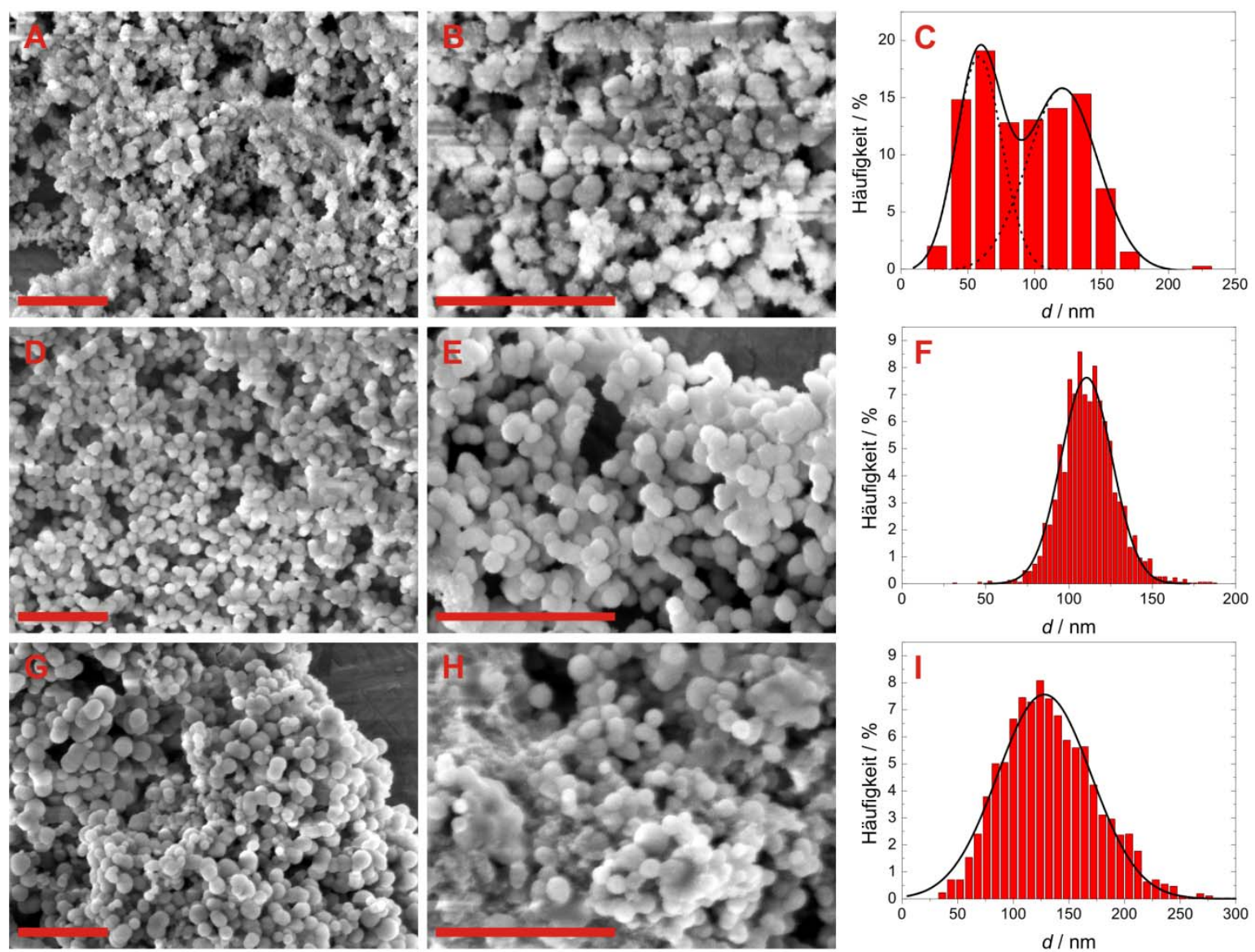

Abbildung 4.23: Überblick über SEM-Aufnahmen von Silica, dessen Bildung durch das Polyamin C3N6 in Anwesenheit von A/B $3 \mathrm{~mm}$ Phosphationen, D/E $10 \mathrm{~mm}$ Phosphationen, G/H $30 \mathrm{~mm}$ Phosphationen induziert wurde. Maßstabsbalken: $1 \mu \mathrm{m}$. C/F/I: Jeweilige zugehörige Histogramme der auftretenden Kugeldurchmesser $d$. Die Linien geben die Resultate der Anpassung einer oder mehrerer Gaußverteilungen an die Daten wieder. C 398 Kugeln, Binbreite: $18 \mathrm{~nm}$. Mittelwerte $d_{l}=(58 \pm 17) \mathrm{nm}$ und $d_{2}=(121 \pm 26) \mathrm{nm}$. F 3028 Kugeln, Binbreite: $3 \mathrm{~nm}$. Mittelwert $d=(111 \pm 15) \mathrm{nm}$. I 2537 Kugeln, Binbreite: $8 \mathrm{~nm}$. Mittelwert $d=(128 \pm 42) \mathrm{nm}$. Reaktionsbedingungen: $30 \mathrm{mM}$ Acetatpuffer $\mathrm{pH} \mathrm{6,8,} c_{\mathrm{N}}=1 \mathrm{mM}, c_{(\mathrm{SiOH})_{4}}=100 \mathrm{mM}$, Reaktionszeit: $10 \mathrm{~min}$.

Insgesamt findet sich in diesen SEM-Proben wenig Silica. Dieses liegt teilweise in kugelartigen Strukturen zweier Größenverteilungen nebeneinander vor $\left(d_{1}=(58 \pm 17) \mathrm{nm}\right.$, $\left.d_{2}=(121 \pm 26) \mathrm{nm}\right)$, wie das Histogramm (Abbildung $\left.4.23 \mathrm{C}\right)$ wiedergibt. Zusätzlich ist viel Material erkennbar, das keine definierte Struktur aufweist und oftmals die Kugeln bedeckt, was zu einem inhomogenen Gesamtbild führt. Schon bei $10 \mathrm{mM} \mathrm{P}_{\mathrm{i}}$ werden die 
Strukturen definierter, es finden sich ausschließlich Silicakugeln, die eine glatte Schale besitzen (D/E). Ihre Durchmesser entsprechen einer Normalverteilung mit einem Mittelwert von $(111 \pm 15) \mathrm{nm}$ (Histogramm Abbildung $4.23 \mathrm{~F}$ ). Ebenso verhält es sich bei einer Phosphatkonzentration von $30 \mathrm{mM}(\mathrm{G} / \mathrm{H})$, wobei hier der Kugeldurchmesser mit $(128 \pm 42) \mathrm{nm}$ größer und über einen breiteren Bereich verteilt ist (Abbildung 4.23 I). Die Erhöhung der Phosphatkonzentration bewirkt in diesem Fall eine Vergrößerung der Kugeln. Unterhalb von $10 \mathrm{mM} \mathrm{P}_{\mathrm{i}}$ werden in geringerem Maße gleichmäßige Strukturen gebildet. Somit beeinflusst die Phosphatkonzentration mit C3N6 sowohl die gebildeten Massen an Silica sowie dessen Morphologie.

\section{Einfluss der Phosphatkonzentration auf Silicapräzipitationen mit C3N12}

Ein Vergleich von Silicapräzipitaten, deren Bildung mit C3N12 in Anwesenheit unterschiedlicher Phosphatkonzentrationen induziert wurde, zeigt weniger Unterschiede, als dies mit C3N6 der Fall ist. Sowohl mit $3 \mathrm{~mm}, 10 \mathrm{~mm}$ als auch mit $30 \mathrm{~mm}$ Phosphat entsteht jeweils eine Population an gleichmäßig geformten Kugeln (Abbildung 4.24 A/B, $\mathrm{D} / \mathrm{E}, \mathrm{G} / \mathrm{H})$. Während sich für $3 \mathrm{mM} \mathrm{P}_{\mathrm{i}}$ eine mittlere Kugelgröße von $(93 \pm 22) \mathrm{nm}$ ergibt (Histogramm Abbildung 4.24 C), liegt dieser mit $10 \mathrm{mM} \mathrm{P}_{\mathrm{i}}$ bei $(101 \pm 21) \mathrm{nm}$ (Abbildung 4.24 F). Fast identisch zu $10 \mathrm{~mm} \mathrm{P}_{\mathrm{i}}$ ist die Verteilung für entsprechend $30 \mathrm{~mm} \mathrm{P}_{\mathrm{i}}$. Die Kugeln haben einen durchschnittlichen Durchmesser von (106 \pm 24$)$ nm (Abbildung 4.24 I). Wie eingangs erwähnt, wirkt sich bei C3N12 eine Variation der Phosphatkonzentration im Bereich von 3-30 mM nicht stark auf die Morphologie des Silica aus, so wie auch die gemessenen Si-Massen nicht stark voneinander abweichen (Abbildung 4.21). 

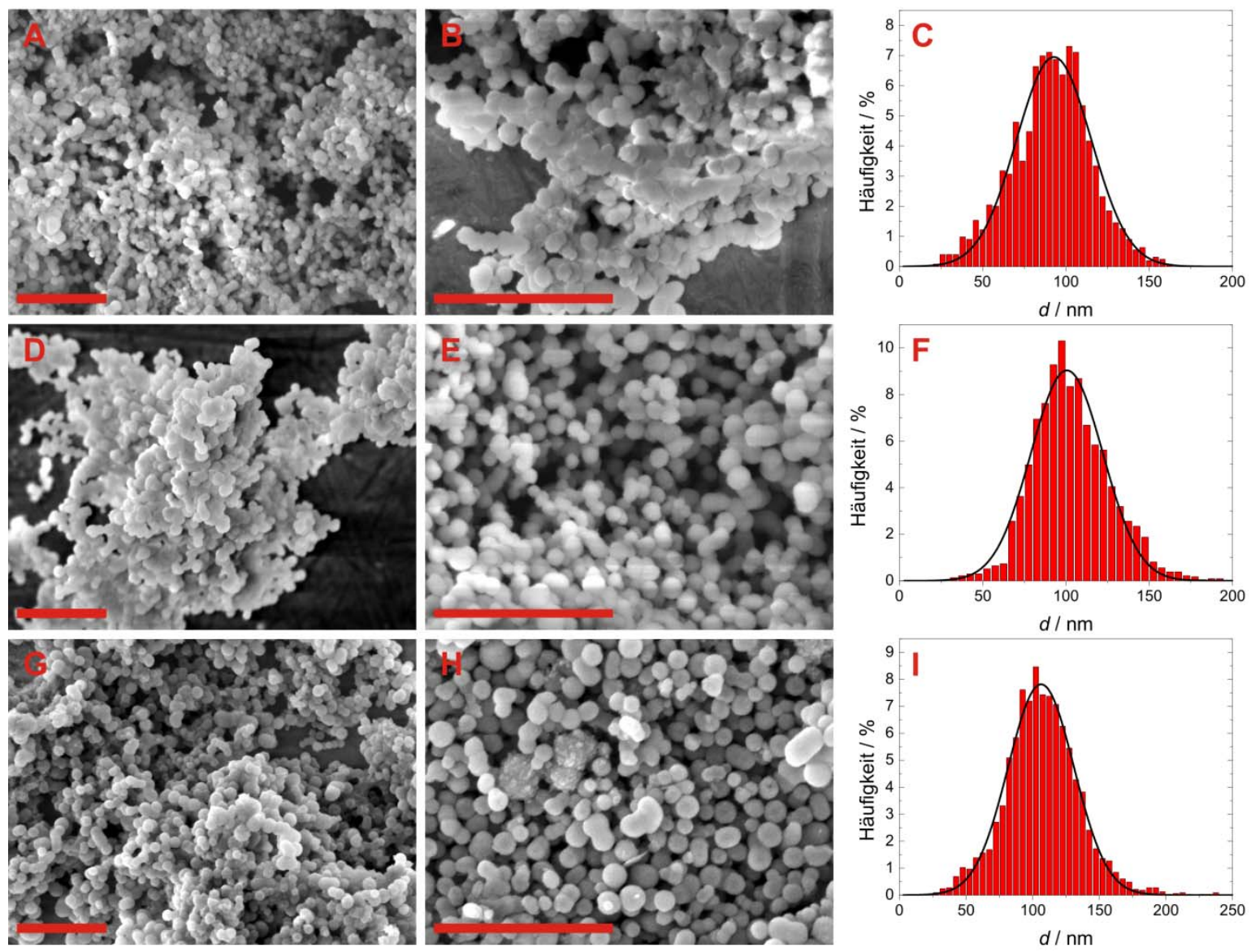

Abbildung 4.24 Repräsentative SEM-Aufnahmen von Silica, das nach Präzipitation mit dem Polyamin C3N12 in Anwesenheit von A/B $3 \mathrm{mM}, \mathbf{D} / \mathbf{E} 10 \mathrm{mM}$, oder G/H $30 \mathrm{mM}$ Phosphationen entstand. Maßstabsbalken: $1 \mu \mathrm{m}$. C/F/I: Die auftretenden Kugeldurchmesser $d$ sind in den jeweiligen Histogrammen dargestellt. An die Daten wurden Gaußverteilung angepasst (schwarze Linien). C 2546 Kugeln, Binbreite: $4 \mathrm{~nm}$. Mittelwert $d=(93 \pm 22) \mathrm{nm}$. F 2349 Kugeln, Binbreite: $5 \mathrm{~nm}$. Mittelwert $d=(101 \pm 21) \mathrm{nm}$. I 3321 Kugeln, Binbreite: $5 \mathrm{~nm}$. Mittelwert $d=(106 \pm 24) \mathrm{nm}$. Reaktionsbedingungen: $30 \mathrm{~mm}$ Acetatpuffer $\mathrm{pH} 6,8, c_{\mathrm{N}}=1 \mathrm{mM}, c_{(\mathrm{SiOH})_{4}}=100 \mathrm{mM}$, Reaktionszeit: $10 \mathrm{~min}$.

\section{Einfluss der Phosphatkonzentration auf Silicapräzipitationen mit C3N18}

Im Gegensatz zu C3N12 hat die Veränderung der Phosphatkonzentration bei Verwendung von C3N18 zur Präzipitation drastische Auswirkungen auf die Morphologie des präzipitierten Silica, obwohl die Mengen davon nicht wesentlich beeinflusst werden (siehe Abbildung 4.21). Bei einer Phosphationenkonzentration von $3 \mathrm{~mm}$ entsteht ausschließlich kugelförmiges Silica. Die Partikel sind voneinander abgegrenzt, auch wenn sie teilweise aneinander gereiht sind (Abbildung 4.25 A/B). Ihre Größen liegen im Bereich einer engen Normalverteilung (Abbildung 4.25 C). Mit einem mittleren Durchmesser von $(80 \pm 18) \mathrm{nm}$ sind sie relativ klein. Bei Erhöhung von $c_{\mathrm{Pi}}$ auf $10 \mathrm{mM}$ treten neben Bereichen mit Kugeln (D) auch solche auf, in denen sich filamentartiges Silica befindet (E). Ebenso ist dort eine Population von kleineren $\mathrm{SiO}_{2}$-Partikeln vorhanden. Die Durchmesser der Kugeln wurden ausgewertet und es ergaben sich zwei voneinander getrennte Verteilungen(Abbildung 4.25F) 
um $d_{l}=(25 \pm 7) \mathrm{nm}$ beziehungsweise häufiger auftretend um $d_{2}=(130 \pm 19) \mathrm{nm}$. In Anwesenheit von $30 \mathrm{~mm}$ Phosphat ist die Tendenz zur Bildung von Filamenten noch stärker ausgeprägt $(\mathrm{G} / \mathrm{H})$. Wiederum treten neben den gleichmäßigen großen Kugeln mit $d_{2}=(154 \pm 46)$ kleinere auf, die einen Durchmesser von $d_{l}=(30 \pm 10) \mathrm{nm}$ aufweisen (Abbildung 4.25 I). Zusammenfassend lässt sich für C3N18 feststellen, dass eine Erhöhung von $c_{\mathrm{Pi}}$ von $3 \mathrm{mM}$ auf $30 \mathrm{mM}$ zwar nicht zu einer Erhöhung präzipitierten Si-Masse führt, wohl aber die Strukturen insofern verändert, dass die Größe von $\mathrm{SiO}_{2}$-Kugeln steigt und vermehrt Silicafilamente auftreten.
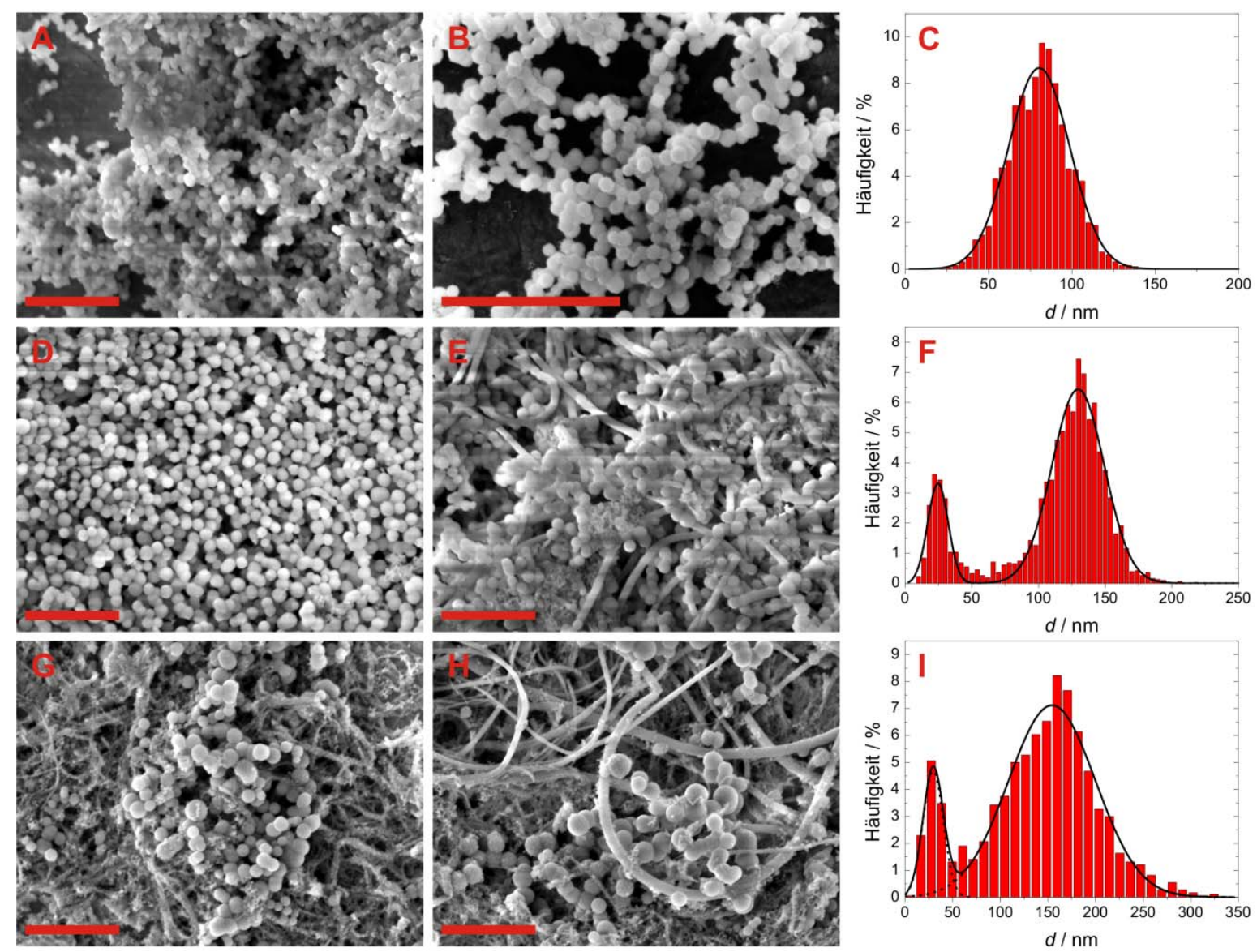

Abbildung 4.25 SEM-Aufnahmen von C3N18-Silicaproben, bei deren Präzipitation die Phosphatkonzentration variiert wurde: A/B $3 \mathrm{mM}$, D/E $10 \mathrm{mM}$ beziehungsweise G/H $30 \mathrm{~mm}$ Phosphationen. Maßstabsbalken: $1 \mu \mathrm{m}$. In $\mathbf{C} / \mathbf{F} / \mathbf{I}$ sind die zugehörigen Häufigkeiten der ermittelten Kugeldurchmesser $d$ sowie an diese Daten angepasste Gaußverteilungen dargestellt: C 1901 Kugeln, Binbreite: $4 \mathrm{~nm}$. Mittelwert $d=(80 \pm 18) \mathrm{nm}$. F 3091 Kugeln, Binbreite: $4 \mathrm{~nm}$. Mittelwerte $d_{l}=(25 \pm 7) \mathrm{nm}$ und $d_{2}=(130 \pm 19) \mathrm{nm}$. I 1598 Kugeln, Binbreite: $13 \mathrm{~nm}$. Mittelwerte $d_{1}=(30 \pm 10) \mathrm{nm}$ und $d_{2}=(154 \pm 46) \mathrm{nm}$. Reaktionsbedingungen: $30 \mathrm{mM}$ Acetatpuffer $\mathrm{pH} 6,8, c_{\mathrm{N}}=1 \mathrm{mM}, c_{(\mathrm{SiOH})_{4}}=100 \mathrm{mM}$, Reaktionszeit: $10 \mathrm{~min}$.

Die Veränderung der Konzentration von Phosphationen von (3 - 30) mM wirkte sich bei den Polyaminen C3N6, C3N12 und C3N18 nicht gleich aus. Mit C3N6 verursachte ihre Erhöhung eine Zunahme an gebildetem Silica, dessen Strukturen größer und vor allem homogener wurden. Weder quantitative noch strukturelle Effekte scheint die Variation der 
Anionenmenge im genannten Bereich mit C3N12 zu haben. Dies gilt auch im Fall von C3N18 bezüglich der Si-Masse, hier jedoch bewirkt die Erhöhung der Konzentration erhebliche Veränderungen der Strukturen von Kugeln hin zu Filamenten.

\subsection{Untersuchungen zur Reaktionsgeschwindigkeit}

Bei den bisher vorgestellten Experimenten wurde jeweils eine Reaktionszeit von zehn Minuten eingehalten. Da sich dabei unterschiedliche Mengen an Silica in Abhängigkeit vom verwendeten Polyamin abzeichneten (siehe Abbildungen 4.1, 4.14 und 4.19), stellte sich die Frage, ob auch die Kinetik der Reaktionen durch die Struktur der Polyamine beeinflusst wird. Zu diesem Zweck wurde mit den Verbindungen C3N6, C3N12 und C3N18 versucht, die Silicabildung zu unterschiedlichen Zeitpunkten abzubrechen und im Anschluss mit der $\beta$-Molybdatmethode quantitativ die Siliciummengen in den bis dahin entstandenen Präzipitaten zu ermitteln. Hierzu wurden die Reaktionsgefäße bei der jeweiligen Zeit zentrifugiert, die Überstände abgenommen und das Pellet mehrmals mit Wasser gewaschen, bevor es mit Natronlauge wieder in monomere Kieselsäure hydrolysiert wurde. Alle Experimente wurden mindestens viermal wiederholt. Die dabei bestimmten Ergebnisse sind in Abbildung 4.26 zusammengefasst.

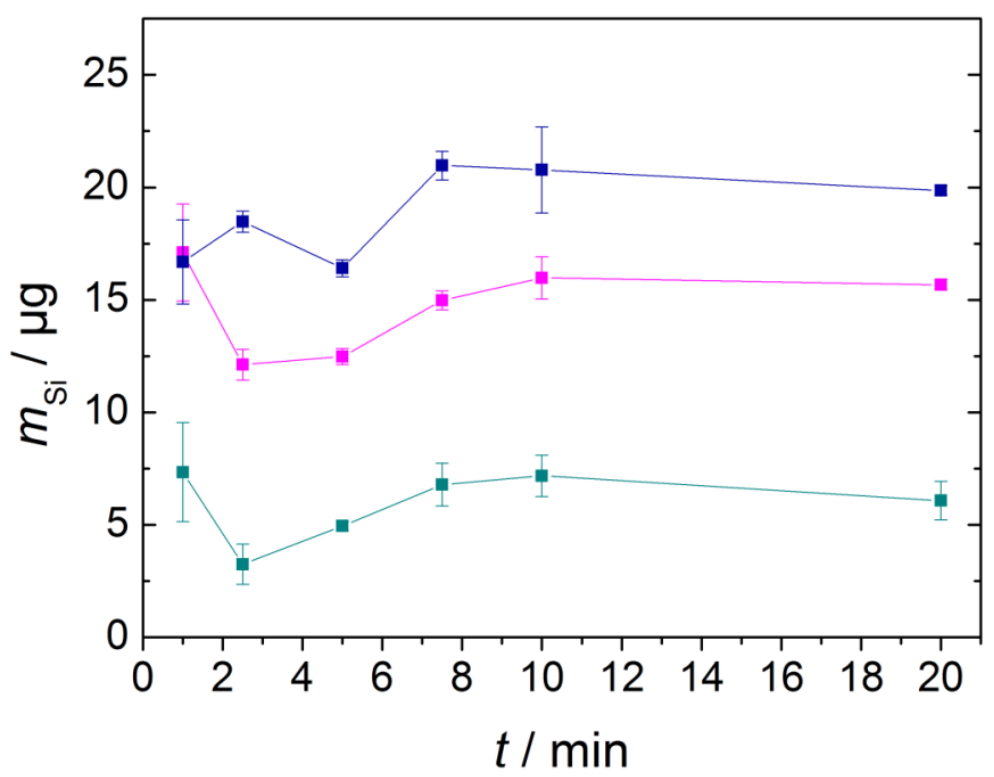

Abbildung 4.26 Überblick über die bei verschiedenen Reaktionszeiten $t$ mittels $\beta$-Molybdatmethode bestimmten Massen an präzipitiertem Silicium $m_{\mathrm{Si}}$ für die Polyamine $-\mathrm{C} 3 \mathrm{N6}-\mathrm{C} 3 \mathrm{~N} 12-\mathrm{C} 3 \mathrm{~N} 18$. Reaktionsbedingungen: $30 \mathrm{mM}$ Phosphatpuffer $\mathrm{pH} 6,8, c_{\mathrm{N}}=1 \mathrm{mM}, c_{(\mathrm{SiOH})_{4}}=100 \mathrm{mM}$. 
Bei allen drei Verbindungen wurde schon nach einer Minute Silicium detektiert. Für C3N6 liegt dieser Wert bei 7,4 $\mu \mathrm{g}$ Si. Nach 2,5 Minuten ist die Masse mit 3,3 $\mu \mathrm{g}$ niedriger und nimmt bis zu 7,2 $\mu \mathrm{g}$ nach zehn Minuten stetig zu. Nach zwanzig Minuten wiederum sinkt die Silicamenge leicht ab. Auch mit C3N12 ist der Wert für $t=1 \mathrm{~min}$ mit 17,1 $\mu \mathrm{g}$ der höchste in der Versuchsreihe detektierte. Wiederum zwischen 2,5 und zehn Minuten ist ein Anstieg von 12,1 $\mu \mathrm{g}$ auf 16,0 $\mu \mathrm{g}$ aufgetreten. Danach bleibt $m_{\mathrm{Si}}$ unverändert. Bei Einsatz von C3N18 liegt die Siliciummasse nach einer Minute bereits bei 16,7 $\mu$ g. Nach 2,5 Minuten nimmt sie leicht $\mathrm{zu}$, um bis zu fünf Minuten wieder abzunehmen. Bei 7,5 Minuten ist der Maximalwert von 21,0 $\mu$ g erreicht. Danach ändert sich die Menge nicht mehr wesentlich. Bei Betrachtung aller Daten zeigen sich keine spezifischen Zusammenhänge zwischen Silicamengen und Reaktionszeit. Eventuell wird die Reaktion durch die Zentrifugation der Proben nicht ausreichend gestoppt. Oder aber sie verläuft so schnell, dass schon nach einer Minute im wesentlichen die maximale Präzipitationsmenge erreicht ist und nachfolgend lediglich durch die Analysemethode bedingte Schwankungen auftreten. Wichtig für die vorliegende Arbeit ist, dass bei der für alle anderen Experimente verwendeten Zeit von zehn Minuten die Reaktionen abgeschlossen sind, wovon in Anbetracht der kinetischen Daten ausgegangen werden kann.

Mittels rasterelektronischen Messungen wurde zusätzlich die Morphologie von Präzipitaten von C3N6, C3N12 und C3N18 untersucht, die nach einer Reaktionsdauer von zwanzig Minuten erhalten wurden (Daten nicht gezeigt). Hier gibt es keine auffälligen Unterschiede zu den Ansätzen, die zehn Minuten reagiert haben (siehe Abbildungen 4.2, 4.6 und 4.10). Bei Verwendung von C3N6 und C3N12 wurden kugelartige Silicastrukturen beobachtet, während mit C3N18 Kugeln und Filamente nebeneinander gebildet wurden. Sowohl was die Siliciummasse als auch die Struktur von präzipitiertem Silica betrifft, scheint dementsprechend eine Reaktionszeit von zehn Minuten ausreichend, um Aussagen über die polyamininduzierten $\mathrm{SiO}_{2}$-Präzipitationen machen zu können. 


\subsection{Diskussion}

Die Struktur und Entstehung von Diatomeenschalen wird auf drei Ebenen unterschieden. ${ }^{107}$ Die Mikroskala beschreibt die Gesamtgestalt, die in erster Linie von der Form des silica deposition vesicle (SDV) bestimmt wird. Auf der Nanoskala werden die initial auftretenden Polymerisationsprodukte betrachtet, die für die Ausbildung sehr kleiner Merkmale und Strukturen verantwortlich sind. In der Mesoskala werden Substrukturen im SDV gebildet, die zwischen Mikro- und Nanoskala liegen. ${ }^{108}$ Bezüglich der molekularen Vorgänge bei der Bildung der Diatomeenschalen auf der Nanoskala werden in der Literatur verschiedene Modelle diskutiert, anhand derer die Ausbildung der spezifischen Strukturen erklärt werden soll. Dabei spielen die Vorgänge im SDV eine wichtige Rolle. Während Gordon et al. ${ }^{29}$ in der diffusionslimitierten Aggregation von Silica-Partikeln die Grundlage für die Bildung der Mikrostrukturen sehen, konzentrieren sich Erklärungsansätze seit der Entdeckung der Silaffine und long chain polyamines (LCPA) überwiegend auf deren Einfluss. Vrieling et al. ${ }^{33}$ schlagen ein Modell vor, das auf der durch Silaffine und LCPAs dirigierten Aggregation von Silica-Nanopartikeln basiert. Die Entstehung von Poren wird hierbei durch die Anwesenheit großer organischer Moleküle erklärt, die strukturgebend sind und nach der Bildung des Silica aus der Struktur entfernt werden. Dahingegen stützt sich ein Modell von Sumper ${ }^{32,109}$ auf die Selbstorganisation der Silaffine und LCPAs zu Matrizen in Form von Nano- und Mikrotröpfchen, die bei der Silicabildung als Template dienen. Kröger ${ }^{66}$ geht darauf basierend von der Anordnung einer Silaffinmatrix aus, deren Gestalt überwiegend von der molekularen Struktur der Silaffine bestimmt wird und die durch Phasenseparationsprozesse entsteht. In dieser Matrix ordnen sich die LCPAs an und die Silicabildung wird in Regionen, in denen sie angereichert sind, induziert. In allen Fällen wird den Polyaminen, ihrer Interaktion mit anderen im SDV anwesenden Molekülen und Phasenseparationsprozessen eine große Bedeutung zugeschrieben. Insofern kann die systematische Untersuchung des Einflusses von Strukturmerkmalen der Polyamine und Reaktionsbedingungen auf Silicapräzipitationsreaktionen, wie sie im Rahmen dieser Arbeit durchgeführt wurde, zum Verständnis der Biomineralisation bei Diatomeen beitragen. Zu diesem Zweck wurden insgesamt sieben Polyamine untersucht, die sich in ihrer Kettenlänge, der Alkylgruppe zwischen den Aminoeinheiten beziehungsweise der $\mathrm{N}$-Methylierung unterscheiden. 


\section{Eigenschaften der Polyamine}

Um Zusammenhänge zwischen Molekülstruktur und Präzipitationsverhalten aufklären zu können, wurden zunächst einige Eigenschaften der Polyamine analysiert. Da vor allem der Akkumulation von positiven Ladungen eine wichtige Funktion zugeschrieben wird, ist das Säure-Base-Verhalten der Moleküle von besonderem Interesse. In Tabelle 4.2 sind verschiedene Informationen zu den eingesetzten Polyaminen zusammengefasst.

Tabelle 4.2 Eigenschaften der Polyamine C3N6, C3N12, C3N18, C2N7, C3N7, C4N7 und C3N12Me bei $\mathrm{pH} 6,8$.

\begin{tabular}{ccccccccc}
\hline Abkürzung & $\boldsymbol{N}_{\mathbf{N}}$ & $\boldsymbol{I} \boldsymbol{\AA} \AA$ & $\overline{\boldsymbol{I}}_{\boldsymbol{i}} \boldsymbol{I} \AA$ & $\mathbf{p K}_{\text {s Nprim }}$ & $\overline{\mathbf{p K}_{\mathbf{s}}}{ }_{\text {Nsek }}$ & $\overline{\boldsymbol{z}}$ & $\boldsymbol{z} \boldsymbol{l} \%$ & $\boldsymbol{h} \boldsymbol{I} \%$ \\
\hline C3N6 & 6 & 26,1 & 4,9 & 10,17 & 10,28 & 4,5 & 75 & 78,6 \\
\hline C3N12 & 12 & 54,6 & 4,9 & 10,17 & 10,27 & 8,4 & 70 & 81,3 \\
\hline C3N18 & 18 & 81,3 & 4,9 & 10,17 & 10,27 & 12,4 & 69 & 82,0 \\
\hline C2N7 & 7 & 23,3 & 3,7 & 9,86 & 9,69 & 3,9 & 56 & 79,4 \\
\hline C3N7 & 7 & 31,1 & 4,9 & 10,17 & 10,27 & 5,1 & 73 & 79,6 \\
\hline C4N7 & 7 & 39,7 & 6,4 & 10,43 & 10,69 & 6,8 & 97 & 78,1 \\
\hline C3N12Me & 12 & 56,4 & 5,1 & 9,86 & 9,42 & 7,3 & 61 & 86,7 \\
\hline
\end{tabular}

Neben Gesamtzahl der Aminogruppen $N_{\mathrm{N}}$ fasst Tabelle 4.2 Angaben zur Länge der Moleküle $l$ sowie zum mittleren Abstand zwischen zwei Aminoeinheiten $\bar{l}_{i}$ zusammen. Diese wurden mit Hilfe der Software ChemBio 3D Ultra abgeschätzt. Weiterhin wurden mit dem $\mathrm{pK}_{\mathrm{s}}$-Tool der Anwendung SPARC ${ }^{70}$ verschiedene Berechnungen durchgeführt. Zum einen können die $\mathrm{pK}_{\mathrm{s}}$-Werte der jeweils konjugierten Säure jeder Aminogruppe für den ersten Protonierungsschritt ermittelt werden. Im Fall der sekundären Gruppen unterscheiden sich die $\mathrm{pK}_{\mathrm{s}}$-Werte leicht, deshalb ist ein Mittelwert für alle angegeben. Zum anderen liefern makroskopische Analysen, bei denen auch weitere Protonierungsschritte mit in Betracht gezogen werden, jeweils die Verteilung von Molekülen unterschiedlicher Ladung bei verschiedenen $\mathrm{pH}-$ Werten. Mit diesen Daten konnte die mittlere Molekülladung $\bar{z}$ bei pH 6,8, bei dem die Experimente durchgeführt wurden und, daraus abgeleitet, der Protonierungsgrad $(z / \%)$ (tatsächliche Ladung/maximal mögliche Ladung) abgeschätzt werden. Allgemein muss beachtet werden, dass durch die SPARC-Berechnungen zwar die Gesamtmoleküle bei bestimmten pH-Werten betrachtet werden können, Einflüsse wie Ionenstärke des Puffers, Ionenart und Aggregation zwischen den Polyaminmolekülen können jedoch nicht berücksichtigt werden. Weiterhin wurde für die Hydrophobizität 
( $h / \%)$ der Moleküle eine einfache Abschätzung durchgeführt. Zu diesem Zweck wurden Kohlenstoffatome und ungeladene Stickstoffatome als hydrophob gewertet, positiv geladene Stickstoffatome wurden als hydrophil eingerechnet. Der Quotient aus vorliegender und maximal möglicher Hydrophobizität ergibt $h / \%$. Abbildung 4.27 veranschaulicht die in Tabelle 4.2 aufgeführten Parameter, vor allem der Unterschied in der Ladungsverteilung soll hierbei deutlich werden. Zu diesem Zweck sind Stickstoffatome mit positiver Formalladung rot dargestellt und ungeladene blau.

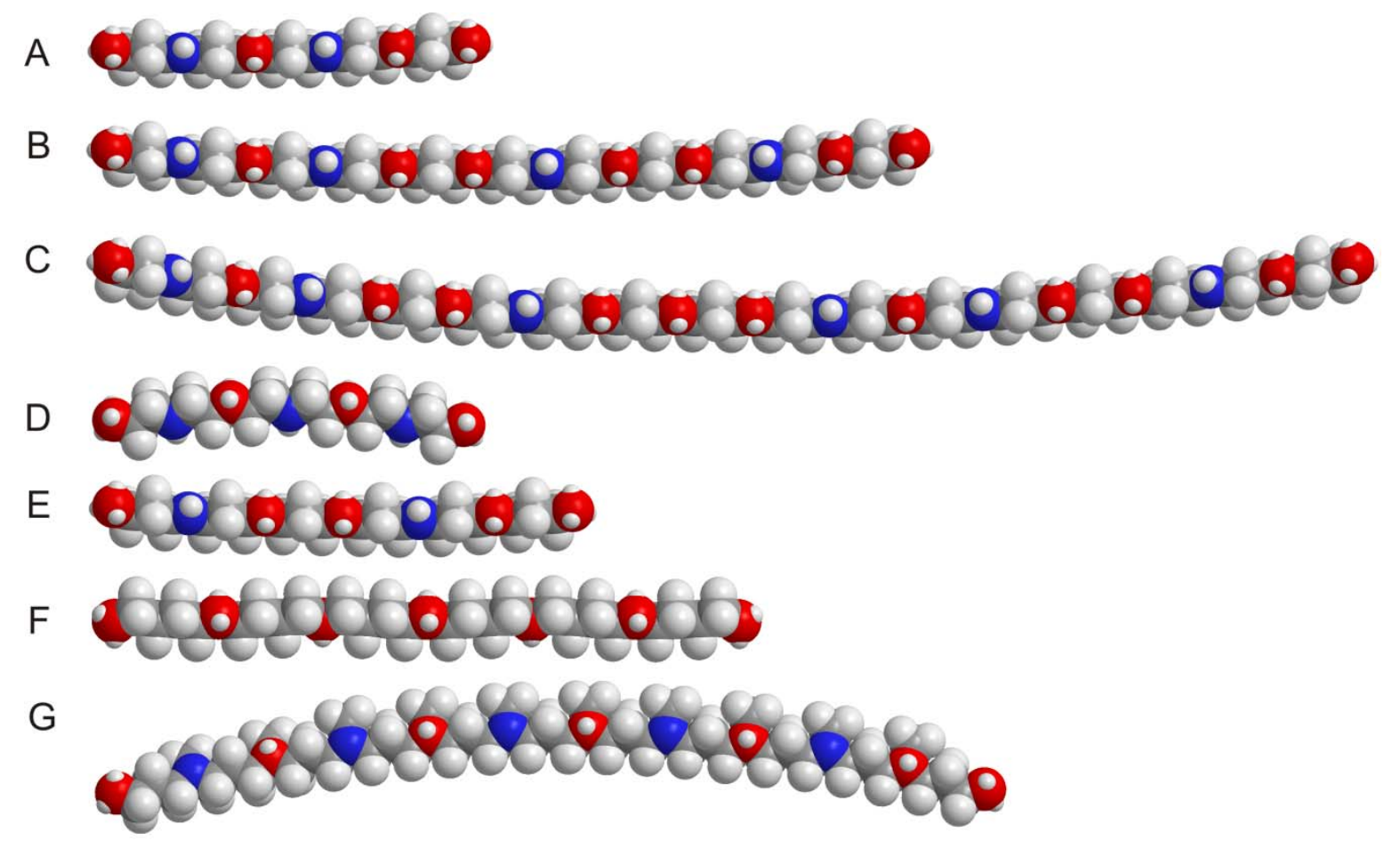

Abbildung 4.27 Kalottenmodelle der Polyamine A C3N6, B C3N12, C C3N18, D C2N7, E C3N7, F C4N7 und G C3N12Me mit wahrscheinlicher Ladungsverteilung bei pH 6,8. $\bigcirc \mathrm{C}, \bigcirc \mathrm{N}, \bigcirc \mathrm{N}^{+}, \mathcal{O} \circ \mathrm{H}$

Die Abstände zwischen den Stickstoffatomen sind aufgrund gleicher Alkylgruppen bei den Propylpolyaminen C3N6, C3N12 und C3N18 mit 4,9 A identisch. Damit im Zusammenhang stehend unterscheiden sich die $\mathrm{pK}_{\mathrm{s}}$-Werte ihrer primären beziehungsweise sekundären Aminogruppen nicht wesentlich, was sich auch in einem ähnlichen Protonierungsgrad äußert. Dieser nimmt mit zunehmender Kettenlänge geringfügig ab und liegt zwischen 75 und $69 \%$. Die hier aufgeführten Daten zum Säure-Base-Verhalten können nicht mit experimentellen aus der Literatur verglichen werden, da systematische potentiometrische Untersuchungen zum Einfluss der Kettenlänge auf die Basizität zwar mit kürzerkettigen Ethylpolyaminen (bis zu C2N7) durchgeführt wurden ${ }^{110}$, jedoch nicht mit 
längerkettigen Propylaminen. Bezüglich der Hydrophobizität gibt es zwischen den drei Verbindungen Unterschiede, sie ist für C3N18 am höchsten.

Beim Vergleich von C2N7, C3N7 und C4N7 zeigen sich nicht nur Abweichungen in den Gesamtmoleküllängen, auch der Abstand zwischen den Aminoeinheiten wird von C2 über C3 bis zu C4 größer und beträgt zwischen 3,7 $\AA$ und 6,4 $\AA$. Die $\mathrm{pK}_{\mathrm{s}}$-Werte aller Aminogruppen und damit der Protonierungsgrad nehmen analog deutlich zu. Während bei C2N7 bei pH 6,8 lediglich $56 \%$ der Aminogruppen positiv geladen sind, ist C4N7 fast vollständig protoniert (97\%). Die Tendenzen dieser theoretischen Daten stehen im Einklang mit experimentellen Befunden, bei denen kürzere Polyamine mit unterschiedlichen Alkylgruppen (C2N2-C6N2) untersucht wurden. ${ }^{11,112}$ Die Hydrophobizitäten der drei Polyamine unterscheiden sich nicht wesentlich.

In Verbindung C3N12Me ist der Abstand zwischen den Stickstoffatomen mit 5,1 $\AA$ etwas länger als in $\mathrm{C} 3 \mathrm{~N} 12$. Deutlichere Unterschiede finden sich in den $\mathrm{pK}_{\mathrm{s}}$-Werten, die bei C3N12Me niedriger sind, und damit in der Ladung und im Protonierungsgrad. Weist C3N12 eine mittlere Ladung von 8,4 und 70\% Protonierung auf, betragen diese Werte bei $\mathrm{C} 3 \mathrm{~N} 12 \mathrm{Me}$ 7,3 beziehungsweise $61 \%$. C3N12Me ist also im Vergleich zu C3N12 weniger basisch. Dieser Effekt, der durch Methylierung von Aminogruppen bewirkt wird, zeigte sich auch bei experimentellen potentiometrischen Untersuchungen. ${ }^{111}$ Die Hydrophobizität von C3N12Me ist, bedingt durch die geringe Gesamtladung und die zusätzlichen Methylgruppen, im Vergleich zu den anderen untersuchten Polyaminen am höchsten.

In diesem Zusammenhang ist es wichtig, zu klären, in welcher Form Kieselsäure beziehungsweise Silica bei den gewählten Reaktionsbedingungen vorliegt. Während monomere Kieselsäure $\mathrm{Si}(\mathrm{OH})_{4}$ einen $\mathrm{pK}_{\mathrm{s}}$-Wert von ca. 9,9 ausweist, liegt derjenige von höher polymerisierten, also kolloidalen Silicapartikeln bei ca. 6,7. ${ }^{35}$ Demnach liegen bei pH 7 ca. 1,8 \% der Silanolgruppen von Monokieselsäure als Silanolat vor, während unter gleichen Bedingungen $61 \%$ der Silanolgruppen von Silicapartikeln negativ geladen sind. ${ }^{113}$

\section{Unterschiede im Präzipitationsverhalten der Polyamine}

Beim Vergleich von Präzipitationsreaktionen, die durch Polyamine mit unterschiedlichen Strukturmerkmalen ausgelöst werden, konnten sowohl quantitative als auch morphologische Unterschiede ermittelt werden. Veränderungen der Molekülkettenlänge, der Alkylgruppe zwischen den Aminoeinheiten sowie die $N$-Methylierung beeinflussen zunächst die Aktivität bezüglich der Silicamenge, die bei den Reaktionen gebildet wird. 
Auch wenn alle zur Präzipitation verwendeten Polyaminlösungen ein festes N:SiVerhältnis von 1:100 besaßen, variierten die Siliciummassen des jeweils präzipitierten Silica im Bereich von 1,4 $\mu \mathrm{g}$ bis 30,5 $\mu \mathrm{g}$ (Abbildung 4.28).

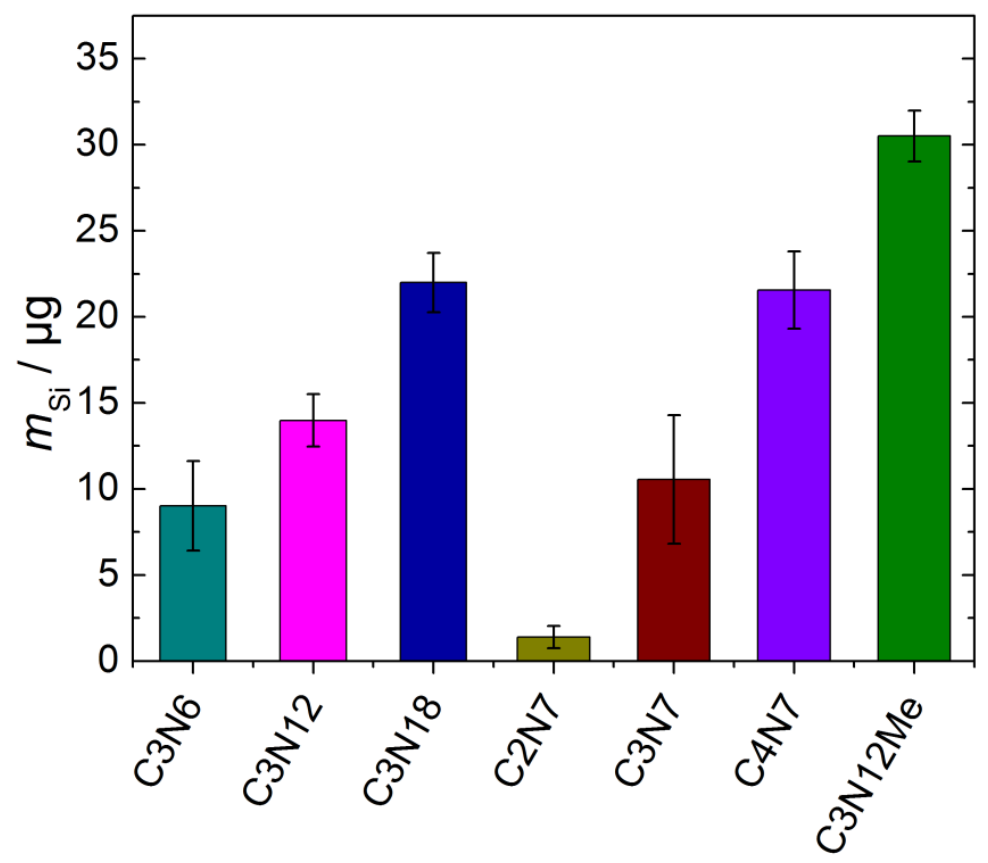

Abbildung 4.28 Überblick über die mittels $\beta$-Molybdatmethode bestimmten Massen $m_{\mathrm{Si}}$ an präzipitiertem Silicium für die Polyamine C3N6, C3N12, C3N18, C2N7, C3N7, C4N7 und C3N12Me. Reaktionsbedingungen: $30 \mathrm{~mm}$ Phosphatpuffer $\mathrm{pH}$ 6,8, $c_{\mathrm{N}}=1 \mathrm{mM}, c_{(\mathrm{SiOH})_{4}}=100 \mathrm{mM}$, Reaktionszeit: $10 \mathrm{~min}$.

Die Analyse der Durchmesser gebildeter Silicakugeln weist auf signifikante Größenänderungen in Abhängigkeit des verwendeten Polyamins hin. Abbildung 4.29 gibt einen Überblick über die Ergebnisse.
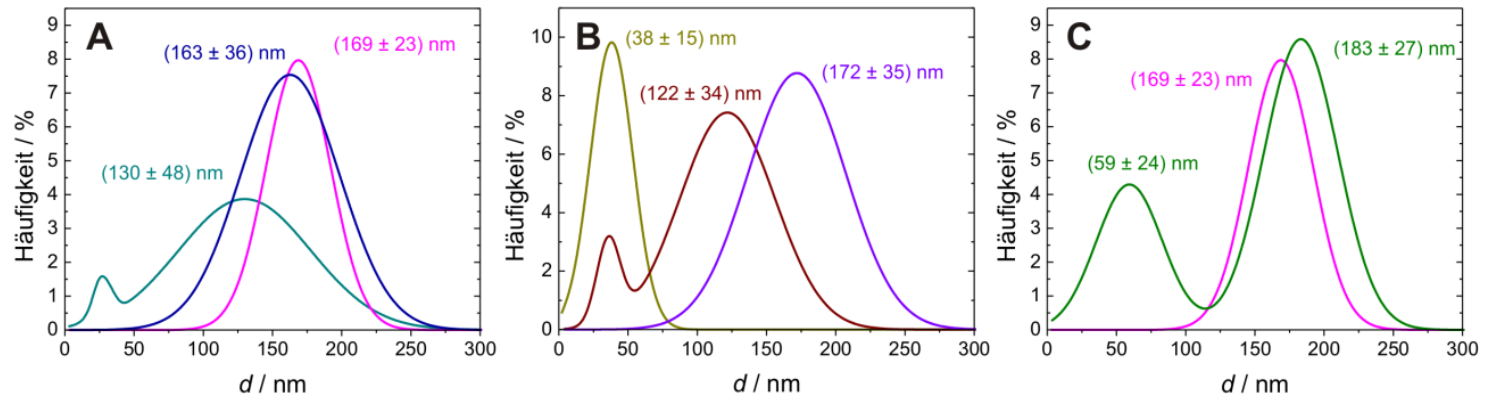

Abbildung 4.29 Verteilungen der Durchmesser der Silicapartikel aus Präzipitationen mit Lösungen von $\mathbf{A}-\mathrm{C} 3 \mathrm{~N} 6-\mathrm{C} 3 \mathrm{~N} 12-\mathrm{C} 3 \mathrm{~N} 18$ B $-\mathrm{C} 2 \mathrm{~N} 7-\mathrm{C} 3 \mathrm{~N} 7-\mathrm{C} 4 \mathrm{~N} 7$ und $\mathbf{C}-\mathrm{C} 3 \mathrm{~N} 12$ und $-\mathrm{C} 3 \mathrm{~N} 12 \mathrm{Me}$. Reaktionsbedingungen: $30 \mathrm{mM}$ Phosphatpuffer $\mathrm{pH} 6,8, c_{\mathrm{N}}=1 \mathrm{mM}, c_{(\mathrm{SiOH})_{4}}=100 \mathrm{mM}$, Reaktionszeit: $10 \mathrm{~min}$. 


\section{Der Einfluss der Kettenlänge}

Die Untersuchung der Silicapräzipitation mit Propylpolyaminen unterschiedlicher Kettenlänge lieferten folgende Erkenntnisse:

- C3N6, C3N12 und C3N18 präzipitieren ungleiche Silicamengen, die Zunahme von $m_{\mathrm{Si}}$ aufgetragen gegen die Kettenlänge erscheint dabei annähernd linear (Abbildung 4.28).

- Die Morphologie der Präzipitate von C3N6, C3N12 und C3N18 unterscheidet sich. Bei der Verwendung von C3N6 sowie C3N12 wurde ausschließlich kugelförmiges Silica gebildet. Bei Einsatz des Polyamins C3N18 kommen zusätzlich zu sphärischem Silica Filamente vor.

- Die Größen der auftretenden Silicakugeln unterscheiden sich für alle drei Polyamine signifikant voneinander. Abbildung 4.29 A gibt einen Überblick über die Verteilung der Durchmesser der gemessenen Silicakugeln.

Es stellt sich die Frage, welche Faktoren für die beschriebenen Unterschiede im Präzipitationsverhalten verantwortlich sind. Aufgrund der Komplexität der Polymerisation mit vielen aufeinanderfolgenden, teilweise reversiblen Reaktionsschritten ist es schwer zu beantworten, auf welcher Ebene Polyamine die Silicabildung beeinflussen. Wird die Kondensationsreaktion katalysiert, wird die Aggregation von Solpartikeln oder diejenige größerer Partikel beeinflusst? Oder stellt die Phasenseparation der Polyamine den entscheidenden Schritt dar? In der Literatur finden sich unterschiedliche Hypothesen.
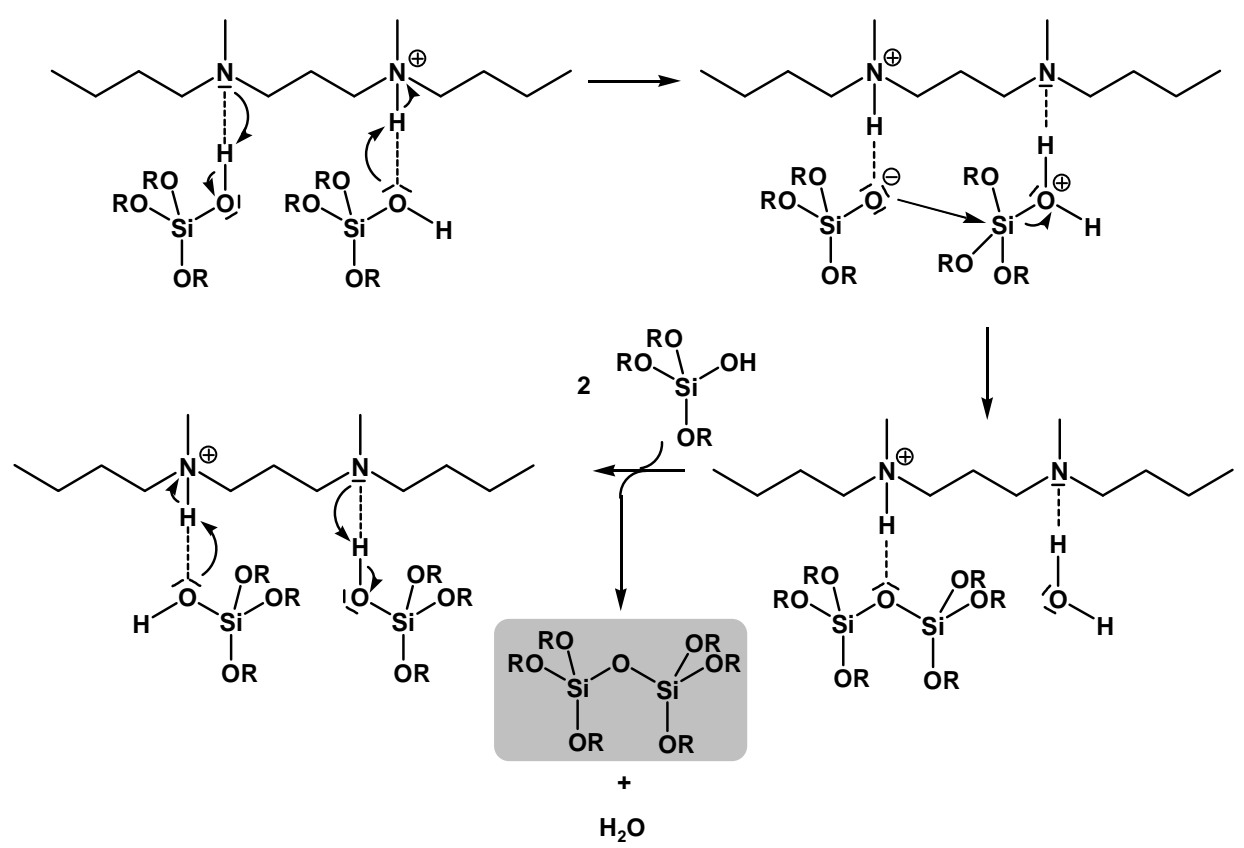

Abbildung 4.30 Von Kröger ${ }^{114}$ vorgeschlagener Mechanismus der Bildung einer Siloxanbindung, katalysiert durch einen Abschnitt eines $N$-Methyl-Propylamins beziehungsweise einer Silaffinseitenkette. 
So postulierten Mizutani et al., ${ }^{42}$ dass Aminogruppen mit geeignetem Abstand den Übergangszustand der Kondensationsreaktion stabilisieren können. Kröger ${ }^{114}$ schlug einen Mechanismus vor, nach dem Polyamine direkt an der Bildung von Siloxanbindungen beteiligt sind (Abbildung 4.30). Die Position der Aminogruppen im jeweiligen Molekül sowie ein Nebeneinander von geladenen und ungeladenen Aminogruppen, welche durch günstige Anordnung die Kondensationsreaktion unterstützen sollen, ist bei beiden von Bedeutung. Da der Abstand zwischen den Aminogruppen in den drei Propylaminen jeweils gleich groß ist (Tabelle 4.2, Abbildung 4.27), kann dieser Faktor hier keine direkte Rolle spielen.

Belton et al. ${ }^{102}$ beschreiben eine Abhängigkeit, nach der die Ratenkonstante der Bildung des Kieselsäuretrimers mit der Kettenlänge von Polyaminen ansteigt, was auf einen Einfluss von Polyaminen auf die ersten Kondensationsschritte schließen lässt. Dies stimmt mit den Beobachtungen aus weiteren Veröffentlichungen überein, nach denen jeweils zunehmende Kettenlängen von Polyaminen oder auch Polypeptiden schnellere oder vermehrte Silicabildung bewirken. ${ }^{42-44} \mathrm{Da}$ in der vorliegenden Arbeit die Endprodukte analysiert wurden, kann über die ersten Reaktionsschritte keine Aussage getroffen werden. Der Trend ist jedoch der gleiche, mit der Polyaminkettenlänge steigt die präzipitierte Siliciummasse. Der Effekt wird zum Beispiel von Coradin et al. ${ }^{44}$ auf eine erhöhte Anzahl an $\mathrm{NH}_{3}{ }^{+}-$ Bindungsstellen zurückgeführt. Allerdings muss bedacht werden, dass im Fall von C3N6, C3N12 und C3N18 in jedem Reaktionsansatz die Gesamtzahl an Aminogruppen identisch war und auch die Anzahl protonierter Gruppen in diesem Fall aufgrund der ähnlichen Protonierung (Tabelle 4.2, Abbildung 4.27) fast gleich ist.

Auf später ablaufende Schritte während der Silicabildung bezogen sich andere Arbeiten. Laut Behrens et al. ${ }^{115}$ haben unterschiedliche Kettenlängen von Polyaminen keinen katalytischen Effekt auf die ersten Schritte der Kondensation, sondern ausschließlich auf anschließende Aggregationsprozesse. Iler ${ }^{35}$ beschreibt, dass Polymere die Silicabildung umso stärker induzieren, je länger sie sind, mit der Begründung, dass sie dann in größerem Umfang Silicapartikel vernetzen können, was bei der Präzipitation im Gegensatz zum Gelprozess essenziell ist. So spielt dieser Effekt der Kettenverlängerung auf die Polymerisation erst ab einer Molekülmasse von $1000 \mathrm{~g} / \mathrm{mol}$ keine Rolle mehr. ${ }^{35} \mathrm{C} 3 \mathrm{~N} 18$ hat eine Molekülmasse von 987,6 g/mol, was dafür spricht, dass bei den drei Propylaminen die Kettenlänge für die Unterschiede in den präzipitierten Mengen mitverantwortlich ist. Auch Coradin $^{44}$ sah hierin unter anderem einen Erklärungsansatz, er beschrieb einen kooperativen Prozess, durch den Kieselsäureeinheiten entlang von Peptidketten in 
räumliche Nähe gebracht werden, wodurch ihre Kondensation erleichtert wird. Iler jedoch ging davon aus, dass einzelne Polyamine auf den negativ geladenen Oberflächen von Silicaoligomerpartikeln adsorbieren und so deren Verbrückung bewirken. Hingegen beobachteten Kröger et al. ${ }^{31}$ beziehungsweise Sumper et al. ${ }^{41,65}$ in vitro mit Silaffinen beziehungsweise Polyaminen Phasenseparationsprozesse, wobei sich diese amphiphilen Moleküle zu Mikrotröpfchen zusammenlagern sollen und die Silicabildung an der Grenzfläche stattfindet. Dies würde einen anderen Prozess als den von Iler beschriebenen darstellen und der oben beschriebene direkte Einfluss der Kettenlänge auf die Verbrückung von Silica ist hierbei schwerer abzuschätzen. Belton et al. gehen davon aus, dass die Bildung supramolekularer Anordnungen der Polyamine von ihren Strukturen und Eigenschaften abhängt und diese Aggregatbildung wiederum Präzipitationsgeschwindigkeit und -menge beeinflusst. ${ }^{102}$ Aus Tabelle 4.2 geht hervor, dass von C3N6 über C3N12 bis zu C3N18 der Anteil an protonierten Spezies abnimmt (75\% - 69\%), wenngleich die Änderung nicht groß ist. In der gleichen Reihenfolge nimmt die Hydrophobizität zu, während der Abstand zwischen den einzelnen Aminoeinheiten konstant ist. Diese beiden Effekte bedeuten geringere elektrostatische Repulsion zwischen den Molekülen bei höherem Polymerisationsgrad, was die Aggregation begünstigen sollte und damit eine Erklärung für das erhöhte Präzipitationspotenzial darstellen könnte.

\section{Der Einfluss der Kettenlänge auf die Morphologie}

Bezüglich der Morphologie des gebildeten Silica könnten diese Parameter, wenn sie die Aggregation beeinflussen, auch die unterschiedlichen Durchmesser der Kugeln erklären (Abbildung 4.29). Geht man davon aus, dass die Silicakondensation an der Grenzfläche zwischen Polyamin und Wasser stattfindet, ${ }^{32,116}$ wäre es von großer Bedeutung, die Gestalt der Polyaminaggregate vor der Kieselsäurezugabe zu kennen. Nicht gezeigte Lichtstreuungsmessungen belegen, dass alle Polyamine Aggregate mit Durchmessern im Bereich von $(100-1000) \mathrm{nm}$ in $30 \mathrm{mM}$ Phosphatpuffer pH 6,0 bildeten. Die jeweiligen genauen Größen konnten dabei nicht reproduzierbar bestimmt werden. Es kann jedoch davon ausgegangen werden, dass aufgrund der unterschiedlichen attraktiven beziehungsweise repulsiven Kräfte die Gestalt der Polyaminaggregate durch die Kettenlänge beeinflusst wird. Lenoci et al. ${ }^{117}$ schlugen zwei Szenarien für die Bildung von SilicaPeptid-Kompositen vor, die nun auch auf die Polyamine übertragen werden sollen. Sie gehen davon aus, dass bei Peptidvolumenanteilen unter $5 \%$ Aggregate vorliegen, an deren Oberfläche die Silicabildung induziert und von dort aus nach außen hin fortgesetzt wird. 
Bei Volumenanteilen über $5 \%$ hingegen soll ein Peptidnetzwerk das Wachstum von Silica begrenzen und dirigieren. Sie postulieren, dass im ersten Fall das Peptid in den Kernen von Silicapartikeln angereichert ist, während es in zweitem Fall eher gleichmäßig verteilt sein müsste. ${ }^{117}$ Diese Effekte könnten nicht nur durch die Konzentration bedingt werden, sondern im Fall der Polyamine auch durch deren Strukturen und Eigenschaften, die die Aggregation beeinflussen. Interessant ist, dass im Fall von C3N6 hohle $\mathrm{SiO}_{2}$-Kugeln vorliegen, in deren Schalen auch Polyamin zu finden ist, manche dieser Kugeln aber mit organischem Material gefüllt sind, was aus den EDX-Messung deutlich wird (Abbildungen 4.4 und 4.5). Dies entspricht dem Szenario bei geringer Peptidkonzentration von Lenoci et al. Bei C3N12 und C3N18 sind solche Anreicherungen im Inneren von Partikeln nicht $\mathrm{zu}$ finden (Abbildungen 4.8, 4.9, 4.12 und 4.13). Murthy et al. ${ }^{18}$ konnten bei Experimenten mit Poly(L-Lysin) beziehungsweise Polyallylamin visualisieren, dass nach Zugabe von Citrat-Anionen beide Substanzen in wässriger Lösung phasenseparieren und runde Aggregate gebildet werden, die im Fall von Poly(L-Lysin) größer sind als bei Polyallylamin. Bei anschließender Zugabe von $\mathrm{SiO}_{2}$-Nanopartikeln entstanden jeweils Silicakügelchen, die im SEM massiv erscheinen. Konfokalmikroskopische Aufnahmen zeigten jedoch, dass Poly(L-Lysin) ausschließlich im Schalenbereich der hohlen, mit Wasser gefüllten Kugeln angereichert ist $^{119}$, während sich Polyallylamin sowohl in den Schalen als auch im Inneren findet ${ }^{120}$. Es ist nicht geklärt, wodurch dieser Unterschied zustande kommt, könnte aber in den Eigenschaften und damit dem Aggregationsverhalten der Polymere begründet liegen. Das Auftreten der Filamente mit Polyamin C3N18 gibt einen weiteren Hinweis darauf, dass die Polyamin-Phosphat-Aggregation von der Struktur und den Eigenschaften der Polyamine abhängt. Ähnliche Silicastrukturen werden beispielsweise dann beobachtet, wenn bei der durch Polyallylamin induzierten Bildung externe Scherkraft angelegt wird ${ }^{121}$ beziehungsweise wenn Präzipitation mittels Poly(LLysin) unter Einfluss eines elektrostatischen Feldes durchgeführt wird ${ }^{122}$. Die Verwendung von Hydrogelen mit silicapräzipitierenden Eigenschaften führte ebenfalls zur Ausbildung von Silicafasern. ${ }^{123-126}$ Van Bommel et al. ${ }^{127}$ beobachteten dabei nicht nur fadenförmiges Silica, sondern auch solches, bei dem Silicakugeln wie auf einer Kette aufgereiht und teilweise miteinander verschmolzen sind. Sie erinnern an Strukturen, die stellenweise mit C3N18 beobachtet wurden (Abbildungen 4.10 D und 4.11 A). Sie erklären ihre Bildung über das Wachstum von Silicakugeln entlang von Organogelfasern, die mit zunehmender Größe miteinander verschmelzen können. Die Durchmesser der Silicafilamente, die mit C3N18 gebildet wurden, liegen bei $33 \mathrm{~nm}$, was ungefähr dem Durchmesser der kleinsten 
auftretenden Kugeln entspricht, was einen Beleg für diese Hypothese darstellt. Weiter ist interessant, dass die Filamente keine Hohlräume aufzuweisen scheinen (Abbildungen 4.11 und 4.12). Laut Belton et al. ${ }^{102}$ sind Silicastrukturen dann hohl, wenn die Amine sich zu Tröpfchen zusammenlagern, was bei allen Silicakugeln in der vorliegenden Arbeit beobachtet wurde. Im Fall der Filamente bei C3N18 könnten demnach vor der Kieselsäurezugabe andere Polyaminaggregate neben Tröpfchen vorgelegen haben. Das könnte auch erklären, warum die mit C3N12 gebildeten Kugeln etwas größere Durchmesser, als die mit C3N18 auftretenden, hatten. Eventuell konkurriert ab einer gewissen Kettenlänge die Bildung von Polyaminmikrotröpfchen mit der Entstehung von faserigen Strukturen, was die Tröpfchengröße begrenzt. Der Vergleich der Durchmesser der Kugeln verdeutlicht, dass die mit C3N6 gebildeten kleiner sind und die Verteilung ihrer Durchmesser sehr viel breiter ist als der mit C3N12 gebildeten. Dies kann als Hinweis darauf gedeutet werden, dass C3N12 gleichmäßiger aggregiert und somit die Struktur der Polyamine das Phasenseparationsverhalten beeinflusst. Die Tatsache, dass die Silicakugeln bei den drei Polyaminen eng miteinander verbunden sind, kann dadurch erklärt werden, dass diese Anordnung in allen Fällen die niedrigste freie Energie aufweist. ${ }^{108}$

In den Experimenten wurde nachgewiesen, dass die Erhöhung der Polyaminkonzentration von $c_{\mathrm{N}}=1 \mathrm{mM}$ auf $2 \mathrm{mM}$ eine Vergrößerung der präzipitierten Siliciummasse bewirkt (Abbildung 4.14), während eine Reduktion der Kieselsäurekonzentration von $100 \mathrm{~mm}$ auf $40 \mathrm{mM}$ keine Veränderung bewirkte. Dies lässt darauf schließen, dass mit einem Überschuss an $\mathrm{Si}(\mathrm{OH})_{4}$ gearbeitet wurde. Die Abhängigkeit der Silicamenge von der Konzentration der eingesetzten Amine oder Peptide ist bekannt. ${ }^{50,103,106}$ In (nicht gezeigten) Untersuchungen zeigte sich, dass auch die Morphologie der Silicastrukturen von der Polyaminkonzentration abhängt. Durchschnittlich stieg die Kugelgröße durch Konzentrationserhöhung an, ein Phänomen, das auch Patwardhan beobachtete. ${ }^{128}$ Bei C3N12 trat beispielsweise bei einer Konzentration von $c_{\mathrm{N}}=2,4 \mathrm{mM}$ auch filamentartiges Silica auf, wie das bei C3N18 $\left(c_{\mathrm{N}}=1 \mathrm{mM}\right)$ der Fall war und die Strukturen waren weniger voneinander separiert. Diese Erkenntnisse fügen sich in das vorher diskutierte Bild. Eine Konzentrationserhöhung könnte eine andere Packung zur Folge haben und dadurch die beschriebenen Effekte hervorrufen. Die Phasenseparationsprozesse hätten demnach einen entscheidenden Einfluss auf die Silicapräzipitation. 


\section{Der Einfluss der Alkylgruppe der Polyamine}

Beim Vergleich von Polyaminen mit unterschiedlichen Alkylgruppen zwischen den Aminoeinheiten (C2N7, C3N7, C4N7) bezüglich ihrer $\mathrm{SiO}_{2}$-präzipitierenden Funktion zeichneten sich mehrere Unterschiede ab:

- Im Fall von C2N7, C3N7 und C4N7 bewirkt eine Verlängerung der Alkylgruppe von Ethyl- über Propyl- bis zu Butyleinheit eine nahezu lineare Zunahme der Si-Menge (Abbildung 4.28).

- Mit Verlängerung der hydrophoben Gruppe werden die gebildeten Silicakugeln größer (Abbildung 4.29 B).

- Mit allen drei Verbindungen werden ausschließlich Kugeln gebildet (Abbildungen 4.16 - 4.18), filamentartiges $\mathrm{SiO}_{2}$ wie bei C3N18 tritt nicht auf.

Auch für diese Versuchsreihe gilt es zu klären, wodurch die beobachteten Unterschiede verursacht werden können. Wiederum sollte unterschieden werden, ob die Polyamine katalytische Funktion bei der Kondensationsreaktion haben und/oder die später ablaufende Aggregation bedingen. Der Vergleich der Eigenschaften der drei Verbindungen verdeutlicht, dass anders als bei C3N6-C3N18 sich die Hydrophobizität kaum unterscheidet, jedoch der Protonierungsgrad stark variiert. Während bei pH 6,8 nur knapp mehr als die Hälfte $(56 \%)$ der C2N7-Moleküle positiv geladen sind, sind es bei C3N7 annähernd drei Viertel (73 \%), wohingegen die C4N7-Moleküle fast vollständig protoniert sind (97\%). Zudem ist der jeweilige Abstand zwischen zwei Aminogruppen unterschiedlich (Tabelle 4.2, Abbildung 4.27). Mizutani ${ }^{42}$ und Kröger ${ }^{114}$ postulierten, dass die Position der Aminogruppen von Bedeutung für eine katalytische beziehungsweise stabilisierende Funktion bei der Silicakondensation ist. Dieser Aspekt scheint sich hier auszuwirken, da bei Vorhandensein einer Ethylgruppe (C2N7) die Präzipitation in geringem Ausmaß stattfand. Die Verlängerung zu Propyl- beziehungsweise Butyleinheiten bewirkte hingegen eine Erhöhung der detektierten Silicamenge (siehe Abbildung 4.32). Die stark unterschiedliche Protonierung der drei Moleküle kann jedoch einen mindestens genauso großen Einfluss wie der räumliche Abstand haben. In einigen Veröffentlichungen wird postuliert, dass die Anwesenheit von ungeladenen Aminogruppen mit freiem Elektronenpaar zur Ausbildung von Wasserstoffbrückenbindungen neben geladenen Aminoeinheiten für die Kondensationsreaktion und die Phasenseparation der Polyamine essenziell ist ${ }^{105,114}$ beziehungsweise sich förderlich auswirkt. ${ }^{35,44,105}$ Die vorliegende Untersuchung scheint diese Aussage zunächst nicht zu bestätigen, da C4N7 sehr großes Präzipitationspotenzial bei 
vollständiger Protonierung aufweist. Jedoch darf nicht außer Acht gelassen werden, dass in der durchgeführten Berechnung zum Protonierungszustand die Anwesenheit von Phosphatanionen und die Aggregation der Polyamine nicht berücksichtigt wurden. Es ist wahrscheinlich, dass durch räumliche Nähe die tatsächlichen $\mathrm{pK}_{\mathrm{s}}$-Werte niedriger sind als die theoretischen. Dadurch bedingt wäre die Protonierung verringert, was dazu führen könnte, dass im Fall von C4N7 eine größere Menge Silica präzipitiert, weil dann auch Aminogruppen mit freiem Elektronenpaar anwesend wären. Bei C2N7 hingegen könnte eine zu geringe Ladungsakkumulation das Resultat sein. Betrachtet man die Eigenschaften der Polyamine bezüglich ihrer Silicapartikel verbrückenden Funktion, so kann die Kettenlänge beziehungsweise die Anzahl der Aminogruppen pro Molekül, wie sie bei C3N6-C3N18 diskutiert wurde, kein entscheidender Faktor sein. Die erhöhte Zahl an positiven Ladungen in C4N7 könnte eine für die elektrostatische Interaktion mit negativ geladenen Silicapartikeln günstige Rolle spielen. Zudem könnte die Verlängerung der Alkylgruppe zwischen den Aminogruppen eine bessere Flexibilität erlauben, wenn es darum geht, dass mehrere Aminogruppen eines Moleküls mit einem Silicapartikel interagieren müssen, was laut Iler ${ }^{35}$ nötig ist, um Präzipitation zu induzieren. Bezüglich der Phasenseparation liegen auch hier keine direkten Beobachtungen vor. Es ist jedoch vorstellbar, dass die Polyamine umso besser durch Phosphat verbrückt werden können, je höher sie geladen sind und je flexibler zwei nebeneinanderliegende Aminogruppen miteinander verbunden sind. Daraus kann gefolgert werden, dass die Aggregation von C4N7 über C3N7 bis C2N7 schwächer würde. Dies wiederum könnte das verringerte Präzipitationspotenzial erklären. Es kann nicht eindeutig differenziert werden, ob die Länge der Alkyleinheit, der Protonierungsgrad oder beide Faktoren zusammen ausschlaggebend sind.

Auffällig ist, dass C4N7 zwar eine ähnliche Menge Silicium präzipitiert wie C3N18, hierbei aber ausschließlich Silicakugeln auftreten. Die Bildung der Filamente scheint demnach ausschließlich von der Kettenlänge beziehungsweise der Zahl der Aminogruppen pro Molekül abzuhängen, während die Präzipitationsmenge durch verschiedene Faktoren beeinflusst wird. Die Zunahme des Kugeldurchmessers von C2N7 bis C4N7 (Abbildung 4.29 B) deutet, wie die Erhöhung der präzipitierten Siliciummasse, auf verstärkte Aggregation hin. TEM-Aufnahmen sowie EDX-Daten könnten auch für diese Versuchsreihe weitere Informationen über das Innere der Kugeln liefern. 


\section{Der Einfluss der $N$-Methylierung}

Als weiterer Aspekt wurde der Einfluss der $N$-Methylierung auf die Präzipitationseigenschaften untersucht. Hierzu wurden die Polyamine C3N12 und C3N12Me miteinander verglichen. Die Methylierung der sekundären Aminogruppen bei ansonsten identischer Struktur bewirkte eine ungefähre Verdoppelung der präzipitierten Siliciummasse (Abbildung 4.28). Gleichzeitig veränderte sich die Morphologie dahin gehend, dass statt einer Kugelpopulation wie bei C3N12 zwei auftreten. Eine Population wies größere Kugeldurchmesser auf, als die mit C3N12 gebildeten Silicakugeln, die kleinere besitzt deutlich geringere Durchmesser (Abbildung 4.29 C).

Robinson et al. ${ }^{129}$ und Belton et al. ${ }^{17}$ stellten fest, dass die Präzipitationsrate mit dem Methylierungsgrad ansteigt. Robinson et al. begründeten dies damit, dass die Alkylierung von Aminogruppen ihre Wechselwirkungen mit dem Lösungsmittel verringert und dadurch die Interaktion mit Silicaoligomeren verstärkt wird. Sie erachteten das Säure-BaseVerhalten als weniger wichtig, jedoch verglichen sie jeweils nur die $\mathrm{pK}_{\mathrm{s}}-$ Werte der ersten Protonierung der Verbindungen mit unterschiedlichen $N$-Methylierungen miteinander. Der Gesamtprotonierungszustand wurde dabei vernachlässigt, was zu verfälschten Schlussfolgerungen geführt haben könnte. Einen umgekehrten Trend in Bezug auf die Auswirkung der $N$-Methylierung auf die Präzipitation ermittelten zunächst Menzel und Behrens ${ }^{130}$, später führten sie diese Ergebnisse jedoch auf die Abwesenheit von Puffer und die daraus resultierenden unterschiedlichenn $\mathrm{pH}$-Werte zurück, die beim Lösen der Polyamine auftraten. ${ }^{115}$ Interessant ist der Vergleich des durch die $N$-Methylierung bewirkten Effekts mit dem durch die Kettenverlängerung beobachteten. Beide Fälle lieferten einen identischen Zusammenhang zwischen Moleküleigenschaften und Präzipitation. C3N12Me ist bei etwas geringerer Protonierung deutlich hydrophober als C3N12. Dies ist auch für C3N18 im Vergleich zu C3N12 der Fall, wenn auch etwas weniger deutlich ausgeprägt. Bei beiden Verbindungen sollten die elektrostatische Repulsion vermindert und zugleich attraktive Kräfte größer sein, was die Aggregation begünstigen sollte. Sowohl C3N12Me als auch C3N18 haben höheres Präzipitationspotenzial als C3N12. Diese Erkenntnisse stützen die Hypothese, dass Phasenseparation und Aggregation entscheidende Faktoren für Präzipitationsreaktionen sind. Die geringere Protonierung von C3N12Me bewirkt keine Abnahme der gebildeten Silicamenge, wie es bei der Reihe C2N7 bis C4N7 auffällig war. Auch im Fall des $N$-methylierten Polyamins C3N12Me könnten TEM- und EDXMessungen weitere Informationen zur Morphologie der Silicakugeln liefern. Es ist interessant, dass die Veränderung der $N$-Methylierung bei ansonsten identischen Struktur- 
merkmalen einen so drastischen Einfluss hat, wie durch den Vergleich von C3N12 mit C3N12Me aufgezeigt wurde.

\section{Der Einfluss der Phosphatkonzentration}

In verschiedenen Arbeiten wurde ermittelt, dass die Anwesenheit multivalenter Anionen unerlässlich für in vitro polyamininduzierte Präzipitationen ist. ${ }^{31,41,65,105}$ Mizutani et al. $^{42}$ gingen zwar nicht auf die Rolle von Anionen ein, doch auch bei den von ihnen beschriebenen Experimenten waren multivalente Anionen in Form von Borat anwesend. In anderen Studien, die ohne Anionen wie Phosphat durchgeführt wurden, liefen die Experimente auf einer längeren Zeitskala $\mathrm{ab}^{44,102}$ beziehungsweise konnte die Akkumulation von Ladung durch die Molekülstruktur realisiert werden ${ }^{106}$. In den Messungen der vorliegenden Arbeit wurde ebenfalls eine starke Abhängigkeit der Präzipitation von Phosphationen bei Verwendung der Polyamine C3N6, C3N12 und C3N18 gezeigt. Zum einen hängt die Menge an entstehendem Silica von der Anionenkonzentration ab (Abbildungen 4.12 und 4.22), zum anderen wird seine Struktur darüber reguliert (Abbildungen 4.23 - 4.25). Es wurde ermittelt, dass die Kettenlänge der Propylpolyamine einen Einfluss auf die Wechselwirkung mit den Phosphatanionen hat. Je länger die Kette, desto steiler war der Anstieg der Siliciummasse mit Zunahme der Anionenkonzentration und desto weniger Phosphat war nötig, um die maximal mögliche Präzipitation zu induzieren. Ab einem bestimmten Stickstoff-Phosphat-Verhältnis blieb die Siliciummasse auch bei weiterer Erhöhung der Phosphationenkonzentration konstant. Eine ähnliche Abhängigkeit konnten Brunner et al. ${ }^{41}$ beobachten. Anders als bei ihrer Untersuchung war in der vorliegenden Arbeit ein größeres $\mathrm{P}_{\mathrm{i}}: \mathrm{N}-$ Verhältnis nötig, um die maximale Präzipitation herbeizuführen, beispielsweise im Fall von C3N18 2,7:1, während Brunner einen Wert von ca. 0,6:1 ermittelte. Jedoch wurde dort Polyallylamin mit ca. 160 Aminoeinheiten pro Molekül verwendet. Setzt sich der oben erläuterte Trend fort, laut dem mit zunehmender Kettenlänge weniger Phosphat benötigt wird, so erscheint die Erhöhung des $\mathrm{P}_{\mathrm{i}}: \mathrm{N}-$ Verhältnisses sinnvoll zu sein. Es stellt sich die Frage, auf welchen Faktoren dieser Effekt beruht. Ruft man sich nochmals die Säure-Base-Eigenschaften sowie die Hydrophobizität der drei Propylpolyamine ins Gedächtnis (Tabelle 4.2), so fällt auf, dass für C3N18 die elektrostatische Repulsion am geringsten und die attraktiven Wechselwirkungen aufgrund der hydrophoben Eigenschaften am größten sein dürften. Das würde erklären, warum weniger verbrückendes Phosphat für die Aggregation nötig ist. Die positive Ladung von C3N18 ist zwar im Vergleich zu C3N6 sowie C3N12 am kleinsten, 
jedoch zeigten andere Arbeiten, dass die Phosphat-Polyamin-Komplexbildung beziehungsweise -Aggregation nicht ausschließlich auf elektrostatischen Interaktionen basieren, sondern dass auch Wasserstoffbrückenbindungen beteiligt sein müssen. ${ }^{105,131}$ In Kapitel 4.3 wurden SEM-Aufnahmen aufgeführt, die den Einfluss der Anionenkonzentration auf die Morphologie des Silica aufzeigen. Bei der Verwendung von C3N6 und $3 \mathrm{~mm}$ Phosphat, also im für dieses System phosphatabhängigen Bereich der Präzipitation (Abbildung 4.22) wurden schlecht definierte Silicastrukturen gebildet. Neben Kugeln, deren Durchmesser breit verteilt sind, findet sich sehr viel amorphes Material (Abbildung $4.23 \mathrm{~A}$ ), was auf unzureichende Aggregation der Polyamine hindeutet. Die Kugelgröße nimmt mit Erhöhung der Anionenkonzentration zu (Abbildung 4.31). Im Fall von C3N12 steigen die Kugeldurchmesser ebenfalls leicht an.
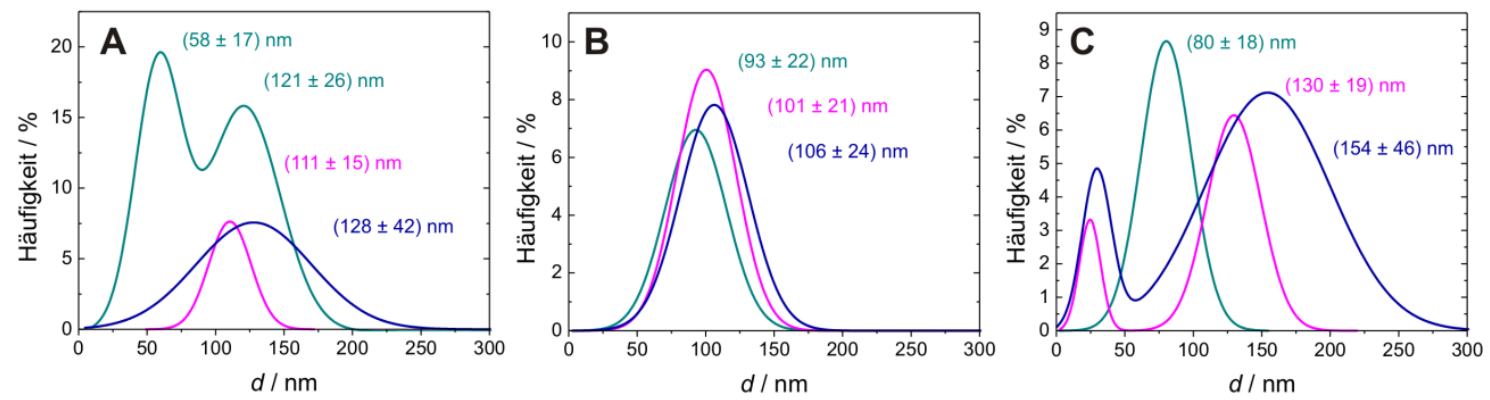

Abbildung 4.31 Verteilungen der Durchmesser der Silicapartikel aus Präzipitationen mit Lösungen von A C3N6, B C3N12, C C3N18 in Anwesenheit von $-3 \mathrm{~mm}=10 \mathrm{~mm}-30 \mathrm{~mm}$ Phosphationen.

Der Anstieg der Kugelgröße mit der Phosphatkonzentration wurde auch in anderen Arbeiten beobachtet. ${ }^{65,106}$ Dabei konnte von Brunner et al. ${ }^{41}$ mittels dynamischer Lichtstreuung eine analoge Vergrößerung von Polyaminaggregaten gezeigt werden, worin sie den entscheidenden Faktor für die Vergrößerung der Silicapräzipitate sehen. Genau wie eine Verlängerung der Polyaminkette oder die $N$-Methylierung bewirkt die Anwesenheit von Anionen eine Verstärkung der Aggregationsprozesse. Drastische Auswirkung hat die Konzentration der Phosphationen auf das System C3N18. Obwohl die Siliciummassen im Bereich $c_{\mathrm{Pi}}=3-10 \mathrm{mM}$ konstant ist, ändert sich das Aussehen des Silica auffällig. Liegen bei $3 \mathrm{mM}$ ausschließlich Kugeln vor, treten bei $10 \mathrm{mM}$ Filamente auf, welche bei $30 \mathrm{mM}$ noch häufiger sind. Die Größe der Kugeln nimmt wie bei C3N12 mit der Anionenkonzentration zu. Die Erhöhung der Phosphatkonzentration hat hier den gleichen Effekt, wie die Kettenverlängerung der Polyamine bei konstanter Anionenkonzentration. Diese Beobachtungen lassen den Schluss zu, dass der Überschuss an Anionen die Bildung anderer Polyaminaggregate neben Tröpfchen bewirkt. Eventuell gibt es faserförmige 
Anordnungen, entlang derer, wie oben beschrieben wurde, die Silicabildung stattfindet.

Die detektierten Abhängigkeiten von der Phosphatkonzentration fügen sich gut in das diskutierte Bild, laut dem die Aggregation und Phasenseparation der Polyamine einen entscheidenden Einfluss auf die Präzipitation von Kieselsäure haben müssen.

\section{Zusammenfassung}

Um die bereits erläuterten Trends besser einordnen zu können, wurden in einem Diagramm die präzipitierten Siliciummassen gegen den Protonierungsgrad und die Hydrophobizität aufgetragen (Abbildung 4.32). Wie oben beschrieben, zeichnet sich ein Trend für die Polyamine C3N6, C3N12 und C3N18 ab, der sich mit C3N12Me fortsetzt. In dieser nimmt die Hydrophobizität $\mathrm{zu}$, die Ladung nimmt $\mathrm{ab}$ und gleichzeitig steigt das Präzipitationspotenzial. In der Reihe C2N7 bis C4N7 steigt die Siliciummasse mit der Protonierung bei fast gleichbleibender Hydrophobizität.

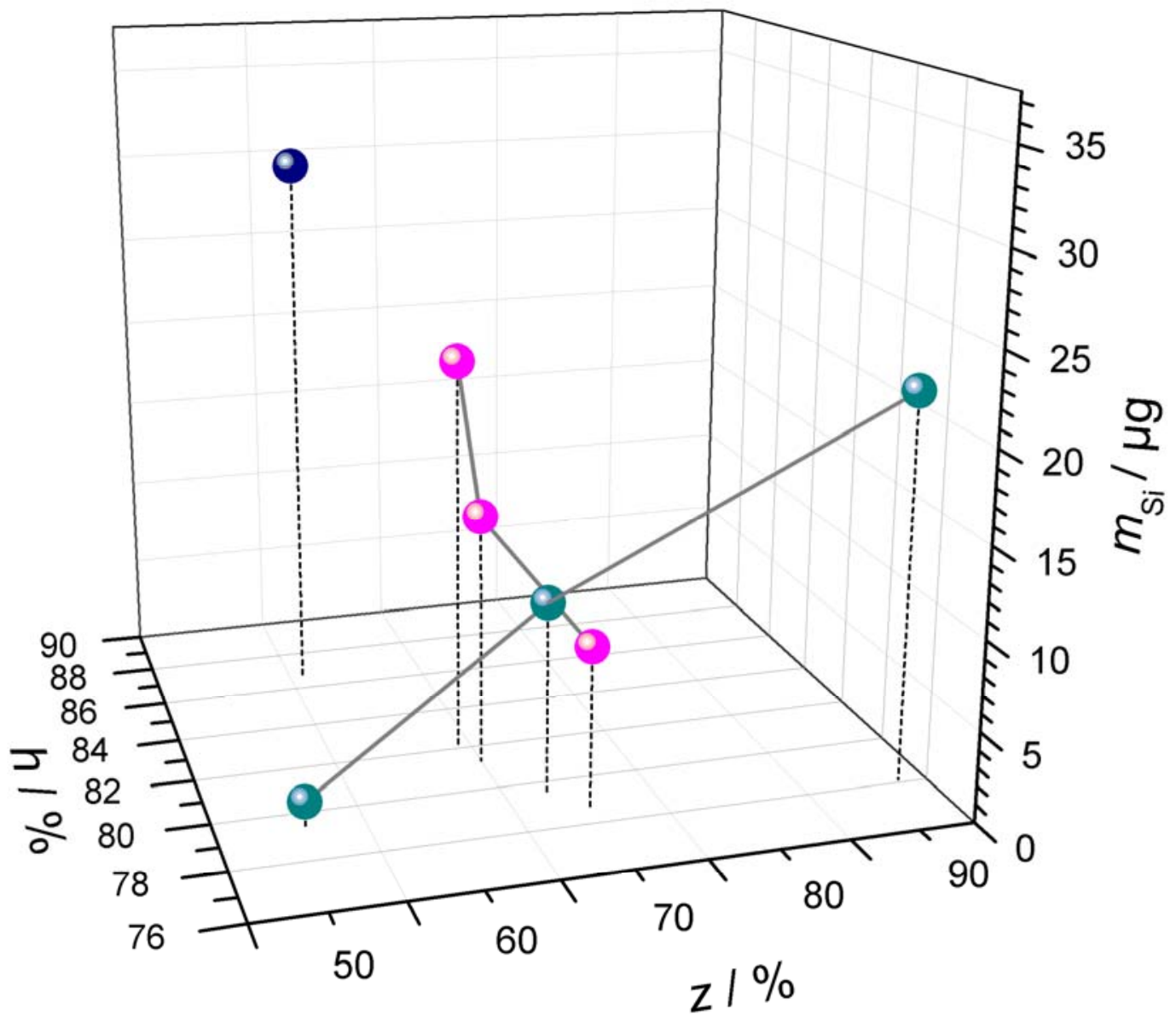

Abbildung 4.32 Zusammenhang zwischen präzipitierter Siliciummasse $m_{\mathrm{Si}}$, Protonierungsgrad $z$ und hydrophobem Charakter $h$ bei pH 6,8 der Polyamine $\bullet$ C3N6, C3N12, C3N18 (von links nach rechts); - C2N7, C3N7, C4N7 (von vorne nach hinten) und $\bullet$ C3N12Me. 
Aufgrund der zuvor erläuterten Aspekte können mehrere Hypothesen bezüglich der Phasenseparation von Polyaminen in wässriger Lösung und sich anschließender Silicapräzipitationen aufgestellt werden:

- Je hydrophober und gleichzeitig weniger stark geladen ein Polyamin ist, desto stärker ist die Vernetzung der Moleküle und desto deutlicher ausgeprägt die Phasenseparation.

- Bei ähnlicher Hydrophobizität bewirkt eine Erhöhung der Protonierung eine stärkere Vernetzung und Aggregation.

- Je ausgeprägter die Aggregation der Polyamine ist, desto mehr Silica wird gebildet und desto größer sind durchschnittlich die gebildeten Silicapartikel.

- Die Erhöhung der Konzentration von multivalenten Anionen resultiert in einer Erhöhung der gebildeten Silicamenge.

- Die Mindestkonzentration an Anionen, die nötig ist, jeweils maximale Präzipitation zu induzieren, ist umso geringer, je größer die Polyaminkettenlänge ist.

- Bei großen Polyaminkettenlängen und hohen Polyaminkonzentrationen aggregieren Polyamine in Anwesenheit eines Überschusses an multivalenten Anionen nicht ausschließlich zu Tröpfchen, sondern auch zu faserförmigen Strukturen. Dadurch wird die Bildung von filamentartigem Silica bedingt.

- Die Silicapolymerisationsreaktion findet an der Grenzfläche zwischen Polyaminaggregat und wässriger Umgebung statt, wodurch ausschließlich hohle Kugeln gebildet werden.

Diese Zusammenhänge sind grafisch in Abbildung 4.33 zusammengefasst. Es war zwar im Rahmen dieser Arbeit nicht möglich, den direkten Einfluss der Polyamine auf den Reaktionsmechanismus zu klären. Auch kann keine Aussage darüber getroffen werden, auf welchen Ebenen die Polyamine genau die Silicabildung fördern. Es ist jedoch wahrscheinlich, dass nicht ein einzelner Aspekt wie die Beeinflussung der Kondensationsreaktion, die Verbrückung von Silicapartikeln oder die Phasenseparation der Polyamine allein entscheidend ist, sondern vielmehr ein Zusammenspiel dieser Faktoren. In ihrer Gesamtheit korrespondieren die Erkenntnisse mit dem von Sumper ${ }^{32}$ vorgeschlagenen Phasenseparationsmodell, das die Vorgänge auf der Nanoskala der Silicabildung zu erklären versucht. Auch andere Modelle und Vorstellungen wie etwa die von Patwardhan et al. ${ }^{132}$ beziehungsweise Vrieling et al. ${ }^{33}$ stehen im Einklang mit den vorgestellten Ergebnissen. 

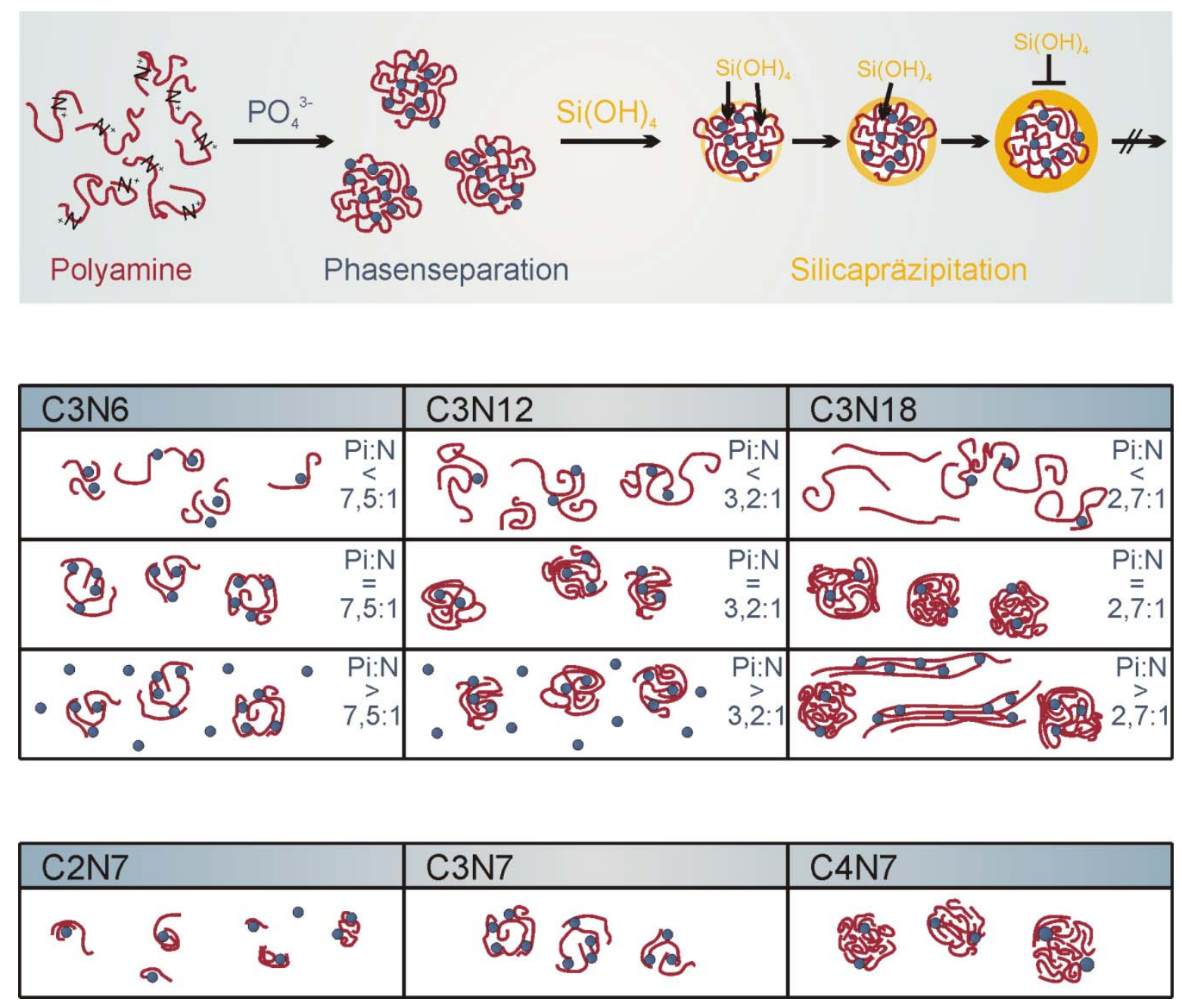

Abbildung 4.33 Grafische Darstellung der Modellvorstellungen bezüglich Silicapräzipitation und phosphatinduzierter Polyaminaggregation. Oben: Die positiv geladenen Polyamine werden über Phosphationen miteinander vernetzt, wodurch es zu Phasenseparation kommt. Nach Zugabe von Kieselsäure wird die Polymerisation an der Grenzfläche zwischen Polyaminaggregat und wässriger Lösung induziert. Erreicht die Silicaschale eine gewisse Schichtdicke, wird die Diffusion von Kieselsäure in Richtung der Polyamine unterbunden, die Reaktion kommt zum Erliegen. Hierbei entstehen hohle Silicakugeln. Mitte: Mit zunehmender Kettenlänge (C3N6 bis C3N12) und damit steigender Hydrophobizität sowie analog leicht abnehmender Protonierung wird die Vernetzung zwischen den Polyaminen verstärkt. Aus diesem Grund nimmt in dieser Reihe die Mindestkonzentration an Phosphationen ab, die benötigt wird, um maximale Präzipitation zu erhalten. Weiterhin nehmen die Aggregatgrößen von C3N6 bis C3N18 zu. Im Fall von C3N18 kommt es in Anwesenheit überschüssiger Phosphationen zur Bildung filamentartiger Polyaminaggregate. Unten: Die Verlängerung der Alkylkette von C2N7 bis zu C4N7 hat eine deutliche Zunahme der Ladung bei gleichbleibender Hydrophobizität zur Folge, was zu einer verstärkten Aggregation und einer Zunahme der Aggregatgröße von Polyaminen führt. 


\section{Aminolipide in Vesikeln}

Die in Kapitel 4 beschriebenen polyamininduzierten Silicabildungsexperimente können zur Klärung der in Diatomeen ablaufenden Vorgänge beitragen. Die Bildung definierter $\mathrm{SiO}_{2}-$ Strukturen unterliegt in diesem System jedoch einigen Einschränkungen. Durch Modifikation der Polyaminstrukturen und Veränderung der Reaktionsbedingungen kann die Morphologie des Silica zwar bis zu einem gewissen Grad beeinflusst werden. Letztendlich bleibt die Silicastruktur jedoch von der Aggregation der Polyamine abhängig und diese kann nicht beliebig verändert werden. Aus diesem Grund wurden verschiedene biomimetische Ansätze verfolgt, bei denen versucht wurde, die Strukturen zu beeinflussen, indem beispielsweise Polyamine elektrostatisch an Oberflächen koordiniert ${ }^{133,134}$ oder kovalent an Strukturen-bildende Moleküle gebunden wurden. ${ }^{135}$ Es wurden auch weitere Silica präzipitierende Moleküle verwendet, die definierte Template in Lösungen ausbilden. $^{136}$

In der vorliegenden Arbeit wurde ein biomimetischer Ansatz verfolgt, in dem die Verwendung amphiphiler lipidähnlicher Moleküle die Ausbildung von Templaten für Silicapräzipitationen ermöglichen sollte. Diese Moleküle enthalten, wie Silaffine beziehungsweise Polyamine, Aminogruppen, die die Bildung von Silica induzieren sollen, woraus sich die mitunter verwendete Bezeichnung Silaffin-Analoga ableitet. Sie besitzen, ähnlich wie Lipide, hydrophobe Alkylketten und eine hydrophile Kopfgruppe, in der jeweils eine oder mehrere Aminogruppen beinhaltet sind (Abbildung 3.2) und werden deshalb an dieser Stelle auch Aminolipide genannt.

Über Lipide ist bekannt, dass sie in lamellaren Systemen aggregieren, in denen die Moleküle in Doppelschichten angeordnet sind. Etablierte Präparationsmethoden erlauben den Aufbau von Vesikeln verschiedener Durchmesser beziehungsweise den von festkörperunterstützten Membranen. Auch hierbei spielen, wie bei den Polyaminen, selbstorganisierende Prozesse eine wichtige Rolle, doch hängen diese von anderen Faktoren ab und können stärker beeinflusst werden. Die Anordnung der Moleküle in großen Überstrukturen ist von ihrer Geometrie, der Präparationsmethode und Reaktionsbedingungen, wie pH-Wert und Ionenstärke, abhängig.

Zunächst galt es zu untersuchen, ob mit den Aminolipiden lamellare Aggregate, genauer Vesikel, gebildet werden können. Bei Erfüllung dieser Voraussetzung, sollten anschließend die Silica-präzipitierenden Eigenschaften solcher Vesikel getestet werden. Dabei war der Einfluss des $N$-Methylierungsmusters auf die Silicabildung von besonderem Interesse. Die 
Aminolipide eignen sich hierfür als Modellsubstanzen, da ihr Aufbau nahezu identisch ist und sie sich lediglich in der $N$-Methylierung beziehungsweise der Anzahl der Aminogruppen unterscheiden (Tabelle 5.1). Gelingt es, mit allen Sillafin-Analoga ähnliche Aggregate zu bilden, bietet sich dadurch die Möglichkeit, die Silicapräzipitation reduziert auf die Abhängigkeit von den Variationen in den Aminogruppen zu untersuchen. Hieraus könnten weitere Einsichten zu den bei Präzipitationsreaktionen ablaufenden Vorgängen und Mechanismen gewonnen werden.

\subsection{Strukturen und Eigenschaften der Aminolipide}

Der Aufbau des primären (pAL), sekundären (sAL), tertiären (tAL) und quartären Aminolipids (qAL) ist nahezu identisch. Alle vier Substanzen beinhalten zwei Dodecylketten, die über Etherbindungen mit einem Glycerinmolekül verknüpft sind. Die dritte Hydroxygruppe des Glycerin ist durch eine Aminogruppe ausgetauscht, welche die Kopfgruppe des Moleküls bildet. Der Unterschied zwischen den Verbindungen liegt in ihrem $N$-Methylierungsmuster. In Tabelle 5.1 sind die entsprechenden Struktur- und Summenformeln aufgeführt.

Tabelle 5.1 Eigenschaften des primären (pAL), sekundären (sAL), tertiären (tAL), quartären (qAL) Aminolipids sowie des Lysinollipid (LysinolL) bei pH 6,0.

\begin{tabular}{|c|c|c|c|c|c|}
\hline & Abkürzung & $N_{N}$ & $M I(\mathrm{~g} / \mathrm{mol})$ & Strukturformel & Summenformel \\
\hline A & $\mathrm{pAL}$ & 1 & 427,8 & & $\mathrm{C}_{27} \mathrm{H}_{57} \mathrm{NO}_{2}$ \\
\hline B & sAL & 1 & 441,8 & & $\mathrm{C}_{28} \mathrm{H}_{59} \mathrm{NO}_{2}$ \\
\hline C & $\mathrm{tAL}$ & 1 & 455,8 & & $\mathrm{C}_{29} \mathrm{H}_{61} \mathrm{NO}_{2}$ \\
\hline D & $q A L$ & 1 & 470,8 & & $\mathrm{C}_{30} \mathrm{H}_{64} \mathrm{NO}_{2}^{+}$ \\
\hline E & LysinolL & 2 & 542,9 & & $\mathrm{C}_{33} \mathrm{H}_{72} \mathrm{~N}_{2} \mathrm{O}_{3}$ \\
\hline
\end{tabular}

Hier finden sich weiterhin Angaben zur Anzahl der Aminoheiten pro Molekül $N_{\mathrm{N}}$ und der molaren Masse $M$ ohne Berücksichtigung von Gegenionen. Verbindung E (Lysinollipid) besitzt eine andere Struktur als die Aminolipide A-D. Wiederum sind zwei Dodecylketten 
über Etherbindungen mit einem Glycerinmolekül verknüpft. An die dritte Hydroxygruppe von Glycerin ist über eine Etherstruktur die Kopfgruppe gebunden, bei der es sich um Lysinol handelt, dem Alkoholderivat der Aminosäure Lysin. Lysinol weist zwei primäre Aminoeinheiten auf.

\subsection{Bestimmung der Aggregatgrößen}

Um das Präzipitationspotenzial in Abhängigkeit der verschiedenen Aminoeinheiten untersuchen zu können, galt es zunächst, aus allen Aminolipiden Aggregate ähnlicher Größe zu bilden. Die Bildung mizellarer oder vesikulärer Strukturen aus amphiphilen Substanzen unterliegt unter anderem der Geometrie der Moleküle. ${ }^{137}$ Diese wird über den Packungsparameter $P$ ausgedrückt:

$$
P=V / a_{0} l_{c}
$$

Hierbei steht $V$ für das effektive Volumen der hydrophoben Ketten des Moleküls, $l_{\mathrm{c}}$ für die kritische Länge der unpolaren Ketten und $a_{0}$ bezeichnet die optimale Fläche der polaren Kopfgruppe, bei welcher das chemische Potential $\mu_{0}$ minimal ist. ${ }^{138}$ Für $0,5 \leq P \leq 1$ lagern sich Lipide zu lamellaren Strukturen zusammen, also zu Doppelschichten in Form von Vesikeln oder planaren Membranen. Im Fall von $P \leq 0,5$ ist hingegen die Bildung von mizellaren Strukturen bevorzugt. ${ }^{139}$ Je größer die effektive Kopfgruppengröße relativ zum hydrophoben Teil ist, desto kleiner ist der Wert von $P$. Bei einem amphiphilen Molekül hängt die effektive Kopfgruppengröße nicht allein vom Van-der-Waals-Volumen ab, sondern wird zudem durch laterale Kräfte bestimmt. ${ }^{140}$ Diese werden durch Parameter wie Dipol- und elektrische Felder, Wechselwirkungen mit benachbarten Gruppen durch beispielsweise Wasserstoffbrücken oder Hydratation der Kopfgruppen beeinflusst. ${ }^{137,141}$ Äußere Bedingungen wie pH-Wert und Ionenstärke spielen hierbei eine Rolle. Aus diesem Grund wurden Puffer mit verschiedenen Ionenstärken verwendet. Um einen Einblick zu erhalten, in welcher Aggregatform die Aminolipide in diesen Puffern vorliegen, wurden mittels dynamischer Lichtstreuung (DLS) die hydrodynamischen Radien der Aggregate in Lösung bestimmt.

Von allen fünf Substanzen wurde jeweils eine Suspension hergestellt ( $c=1 \mathrm{mM}$ ), indem Filme der Substanzen mit Puffer rehydratisiert und im Anschluss mehrmals geschüttelt wurden (vgl. Kapitel 3.2.1). Dabei entstanden klare oder opaleszente Suspensionen. Diese 
wurden durch Polycarbonatmembranen mit Porendurchmessern von $200 \mathrm{~nm}$ extrudiert. Die entstandenen Aggregate wurden sofort nach der Präparation bezüglich ihrer Größen und deren Verteilung mittels DLS untersucht.
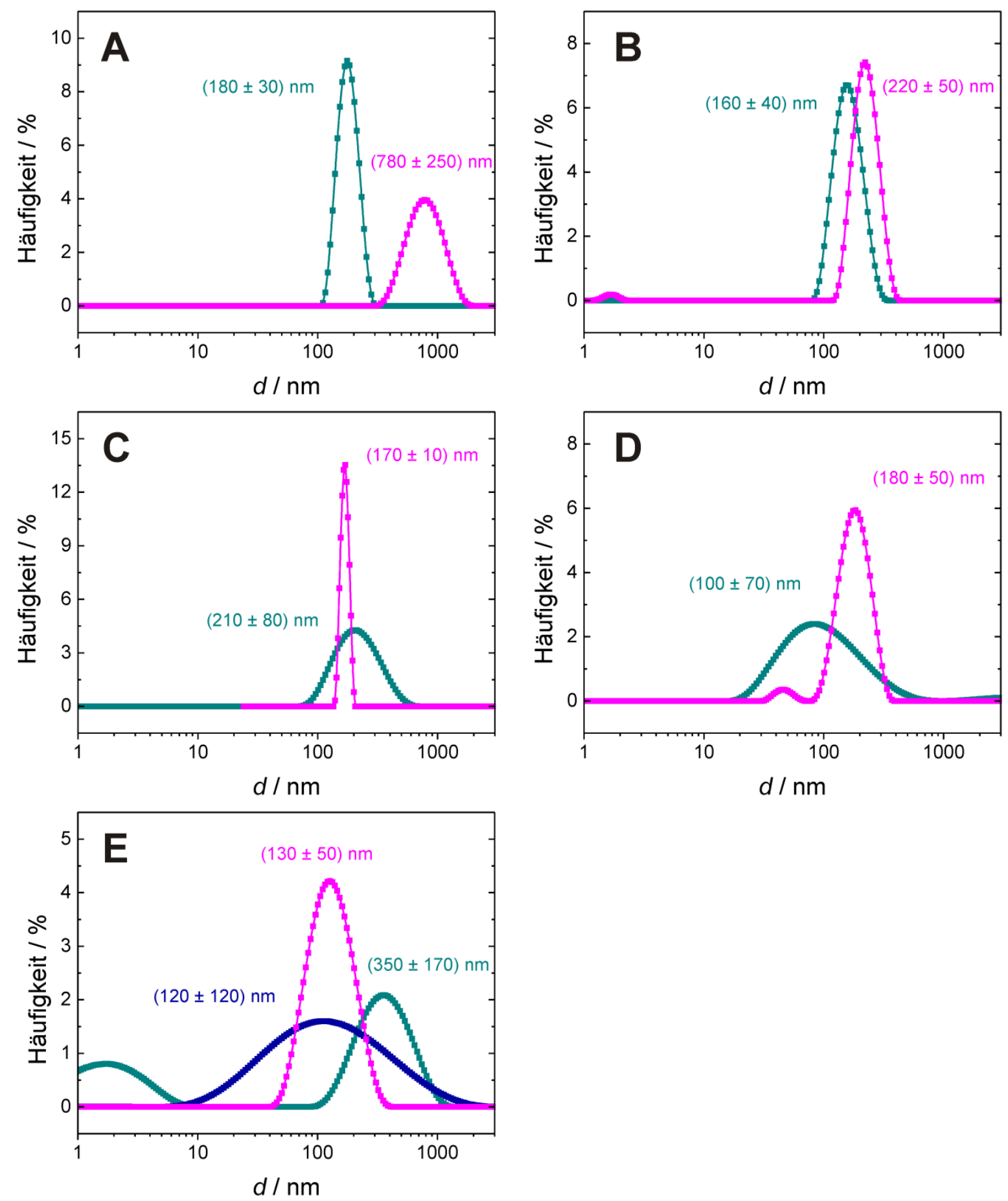

Abbildung 5.1 Mittels dynamischer Lichtstreuung bestimmte Verteilungen der Durchmesser von Vesikeln der Aminolipide A pAL, B sAL, C tAL, D qAL und $\mathbf{E}$ Lysinollipid in $50 \mathrm{mM}$ MES, - $25 \mathrm{mM} \mathrm{MES} / 500 \mathrm{mM} \mathrm{KCl}$ oder $-40 \mathrm{mM} \mathrm{MES} / 200 \mathrm{mM} \mathrm{KCl}$. Bedingungen: $c_{\text {Lipid }}=1 \mathrm{mM}, \mathrm{pH}$ 6,0.

Die Ergebnisse (Abbildung 5.1) zeigen, dass alle Verbindungen Aggregate bilden, die eine Größe von mehr als $50 \mathrm{~nm}$ besitzen. Es kann davon ausgegangen werden, dass Partikel mit 
Durchmessern über $50 \mathrm{~nm}$ Vesikel darstellen, während solche im Größenbereich unter $10 \mathrm{~nm}$ mizellare Struktur besitzen. ${ }^{142,143}$ Da die Suspensionen durch Polycarbonatmembranen mit Porendurchmessern von $200 \mathrm{~nm}$ extrudiert wurden, wird bei der Bildung von Vesikeln eine Verteilung in der Größenordnung von $d=200 \mathrm{~nm}$ erwartet. Vesikelsuspensionen des primären Aminolipids wurden bei einer erhöhten Temperatur von $60{ }^{\circ} \mathrm{C}$ hergestellt, da sich bei Raumtemperatur keine homogenen Suspensionen bildeten. In MES-Puffer ergab sich hierbei ein Vesikeldurchmesser von $(180 \pm 30) \mathrm{nm}$ (Abbildung 5.1 A). Wird MES/KCl-Puffer verwendet, so ist der mittlere Durchmesser größer und beträgt (780 \pm 250$) \mathrm{nm}$. Die Präparation der Aggregate der vier anderen Verbindungen wurde bei Raumtemperatur durchgeführt. Bei Verwendung des sekundären Aminolipids (Abbildung 5.1 B) in MES-Puffer entstanden Vesikel einer Größe von (160 \pm 40$) \mathrm{nm}$. Mit $(220 \pm 50) \mathrm{nm}$ ist die Verteilung bei höherer Ionenstärke in MES/KCl-Puffer sehr ähnlich. Ähnliche Verhältnisse zeigen sich beim tertiären Aminolipid (Abbildung 5.1 C). Während in MES-Puffer eine relativ breites Signal bei $(210 \pm 80) \mathrm{nm}$ auftritt, wurde im Puffersystem $\mathrm{MES} / \mathrm{KCl}$ ein sehr scharfer Peak bei $(170 \pm 10) \mathrm{nm}$ detektiert. Mit dem quartären Aminolipid (Abbildung 5.1 D) zeichnet sich in MES-Puffer mit (100 \pm 70$) \mathrm{nm}$ ein etwas kleinerer Vesikeldurchmesser als in MES/KCl-Puffer ab, wo er $(180 \pm 50) \mathrm{nm}$ beträgt.

Mit dem Lysinollipid wurden drei verschiedene Präparationsbedingungen untersucht (Abbildung 5.1 E). Zunächst wurde, wie mit den Aminolipiden, ein MES-Puffer niedriger Ionenstärke und ein MES/KCl-Puffer hoher Ionenstärke verwendet. Unter Verwendung von MES-Puffer ohne Kaliumchlorid ist kein einzelner Peak erkennbar, vielmehr gibt es zwei Signale. Bei diesem System variierten die Werte von Messung zu Messung stärker, als bei den anderen, was darauf schließen lässt, dass die Verteilung hier insgesamt heterogener war. In allen Fällen trat jedoch ein Signal im Bereich von $d<10 \mathrm{~nm}$ auf und ein weiteres im Bereich von $(180-800) \mathrm{nm}$. Abbildung 5.1 E gibt das Ergebnis einer repräsentativen Messung wieder. Hier lag der Durchmesser der anwesenden Vesikel bei $(350 \pm 170) \mathrm{nm}$. Der zugehörige Peak weist jedoch eine geringe Intensität auf und es ist zusätzlich ein starkes Signal unter $10 \mathrm{~nm}$ erkennbar, das Mizellen zugewiesen werden kann. Wird ein Puffer, bestehend aus $25 \mathrm{mM} \mathrm{MES} / 500 \mathrm{mM} \mathrm{KCl}$, verwendet und nicht weiter verdünnt, tritt eine einzelne Verteilung auf, die Vesikel haben dann einen Durchmesser von $(130 \pm 50) \mathrm{nm}$.

Da in MES, wie oben erläutert, mizellare und vesikuläre Aggregate detektiert wurden, in $\mathrm{MES} / \mathrm{KCl}$ jedoch die Präzipitation wenig stark ausgeprägt war, worauf im nächsten Kapitel näher eingegangen wird, wurde mit dem Lysinolllipid noch eine dritte Präparationsvariante 
untersucht. Dabei wurde die Suspension zunächst bei hoher Ionenstärke $(\mathrm{MES} / \mathrm{KCl})$ hergestellt und extrudiert, um eine gute Vesikelbildung zu gewährleisten. Im Anschluss daran wurde die Suspension mit MES-Puffer verdünnt, wodurch die Ionenstärke wieder verringert wurde, was für später durchzuführende Präzipitationsreaktionen von Vorteil sein könnte. Die letztendlichen Konzentrationen betrugen $c_{\mathrm{MES}}=40 \mathrm{mM}$ und $c_{\mathrm{KCl}}=200 \mathrm{mM}$. In diesem Fall waren die Signale, die von Mizellen stammen, deutlich weniger intensiv, als dies in reinem MES-Puffer der Fall war. Machten im MES-Puffer die Signale im Bereich unter $5 \mathrm{~nm}$ noch einen Anteil von ca. $80 \%$ aus, traten hier teilweise keine unter $10 \mathrm{~nm}$ auf, woraus geschlossen werden kann, dass der Anteil der Aggregate zu Vesikeln hin verschoben wurde. Wiederum traten Variationen innerhalb der Messreihe auf, es gab teilweise ein Signal bei $d<5 \mathrm{~nm}$, sowie immer auftretend einen Peak zwischen ca. $50 \mathrm{~nm}$ und $450 \mathrm{~nm}$. Die abgebildeten Daten, bei denen der Vesikeldurchmesser um $(120 \pm 120) \mathrm{nm}$ liegt, geben ein repräsentatives Ergebnis wieder.

Die Stabilität der Aggregate wurde zwar nicht systematisch untersucht, doch einige Suspensionen wurden nicht direkt nach Extrusion, sondern erst einige Stunden später vermessen. In diesen Fällen traten teilweise deutlich größere Partikel auf als direkt nach der Präparation. So wiesen beispielsweise Vesikel von qAL in MES/KCl $10 \mathrm{~h}$ nach ihrer Herstellung Durchmesser von $720 \mathrm{~nm}$ auf, die von pAL in MES nach ca. $9 \mathrm{~h} 400 \mathrm{~nm}$, solche von sAL in MES/KCl nach 6,5 h ca.1000 nm und die von tAL in MES 5,5 h nach der Präparation ca. 320 nm. Auf Aggregations- oder Fusionsprozesse deutet hin, dass die gebildeten Aggregate bezüglich ihrer Größe nicht stabil sind. Dies erklärt den großen Durchmesser der Vesikel aus primärem Aminolipid von $780 \mathrm{~nm}$. Die Fusion zu größeren Vesikeln wurde in diesem Fall vermutlich durch die erhöhte Temperatur bei der Präparation beschleunigt.

Zusammenfassend lässt sich feststellen, dass das primäre, sekundäre, tertiäre und quartäre Aminolipid in beiden Puffersystemen ausschließlich in vesikulären Strukturen vorliegen, deren Größen im erwarteten Bereich von $d \approx 200 \mathrm{~nm}$ liegen. Beim Lysinollipid bewirkte eine Erhöhung der Ionenstärke eine deutliche Verschiebung von mizellaren hin zu vesikulären Strukturen. Da alle Aminolipide Vesikel bilden und deren Durchmesser größtenteils im gleichen Bereich liegen, sind die Voraussetzungen die nachfolgend vorgestellten Silicapräzipitationsexperimente gegeben. 


\subsection{Silicapräzipitationsexperimente}

Nachdem durch die dynamischen Lichtstreuungsexperimente sichergestellt werden konnte, dass die Aminolipide Aggregate in Form von Vesikeln bilden, wurden ihre Präzipitationsinduzierenden Eigenschaften untersucht. Da die Konzentration der Aminolipide in allen Lösungen identisch war und die Aggregate ähnlich groß sind, kann auch davon ausgegangen werden, dass die jeweilige Anzahl der Vesikel in den Lösungen nicht stark variiert und dadurch ähnlich große Oberflächen zur Verfügung stehen, an denen die Reaktionen ablaufen können. Die Lösungen wurden mit Monokieselsäure versetzt und nach zehn Minuten zentrifugiert, um die Reaktion zu stoppen. Der Niederschlag wurde mit Wasser gewaschen, bevor das entstandene Silica mit Natronlauge hydrolysiert wurde. Im Anschluss wurden die Si-Massen in allen Proben mittels der $\beta$-Molybdatmethode (siehe Kapitel 3.3.1) bestimmt. Einen Überblick über die Ergebnisse gibt Abbildung 5.2.

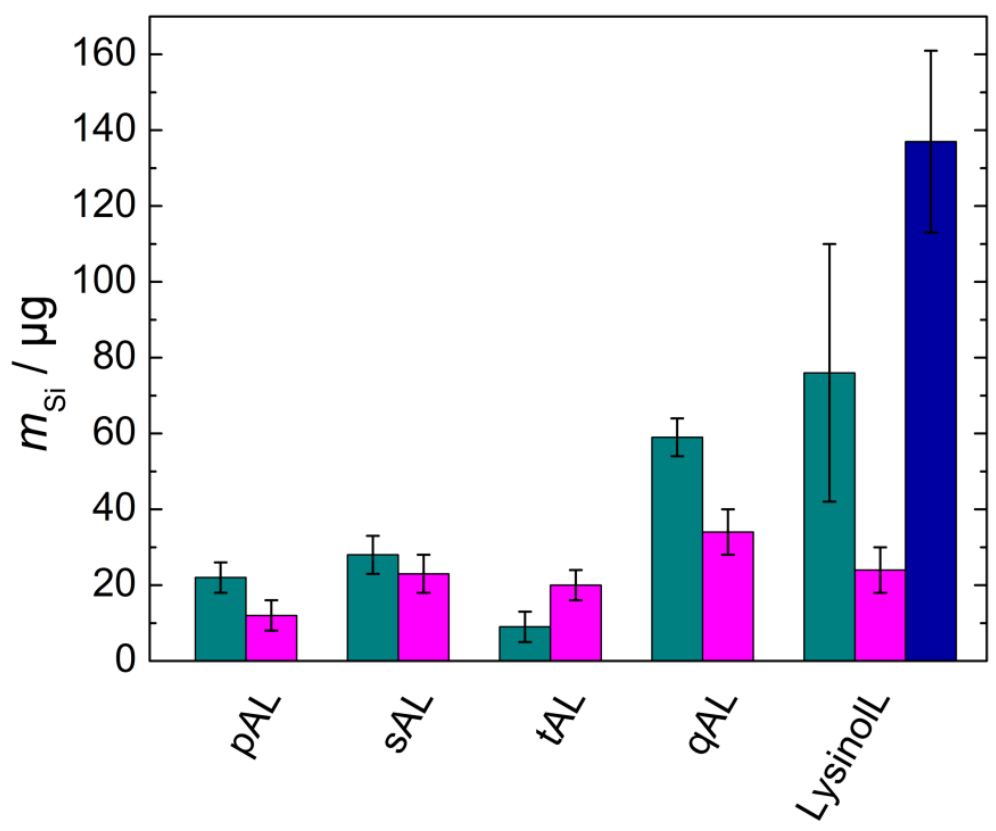

Abbildung 5.2 Überblick über die mittels $\beta$-Molybdatmethode bestimmten Massen $m_{\mathrm{Si}}$ an präzipitiertem $\mathrm{SiO}_{2}$ für die Aminolipide pAL, sAL, tAL, qAL und Lysinollipid in $50 \mathrm{~mm} \mathrm{MES}$, - $25 \mathrm{mM}$ MES/500 mM KCl oder - 40 mM MES/200 mM KCl. Reaktionsbedingungen: pH 6,0, $c_{\text {Lipid }}: 1$ mM, $c_{(\mathrm{SiOH})_{4}}=100 \mathrm{mM}$, Reaktionszeit: $10 \mathrm{~min}$. 
Der Vergleich der präzipitierten Silicamengen in $50 \mathrm{mM}$ MES-Puffer, gemittelt über fünf Messungen $(n=5)$, liefert folgende Reihenfolge:

tAL $(9 \pm 4) \mu \mathrm{g}<\mathbf{p A L}(22 \pm 4) \mu \mathrm{g}<\mathbf{s A l}(28 \pm 5) \mu \mathrm{g}<\mathbf{q A L}(59 \pm 5) \mu \mathrm{g}<\mathbf{L y s i n o l L}(76 \pm 34) \mu \mathrm{g}$

Auch in $25 \mathrm{mM} \mathrm{MES/500} \mathrm{mM} \mathrm{KCl} \mathrm{unterscheiden} \mathrm{sich} \mathrm{die} \mathrm{Silicamengen,} \mathrm{hier} \mathrm{ergab} \mathrm{sich}$ nach jeweils sechs Messungen $(n=6)$ eine Reihenfolge von:

pAL $(12 \pm 4) \mu \mathrm{g}<\mathbf{t A L}(20 \pm 4) \mu \mathrm{g}<\mathbf{s A L}(23 \pm 5) \mu \mathrm{g}<$ LysinolL $(24 \pm 6) \mu \mathrm{g}<\mathbf{q A L}(34 \pm 6) \mu \mathrm{g}$

Die mit $(137 \pm 24) \mu \mathrm{g}$ weitaus größte Masse an Silicium wurde bei der Präzipitation beobachtet, die durch das Lysinollipid im Puffersystem $40 \mathrm{mM} \mathrm{MES} / 200 \mathrm{mM} \mathrm{KCl}$ ausgelöst wurde, welche fünfmal durchgeführt wurde $(n=5)$.

Zusammenfassend betrachtet induzieren alle fünf Aminolipide bei pH 6,0 innerhalb von zehn Minuten die Bildung von Silica aus Monokieselsäure. Hervorzuheben ist, dass hierbei, anders als mit Polyaminen (Kapitel 4), die Präzipitation in Abwesenheit von multivalenten Anionen stattfindet. Obwohl ungefähr gleich viele ähnlich große Aggregate in den Lösungen vorhanden sind, zeigen sich quantitative Unterschiede bei der $\mathrm{SiO}_{2}$ Bildung als Funktion des Aminolipids und damit des $N$-Methylierungsmusters. Weiterhin wird bei niedriger Ionenstärke in Abwesenheit von Kaliumchlorid durch alle Aminolipide, außer dem tertiären, mehr Silica gebildet.

Um weitere Informationen zu den Präzipitationsreaktion an Vesikeln der Aminolipide, zusätzlich zur Menge an Präzipitat, zu gewinnen, wurden diese auch mittels dynamischer Lichtstreuung verfolgt. Hierbei ist eine Beobachtung des zeitlich Verlaufs möglich, die die zuvor beschriebenen Messungen nicht erlaubten. Nach Bestimmung der Aggregatgrößen (Abbildung 5.1) wurde $\mathrm{zu}$ den Vesikelsuspensionen direkt in der Lichtstreuungsmesskammer Monokieselsäure in einer Konzentration von $100 \mathrm{mM}$ zugegeben und anschließend der zeitliche Verlauf der Änderung des hydrodynamischen Radius sowie der Intensität des Signals verfolgt. Bei allen Aminolipiden setzte innerhalb von 1 - 4 Minuten nach Zugabe der Kieselsäure ein Anstieg der Streuintensität ein, die zuvor konstant war. Zugleich stiegen die gemessenen hydrodynamischen Radien innerhalb weniger Minuten deutlich an. Exemplarisch ist eine Messung mit dem Lysinollipid im Puffersystem $40 \mathrm{mM} \mathrm{MES} / 200 \mathrm{mM} \mathrm{KCl}$ in Abbildung $5.3 \mathrm{~A}$ dargestellt. Die Zugabe von $\mathrm{Si}(\mathrm{OH})_{4}$ erfolgte zum Zeitpunkt $t=0 \mathrm{~min}$. Zu jedem gemessenen Zeitpunkt wurde die Verteilung der Radien innerhalb der Lösung detektiert. In der Auftragung ist die Änderung des Mittelwerts desjenigen Peaks dargestellt, der zu Anfang bei einem Durchmesser von 
$110 \mathrm{~nm}$ lag. Innerhalb der ersten 3,5 Minuten verändert sich der hydrodynamische Radius der Lysinollipidvesikel nicht. Dann setzt ein rascher Anstieg ein, bereits nach 7,5 Minuten beträgt $r_{\mathrm{H}}$ ca. $3800 \mathrm{~nm}$. Ab diesem Zeitpunkt gibt es keine wesentliche Änderung mehr, der Wert schwankt um ca. $4050 \mathrm{~nm}$. Interessant ist, dass im Verlauf der Messung des dargestellten Systems nur noch ein Peak auftrat, obwohl vor der Kieselsäurezugabe mehrere vorlagen (vergleiche Abbildung 5.1 E).
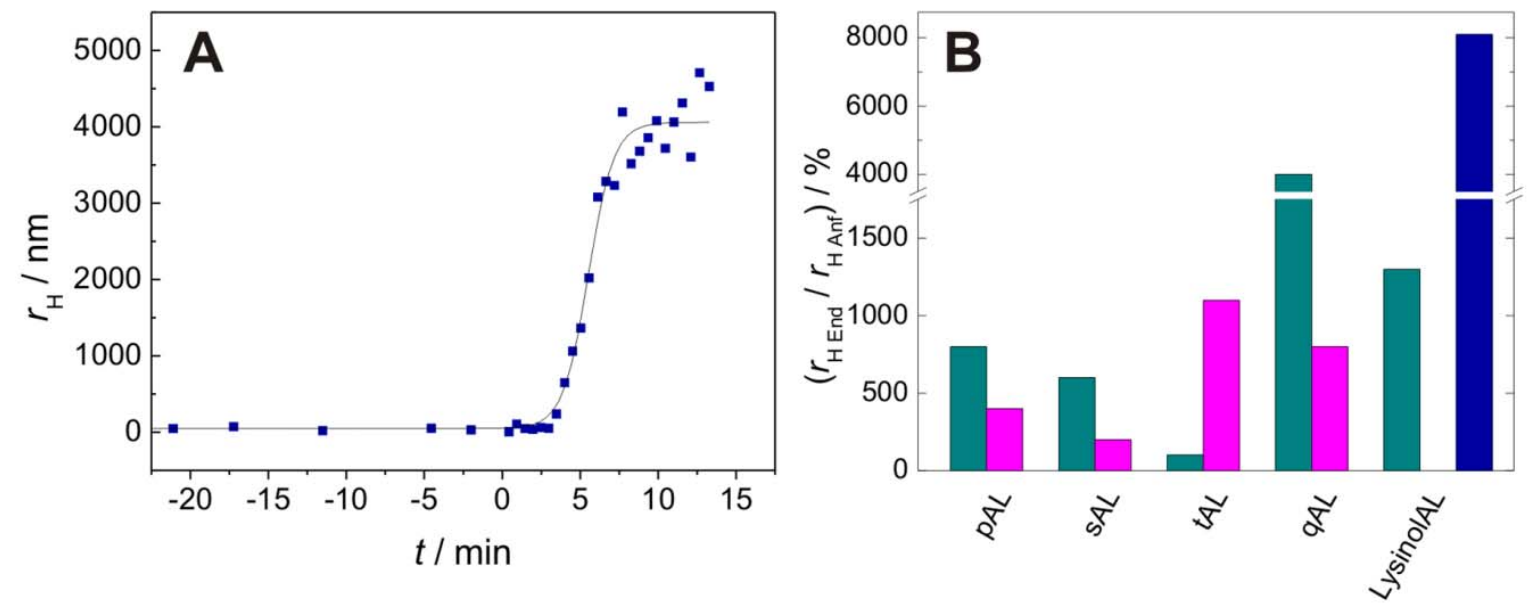

Abbildung 5.3 Dynamische Lichtstreuungsmessungen: A Zeitliche Änderung des hydrodynamischen Radius einer Lösung von Vesikeln des Lysinollipids in $40 \mathrm{mM} \mathrm{MES} / 200 \mathrm{mM} \mathrm{KCl}$ nach Zugabe von Si(OH) $)_{4} \mathrm{zum}$ Zeitpunkt $t=0 \mathrm{~min}$. Die schwarze Linie zeigt eine Anpassung an die Daten, die zur Ermittlung von $r_{\mathrm{H}} \mathrm{zu}$ Beginn und Ende der Messung und somit zur Bestimmung von $\Delta r_{\mathrm{H}}$ durchgeführt wurde. B Prozentuale Änderung des hydrodynamischen Radius in Abhängigkeit der verwendeten Aminolipide und der Puffer - $50 \mathrm{~mm} \mathrm{MES}$, $25 \mathrm{mM} \mathrm{MES} / 500 \mathrm{mM} \mathrm{KCl}$ oder $40 \mathrm{mM} \mathrm{MES} / 200 \mathrm{mM} \mathrm{KCl}$. Reaktionsbedingungen: pH 6,0, $c_{\text {Lipid }}: 1 \mathrm{mM}, c_{(\mathrm{SiOH})_{4}}=100 \mathrm{mM}$, Reaktionszeit: $12-15 \mathrm{~min}$.

Für alle Aminolipide ergab sich in den eingesetzten Puffersystemen ein ähnlicher Zeitverlauf. Unterschiedlich waren jedoch die relativen Änderung der hydrodynamischen Radien der fünf Verbindungen (Abbildung 5.3 B). Die relativen Änderungen entsprechen dem Quotienten aus hydrodynamischem Radius 12-15 Minuten nach Zugabe von $\mathrm{Si}(\mathrm{OH})_{4}$ $\left(r_{\mathrm{H} \text { End }}\right)$ und demjenigen vor der Zugabe $\left(r_{\mathrm{H} \text { Anf }}\right)$. Im Überblick ergaben sich in $50 \mathrm{mM}$ MES pH 6,0 folgende Zunahmen des hydrodynamischen Radius:

tAL $(100 \%)<$ sAL $(600 \%)<$ pAl $(800 \%)<$ LysinolL $(1300 \%)<$ qAL $(4000 \%)$

In $25 \mathrm{mM} \mathrm{MES/500} \mathrm{mM} \mathrm{KCl} \mathrm{pH} \mathrm{6,0} \mathrm{stellte} \mathrm{sich} \mathrm{die} \mathrm{Reihenfolge} \mathrm{folgendermaßen} \mathrm{dar:}$

LysinolL (0 \%) < sAL (200\%) < pAL (400\%) < qAl (800\%) < tAL (1100\%)

Eine Zunahme von $8100 \%$ im Fall des Lysinollipids in $40 \mathrm{~mm} \mathrm{MES/200} \mathrm{mM} \mathrm{KCl}$ bedeutete die stärkste Änderung aller durchgeführten Messungen. 
Die hierbei auftretenden Trends ähneln denen, die bei der Bestimmung der präzipitierten Si-Massen mit dem $\beta$-Molybdattest beobachtet wurden (Abbildung 5.2). Sowohl bei pAl, sAL, qAL als auch beim Lysinollipid war der beobachtete Effekt in MES-Puffer stärker ausgeprägt als in Anwesenheit von KCl. Auch bei tAL war der Trend gleich, hier trat bei höherer Ionenstärke eine größere Zunahme von $r_{\mathrm{H}}$ auf.

Zur Überprüfung der Aussagekraft der Ergebnisse wurden zwei Kontrollmessungen durchgeführt. Zum einen wurden $\mathrm{zu}$ einer Vesikellösung des Lysinollipids in $40 \mathrm{~mm}$ MES/200 mM KCl Methanol (400 mM) und Salzsäure (0,1 mM) zugegeben, um auszuschließen, dass die Zunahme der hydrodynamischen Radien durch Wechselwirkung mit diesen Substanzen zustande kommen. Methanol wird bei der Hydrolyse von TMOS zu Kieselsäure in Salzsäure freigesetzt und beide Substanzen sind somit in den entsprechenden Konzentrationen in allen Proben zusätzlich zu Si $(\mathrm{OH})_{4}$ enthalten. Bei dieser Messung konnte weder beim hydrodynamischen Radius noch in der Signalintensität eine signifikante Veränderung beobachtet werden. Zum anderen wurde frisch hergestellte Monokieselsäure (100 mM) zu Puffer (50 mM MES pH 6,0) zugegeben und das System mittels DLS vermessen, um sicherzustellen, dass im Puffer in Abwesenheit der Aminolipide keine Silicapolymerisation stattfindet. Hier wurde aufgrund des Fehlens Lichtstreuender Partikel sowohl vor als auch nach der Zugabe lediglich ein extrem schwaches Signal detektiert, das sich innerhalb des beobachteten Zeitfensters von zehn Minuten nicht änderte. Somit kann davon ausgegangen werden, dass die Zunahme der Radien ein Ergebnis der Reaktion von $\mathrm{Si}(\mathrm{OH})_{4}$ mit Aminolipidvesikeln ist. Nachdem mittels der $\beta$-Molybdatmethode nachgewiesen wurde, dass in Suspensionen von Aminolipidvesikeln Präzipitation stattfand, kann davon ausgegangen werden, dass die Vergrößerung der hydrodynamischen Radien der Vesikel durch die Silicabildung verursacht wurde. Weitestgehend war die Vergrößerung umso stärker ausgeprägt, je mehr Silica mittels $\beta$-Molybdattest im entsprechenden System detektiert wurde. Dies verstärkt die Erkenntnis, dass die strukturellen Unterschiede in den Kopfgruppen der Aminolipide auch unterschiedliches Verhalten bei Präzipitationsreaktionen bedingen.

Um einen visuellen Eindruck der bei den DLS-Messungen entstehenden Reaktionsprodukte $\mathrm{zu}$ bekommen, wurden die Silicapräzipitate rasterelektronenmikroskopisch untersucht. Hierzu wurden Proben direkt aus der Lichtstreuungsmesszelle entnommen, auf einen SEM-Probenträger getropft und nach Eintrocknen vermessen. Die Präzipitate konnten dabei weder zentrifugiert noch mit Wasser gewaschen werden, so dass überschüssige Kieselsäure nicht entfernt wurde. In Abbildung 5.4 sind Aufnahmen 
dargestellt, die bei Messung der Probe von Lysinollipid in $40 \mathrm{~mm} \mathrm{MES/200} \mathrm{mm} \mathrm{KCl}$ entstanden. Die Probe ist stark heterogen, es finden sich unterschiedliche Objekte. In Aufnahmen A und B sind kugelförmige Strukturen erkennbar, die ungleichmäßig geformt sind und Durchmesser von 1 - $6 \mu \mathrm{m}$ besitzen und damit in der Größenordnung liegen, die mittels dynamischer Lichtstreuung ermittelt wurde (Abbildung 5.3). Daneben liegt viel Material in kleineren Größen vor sowie einige deutlich größere Strukturen. Aufnahme C zeigt die Aggregation einzelner Partikel. Die Proben, die mit den anderen Aminolipiden entstanden, waren ebenfalls stark inhomogen.
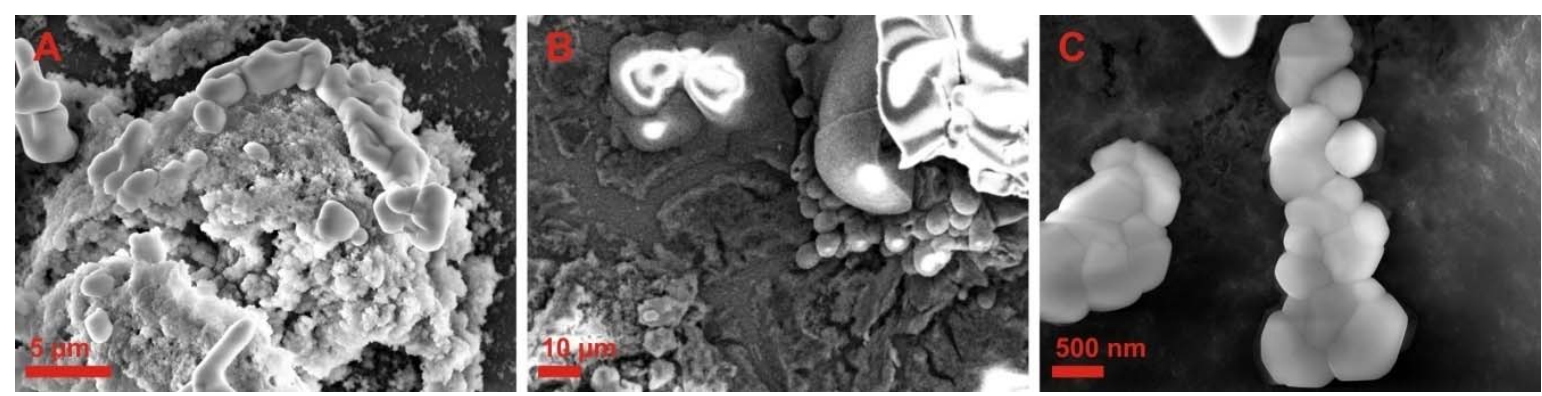

Abbildung 5.4 SEM-Aufnahmen von Silicapräzipitaten, entstanden durch Zugabe von $100 \mathrm{mM} \mathrm{Si}(\mathrm{OH})_{4} \mathrm{zu}$ Vesikeln des Lysinollipids ( $c=1 \mathrm{mM}$ ) in $40 \mathrm{mM}$ MES/200 mM KCl pH 6,0. Reaktionszeit: $13 \mathrm{~min}$.

Im Vergleich dazu wurden auch Präzipitate rasterelektronenmikroskopisch untersucht, die nach zehnminütiger Inkubation zentrifugiert und im Anschluss mehrmals mit Wasser gewaschen wurden, um überschüssige Kieselsäure zu entfernen. Diese Vorgehensweise entspricht der beim zuvor vorgestellten $\beta$-Molybdattest. Hierzu wurden Vesikel mittels Extrusion durch Polycarbonatmembranen mit Porendurchmessern von $400 \mathrm{~nm}$ hergestellt und mit Monokieselsäure versetzt. In den in Abbildung 5.5 dargestellten SEM-Aufnahmen ist Silica zu sehen, das bei diesen Experimenten präzipitiert wurde.
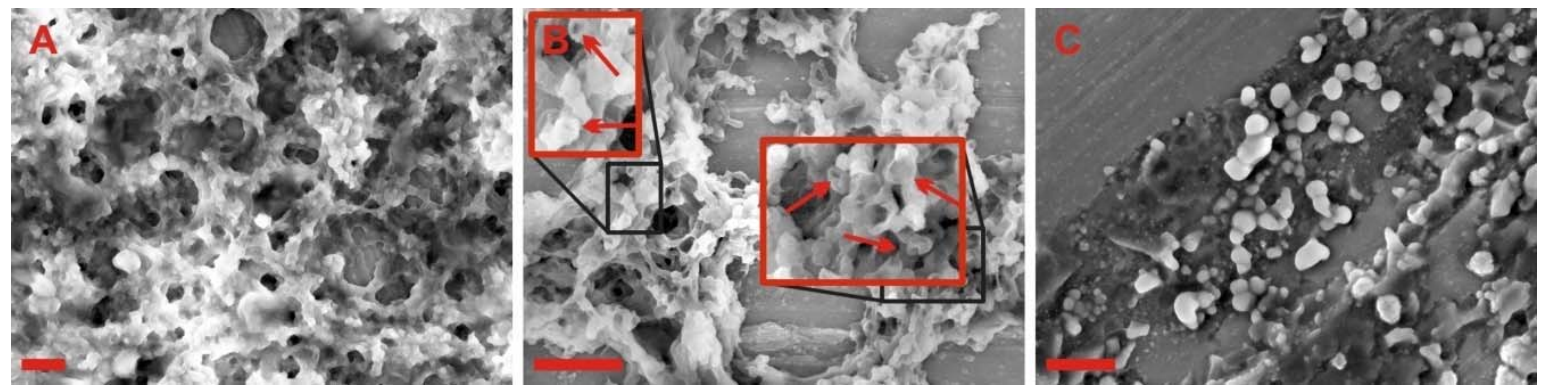

Abbildung 5.5 Rasterelektronenmikroskopische Aufnahmen von Silica. Zu Vesikeln des Lysinollipids in $40 \mathrm{mM} \mathrm{MES} / 200 \mathrm{mM} \mathrm{KCl} \mathrm{pH} \mathrm{6,0} \mathrm{wurden} 25 \mathrm{mM} \mathrm{Si}(\mathrm{OH})_{4}$ zugegeben und nach ca. 10 min zentrifugiert und mehrmals mit Wasser gewaschen. A $c_{\text {Lipid }}: 0,08 \mathrm{mM}, \mathbf{B} c_{\text {Lipid }}: 0,13 \mathrm{mM}$ und $\mathbf{C} c_{\text {Lipid }}: 0,8 \mathrm{mM}$. Maßstabsbalken: $1 \mu \mathrm{m}$. 
Nur im Ansatz zeichnen sich kugelförmige Strukturen ab, die mit einem mittleren Durchmesser von ca. $160 \mathrm{~nm}$ deutlich kleiner sind, als die in Abbildung 5.4 auftretenden. Die Vesikel waren jedoch aller Wahrscheinlichkeit nach hier größer, da für die Herstellung größere Poren verwendet wurden. Die Proben haben ein homogeneres Erscheinungsbild, als die zuvor gezeigten. Teilweise wirken die Strukturen wie zerstörte dünne, labile Silicaschalen (Pfeile). Die Behandlung der Präzipitate scheint Einfluss auf die Strukturen zu haben. Die Form der Vesikel, die als Template für die Silicabildung dienen, wird in jedem Fall nur ansatzweise im präzipitierten $\mathrm{SiO}_{2}$ wiedergefunden.

\subsection{Diskussion}

\section{Eigenschaften der Aminolipide}

Bevor sowohl die Aggregatbildung der Aminolipide als auch die mit ihnen durchgeführten Silicapräzipitationen diskutiert werden, wird auf ihr Säure-Base-Verhalten eingegangen. Dies ist im Hinblick auf die Erklärung der Ergebnisse von Interesse, da der Protonierungszustand und damit zusammenhängende Ladungen in den Kopfgruppen die Bildung mizellarer und vesikulärer Strukturen beeinflussen. Auch die Wechselwirkungen der in der Kopfgruppe befindlichen Aminogruppe/n mit Kieselsäure, die die Bildung von Silica bewirken, könnte von elektrostatischen und sterischen Parametern abhängen.

Der Unterschied zwischen den Aminolipiden liegt in der $N$-Methylierung beziehungsweise der Anzahl der Aminogruppen. Diese strukturellen Variationen führen zu Abweichungen der jeweiligen Säurestärke der konjugierten Säure, welche mit dem $\mathrm{pK}_{\mathrm{s}}$-Tool von SPARC $^{70}$ ermittelt wurden. Sie ist zusammen mit der mittleren Ladung $\bar{z}$ und dem Protonierungsgrad $(z / \%)$ (tatsächliche Ladung/maximal mögliche Ladung) in Tabelle 5.2 aufgeführt. Hier finden sich weiterhin Angaben zur Anzahl der Aminoheiten pro Molekül $N_{\mathrm{N}}$. Es wird deutlich, dass zwischen primärem und sekundärem Aminolipid kein großer Unterschied bezüglich der Basizität besteht, die $\mathrm{pK}_{\mathrm{s}}$-Werte sind mit 8,68 und 8,95 ähnlich. Dagegen ist sie im tertiären Aminolipid deutlich reduziert, der $\mathrm{pK}_{\mathrm{s}}$-Wert beträgt hier lediglich 5,38. Daraus ergibt sich auch eine durchschnittliche Ladung von 0,2 beziehungsweise ein Protonierungsgrad von nur $19 \%$ bei $\mathrm{pH}$ 6,0, während pAL und sAL unter den gleichen Bedingungen nahezu vollständig protoniert sind. Für das quartäre Aminolipid gibt es keine $\mathrm{pK}_{\mathrm{s}}-$ Werte, es ist unter allen Bedingungen positiv geladen, da das Stickstoffatom vierfach alkyliert ist. 
Tabelle 5.2 Eigenschaften der Aminolipide: primäres (pAL), sekundäres (sAL), tertiäres (tAL), quartäres (qAL) Aminolipid und Lysinollipid (LysinolL) bei pH 6,0.

\begin{tabular}{cccccc}
\hline Abkürzung & $\boldsymbol{N}_{\mathbf{N}}$ & $\mathbf{p K}_{\mathbf{s} \mathbf{1}}$ & $\mathbf{p K}_{\mathbf{s ~} 2}$ & $\overline{\mathbf{z}}$ & $\boldsymbol{z} / \%$ \\
\hline $\mathrm{pAL}$ & 1 & 8,68 & - & 1,0 & 99,8 \\
\hline $\mathrm{sAL}$ & 1 & 8,95 & - & 1,0 & 99,9 \\
\hline $\mathrm{tAL}$ & 1 & 5,38 & - & 0,2 & 19,0 \\
\hline $\mathrm{qAL}$ & 1 & - & - & 1,0 & 100,0 \\
\hline LysinolL & 2 & 9,14 & 10,33 & 2,0 & 99,9 \\
\hline
\end{tabular}

Das Lysinollipid besitzt eine andere Struktur als die anderen Aminolipide, da es zwei primäre Aminoeinheiten enthält, die leicht unterschiedliche $\mathrm{pK}_{\mathrm{s}}$-Werte von 9,14 beziehungsweise 10,33 besitzen. Die Verbindung ist, wie pAL und sAL, bei pH 6,0 vollständig protoniert, weist aber im Gegensatz zu ihnen eine mittlere Ladung von 2,0 auf. Es bleibt bei allen Substanzen zu bedenken, dass sich die Aussagen zu Ladungen und $\mathrm{pK}_{\mathrm{s}^{-}}$ Werten lediglich auf ein isoliert betrachtetes Molekül beziehen. Die erläuterten Eigenschaften sind in Abbildung 5.6 veranschaulicht.

A

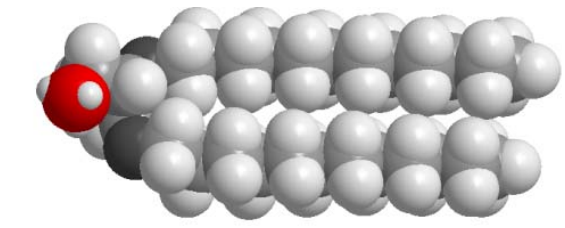

C

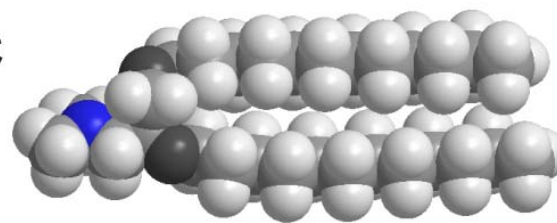

B

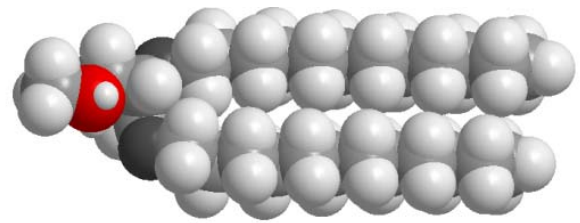

$\mathrm{D}$

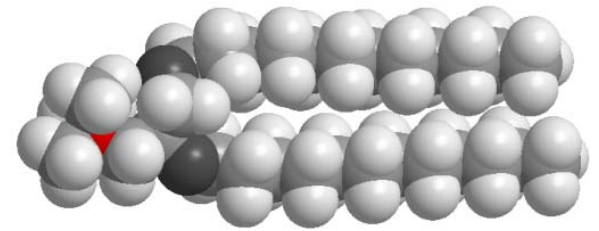

E

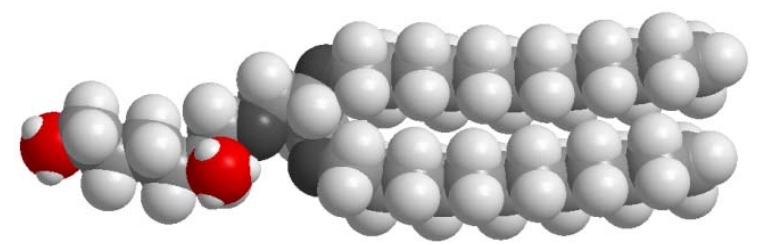

Abbildung 5.6 Kalottenmodelle der Aminolipide: A primäres, B sekundäres, C tertiäres, D quartäres Aminolipid und E Lysinollipid mit Ladung bei pH 6,0. $\odot \mathrm{C}, \bigcirc \mathrm{N}, \bigcirc \mathrm{N}^{+}, \mathcal{O}, \mathcal{\odot} \mathrm{H}$ 


\section{Aggregatbildung der Aminolipide}

Die Untersuchung der Lipidsuspensionen wurde mittels dynamischer Lichtstreuung durchgeführt. Dabei wurde ermittelt, dass alle Aminolipide Aggregate bilden. In einem Puffer hoher Ionenstärke (25 mM MES / $500 \mathrm{mM} \mathrm{KCl)} \mathrm{treten} \mathrm{ausschließlich} \mathrm{Vesikel} \mathrm{auf.} \mathrm{Es}$ kann dementsprechend davon ausgegangen, dass die Packungsparameter der Amphiphile (G1. 5-1) unter den gegebenen Bedingungen im Bereich von $0,5 \leq P \leq 1$ liegen. ${ }^{144}$ Alle Verbindungen, außer dem primären Aminolipid, aggregieren in großen unilamellaren Vesikeln (large unilamellar vesicles, LUVs) mit mittleren Durchmessern von (130 - 220) nm (Abbildung 5.1), was im erwarteten Größenbereich liegt, da die Suspension durch Poren eines Durchmessers von $200 \mathrm{~nm}$ extrudiert wurden. ${ }^{145}$ Dagegen treten beim primären Aminolipid sehr große Vesikel $(d=780 \mathrm{~nm})$ auf. Es ist zu beachten, dass die Präparation in diesem Fall nicht bei Raumtemperatur stattfand, sondern bei $63^{\circ} \mathrm{C}$, da sich bei Raumtemperatur keine homogenen Lipidfilme bildeten und diese mit Puffer nicht rehydratisiert werden konnten, um Suspensionen herzustellen. Daraus ist abzuleiten, dass die Hauptphasenumwandlungstemperatur, die für die Aminolipide nicht bekannt ist, im Fall des primären Aminolipids zumindest über Raumtemperatur liegen muss. Crommelin ${ }^{146}$ und Carmona-Ribeiro ${ }^{147,148}$ berichteten, dass positiv geladene Vesikel nicht stabil sind und sich zu größeren Aggregaten zusammenlagern oder fusionieren. Tatsächlich wurden bei Lichtstreuungsmessungen der Aminolipidvesikel schon nach einigen Stunden deutlich vergrößerte Aggregate detektiert. Es kann davon ausgegangen werden, dass Aggregations- und/oder Fusionsprozesse durch die erhöhte Temperatur bei der Vesikelpräparation im Fall des primären Aminolipids noch beschleunigt wurden und aus diesem Grund bereits innerhalb der kurzen Zeit von ca. zwanzig Minuten zwischen Präparation und Messung größere Aggregate gebildet wurden.

Differenzierter müssen die Ergebnisse betrachtet werden, die in einem Puffer niedrigerer Ionenstärke (50 mM MES) ermittelt wurden (Abbildung 5.1). Hier liegen das primäre, sekundäre, tertiäre sowie das quartäre Aminolipid ausschließlich in lamellaren Aggregaten vor. Auch in diesem Fall liegen wahrscheinlich Packungsparameter von $0,5 \leq P \leq 1$ vor. Die Durchmesser der Vesikel liegen beim primären, sekundären beziehungsweise tertiären Aminolipid mit $(170$ - 180) nm in der erwarteten Größenordnung. Die Durchmesser der vom quartären Aminolipid gebildeten Vesikeln $(d=100 \mathrm{~nm})$ sind kleiner, jedoch ebenfalls im bekannten Bereich. ${ }^{81}$ Dahingegen bildet das Lysinollipid in diesem Puffer zusätzlich zu den LUVs mizellare Aggregate mit Durchmessern unter $5 \mathrm{~nm} .{ }^{143}$ Offensichtlich ist die Geometrie der Moleküle in Abwesenheit von Kaliumchlorid verändert, der 
Packungsparameter $P$ der Moleküle ist vermutlich kleiner 0,5. Eine Erhöhung der Ionenstärke bewirkte eine deutliche Verschiebung von mizellaren hin $\mathrm{zu}$ vesikulären Strukturen (Abbildung 5.1 E). Der Packungsparameter und damit die Aggregatform von Amphiphilen wird von Faktoren wie Temperaturänderungen, pH-Wert oder Ionenkonzentrationen beeinflusst. ${ }^{149,150}$ Wahrscheinlich ist, dass im vorliegenden Fall die erhöhte Ionenstärke die positive Ladung in den Kopfgruppen elektrostatisch abschirmt, wodurch die elektrostatische Repulsion zwischen den Kopfgruppen vermindert wird und woraus eine Verkleinerung der effektiven Kopfgruppengröße resultiert. ${ }^{144,151}$ Der Packungsparameter wird durch eine Abnahme der Fläche der Kopfgruppe $a_{0}$ vergrößert (vgl. Gleichung 5-1), wodurch die Bildung lamellarer Strukturen begünstigt wird. Damit im Einklang steht, dass beim Lysinollipid in einem Puffer mittlerer Ionenstärke (40 mM MES / $200 \mathrm{mM} \mathrm{KCl)} \mathrm{weniger} \mathrm{Moleküle} \mathrm{in} \mathrm{Mizellen} \mathrm{aggregiert} \mathrm{sind,} \mathrm{als} \mathrm{dies} \mathrm{in}$ Abwesenheit von $\mathrm{KCl}$ der Fall ist. Es treten jedoch nicht immer ausschließlich Vesikel auf, was erst bei noch weiterer Erhöhung der KCl-Konzentration auf $500 \mathrm{~mm}$ beobachtet wird. Daraus kann folgender Zusammenhang für den Packungsparameter des Lysinollipids abgeleitet werden:

$P_{500 \mathrm{~mm} \mathrm{KCl}}>P_{200 \mathrm{~mm} \mathrm{KCl}}>P_{0 \mathrm{~mm} \mathrm{KCl}}$.

\section{Silicapräzipitationen mit Vesikeln der Aminolipide}

In den bei Diatomeen vorkommenden Polyaminen und Silaffinen treten Aminogruppen verschiedener $N$-Methylierungsgrade auf. Es wurden sowohl primäre, sekundäre, tertiäre als auch quartäre Aminoeinheiten entdeckt. ${ }^{55}$ Dabei gibt es speziesspezifische Unterschiede im $N$-Methylierungsmuster. ${ }^{56}$ Beispielsweise kommen in den zu den Pennales gehörenden Diatomeen Cylindrotheca fusiformis, außer an den Molekültermini, die aus tertiären Aminen bestehen, ausschließlich sekundäre Aminoeinheiten vor. Dahingegen wurden in den, den Centrales zugeordneten, bisher untersuchten Diatomeen, wie Stephanopyxis turris oder Coscinodiscus wailesii, Polyamine mit stärker ausgeprägter $N$ Methylierung identifiziert. ${ }^{63}$ Es wird davon ausgegangen, dass die spezifische Zusammensetzung an Polyaminen die bei jeder Spezies unterschiedliche Musterung der Silicazellwände mitbeeinflusst. ${ }^{32}$ Jedoch ist der $N$-Methylierungsgrad der Polyamine nicht das einzige Strukturmerkmal, das die Silicabildung dirigiert. Auch die Kettenlänge, die Position sekundärer, tertiärer und quartärer Gruppen sowie die Alkyleinheit zwischen den Stickstoffatomen spielen eine Rolle, wie nicht zuletzt in Kapitel 4 gezeigt werden konnte. Genau diese Komplexität macht es schwierig, einzelne Parameter zu untersuchen, um die 
Silicabildung Schritt für Schritt aufzuklären. Es ist nicht nur von Interesse, den molekularen Mechanismus $\mathrm{zu}$ verstehen, mit dem eine einzelne Zelle das morphogenetische Programm für die Bildung von anorganischem Material ausführt, sondern die Erkenntnisse auch im Bereich der Herstellung von Nanomaterialien anzuwenden. In vielen Arbeiten wurde versucht, sich das Wissen über die in Diatomeen ablaufenden Silicabildungsprozesse zunutze zu machen und dadurch komplexe, neue Materialien auf einer schnellen Zeitskala unter milden, physiologischen Bedingungen und auf einfache Art und Weise herzustellen. ${ }^{152-158}$

Der hier mit den Aminolipiden verfolgte biomimetische Ansatz versucht einen Beitrag zu beiden Bereichen zu leisten. Die Vorgabe der Aggregatform und -größe ermöglicht die Untersuchung des direkten Einflusses des Säure-Base-Verhaltens, der sterischen Parameter sowie der Anzahl an Aminoeinheiten auf die Silicabildung. In anderen Forschungs$\operatorname{arbeiten}^{102,129}$ und bei der Verwendung der Polyamine (Kapitel 4) war dies nicht möglich, da die aufgezählten Faktoren dort auch die vor der Präzipitation stattfindende Aggregation beeinflussen und somit nicht strikt auf die Silicapolymerisation bezogen werden können. Zudem sollte getestet werden, inwiefern vesikuläre Aggregate der Aminolipide als Template für Silicapräzipitationen einsetzbar sind und ob und wie gut ihre Form abgebildet wird.

Um einschätzen zu können, wie die Silicabildung an der Oberfläche der Vesikel abläuft, wurden zunächst Berechnungen dazu durchgeführt, welche Silicamengen erwartet werden, wenn sich Siliciumdioxid in Form eckenverknüpfter Tetraeder in einer Monoschicht um die Vesikel herum anlagert. Für die Berechnung wurden die mittels Lichtstreuung ermittelten Durchmesser der Vesikel (Abbildung 5.1) verwendet und die Annahme getroffen, dass ihre Außenoberfläche $56 \%$ der Gesamtoberfläche ausmacht. Weiterhin wird davon ausgegangen, dass alle eingesetzten Lipidmoleküle in Vesikeln angeordnet sind. Über die Querschnittsflächen der Moleküle wurden die Gesamtaußenoberflächen, an denen Reaktionen ablaufen können, pro Reaktionsansatz ermittelt. Für das Lysinollipid ist die Fläche pro Molekül aus Filmwaagemessungen bekannt (Abbildung 6.16 A), für die anderen vier Verbindungen wurde diese mit Hilfe der Software ChemBio 3D Ultra abgeschätzt. In Tabelle 5.3 werden die Ergebnisse der Berechnungen aufgeführt sowie zum Vergleich dazu die experimentellen Ergebnisse. Zunächst ist festzustellen, dass die berechneten und gemessenen Siliciummassen in einem ähnlichen Größenbereich liegen. 
Tabelle 5.3 Daten zur Molekülfläche der Aminolipide sowie theoretische und experimentelle Siliciummassen in Silicapräzipitaten. Die Siliciummassen wurden mittels $\beta$-Molybdatmethode in $50 \mathrm{mM} \mathrm{MES} \mathrm{pH} 6,0$ bestimmt (vgl. Abbildung 5.2).

\begin{tabular}{cccc}
\hline Abkürzung & $\begin{array}{c}\text { Fläche pro } \\
\text { Molekül } / \AA^{2}\end{array}$ & $\begin{array}{c}\text { berechnete } \\
\boldsymbol{m}_{\mathbf{S i}} / \boldsymbol{\mu g}\end{array}$ & $\begin{array}{c}\text { ermittelte } \\
\boldsymbol{m}_{\mathrm{Si}} \boldsymbol{I} \boldsymbol{\mu g}\end{array}$ \\
\hline $\mathrm{pAL}$ & 15 & 4 & 22 \\
\hline $\mathrm{SAL}$ & 23 & 6 & 28 \\
\hline $\mathrm{tAL}$ & 24 & 6 & 9 \\
\hline $\mathrm{qAL}$ & 31 & 8 & 59 \\
\hline LysinolL & 51 & 13 & 76 \\
\hline
\end{tabular}

Zunächst ist festzustellen, dass die berechneten und gemessenen Siliciummassen im gleichen Größenbereich liegen, im Fall von pAL, sAL, qAL und LysinolL sind die berechneten Werte um einen Faktor 5 - 7 kleiner. Da die Berechnung auf der Annahme beruht, dass eine Silicamonoschicht um die Vesikel gebildet wird, kann davon ausgegangen werden, dass sich tatsächlich nur wenige Schichten $\mathrm{SiO}_{2}$ um die Vesikel gelegt haben. Dagegen scheinen die Oberflächen der Vesikel des tertiären Aminolipids weniger stark mit Silica bedeckt zu sein, die berechnete Masse ist ähnlich groß, wie die bei den Messungen bestimmte.

Um Erklärungen für die in Kapitel 5.3 vorgestellten Ergebnisse zu finden, müssen diese differenziert betrachtet werden. Zunächst wird der Einfluss der Ionenstärke auf die Silicabildung betrachtet. Die Ergebnisse aus Abbildung 5.2 verdeutlichen, dass sich eine hohe Ionenstärke negativ auf die Präzipitationsmenge auswirkt. In fast allen Fällen wird in Anwesenheit von $500 \mathrm{mM} \mathrm{KCl}$ weniger Silica gebildet, als in Abwesenheit von Kaliumchlorid. Dies kann dadurch begründet werden, dass die positive Ladung der Aminogruppen elektrostatisch durch die Ionen abgeschirmt wird. Laut Mizutani ${ }^{42}$, Iler ${ }^{35}$ und Kröger ${ }^{36}$ beruht die Polymerisation von Silica in Anwesenheit von Aminen auf der Reaktion der Aminogruppen mit Kieselsäure, auch wenn der genaue Mechanismus nicht geklärt ist. Eine Behinderung der Reaktion aufgrund der Abschirmung positiver Ladung weist darauf hin, dass die Protonierung der Aminogruppen wichtig ist.

Die Ionenstärke beeinflusst bei den Experimenten nicht nur die Reaktion, sondern schon zuvor die Bildung von Vesikeln. Um zu klären, wie sich die Aggregation der Moleküle auf die Präzipitation auswirkt, werden im Folgenden die mit dem Lysinollipid ermittelten Daten diskutiert. Die Daten zeigen, dass die größte Silicamenge präzipitiert wurde, wenn die Vesikel bei hoher Ionenstärke $\left(c_{\mathrm{KCl}}=500 \mathrm{mM}\right)$ hergestellt wurden und anschließend die 
Ionenstärke verringert wurde $\left(c_{\mathrm{KCl}}=200 \mathrm{mM}\right)$ (Abbildung 5.2). Dies scheint zunächst im Widerspruch $\mathrm{zu}$ der oben aufgestellten Hypothese $\mathrm{zu}$ stehen. Jedoch zeigten die Lichtstreuungsmessungen, dass ohne $\mathrm{KCl}$ überwiegend mizellare Strukturen gebildet werden (Abbildung 5.1 E). Eine mögliche Erklärung hierfür ist, dass vesikuläre Strukturen deutlich besser geeignet sind, Präzipitation zu induzieren als mizellare Aggregate. Dieser Faktor hat hier einen stärkeren Einfluss, als die negative Wirkung der Ladungsabschirmung bei erhöhter Ionenkonzentration $\left(c_{\mathrm{KCl}}=200 \mathrm{mM}\right)$, weshalb die Präzipitationsmenge höher ist als in Abwesenheit von $\mathrm{KCl}$. Die weitere Erhöhung der Ionenstärke auf $c_{\mathrm{KCl}}=500 \mathrm{mM}$ führt zwar dazu, dass noch mehr Moleküle in lamellarer Struktur aggregieren und keine Mizellen mehr auftreten (Abbildung $5.1 \mathrm{E}$ ), trotzdem wird hier weniger Silica gebildet als bei $c_{\mathrm{KCl}}=200 \mathrm{mM}$. Bei dieser Ionenstärke scheint der ladungsabschirmende Einfluss der Ionen zu überwiegen.

Ein weiterer Parameter, der in die Betrachtungen einbezogen wird, ist das Säure-BaseVerhalten der Aminolipide. Vergleicht man die in Tabelle 5.2 aufgeführten mittleren Ladungen der Verbindungen bei pH 6,0, so wird deutlich, dass sich diese unterscheiden. Sie geben jedoch lediglich die Ladung pro Molekül wieder. Für eine bessere Einschätzung der an den Oberflächen der Vesikel vorliegenden Ladungsverhältnisse, ist es jedoch nötig, auch die jeweilige Fläche, auf welche sich diese Ladung verteilt mit einzukalkulieren. Aus diesem Grund wird eine Abschätzung der Ladungsdichte an der Oberfläche der jeweiligen Vesikel vorgenommen. Hierzu wird zunächst über die mittlere Ladung $\bar{z}$ (Tabelle 5.2) die Ladung $Q$ pro Molekül laut Gl. 5-2 berechnet:

$$
Q=\bar{z} e
$$

wobei $e$ für die Elementarladung $\left(1,6022 \cdot 10^{-19} \mathrm{C}\right)$ steht. Im Anschluss wird die Ladungsdichte $D$ unter Einbeziehung der Querschnittsflächen der Moleküle (vgl. Tabelle 5.3) berechnet:

$$
D=\frac{Q}{\text { Fläche pro Molekül }}
$$

Die Ergebnisse für die Aminolipide pAL, sAL, tAL, qAL und Lysinollipid sind in Tabelle 5.4 zusammen mit den ermittelten Siliciummassen $m_{\mathrm{Si}}$ (Abbildung 5.2) aufgeführt. 
Tabelle 5.4 Ergebnisse der Berechnung der Ladung $Q$ pro Molekül sowie der Ladungsdichte $D$ für Vesikel der Aminolipide im Vergleich mit den mittels $\beta$-Molybdattest detektierten Siliciummassen der Präzipitationen in $50 \mathrm{mM}$ MES pH 6,0.

\begin{tabular}{cccc}
\hline Abkürzung & $\boldsymbol{Q} / \mathbf{1 0}^{-19} \mathbf{C}$ & $\boldsymbol{D} /\left(\mathbf{C} / \mathbf{m}^{2}\right)$ & ermittelte $\boldsymbol{m}_{\mathbf{S i}} / \boldsymbol{\mu g}$ \\
\hline $\mathrm{pAL}$ & 1,6 & 1,1 & 22 \\
\hline $\mathrm{SAL}$ & 1,6 & 0,7 & 28 \\
\hline $\mathrm{tAL}$ & 0,3 & 0,1 & 9 \\
\hline $\mathrm{qAL}$ & 1,6 & 0,5 & 59 \\
\hline LysinolL & 3,2 & 0,6 & 76 \\
\hline
\end{tabular}

Das tertiäre Aminolipid hat einen niedrigen $\mathrm{pK}_{\mathrm{s}}$-Wert und ist lediglich $\mathrm{zu}$ ca. $19 \%$ protoniert ( Tabelle 5.2), was sich auch in einer im Vergleich zu den anderen Aminolipiden niedrigen Ladungsdichte von $0,1 \mathrm{C} / \mathrm{m}^{2}$ äußert. Alle anderen Substanzen sind zwar (fast) maximal positiv geladen (Tabelle 5.2), jedoch unterscheiden sich ihre Ladungsdichten (Tabelle 5.4) aufgrund der abweichenden Querschnittsflächen ihrer Kopfgruppen. Die Ladungsdichte des primären Aminolipids ist demnach deutlich am größten, gefolgt von der des sekundären Aminolipids und der des Lysinollipids. Das quartäre Aminolipid hat mit $0,5 \mathrm{C} / \mathrm{m}^{2}$ eine kleinere Ladungsdichte als die eben aufgeführten, sie ist jedoch deutlich höher als die des tertiären Aminolipids.

Ein Vergleich der Ladungsdichten mit den Silicamengen, die in Versuchen präzipitiert wurden (Tabelle 5.4), deutet zunächst nicht auf einen Zusammenhang zwischen diesen Parametern hin. Es ist jedoch nötig, die theoretisch ermittelten $\mathrm{pK}_{\mathrm{s}}$-Werte (Tabelle 5.2), auf denen alle folgenden Berechnungen zu Protonierung und Ladung basieren, kritisch zu betrachten. Bei der Berechnung der $\mathrm{pK}_{\mathrm{s}}$-Werte kann die Aggregation der Moleküle nicht berücksichtigt werden. Es ist jedoch bekannt, dass sich die Ladungen durch die räumliche Nähe, die durch Akkumulation bedingt wird, beeinflussen. ${ }^{159}$ So nimmt beispielsweise der $\mathrm{pK}_{\mathrm{s}}$-Wert von Fettsäuren deutlich $\mathrm{zu}$, wenn sie sich $\mathrm{zu}$ lamellaren oder kristallinen Aggregaten zusammenlagern. ${ }^{149,160}$ Der $\mathrm{pK}_{\mathrm{s}}$-Wert von Stearylamin in Vesikeln hingegen ist um eine Einheit kleiner als der $\mathrm{pK}_{\mathrm{s}}$-Wert der Monomere. ${ }^{159}$ Dies wurde unter anderem durch elektrostatische Effekte und die geringe Polarität der Lipidmatrix erklärt. ${ }^{159}$ Demnach ist $\mathrm{zu}$ erwarten, dass auch die effektiven $\mathrm{pK}_{\mathrm{s}}$-Werte der Aminolipide in lamellaren Strukturen niedriger sind, als die $\mathrm{pK}_{\mathrm{s}}$-Werte der Monomere, die in Tabelle 5.2 aufgeführt sind. Weiterhin ist es wahrscheinlich, dass sich die Ladungen umso stärker gegenseitig beeinflussen, je weniger Abstand zwischen den Aminoeinheiten ist, was 
bedeuten würde, dass im Fall des primären Aminolipids die Erniedrigung des $\mathrm{pK}_{\mathrm{s}}-$ Wertes am deutlichsten ausfallen müsste und beim Lysinollipid am geringsten. Eine Quantifizierung der beschriebenen Effekte ist nicht möglich, doch resultierend kann angenommen werden, dass durch die gegenläufigen Trends - Anstieg der Ladungsdichte (bei gleicher Protonierung) und Anstieg der $\mathrm{pK}_{\mathrm{s}}$-Wert-Erniedrigung mit Abnahme der Molekülfläche - im Fall des primären und sekundären Aminolipids sowie des Lysinollipids eine ähnliche Ladungsdichte auf der Vesikeloberfläche vorliegt, die insgesamt niedriger ist, als in Tabelle 5.4 aufgeführt. Beim tertiären Aminolipid ist die Ladungsdichte bei $\mathrm{pH}$ 6,0 aufgrund des niedrigen $\mathrm{pK}_{\mathrm{s}}$-Wertes vermutlich deutlich niedriger als bei den vier anderen Verbindungen. Die beschriebenen Überlegungen beziehen sich nicht auf das quartäre Aminolipid, dass aufgrund der Methylierung unabhängig von pH-Wert, Aggregation oder anderen Bedingungen permanent geladen ist. Somit kann davon ausgegangen werden, dass Vesikel des quartären Aminolipids insgesamt die höchste Ladungsdichte besitzen.

Unter Berücksichtigung der eben erläuterten Erkenntnisse über die Ladungsdichten, kann die Korrelation zwischen Molekülfläche und experimentell ermittelten Silicamengen (Tabelle 5.3) bei primärem, sekundären Aminolipid und Lysinollipid erklärt werden. In allen Experimenten war die Konzentration der Substanzen gleich groß, doch die Reaktionsflächen unterschieden sich aufgrund der unterschiedlichen Molekülflächen. Dies ist der Grund, warum mit Lysinollipidvesikeln mehr Silica gebildet wurde, als mit Vesikeln des sekundären Aminolipids, welche wiederum eine größere Silicamenge präzipitierten als die des primären Aminolipids, obwohl die Ladungsdichte wahrscheinlich in allen Vesikeln ähnlich ist (siehe oben). Dahingegen bewirkt die hohe Ladungsdichte der Vesikel des quartären Aminolipids, die durch die permanente Ladung bedingt ist, die Präzipitation einer Silicamenge, die im Verhältnis größer ist. Im Fall des tertiären Aminolipids verursacht die niedrigere Ladungsdichte, die Resultat des niedrigen $\mathrm{pK}_{\mathrm{s}}$-Wertes ist, eine Verminderung der tatsächlich präzipitierten Silicamenge im Vergleich zur erwarteten.

Ein weiterer Grund für die geringe Silicamenge könnte sterische Hinderung bei der Reaktion mit Kieselsäure bedingt durch die drei Methylgruppen sein, jedoch müsste dieser sterische Einfluss dann auch beim quartären Aminolipid postuliert werden (siehe Abbildungen 5.6 C und D), was nicht der Fall ist. Eine weiterer Unterschied zu einem Teil der anderen Aminolipide ist die Aggregatverteilung. Wie aus Abbildung 5.1 C ersichtlich ist, liegt ein Teil der tertiären Aminolipidmoleküle in Abwesenheit von Kaliumchlorid, wie auch beim Lysinollipid, in mizellarer Form vor. Doch im Gegensatz zum Lysinollipid 
wurde mit dem tertiären Aminolipid deutlich weniger Silica gebildet als erwartet. Folgerung daraus ist, dass der Protonierungsgrad und damit die Ladungsdichte an der Reaktionsfläche eine wesentliche Rolle spielt. Je mehr positiv geladene Aminogruppen an der Oberfläche für die Reaktion mit Kieselsäure zur Verfügung stehen, desto mehr Silica wird gebildet.

Es bleibt zu klären, warum bei Verwendung von Vesikeln des quartären Aminolipids verhältnismäßig mehr Silica gebildet wurde, als mit beispielsweise primärem oder sekundären Aminolipid, die laut der Werte in Tabelle 5.2 alle nahezu vollständig protoniert sind. Es ist bekannt, dass sich der $\mathrm{pK}_{\mathrm{s}}$-Wert von Amphiphilen, die in Mizellen oder anderen Strukturen aggregiert sind, von dem der monomeren Moleküle unterscheidet. ${ }^{159}$ Der letzte Faktor, dessen Einfluss an dieser Stelle diskutiert wird, ist die Anzahl der Aminogruppen pro Molekül. Das Verhältnis von ermittelter zu berechneter Siliciummasse für das primäre Aminolipid und das Lysinollipid, die beide primäre Aminogruppen beinhalten, ist mit jeweils ca. 1,3 identisch. Daraus könnte gefolgert werden, dass sich der Unterschied zwischen den beiden Verbindungen, das heißt die Anzahl primärer Aminogruppen pro Molekül, nicht auf die Präzipitationsmenge auswirkt. Jedoch darf nicht außer Acht gelassen werden, dass, wie oben ausführlich erläutert wurde, in Abwesenheit von Kaliumchlorid neben Vesikeln auch Mizellen vorliegen und bei veränderter Präparation der Suspensionen deutlich mehr Silica gebildet wurde, als berechnet. Das Verhältnis der Werte bei $c_{\mathrm{KCl}}=200 \mathrm{mM}$ beträgt hier (ermittelt/berechnete Siliciummasse) 2,3. Daraus kann gefolgert werden, dass beide in der Kopfgruppe des Lysinollipids vorhandenen Aminogruppen (vgl. Abbildung 5.6) für Kieselsäure zugänglich und an der Reaktion beteiligt sind und dadurch die Masse erhöht wird. In dem Fall wird vermutlich nicht nur eine Silicamonoschicht an der Vesikeloberfläche gebildet.

Die Erkenntnisse lassen sich wie folgt zusammenfassen:

- Hohe Ionenstärken verringern die Präzipitationsmenge, da positive Ladungen abgeschirmt werden.

- Je mehr Moleküle in vesikulärer Form und nicht in mizellarer Form aggregiert sind, desto mehr Silica kann gebildet werden, woraus gefolgert wird, dass Mizellen ein geringeres Präzipitationspotenzial haben.

- Je höher die Ladungsdichte auf der Vesikeloberfläche, umso größer ist die präzipitierte Silicamenge. 
Die Lichtstreuungsexperimente lieferten Ergebnisse, die mit den oben erläuterten Trends übereinstimmen. Auch wenn sich hier die Beobachtungen nicht auf die Silicamengen, sondern auf die Zunahme der hydrodynamischen Radien der Aggregate (Abbildung 5.3 B) beziehen, festigen die Ergebnisse die aufgestellten Hypothesen. Weiterhin konnte der zeitliche Verlauf der Reaktion von Kieselsäure mit Vesikeln der unterschiedlichen Aminolipide verfolgt werden. Hier zeigte sich, dass diese sehr schnell ablaufen, bereits nach ca. acht Minuten konnte bei allen untersuchten Systemen keine wesentliche Änderung des hydrodynamischen Radius mehr detektiert werden. Die Bildung der Silicazellwände von Diatomeen dauert oftmals nicht länger als ein bis zwei Stunden. ${ }^{161}$ Das deutet darauf hin, dass auch in den Organismen schnelle Reaktionen ablaufen müssen, damit die vollständige Struktur innerhalb dieser Zeit gebildet werden kann. Die kurze Dauer ist einer der Vorteile, die die biomimetische Reaktion gegenüber technischen Silicapolymerisationsverfahren hat, welche unter drastischen Reaktionsbedingungen teilweise bis $\mathrm{zu}$ mehrere Tage dauern. ${ }^{35,38,162}$

In Kapitel 5.3 wurden elektronenmikroskopische Aufnahmen der Silicapräzipitate gezeigt (Abbildungen 5.4 und 5.5). In Kombination mit den Daten, die mit dem $\beta$-Molybdattest beziehungsweise der Lichtstreuung gewonnen wurden, belegen die Aufnahmen die Bildung von Silica, ausgelöst durch in Vesikel aggregierte Aminolipide.

Ein Vergleich der Präzipitate, bei denen nach der Bildung durch Waschen und Zentrifugation überschüssige Kieselsäure entfernt wurde (Abbildung 5.5), mit denen, bei denen diese Behandlung nicht möglich war (Abbildung 5.4), zeigt deutliche Unterschiede. Denn während die mittels $\beta$-Molybdattest detektierten Siliciummassen darauf hindeuten, dass sich um die Vesikel lediglich eine oder zwei atomare Silicaschichten bilden (vgl. Tabelle 5.3), lassen die Lichtstreuungsdaten auf größere Aggregate im Mikrometerbereich schließen (Abbildung 5.3). Die SEM-Aufnahmen bestätigen diese Ergebnisse. Die Größe der Silicastrukturen der Präzipitate, die bei den Lichtstreuungsmessungen entstanden und nicht weiter behandelt wurden, sind sehr groß und weisen Durchmesser im Mikrometerbereich auf (Abbildung 5.4). Dahingegen treten bei den Proben, die wie diejenigen beim $\beta$-Molybdattest behandelt wurden, Strukturen auf, die in derselben Größenordnung wie die Vesikel liegen. Zudem sind teilweise kollabierte Kugeln erkennbar, was darauf schließen lässt, dass die Silicaschichten tatsächlich sehr dünn sind, worauf die oben erläuterten Erkenntnisse schließen lassen. Die unterschiedliche 
Behandlung der Proben hat demnach einen wesentlichen Einfluss auf die Struktur der Silicapartikel. Des Weiteren kann festgestellt werden, dass die Verwendung der Vesikel als strukturvorgebende Template teilweise erfolgreich war. In einigen Proben wurden intakte Kugeln im Größenbereich der Vesikel beobachtet (Abbildung 5.5 C). Sie ähneln hohlen Silicasphären die in anderen Untersuchungen beschrieben wurden, wo ebenfalls kationische Vesikel als Template für die Silicabildung eingesetzt wurden, jedoch nur teilweise unter biomimetischen Bedingungen. ${ }^{163,164}$ In diesen Arbeiten wurden die Schichtdicken dieser Sphären zu 1-2 $\mathrm{nm}^{164}$ beziehungsweise ca. $10 \mathrm{~nm}^{163}$ bestimmt was insofern mit den Erkenntnissen dieser Arbeit in Einklang steht, dass auch hier die Schichtdicken sehr gering sind (Abbildung 5.5).

Zusammenfassend lässt sich aussagen, dass an der Oberfläche von Vesikeln der Aminolipide Silicapräzipitation stattfindet und ihre Struktur dabei teilweise abgebildet wird. Die Reaktionen finden auf einer schnellen Zeitskala und unter physiologischen Bedingungen statt. Der Einsatz der Vesikel als Aggregate erlaubte die Untersuchung des Einflusses einzelner Faktoren auf die Reaktion. Sind alle Moleküle in Vesikeln angeordnet, so sind die entscheidenden Parameter für die Reaktivität die Protonierung der Aminogruppen sowie die Anzahl anwesender Aminoeinheiten.

Die unterschiedlichen $\mathrm{pK}_{\mathrm{s}}$-Werte und daraus resultierenden Ladungsdichten sind durch die unterschiedlich starke $N$-Methylierung bedingt, die auch in Silaffinen und Polyaminen der Diatomeen variiert. Die in dieser Arbeit belegte Abhängigkeit der Reaktivität von der Alkylierung der Aminogruppen trifft wahrscheinlich auf für die in den Organismen vorkommenden Peptide und Polyamine zu, und könnte zusammen mit anderen Faktoren bei der Bildung der hierarchischen speziesspezifischen Zellwände eine Rolle spielen. 



\section{Aminolipide in planaren Membranen}

Die Funktionalität der in Kapitel 5 vorgestellten Aminolipide bezüglich der biomimetischen Bildung von Silica konnte nachgewiesen werden. Sind die amphiphilen, lipidähnlichen Moleküle in vesikulären Strukturen arrangiert, induzieren sie in Lösung innerhalb weniger Minuten Präzipitationsreaktionen. Hierbei zeigten sich Unterschiede im Präzipitationspotential in Abhängigkeit der Aminokopfgruppe. Als besonders effektiv erwies sich dasjenige Aminolipid, das eine Lysinoleinheit mit zwei primären Aminoeinheiten aufweist. Davon ausgehend stellte sich die Frage, ob auch an der Oberfläche von planar angeordneten Aminolipidschichten $\mathrm{SiO}_{2}$-Bildung ausgelöst werden kann und auf diese Art und Weise flache Silicafilme hergestellt werden können. Die Untersuchungen konzentrierten sich hierbei auf das Lysinollipid, zu welchem Informationen Tabellen 5.1, 5.2 sowie Abbildung 5.6 entnommen werden können. Zunächst galt es, Doppelschichten des Lipids aufzubauen und $\mathrm{zu}$ charakterisieren. Im Anschluss daran, sollte die Silicabildung an der Oberfläche der Membran analysiert werden. Mit Hilfe eines weiteren Konzepts sollte der Aufbau planarer Silicaschichten mit Poren definierter Größe realisiert werden. Dazu wurde neben dem Lysinollipid ein Glykolipid, C16-Glycerol- $\beta$-Lactosid (C16-GL), eingesetzt, welches keine Silica-präzipitierende Funktion besitzen und aufgrund seiner Struktur in Membrandomänen angereichert werden sollte. Zunächst mussten diese Hypothesen geprüft werden, bevor auch an der Oberfläche dieses gemischten Systems die Silicapräzipitation genauer untersucht werden konnte.

\subsection{Lysinollipid}

\subsubsection{Charakterisierung der festkörperunterstützten Doppelschichten}

Wie in Kapitel 5.2 gezeigt werden konnte, lagert sich das Lysinollipid aufgrund seines amphiphilen Charakters in lamellaren Strukturen zu Vesikeln zusammen. In der Regel können Substanzen mit dieser Eigenschaft auch planare Doppelschichten bilden. Gängige Präparationsmethoden sind unter anderem das Spreiten von Vesikeln sowie LangmuirBlodgett-(LB)-Überträge auf hydrophilen Oberflächen wie Siliciumwafern, Glas oder Glimmer. Die zwei Methoden bieten unterschiedliche Vorteile, die später näher erläutert werden, weshalb mit beiden Lysinollipiddoppelschichten aufgebaut wurden. Als Substrate 
wurden jeweils Si-Wafer mit hydrophilen $\mathrm{SiO}_{2}$-Schichten verwendet. Die Dicke der $\mathrm{SiO}_{2}-$ Schichten richtet sich nach dem Verwendungszweck. Da Silicium Fluoreszenz löscht, werden oftmals für fluoreszenzmikroskopische Untersuchungen Wafer mit dickeren $\mathrm{SiO}_{2}$ Schichten (20-100) nm gewählt, um höhere Intensitäten zu erhalten, als dies auf (1-10) nm dicken nativen Oxidschichten der Fall ist.

\section{Spreiten von Vesikeln}

Zum Spreiten wurden, wie in Kapitel 3.2.1 beschrieben, Vesikel eines nominellen Durchmessers von $100 \mathrm{~nm}$ in $25 \mathrm{mM}$ MES/500 mM KCl pH 6,0 hergestellt. Der Prozess der Membranbildung auf der Siliciumoberfläche wurde zunächst mittels Ellipsometriemessungen verfolgt. Hierzu wurden definierte Volumina von Lysinollipidvesikelsuspensionen ( $c=1,6 \mathrm{mM})$ zu einer Pufferlösung (50 mM MES pH 6,0) über dem Substrat zugegeben (resultierende Lipidkonzentration: $c=0,2 \mathrm{mM}$ ) und die Veränderungen auf der Waferoberfläche durch Messung der ellipsometrischen Winkel Delta und Psi beobachtet (Abbildung 6.1). Vor Zugabe der Vesikel sind die Werte beider Winkel konstant. Ab dem Zeitpunkt der Zugabe ( $t=2$ min) steigt Psi an, während Delta schnell kleiner wird.

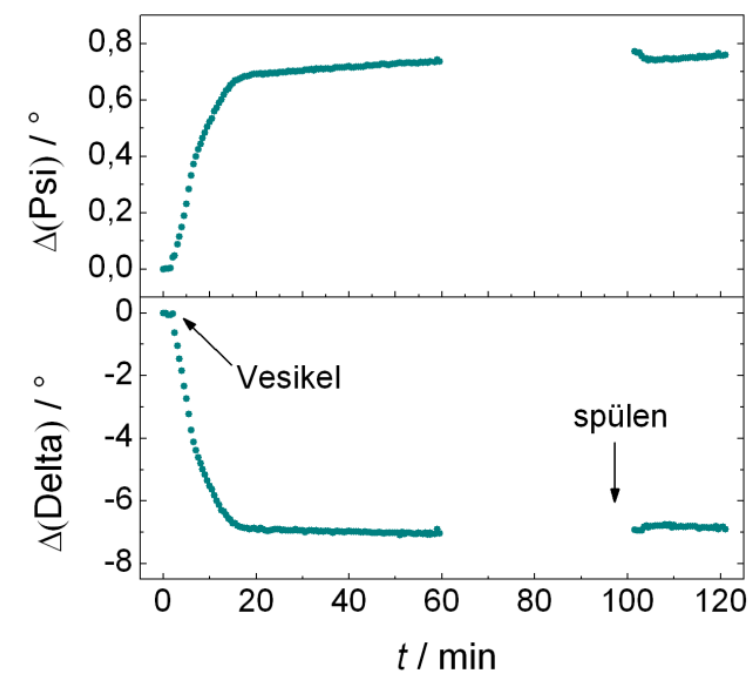

Abbildung 6.1 Zeitliche Änderung der ellipsometrischen Winkel Delta und Psi nach Zugabe von Lysinollipidvesikeln $(d=100 \mathrm{~nm})$ bei $t=2 \mathrm{~min} \mathrm{zu}$ einem Substrat (Si-Wafer mit nativer $\left.\mathrm{SiO}_{2}-\mathrm{Schich}\right)$. Messpuffer: $50 \mathrm{mM}$ MES pH 6,0; $c_{\text {Lipid }}=0,2 \mathrm{mM}$.

Ab ca. fünfzehn Minuten nach der Zugabe werden keine signifikanten Änderungen mehr detektiert und auch nach Spülen der Messzelle mit Puffer sind die Werte weitestgehend unverändert. Obwohl die Messung keine Schichtdicken liefert, können qualitative Aussagen getroffen werden. Die Abnahme des Winkels Delta bei gleichzeitiger Erhöhung 
von Psi entspricht bei den verwendeten Messbedingungen der Zunahme einer Schichtdicke auf dem Wafer. Es kann davon ausgegangen werden, dass diese Änderung durch die Anlagerung und eventuell das Spreiten von Vesikeln auf der Oberfläche bedingt wurde.

Um zu klären, ob diese Vesikel gespreitet haben und sich eine gleichmäßige Lipiddoppelschicht ausgebildet hat, wurden die Proben anschließend mikroskopisch untersucht. $\mathrm{Zu}$ diesem Zweck wurden jeweils $0,5 \mathrm{~mol} \%$ eines fluoreszenzmarkierten Lipids (Sulforhodamin 101 DHPE) zugesetzt, das sich in den lamellaren Aggregaten verteilt, und eine fluoreszenzmikroskopische Charakterisierung ermöglicht. In den entsprechenden Aufnahmen (Abbildung 6.2) sind regelmäßig leuchtende Oberflächen erkennbar, woraus geschlossen werden kann, dass die Vesikel gespreitet haben und Lipidschichten auf den Substraten vorlagen. Vereinzelt treten dunkle Bereiche auf (Abbildung 6.2 B), die durch Defekte in den Membranen verursacht werden. In Aufnahme C, die am Rand eines Substrates (rechts oben) entstand, zeigen sich stellenweise helle, an die Oberfläche gebundene Vesikel.
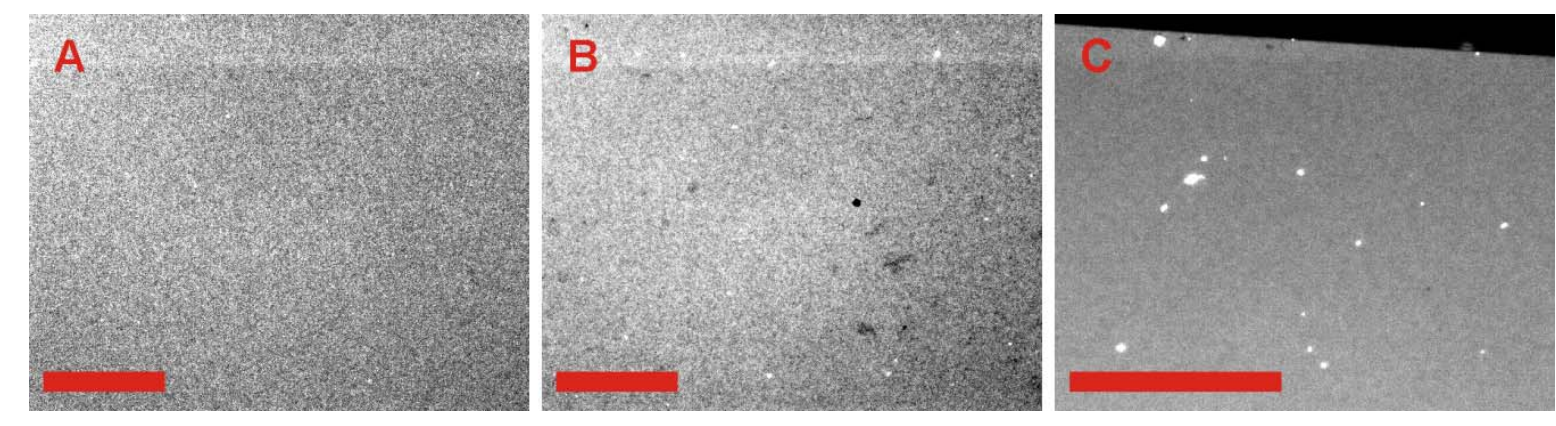

Abbildung 6.2 Fluoreszenzmikroskopische Aufnahmen von Proben, die durch einstündige Inkubation von Lysinollipidvesikeln $\left(d=100 \mathrm{~nm}, c_{\text {Lipid }}: 0,2 \mathrm{mM}, 0,5 \mathrm{~mol} \%\right.$ Sulforhodamin 101 DHPE) in $50 \mathrm{mM}$ MES pH 6,0 auf Si-Wafern (100 nm Oxid) entstanden. Maßstabsbalken: $50 \mu \mathrm{m}$.

Um die Proben mit höherer Auflösung charakterisieren und Aussagen über ihre Textur treffen zu können, wurden rasterkraftmikroskopische (AFM) Messungen durchgeführt. Wie in Abbildung 6.3 erkennbar ist, sind die Oberflächen eben und besitzen mit ca. $1 \mathrm{~nm}$ hohen Stufen im Vergleich zu Phospholipiddoppelschichten eine vergleichsweise hohe Rauheit (Abbildung 6.3 C). ${ }^{165-167}$ Diese wurde für Aufnahme B mit der Software SPIP zu $S_{a}=0,2 \mathrm{~nm}$ bestimmt, wobei $S_{a}$ das arithmetische Mittel der 3D-Rauheit bezeichnet. Da bekannt ist, dass Vesikel positiv geladener Lipide auf hydrophilen Oberflächen Doppelschichten ausbilden, ${ }^{168,169}$ kann in Übereinstimmung mit den Daten aus den ellipsometrischen und fluoreszenzmikroskopischen Messungen gefolgert werden, dass das Lysinollipid auf der hydrophilen Oberfläche in regelmäßigen, weitestgehend defektfreien Doppelschichten angeordnet vorliegt. 

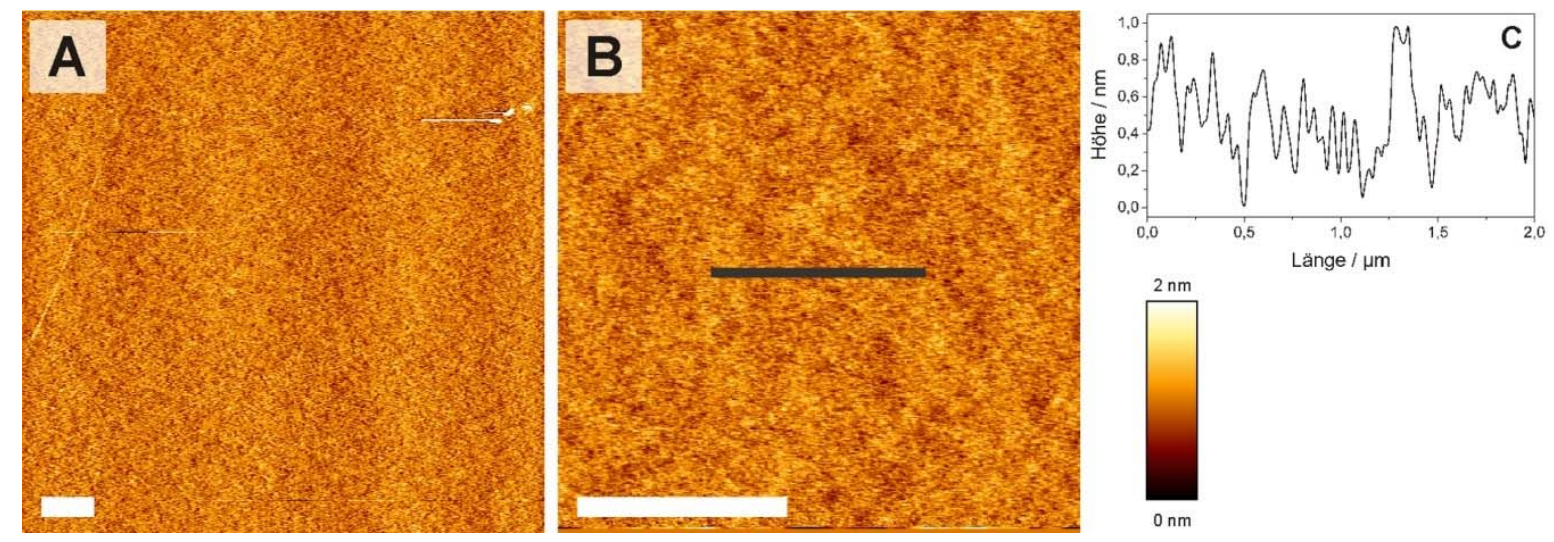

Abbildung 6.3 A/B Topografische AFM-Aufnahmen von auf Si-Wafern (100 nm Oxid) gespreiteten Lysinollipidvesikeln $\left(d=100 \mathrm{~nm}, c_{\text {Lipid }}\right.$ : 0,2 mM). Die Messung wurde in Reinstwasser im tapping mode (NWII) mit MSCT-Au-cantilevern durchgeführt. Maßstabsbalken: $2 \mu \mathrm{m}$. C Höhenprofil aus Aufnahme B (schwarze Linie).

\section{Langmuir-Blodgett-Überträge}

Lipidschichten können von der Luft-Wasser-Grenzfläche mittels Langmuir-Blodgett (LB)Technik auf Substrate immobilisiert werden. Hierbei wird jeweils eine Monoschicht übertragen, weshalb für die Bildung einer Doppelschicht die Prozedur wiederholt werden muss. Die Kopfgruppen der Lipide der unteren Monoschicht sind zum hydrophilen Substrat hin orientiert und beeinflussen somit die Prozesse und Reaktionen bei der Präzipitation nicht, die später an der Oberfläche der Doppelschicht stattfinden sollen. Die untere Monoschicht muss jedoch eine möglichst dichte und ebene hydrophobe Oberfläche bilden, damit die zweite Monoschicht darauf immobilisiert werden kann. In dieser Arbeit wurde eine Monoschicht aus 1,2-Dipalmitoyl-sn-glycero-3-phosphatidylcholin (DPPC) gewählt, dessen Verhalten an der Luft-Wasser-Grenzfläche gut untersucht ist und das bei hohen Oberflächendrücken in der $l_{\mathrm{c}}$-Phase vorliegt. Die Monoschicht wurde auf einer Filmwaage bis zu einem Oberflächendruck von $45 \mathrm{mN} / \mathrm{m}$ komprimiert, wobei eine für DPPC typische Isotherme ${ }^{170,171}$ beobachtet wurde (Abbildung $6.4 \mathrm{~A}$ ). Der Oberflächendruck des Films wurde anschließend konstant bei $45 \mathrm{mN} / \mathrm{m}$ gehalten während ein Si-Wafer aus der Subphase herausgezogen wurde. Durch die Übertragung von Molekülen auf das Substrat sinkt der Oberflächendruck. Dadurch ausgelöst reguliert die Filmwaage nach, indem die Filmfläche verkleinert wird bis der Sollwert des Oberflächenwerts wieder erreicht ist. Die kontinuierliche Verringerung der Filmfläche gilt als Nachweis für die erfolgreiche Immobilisierung einer Monoschicht (Abbildung 6.4 B). Auf die so hergestellten beschichteten Substrate mit hydrophober Oberfläche wurden im nächsten Schritt Lysinollipidmonoschichten übertragen, die zuvor auf Wasser zu einem 
Oberflächendruck von $27 \mathrm{mN} / \mathrm{m}$ komprimiert wurden. Der Kurvenverlauf der Isotherme für das Lysinollipid ist in Abbildung 6.4 C dargestellt. Während der DPPC/Si-Wafer in die Subphase eingetaucht wurde, verkleinerte sich wiederum die Filmfläche, was für eine erfolgreiche Immobilisierung der Lysinollipidschicht spricht (Abbildung 6.4 D).
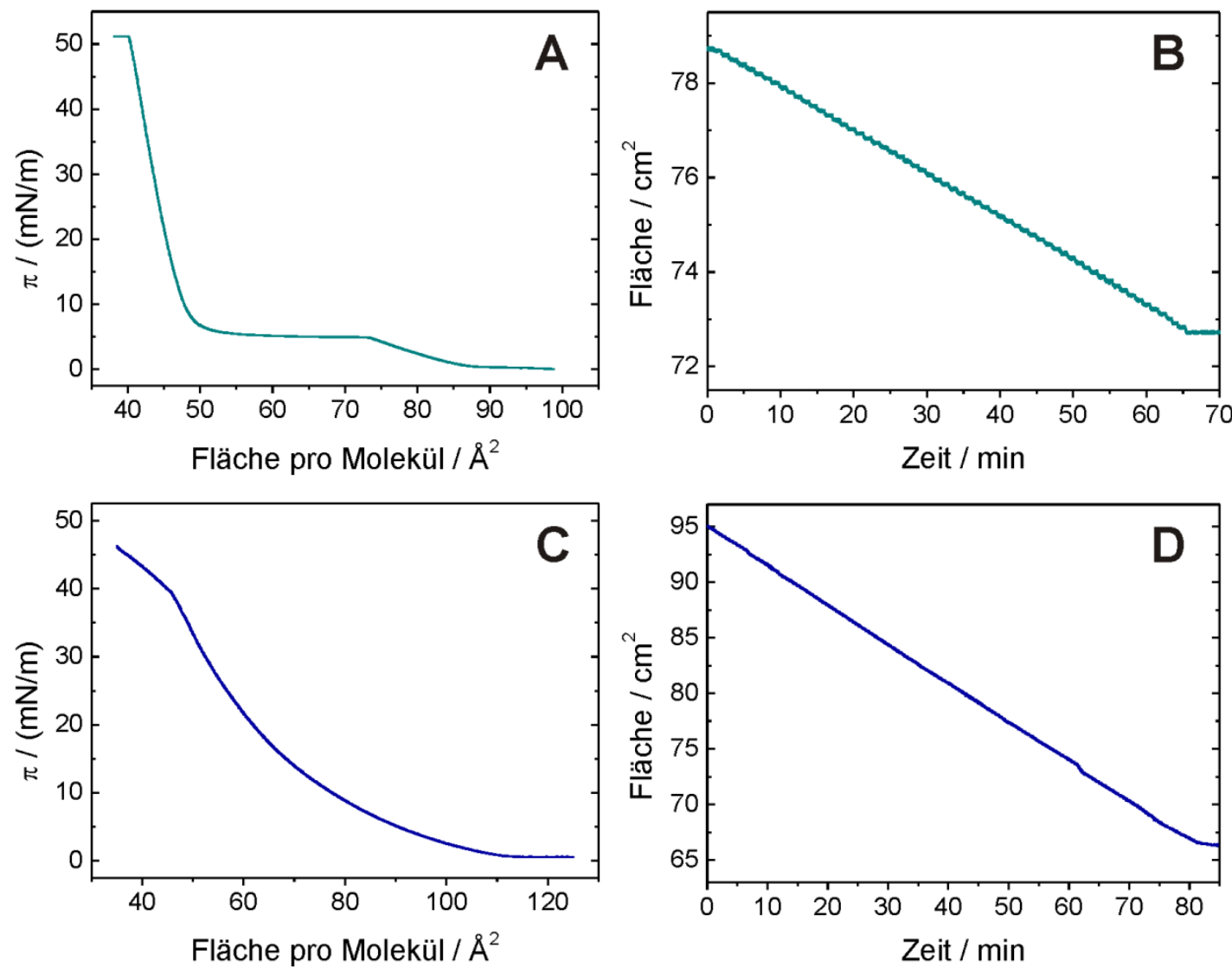

Abbildung 6.4 $\pi / A$-Isothermen für A DPPC und C Lysinollipid gemessen auf Reinstwasser bei $20^{\circ} \mathrm{C}$. Zeitliche Änderung der Gesamtfilmfläche während des LB-Übertrags von B DPPC- beziehungsweise D Lysinollipidmonoschichten auf Siliciumwafer $\left(100 \mathrm{~nm} \mathrm{SiO}_{2}\right)$.

Die auf diese Art hergestellten Lipidschichten wurden fluoreszenzmikroskopisch untersucht (Abbildung 6.5). In Aufnahmen A-C, in denen nur der Lysinollipidschicht, also der oberen Monoschicht, ein Fluoreszenzfarbstoff beigefügt wurde, ist erkennbar, dass Lipid immobilisiert wurde, die Oberfläche aber heterogen ist. Es treten sehr häufig dunkle Streifen senkrecht zur Aufziehrichtung des Übertrages auf (B), teilweise auch parallel dazu (A) und es gibt runde dunkle Flecken und diffus verlaufende Streifen (C). Wird auch die untere DPPC-Monoschicht fluoreszenzmarkiert (D), sind die zuvor beschriebenen Charakteristika wie Streifen und runde Flecken ebenfalls erkennbar, jedoch ist das Aussehen durch Fluoreszenz-Resonanzenergietransfer-Effekte (Fluorescence resonance energy transfer, FRET) verändert, worauf im nächsten Abschnitt näher eingegangen wird. 


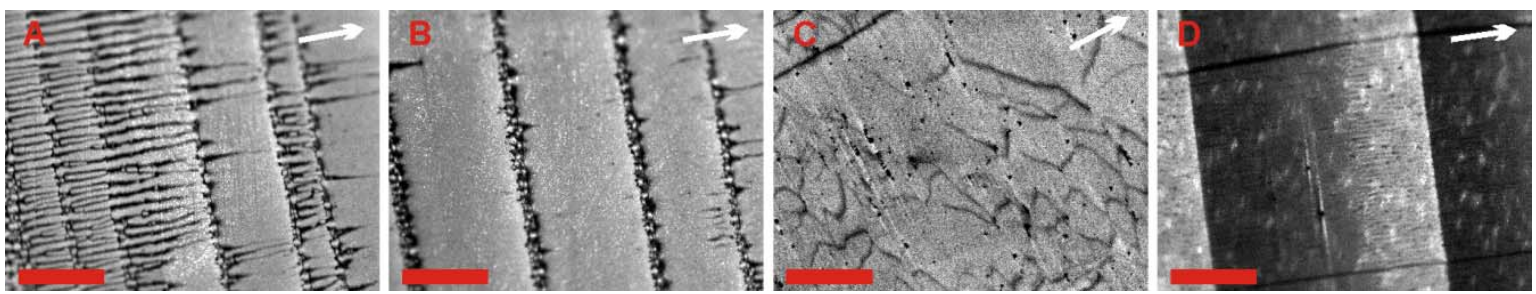

Abbildung 6.5 Fluoreszenzmikroskopische Bilder einer Lysinollipidmonoschicht, versetzt mit A-C 0,5 mol\% oder D 0,2 mol\% Sulforhodamin 101 DHPE, mit LB-Technik bei $\pi=27 \mathrm{mN} / \mathrm{m}$ übertragen auf DPPC/Si-Wafer. Die DPPC-Monoschicht wurde bei $\pi=45 \mathrm{mN} / \mathrm{m}$ auf Si-Wafer (100 nm Oxid) immobilisiert und enthielt A-C kein beziehungsweise D 0,5 mol\% $\beta$-BODIPY 500/510 $\mathrm{C}_{12}$-HPC. Der weiße Pfeil markiert die jeweilige Aufziehrichtung beim LB-Übertrag. Maßstabsbalken: $50 \mu \mathrm{m}$.

Anhand der fluoreszenzmikroskopischen Abbildungen kann nicht eindeutig geklärt werden, ob es sich bei den dunklen Bereichen um Defekte oder Membranstrukturen handelt, weshalb auch diese Oberflächen rasterkraftmikroskopisch untersucht wurden. Hierbei zeigte sich, dass sich die zuvor beschriebenen Merkmale auch in den topografischen Aufnahmen wiederfinden (Abbildung 6.6).
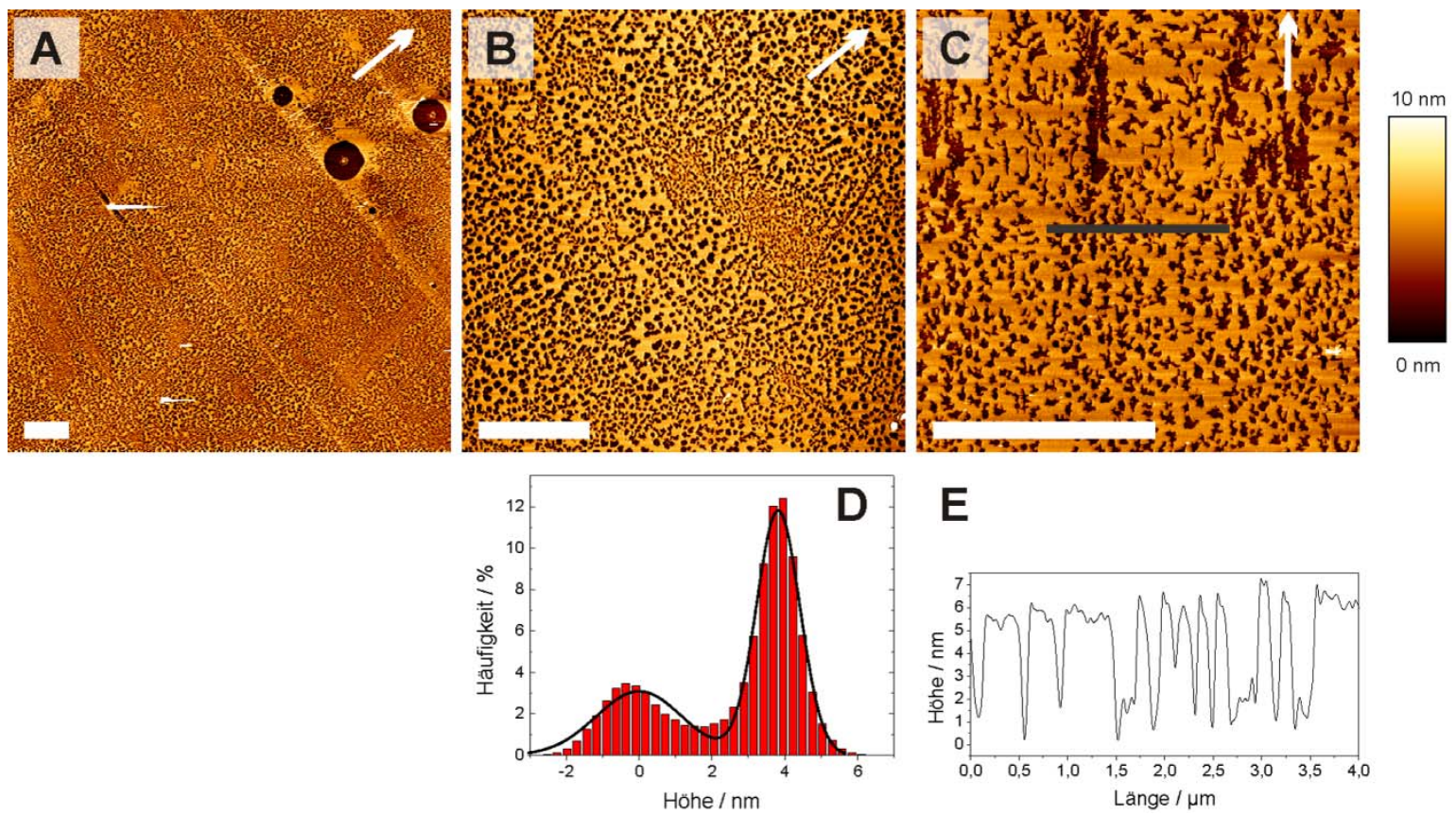

Abbildung 6.6 A-C Rasterkraftmikroskopische Aufnahmen von Lysinollipidmonoschichten immobilisiert auf DPPC/Si-Wafer (native Oxidschicht) bei $\pi=25-27 \mathrm{mN} / \mathrm{m}$. Die Messungen wurden im tapping mode (NWI) mit CSC37-cantilevern in Reinstwasser durchgeführt. Maßstabsbalken: $5 \mu \mathrm{m}$. Die Pfeile zeigen die Aufziehrichtung des LB-Übertrags an. D Histogrammanalyse der Höhenverteilung in Aufnahme C. E Höhenprofil entlang der schwarzen Linie in Aufnahme C.

Quer zur Aufziehrichtung sind Streifen erkennbar und die runden Objekte können als Löcher mit Durchmessern von ca. $5 \mu \mathrm{m}$ identifiziert werden (A). Es konnten zwei Höhenniveaus beobachtet werden, deren Differenz durch Höhenanalysen (D,E) zu $(4,2 \pm 1,6) \mathrm{nm}(n=4)$ bestimmt wurde, was der erwarteten Höhe einer Doppelschicht 
entspricht. Dementsprechend können die höheren Bereiche der Doppelschicht und die tieferen Membrandefekten zugeordnet werden und es kann gefolgert werden, dass es sich bei den dunklen Stellen in den Fluoreszenzaufnahmen (Abbildung 6.5) um Defekte handelt. Die Rauheit wurde exemplarisch für Aufnahme $\mathrm{C}$ bestimmt und beträgt $S_{a}=1,6 \mathrm{~nm}$. Sie ist aufgrund der vielen Defekte höher als bei den gespreiteten Doppelschichten.

Es lässt sich zusammenfassen, dass die Immobilisierung von Lysinollipidmonoschichten durch Langmuir-Blodgett-Technik prinzipiell funktioniert, jedoch keine defektfreien Membranen erzeugt werden können, wie dies durch Spreiten von Vesikeln erreicht wurde.

\subsubsection{Präzipitationsreaktionen an planaren Lysinollipiddoppelschichten}

Die im vorhergehenden Kapitel vorgestellten Doppelschichten sind an ihrer Oberfläche aufgrund der sich dort befindenden Aminogruppen der Kopfgruppe des Lysinollipids positiv geladen und sollten theoretisch die Präzipitation von Kieselsäure auslösen. Analog zu den Präzipitationsexperimenten in Lösung wurde $\mathrm{Si}(\mathrm{OH})_{4}$ als Monomer eingesetzt, das durch Hydrolyse von Tetramethoxysilan in $\mathrm{HCl}$ erhalten wurde. Die Monokieselsäure wurde jeweils zum Puffer (50 mM MES pH 6,0), mit dem das Substrat mit der darauf immobilisierten Lysinollipidmembran überschichtet war, gegeben und die Silicabildung mit verschiedenen Messmethoden beobachtet und charakterisiert.

\section{Ellipsometrische Untersuchung des Silicabildungsprozesses}

Zunächst wurden ellipsometrische Messungen durchgeführt. Die Silicabildung wurde sowohl an gespreiteten (Abbildung 6.7 A) als auch an mittels LB-Übertrag (Abbildung 6.7 B) präparierten Schichten untersucht. Vor Zugabe von $\mathrm{Si}(\mathrm{OH})_{4}$ zu einer gespreiteten Membran (A) sind die beiden ellipsometrischen Winkel Delta und Psi konstant, ab dem Zeitpunkt der Zugabe ( $t=2 \mathrm{~min})$ treten deutliche Änderungen auf. Der Winkel Delta wird rapide kleiner, acht Minuten nach der Zugabe $(t=10 \mathrm{~min})$ beträgt die Änderung $-3^{\circ}$. Die Steigung der Kurve nimmt anschließend ab, zum Zeitpunkt $t=25 \mathrm{~min}$ beträgt die Differenz zum Startwert $-3,3^{\circ}$ und nach sechzig Minuten $-3,6^{\circ}$. Anschließend wurden die Membran mit MES-Puffer gespült, um überschüssige Kieselsäure zu entfernen, wonach sich Delta nicht mehr signifikant änderte und um ca. 3,9 ${ }^{\circ}$ kleiner ist als zu Beginn der 
Messung. Der zeitliche Verlauf der Kurve für den Winkel Psi gleicht dem für Delta, mit dem Unterschied, dass Psi ansteigt. Nach dem Spülen mit Puffer ist der Wert von Psi um ca. $0,7^{\circ}$ höher als vor der Kieselsäurezugabe. Diese Veränderungen der ellipsometrischen Winkel bedeuten für das vorliegende System eine Zunahme der Schichtdicke und sind somit ein Hinweis für die Ablagerung von Silica auf der Oberfläche. Auch bei Verwendung von Doppelschichten, die mittels LB-Technik herstellt wurden verringerte sich Delta, während Psi größer wurde (Abbildung $6.7 \mathrm{~B}$ ). Beide Winkel sind bis zur Zugabe von $\mathrm{Si}(\mathrm{OH})_{4}(t=5 \mathrm{~min})$ weitestgehend konstant. Innerhalb von fünf Minuten fällt Delta um $0,8^{\circ}$, woraufhin die Kurve flacher wird. Zum Zeitpunkt $t=25$ min beträgt die Änderung $-1,4^{\circ}$, nach sechzig Minuten $-1,6^{\circ}$. Nach dem Spülen der Membran pendelt sich $\Delta$ (Delta) bei ca. $-1,7^{\circ}$ ein. Psi steigt im Verlauf der gesamten Messung um 0,3 ${ }^{\circ}$. Auch auf Lysinol-LB-Schichten lagert sich demnach $\mathrm{SiO}_{2}$ ab. Es kann an dieser Stelle lediglich eine qualitative Aussage getroffen werden, da die quantitativen Veränderungen der Winkel nicht miteinander verglichen werden können. Bei beiden Systemen liegt jedoch eine Zunahme der Schichtdicke auf dem Substrat vor, die in einem schnell Prozess verläuft und nach nur $(10-20)$ min beendet ist.
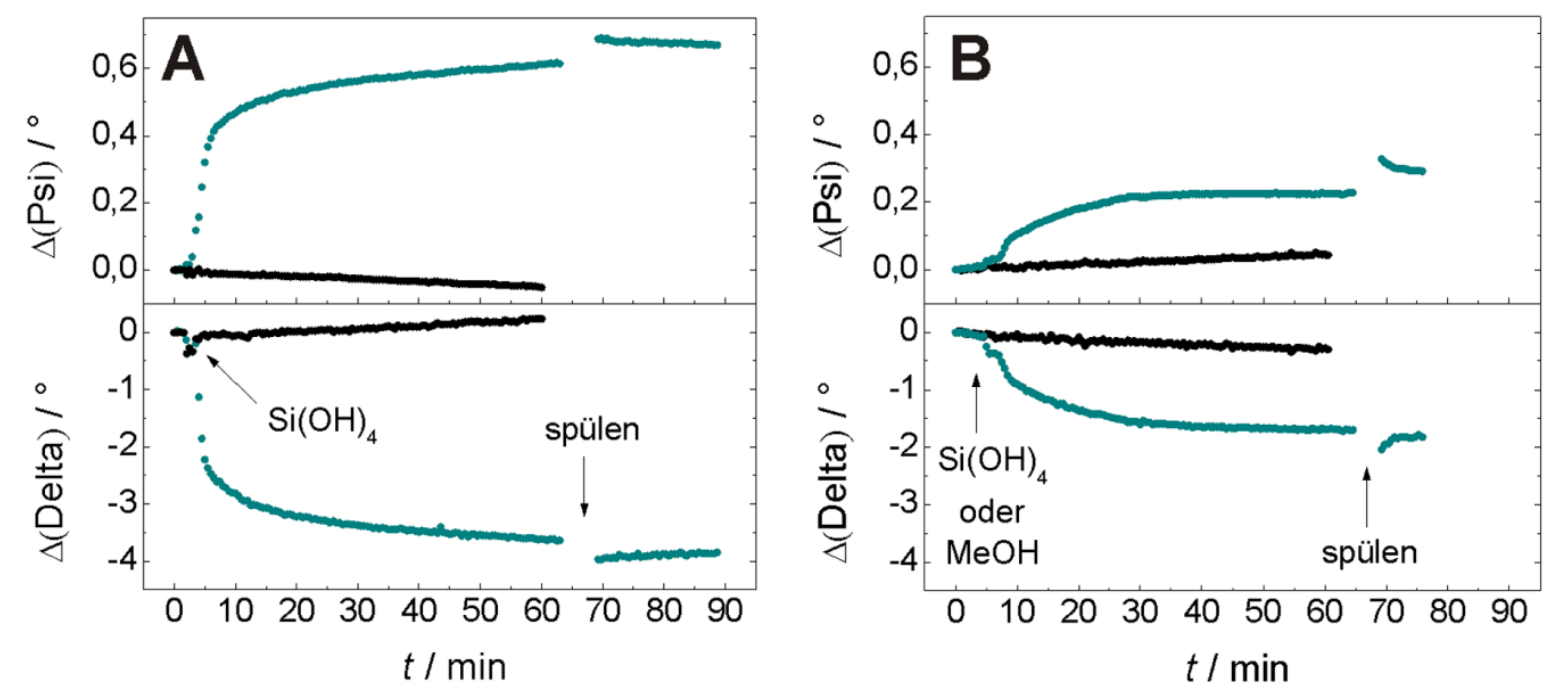

Abbildung 6.7 Ellipsometriemessungen: • Zugabe von $50 \mathrm{mM} \mathrm{Si}(\mathrm{OH})_{4}$ zu Lysinollipidschichten, die mittels A Vesikelspreiten und B LB-Technik präpariert wurden und anschließendes Spülen mit Puffer. - Kontrollexperimente: A Zugabe von $\sim 190 \mathrm{mM} \mathrm{Si}(\mathrm{OH})_{4}$ zu einem Si-Wafer. B Zugabe von $\sim 730 \mathrm{mM}$ Methanol in $1 \mathrm{mM} \mathrm{HCl} \mathrm{zu} \mathrm{einer} \mathrm{Lysinol-LB-Doppelschicht.} \mathrm{Si-Wafer:} \mathrm{jeweils} \mathrm{mit} \mathrm{nativer} \mathrm{Oxidschicht.} \mathrm{Alle}$ Messungen wurden in $50 \mathrm{mM}$ MES-Puffer $\mathrm{pH}$ 6,0 durchgeführt.

Um sichergehen zu können, dass die gemessenen Effekte durch eine spezifische Interaktion der Lysinollipide mit Monokieselsäure ausgelöst wurden, wurden zwei Kontrollexperimente durchgeführt. Zum einen wurde $\mathrm{Si}(\mathrm{OH})_{4} \mathrm{zu}$ einem Siliciumwafer 
ohne immobilisierte Membran gegeben (Abbildung $6.7 \mathrm{~A}$ ), zum anderen wurde eine Lysinollipidschicht mit Methanol und $\mathrm{HCl}$ versetzt, da diese Substanzen auch in der $\mathrm{Si}(\mathrm{OH})_{4}$-Lösung vorhanden sind (Abbildung 6.7 B). In beiden Fällen wurden über den gemessenen Zeitbereich keine signifikanten Änderungen von Delta und Psi beobachtet.

Um weitere Erkenntnisse über die Vorgänge an der Oberfläche der Membranen gewinnen zu können, wurden die Proben sowohl fluoreszenz- also auch rasterkraftmikroskopisch untersucht. Auch hier wurden, wie oben beschrieben, unterschiedlich hergestellte Membranen eingesetzt.

\section{Silicastrukturen an Lysinollipidschichten (Vesikelspreiten)}

Die Fluoreszenzaufnahmen von Doppelschichten, die durch Spreiten von Vesikeln präpariert und im Anschluss mit Monokieselsäure inkubiert wurden (Abbildung $6.8 \mathrm{~A} / \mathrm{B}$ ), geben das gleiche Bild wie vor der $\mathrm{Si}(\mathrm{OH})_{4}$-Zugabe wieder (Abbildung 6.2). Teilweise sieht die Oberfläche inhomogen aus (Abbildung $6.8 \mathrm{C}$ ) und wie auch in den anderen Bildern sind vereinzelt hell leuchtende Stellen zu beobachten. Die Untersuchung zeigt, dass die Membranen bei Inkubation mit Kieselsäure nicht zerstört werden.
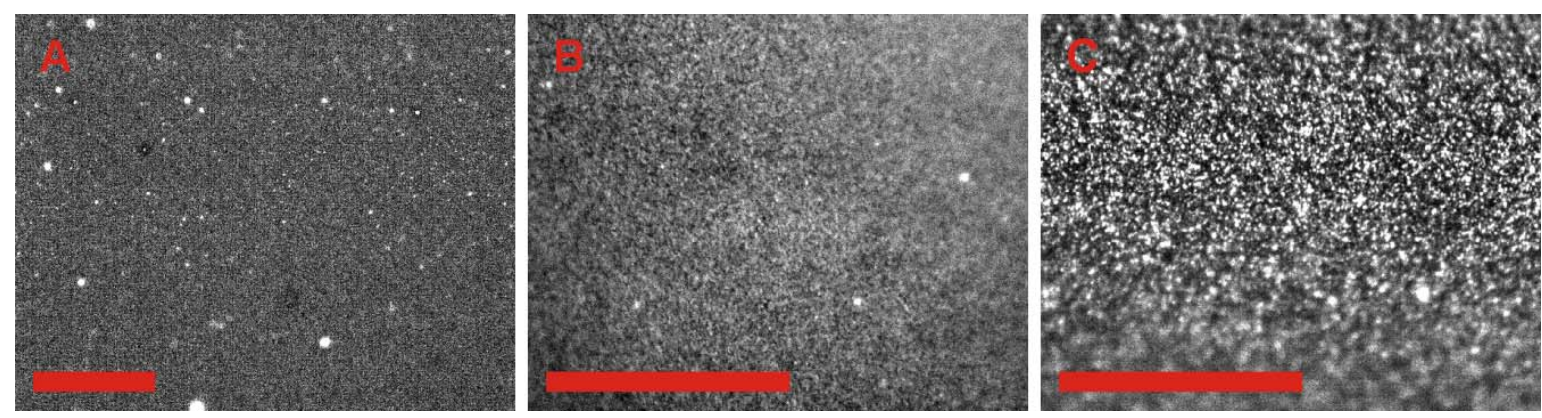

Abbildung 6.8 Fluoreszenzaufnahmen von Lysinoldoppelschichten (0,5 mol\% Sulforhodamin 101 DHPE), die auf Si-Wafern mit A/C nativer beziehungsweise $\mathbf{B} 100 \mathrm{~nm} \mathrm{SiO}$-Schicht aus Vesikeln gespreitet wurden und $60 \mathrm{~min}$ mit $50 \mathrm{~mm} \mathrm{Si}(\mathrm{OH})_{4}$ in $50 \mathrm{~mm}$ MES-Puffer pH 6,0 inkubiert und anschließend mit Puffer gespült wurden. Maßstabsbalken: $50 \mu \mathrm{m}$.

Um eventuell auftretende Veränderungen durch Interaktion mit $\mathrm{Si}(\mathrm{OH})_{4}$ erkennen und näher spezifizieren zu können, wurden die Proben im Anschluss rasterkraftmikroskopisch untersucht. Die topografischen AFM-Aufnahmen verdeutlichen, dass sich die Oberflächen durch Zugabe der monomeren Kieselsäure verändert haben (Abbildung 6.9). Während sie zuvor glatt erschienen (Abbildung 6.3), wirken sie nun inhomogen und weniger eben, was auch das Höhenprofil (C) bestätigt. Es sind runde Objekte mit einer Höhe von bis zu $6 \mathrm{~nm}$ erkennbar, die Durchmesser von ca. (50-100) nm aufweisen. Die Rauheit hat zugenommen 
und beträgt nun $S_{a}=0,8 \mathrm{~nm}$ (Abbildung $6.9 \mathrm{~B}$ ) im Gegensatz zu $0,2 \mathrm{~nm}$ vor der $\mathrm{Si}(\mathrm{OH})_{4^{-}}$ Zugabe.

In Kombination mit der Schichtdickenzunahme, die bei den ellipsometrischen Untersuchungen ermittelt wurde, sprechen die AFM-Messungen für die Ablagerung von Silica auf der Oberfläche der Lysinollipidschicht. Da es weder durch Spülen mit Puffer noch durch das Abtasten mit der cantilever-Spitze abgetragen wird, kann davon ausgegangen werden, dass es irreversibel gebunden ist.
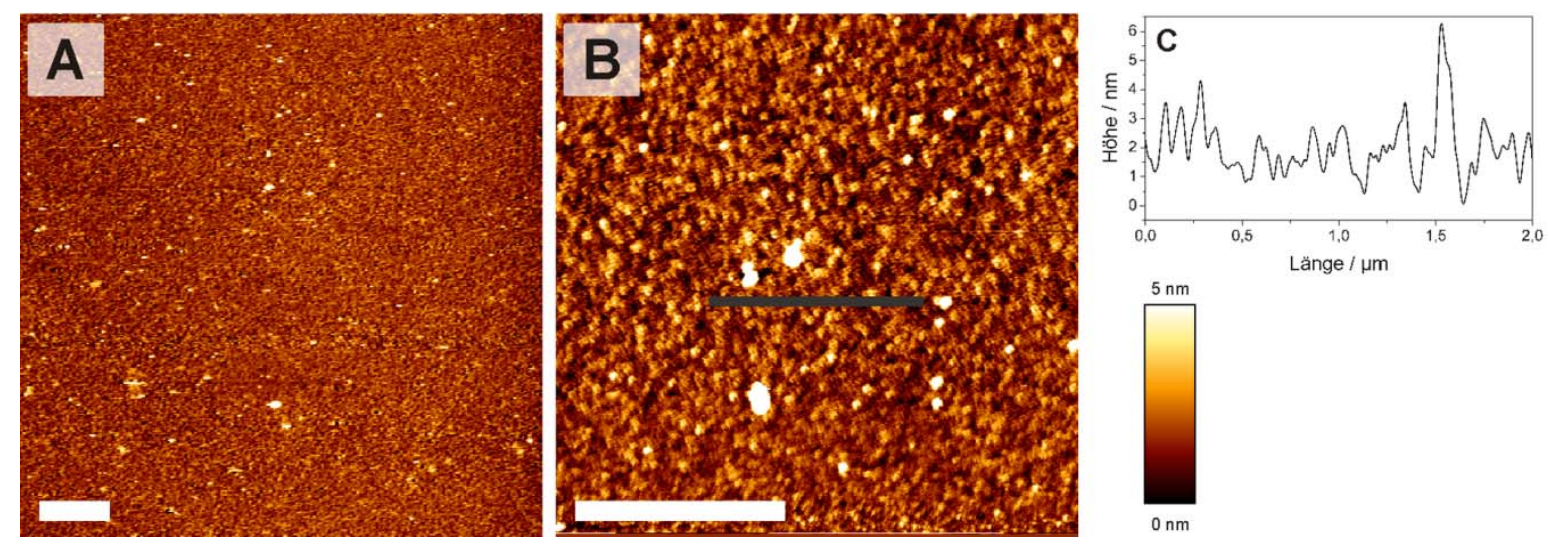

Abbildung 6.9 A/B Topografische AFM-Aufnahmen von Lysinollipidmembranen gespreitet auf Si-Wafern (100 nm SiO 2 -Schicht). Die Proben wurden mit $50 \mathrm{~mm} \mathrm{Si}(\mathrm{OH})_{4}$ für $1 \mathrm{~h}$ in $50 \mathrm{~mm} \mathrm{MES} \mathrm{pH} \mathrm{6,0} \mathrm{inkubiert} \mathrm{und}$ im Anschluss mit Puffer und Reinstwasser gespült. Gemessen wurde in Reinstwasser im tapping-Modus (NWII) mit MSCT-Au cantilevern. Maßstabsbalken: $2 \mu \mathrm{m}$. C Höhenprofil entlang der schwarzen Linie in Aufnahme B.

\section{Silicastrukturen an Lysinollipidschichten (LB-Übertrag)}

Die Fluoreszenzaufnahmen von Monoschichten bestehend aus Lysinollipid, die mittels LB-Technik immobilisiert wurden, verändern sich durch die Zugabe von Monokieselsäure nicht wesentlich. Es ist $\mathrm{zu}$ beachten, dass die in Abbildung $6.10 \mathrm{~A} / \mathrm{B}$ dargestellten Aufnahmen mit Abbildung 6.5 D verglichen werden sollten, da nur bei dieser Aufnahme die DPPC-Monoschicht, die sich unter der Lysinollipidmonoschicht befindet, mit einem Fluoreszenzmarker versetzt war. Weshalb die Fluoreszenz der Lysinollipidschicht dadurch verändert aussieht, erklären Aufnahmen B und C, die beide an einer Stelle aber mit unterschiedlichen Fluoreszenzfiltersätzen (für die jeweilige Wellenlänge der Fluorophore) erstellt wurden: Die Verteilung von $\beta$-BODIPY 500/510 $\mathrm{C}_{12}$-HPC in der DPPC-Schicht ist nicht homogen $(\mathrm{C})$ und durch einen FRET-Effekt wird dadurch auch die Sulforhodamin 101 DHPE-Fluoreszenz (B) beeinflusst. Dort, wo die DPPC-Schicht hell erscheint, ist die Lysinollipid-Fluoreszenz geschwächt. 

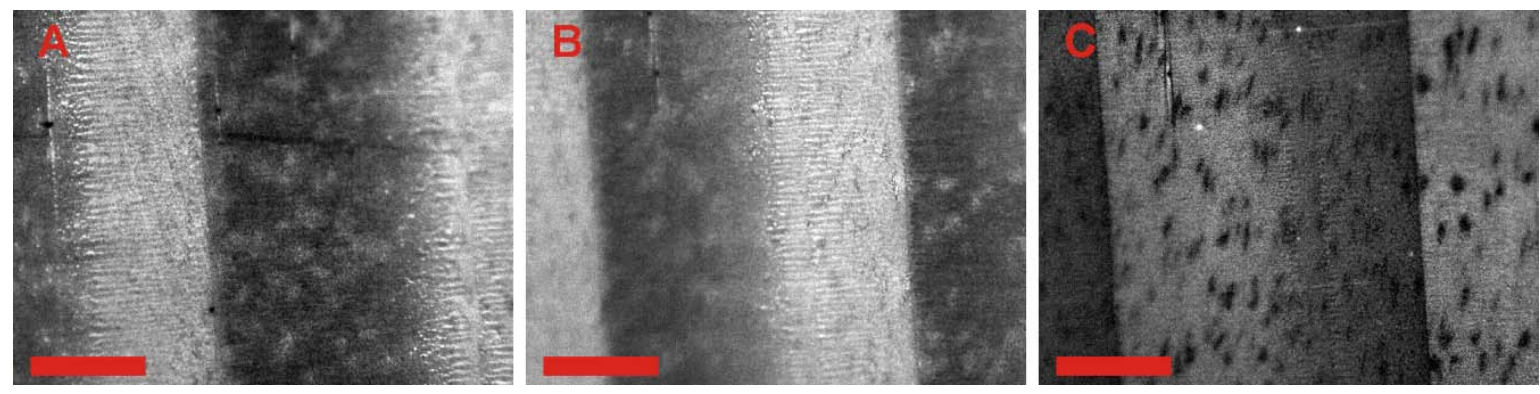

Abbildung 6.10 Fluoreszenzaufnahmen einer bei $\pi=25 \mathrm{mN} / \mathrm{m}$ immobilisierten Lysinollipidmonoschicht (mit $0,2 \mathrm{~mol} \%$ Sulforhodamin 101 DHPE) auf einem Siliciumwafer $\left(100 \mathrm{~nm} \mathrm{SiO}_{2}\right)$ bedeckt mit einer DPPC-Monoschicht $\left(0,5 \mathrm{~mol} \% \quad \beta\right.$-BODIPY 500/510 $\mathrm{C}_{12}$-HPC). Die Probe wurde für eine Stunde mit $\sim 100 \mathrm{mM} \mathrm{Si}(\mathrm{OH})_{4}$ inkubiert und danach mit Puffer und Reinstwasser gespült. A-C Filtersatz 45. D Filtersatz 44. Maßstabsbalken $50 \mu \mathrm{m}$.

Vergleicht man die Aufnahmen der Proben, die mit Kieselsäure inkubiert wurden (Abbildung 6.10 A/B) mit denen vor der Zugabe (Abbildung $6.5 \mathrm{D}$ ) kann man keine Unterschiede feststellen, was darauf schließen lässt, dass die Membranen noch intakt sind. Die Oberfläche wirkt unregelmäßiger, teilweise körnig, es lässt sich jedoch nicht aussagen, wodurch diese Veränderung bedingt wurde.

AFM-Messungen zeigen, dass auch im Fall der durch LB-Übertrag erhaltenen Membranen drastische Veränderungen der Oberflächentextur, ausgelöst durch die Zugabe von Kieselsäure, auftreten. In Abbildung 6.11 sind verschiedene Messergebnisse zusammengestellt, wobei jeweils Aufnahmen A/B/D beziehungsweise $D / E / F$ von einer Probe stammen. Die Rauheit der Oberfläche, ermittelt anhand Aufnahme C, hat sich von $S_{a}=1,6 \mathrm{~nm}$ auf $S_{a}=2,2 \mathrm{~nm}$ erhöht. In Aufnahme A können die vor der Zugabe vorhandenen Oberflächenstrukturen wie Quer- und Längsstreifen sowie runde Defekte (Abbildungen 6.5 und 6.6) wiedergefunden werden, allerdings ist die komplette Oberfläche mit kleinen Partikeln belegt. Vergrößerungen zeigen deutlich runde Objekte, die als Silicakugeln interpretiert werden. Sie weisen einen Durchmesser von ca. 70-80 nm auf(G), jedoch hängt dieser Wert von der jeweiligen Messung ab. Teilweise werden Kugeln mit Durchmessern von bis zu $200 \mathrm{~nm}$ detektiert. Ein Vergleich zwischen Aufnahmen B und C beziehungsweise $\mathrm{D}$ und $\mathrm{E}$, die jeweils gleich große Probenausschnitte wiedergeben, verdeutlicht das. Die Kugeln in B und D, die von einer Probe stammen und mit dem gleichen cantilever gemessen wurden sind deutlich größer, als diejenigen in $\mathrm{C}$ und $\mathrm{E}$, die wiederum von einer Probe stammen mit dem gleichen cantilever vermessen wurden. Ein Grund hierfür kann die Geometrie der cantilever-Spitze sein. Allgemein traten bei den Messungen nach kurzer Zeit Verschmutzungen der Messspitze auf, was die Abbildung erschwerte und eine genaue Größenbestimmung der Silicapartikel unmöglich macht. In Reinstwasser traten diese weniger häufig auf, was der Grund dafür ist, dass nicht in Puffer 
gemessen wurde. In der Regel variierten Partikelgeometrie und -größe leicht von Messung zu Messung. Die Partikel sahen nicht immer rund aus. Es kann nicht unterschieden werden, ob bei jeder Präzipitation leicht unterschiedliche Partikel gebildet wurden, oder ob dieses Phänomen ausschließlich auf die Messspitzen zurückzuführen ist. In Aufnahmen C und E kann unter der körnigen Oberfläche noch die Struktur der LB-Lysinollipidmembran erkannt werden, die durch die Defekte geprägt war (Abbildung 6.6). Eine Histogrammanalyse des blau markierten Ausschnitts in Aufnahme E ergab einen mittleren Höhenunterschied von 4,8 nm und entspricht damit der Membranhöhe vor der $\mathrm{Si}(\mathrm{OH})_{4^{-}}$ Inkubation, die 4,2 $\mathrm{nm}$ betrug.
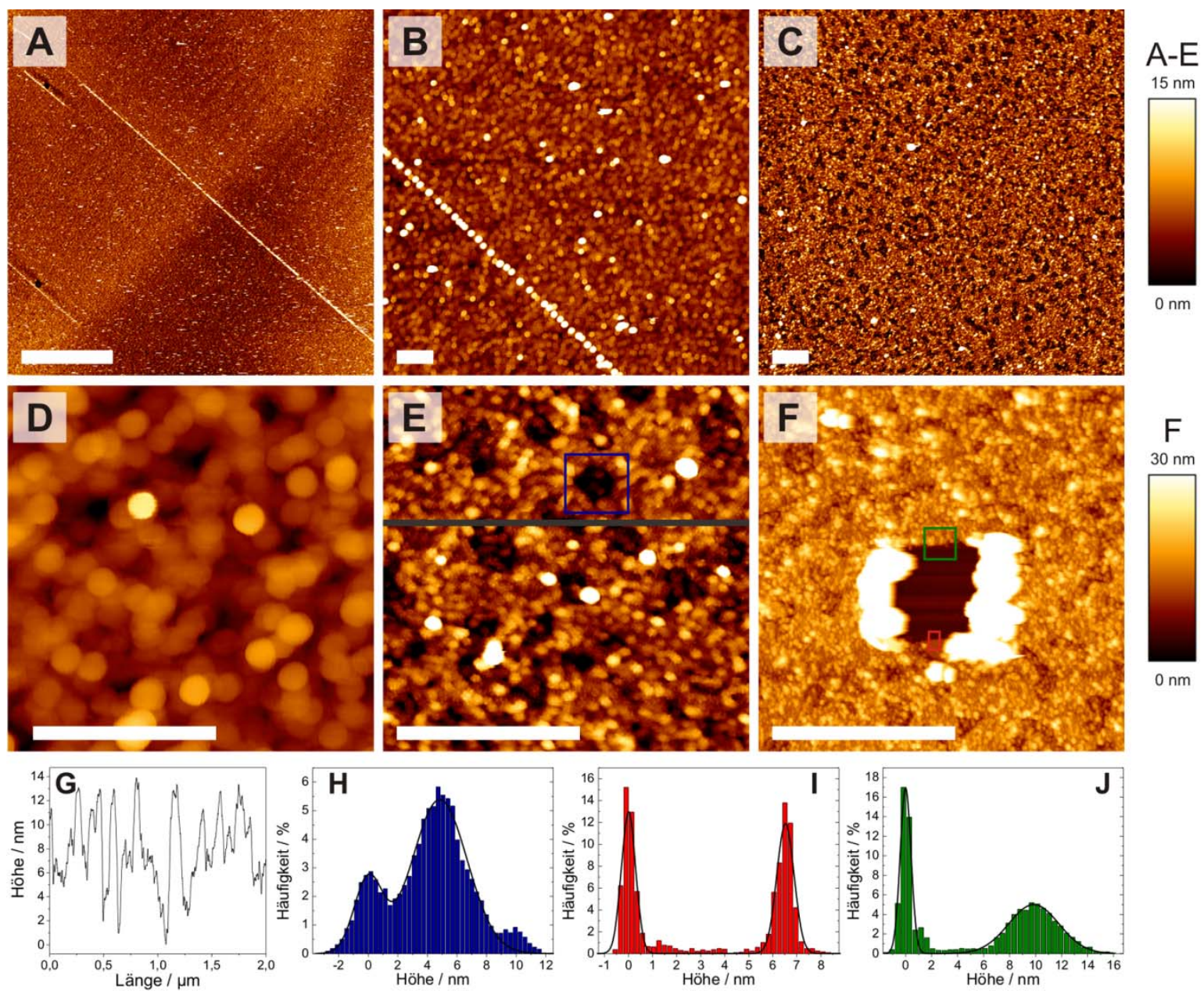

Abbildung 6.11 A-F Rasterkraftmikroskopische Aufnahmen von mittels LB-Übertrag immobilisierten Lysinollipidschichten (Oberflächendruck während des Übertrags: $27 \mathrm{mN} / \mathrm{m}$ ) auf DPPC/Si-Wafern, die für $1 \mathrm{~h}$ mit $\sim 100 \mathrm{mM} \mathrm{Si}(\mathrm{OH})_{4}$ in $50 \mathrm{~mm}$ MES pH 6,0 versetzt wurden. Die Proben wurden mit Puffer und Reinstwasser gespült. Die tapping mode AFM-Messungen (NWI \& NWII) in Reinstwasser wurden mit A/B/D CSC37- beziehungsweise C/D/F MSCT-Au-cantilevern durchgeführt. Maßstabsbalken: A: $20 \mu \mathrm{m}$, B-F: $1 \mu \mathrm{m}$. G Höhenprofil entlang der schwarzen Linie in E. H-J Histogrammanalysen der Höhen in den jeweils farbig markierten Bereichen. 
Somit kann davon ausgegangen werden, dass die Bildung von Silica auf der kompletten Oberfläche, auch in den Defekten, stattgefunden hat und diese mit einer überall ungefähr gleich hohen Schicht überzogen hat. Um die Dicke dieser Silicaschicht zu bestimmen, wurde das Material mit der Messspitze bei hoher applizierter Kraft von der Oberfläche entfernt $(\mathrm{F})$. In Aufnahme $\mathrm{F}$ ist ein Bereich von $(500 \times 500) \mathrm{nm}$ dargestellt, in dem die Oberfläche des Siliciumwafers freigelegt wurde. Der rot markierte Bereich umfasst ein glattes Plateau mit einer Höhe von ca. 6,5 nm (I), das die Oberfläche der Doppelschicht ohne Silica darstellt. Vergleicht man damit den Höhenunterschied im grünen Bereich, also zu einer mit Silica bedeckten Fläche, der ca. 9,9 nm beträgt (J), kann aus der Differenz die Silicaschichtdicke abgeschätzt werden. Sie beträgt demnach ca. (3 - 4) nm, was im gleichen Größenbereich liegt, wie die Höhe der in den Höhenprofilen erkennbaren Objekte (Abbildungen 6.9 und 6.11). Es handelt sich dabei nicht um eine gleichmäßige Höhe, da wie in den AFM-Aufnahmen ersichtlich wird, die Schicht aus einzelnen Partikeln besteht. Bei zusammenfassender Betrachtung der vorgestellten Ergebnisse kann gefolgert werden, dass an der Oberfläche von Lysinollipidschichten die Polymerisation von Kieselsäure ausgelöst wird. Der Prozess liegt in einer spezifischen Wechselwirkung der Aminogruppen mit der Kieselsäure begründet. Die gesamte Oberfläche wird, unabhängig von der Präparationsmethode der Membran, mit einer Schicht bestehend aus Silicapartikeln bedeckt, die weniger als $10 \mathrm{~nm}$ dick ist. Weiterhin zeigten die Fluoreszenzaufnahmen, dass die Lipidschicht unterhalb des Silica weitestgehend nicht verändert wurde.

\section{Stabilität der Präzipitate bei Trocknen}

Eine weitere interessante Fragestellung ist, wie sich die Oberflächen beim Trocknen der Probe verändern. $\mathrm{Zu}$ diesem Zweck wurde das Wasser über Lysinollipidschichten, die mittels LB-Technik präpariert wurden, mit darauf präzipitiertem Silica abpipettiert, die Proben an Luft getrocknet und im Anschluss untersucht. Fluoreszenzmikroskopische Aufnahmen geben dabei keinen Hinweis auf eine eventuelle Veränderung (Abbildung 6.12). Die Bilder ähneln denen, die unter Wasser erstellt wurden, sowohl mit als auch ohne präzipitiertem Silica (Abbildung 6.5, Abbildung 6.10). Die durch die Immobilisierung bedingten Streifen sind auch an Luft teilweise sichtbar (A), in anderen Bereichen ist die Fluoreszenz homogen (B). Stellenweise kann die Bildung von einzelnen (B) beziehungsweise häufig auftretenden (C) dunklen Stellen beobachtet werden. 

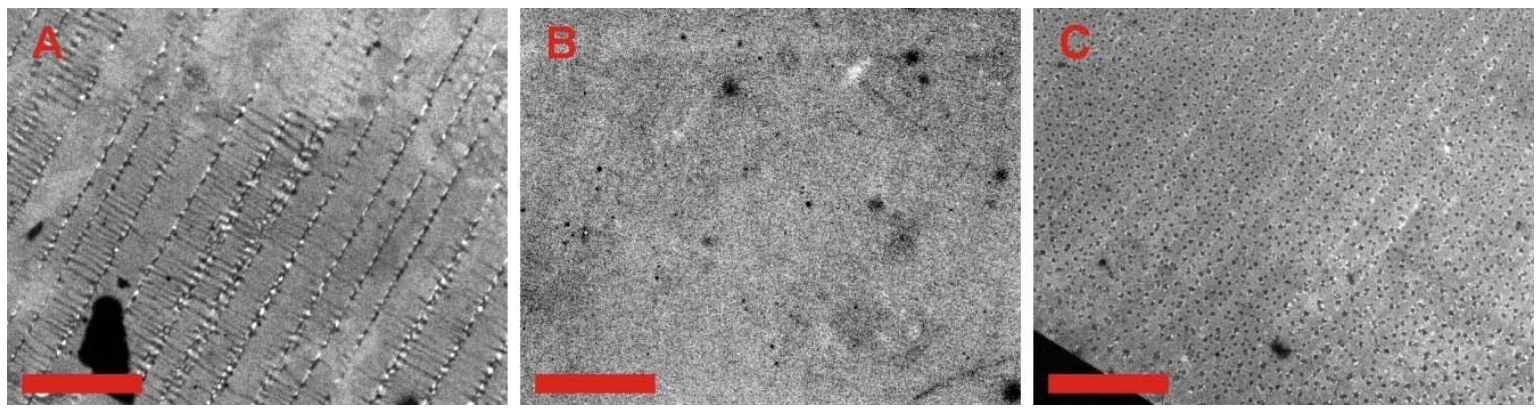

Abbildung 6.12 A-C Fluoreszenzbilder von Lysinollipid (0,5 mol\% Sulforhodamin 101 DHPE) bei $\pi=27 \mathrm{mN} / \mathrm{m}$ mittels LB-Technik immobilisiert auf DPPC/Siliciumwafern (100 nm Oxid) und $60 \mathrm{~min}$ inkubiert mit $\sim 100 \mathrm{mM} \mathrm{Si}(\mathrm{OH})_{4}$ in $50 \mathrm{mM}$ MES pH 6,0. Die Proben wurden mit Reinstwasser gewaschen und anschließend an Luft getrocknet. Die Aufnahmen wurden an Luft erstellt. Maßstabsbalken: $100 \mu \mathrm{m}$.

Die getrockneten Proben wurden zudem rasterkraftmikroskopisch an Luft untersucht. Die dabei abgebildeten Oberflächen sind in Abbildung 6.13 dargestellt. Vergleicht man diese mit den in Abbildung 6.11 aufgeführten Aufnahmen, kann kein Unterschied festgestellt werden, was darauf hinweist, dass sowohl die Lipidschichten als auch das Silica noch auf der Oberfläche vorhanden sind. Die Rauheit der Probe wurde anhand Aufnahme B ermittelt und beträgt $S_{a}=1,8 \mathrm{~nm}$, ist also um 0,4 nm kleiner als vor dem Trocknen. Die Partikel besitzen Durchmesser von (70-200) nm und eine Höhe von ca. $4 \mathrm{~nm}$, was auch aus dem Höhenprofil (C) ersichtlich wird.
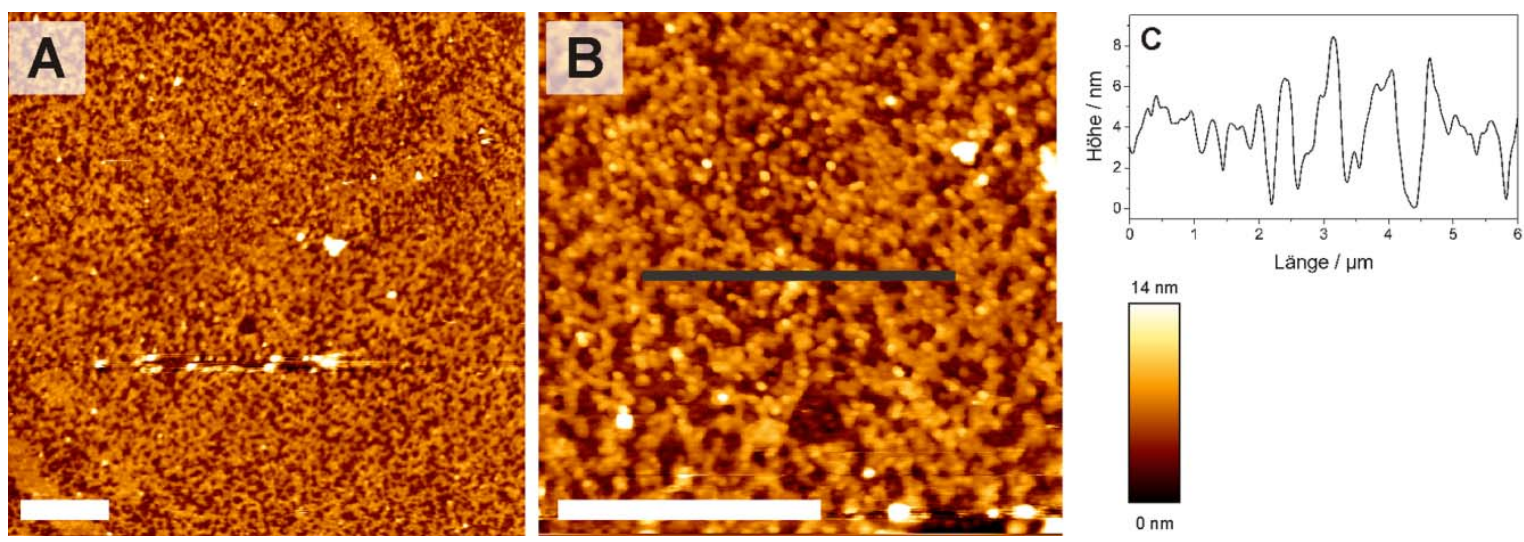

Abbildung 6.13 A/B AFM-Aufnahmen: Lysinollipidmonoschichten, mittels LB-Technik bei $\pi=27 \mathrm{mN} / \mathrm{m}$ übertragen auf DPPC-beschichtete Si-Wafer, wurden in $50 \mathrm{mM}$ MES pH 6,0 für $1 \mathrm{~h}$ mit $\sim 100 \mathrm{mM} \mathrm{Si}(\mathrm{OH})_{4}$ inkubiert, mit Reinstwasser gespült und getrocknet. Die Messungen wurden an Luft im tapping-Modus (NWII) mit Pointprobe NCH-cantilevern durchgeführt. Maßstabsbalken: $5 \mu \mathrm{m}$. C Höhenprofil entlang der schwarzen Linie in Aufnahme B.

Das Trocknen der Proben erlaubt eine elektronenmikroskopische Untersuchung, die weitere Informationen über die Probenoberfläche liefert. Hierzu wurden die Proben ohne weitere Behandlung über leitende Klebeplättchen auf SEM-Probenhaltern fixiert und die Oberfläche leitend über Silberleitlack kontaktiert, bevor sie vermessen wurden. Die dabei 
entstandenen Aufnahmen sind in Abbildung 6.14 aufgeführt. Bei kleinen Vergrößerungen (A/B) kann man, wie schon in den Fluoreszenz- und AFM-Bildern, die streifige Oberflächenstruktur erkennen. Durch Vergleich der SEM-Aufnahmen mit den AFM- und Fluoreszenzbildern können die hellen Bereiche in den SEM-Aufnahmen der Lipidschicht zugeordnet werden, während die Defekte dunkel erscheinen. Erst bei hoher Auflösung (E/F) sind auf der Oberfläche kleine Partikel erkennbar. Diese bedecken sowohl die hellen als auch die dunklen Regionen, sind jedoch nicht völlig gleichmäßig verteilt, vielmehr gibt es Anhäufungen (F). Die Durchmesser können zwar nicht genau bestimmt werden, Aufnahmen $\mathrm{E}$ und $\mathrm{F}$ deutet jedoch darauf hin, dass sie unter $10 \mathrm{~nm}$ liegen und somit kleiner sind, als in den AFM-Messungen ermittelt wurde. Geht man davon aus, dass die Silicapartikel rund sind und eine Silicamonoschicht auf der Oberfläche gebildet wurde, sollte der Durchmesser der Kugeln in etwas der Höhe der Schicht entsprechen. Da die Schichtdicke im Bereich von (1-10) nm bestimmt wurde, sind dementsprechend die mittels SEM bestimmten Durchmesser durchaus sinnvoll.
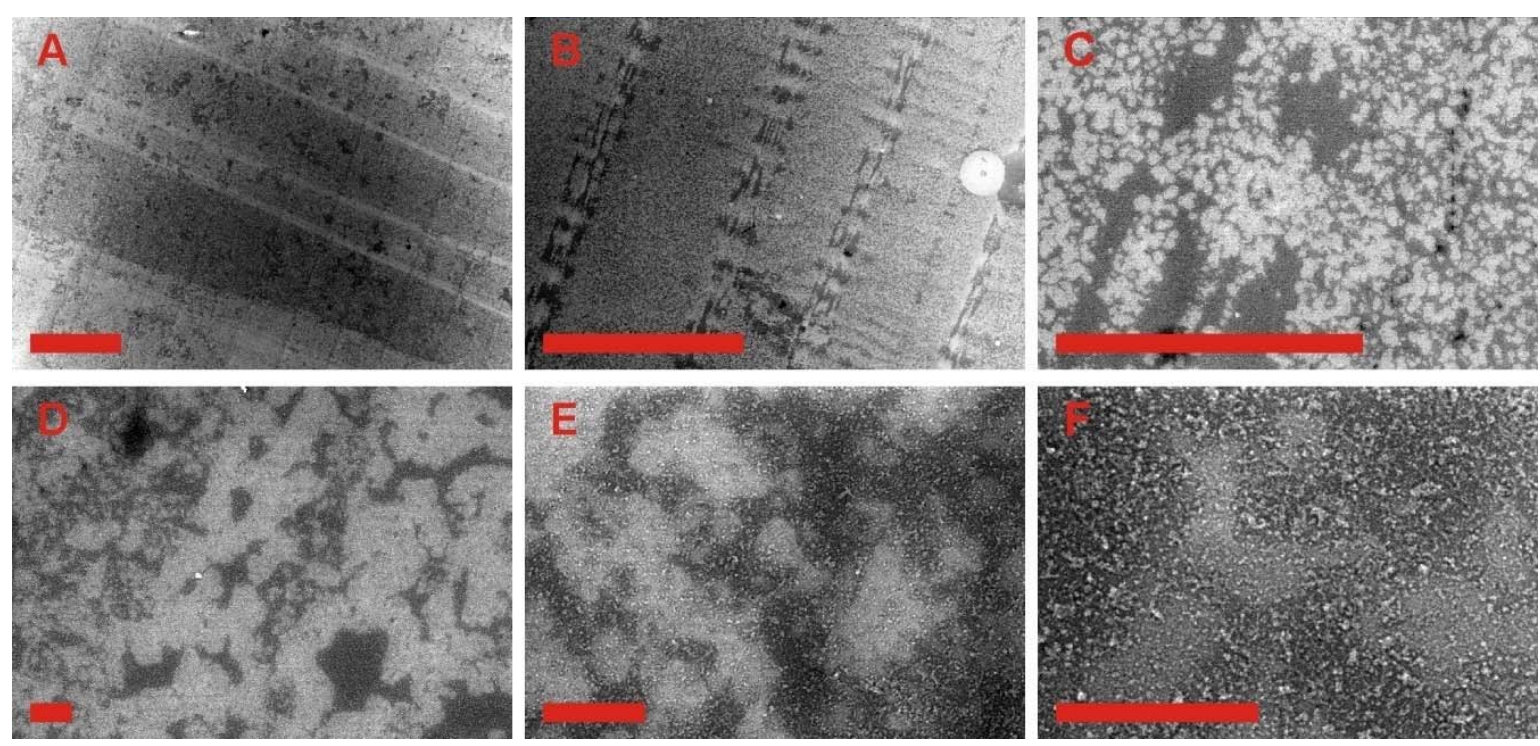

Abbildung 6.14 A-F Elektronenmikroskopische Untersuchung von Lysinollipidschichten, mittels LBTechnik übertragen auf DPPC/Si-Wafer $(\pi=27 \mathrm{mN} / \mathrm{m})$, die für eine Stunde mit $\sim 100 \mathrm{mM} \mathrm{Si}(\mathrm{OH})_{4}$ in $50 \mathrm{~mm}$ MES pH 6,0 versetzt wurden. Die Proben wurden mit Reinstwasser gespült, getrocknet und auf SEMProbenhaltern fixiert. Maßstabsbalken: A-C: $50 \mu \mathrm{m}, \mathrm{D}-\mathrm{F}: 500 \mathrm{~nm}$. 


\subsection{Das System Lysinollipid/C16-Glycerol- $\beta$-Lactosid}

Die Tatsache, dass an planaren Doppelschichten prinzipiell Präzipitationsreaktionen stattfinden und dabei mit der Oberfläche verankerte Silicaschichten entstehen (Kapitel 6.1), war Voraussetzung für das weitere Vorgehen. Im nächsten Schritt sollten binäre Lipidschichten, bestehend aus dem Lysinollipid und einem geeigneten zweiten Lipid, verwendet werden. Ziel war es, eine definierte Struktur zu erzeugen, indem die zweite Komponente in Membrandomänen angereichert wird. Diese Verbindung sollte, im Gegensatz zum Lysinollipid, keine Präzipitationsreaktionen auslösen, wodurch die Silicabildung stellenweise ausbleiben und daraus resultierend eine poröse Silicaschicht gebildet werden sollte. Die schrittweise Umsetzung und Untersuchung des erläuterten Konzepts wird nachfolgend vorgestellt.

\subsubsection{C16-Glycerol- $\beta$-Lactosid}

Das besondere Lipid, welches in Mischung mit dem Lysinollipid verwendet werden kann, muss einige Eigenschaften mitbringen. Zunächst darf es keine silicapräzipitierenden Eigenschaften aufweisen. Weiterhin muss es eine Molekülstruktur aufweisen, die eine dichte Packung in lamellaren Schichten begünstigt, damit das Lipid in Membrandomänen angereichert wird, während das Lysinollipid in der die Domänen umgebenden Matrix verteilt ist. Dabei kristallisierte sich eine Struktur mit einem Disaccharid in der Kopfgruppe und zwei langen gesättigten Alkylresten als hydrophoben Teil als vielversprechend heraus. Eine solche Verbindung besitzt keine Aminogruppen und weist weder positive noch negative Ladungen auf, sollte demnach keine Silicabildung induzieren. Durch die Molekülgeometrie, die durch die zwei Zuckergruppen und die zwei Alkylketten definiert ist, müsste die Bildung lamellar Strukturen gegenüber der von mizellaren Aggregaten bevorzugt sein. ${ }^{172-174}$ Verhältnismäßig lange Ketten mit jeweils 16 Kohlenstoffeinheiten sollten eine dichte Packung und damit die Domänenbildung begünstigen. ${ }^{175,176}$ Die Struktur der eigens für diesen Zweck synthetisierten Verbindung C16-Glycerol- $\beta$-Lactosid (C16-GL) ist in Abbildung 6.15 dargestellt. Die hydrophoben Ketten des Amphiphils sind mit der hydrophilen Kopfgruppe, wie auch bei den Aminolipiden (Tabelle 5.1), mittels Etherbindungen über ein Glycerinmolekül verknüpft. Die molare Masse des Moleküls $\left(\mathrm{C}_{47} \mathrm{H}_{92} \mathrm{O}_{13}\right)$ beträgt $865 \mathrm{~g} / \mathrm{mol}$. 


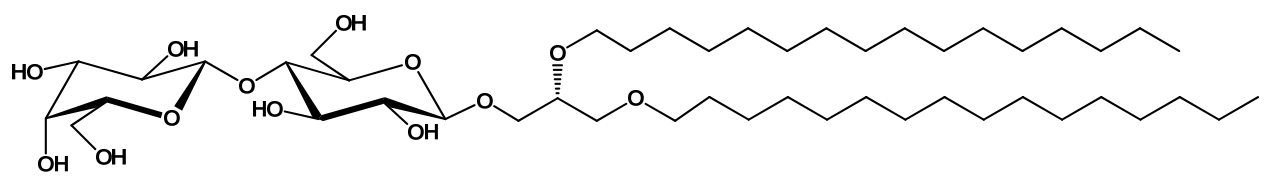

Abbildung 6.15 Strukturformel des Glykolipids C16-Glycerol- $\beta$-Lactosid.

Um auszuschließen, dass C16-GL Silicabildung auslöst, wurde wie mit den Aminolipiden (Kapitel 5.3) ein Präzipitationstest durchgeführt. Zu diesem Zweck wurden zu C16-GLVesikeln in $50 \mathrm{~mm}$ MES pH 6,0 $100 \mathrm{mM} \mathrm{Si}(\mathrm{OH})_{4}$ zugegeben, die Proben für zehn Minuten inkubiert, zentrifugiert und anschließend mit Natronlauge versetzt. Dieser Test erlaubt, mittels $\beta$-Molybdatmethode eventuell präzipitiertes Silica nachzuweisen. Im Fall von C16-GL konnte keine Silicabildung beobachtet werden.

\subsubsection{Lysinollipid/C16-Glycerol- $\beta$-Lactosid-Monoschichten an der Grenzfläche Wasser/Luft}

Um mit der binären Lipidmischung eine wohldefinierte Domänenstruktur produzieren zu können, wurden zunächst Informationen zum Phasenverhalten gesammelt. Zu diesem Zweck wurden Monoschichten bestehend aus Lysinollipid und C16-GL mittels Filmwaagemessungen und damit kombinierter Fluoreszenzmikroskopie untersucht. Zunächst wurden $\pi / A$-Isothermen der beiden reinen Substanzen und ausgewählter Mischungen aufgenommen (Abbildung 6.16 A). Dabei zeigte sich, dass Lysinollipidmonoschichten weniger dicht gepackt sind, als aus C16-GL bestehende, Lysinollipid weist bei gleichem Oberflächendruck eine größere Molekülfläche auf. Betrachtet man beispielsweise die Isothermen der beiden Verbindungen bei einem Oberflächendruck von $\pi \approx 30 \mathrm{mN} / \mathrm{m}$, ergibt sich im Fall von Lysinollipid eine Molekülfläche von $53 \AA^{2}$, die von C16-GL beträgt dagegen nur $31 \AA^{2}$. Der jeweilige Verlauf der $\pi / A$-Isothermen deutet auf unterschiedliches Phasenverhalten der beiden Komponenten hin. Die Lysinollipidisotherme weist bei einem Oberflächendruck von $\pi \approx 39 \mathrm{mN} / \mathrm{m}$ einen Knick auf, der den Übergang von flüssigexpandierter $\left(l_{\mathrm{e}}\right) \mathrm{zu}$ flüssig-kondensierter $\left(l_{\mathrm{c}}\right)$ Phase markiert. Dahingegen deutet die Isotherme von C16-GL darauf hin, dass der Film über den gesamten Bereich in der $l_{\mathrm{c}^{-}}$ beziehungsweise festanalogen $(s)$ Phase vorliegt. Die Isothermen der Mischungen liegen zwischen denen der reinen Verbindungen und weisen bei jeweils unterschiedlichen Oberflächendrücken ebenfalls Knicke auf, auch hier treten dementsprechend Phasenübergänge von $l_{\mathrm{e}} \mathrm{zu} l_{\mathrm{c}}$ auf. Mit steigendem Anteil an Lysinollipid werden diese zu höheren Oberflächendrücken hin verschoben. Diese Abhängigkeit des Phasenübergangs 
von der Zusammensetzung spricht für eine zumindest partielle Mischbarkeit der Komponenten bis zu $\pi_{\mathrm{c}}$, also dem Oberflächendruck, bei dem der $l_{\mathrm{e}}-\mathrm{zu} l_{\mathrm{c}}$-Phasenübergang auftritt. $^{177}$
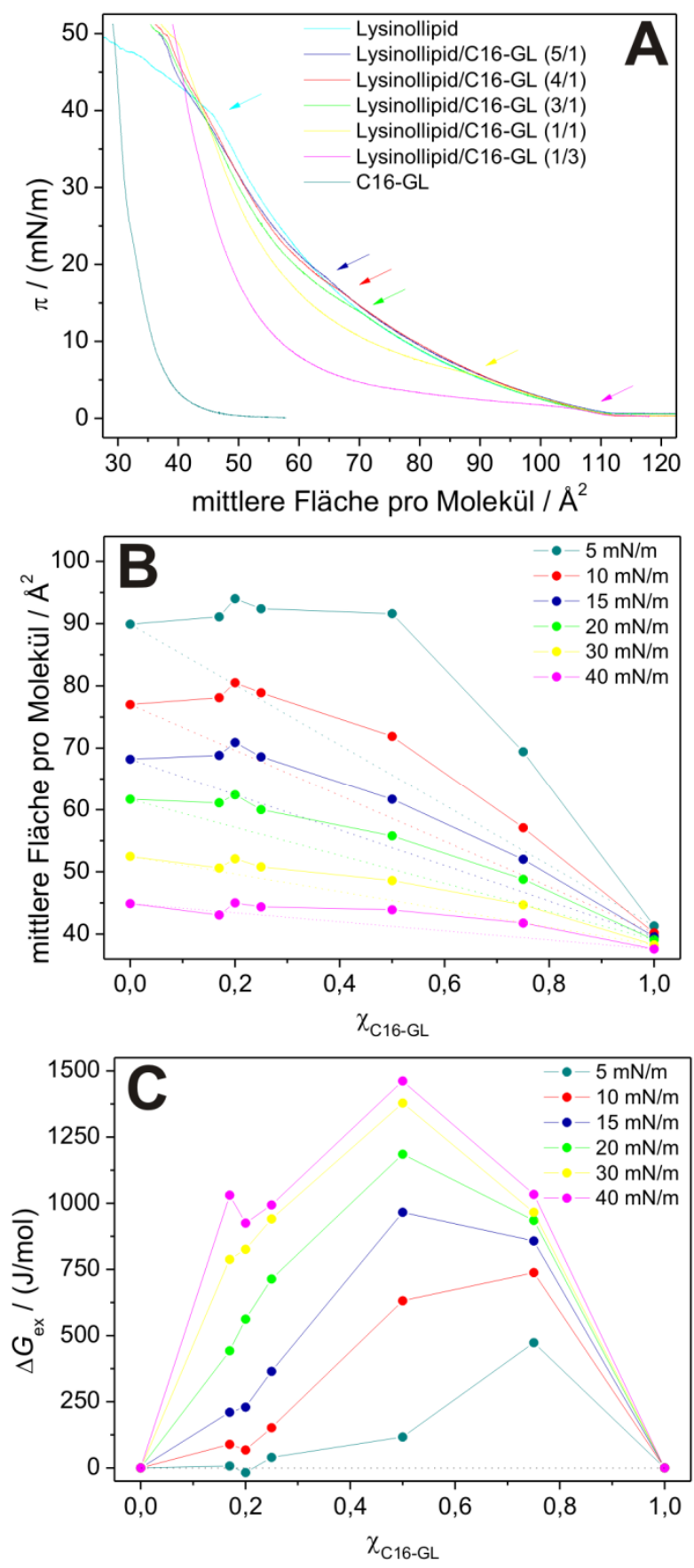

Abbildung 6.16 A $\pi / A$-Isothermen von Mischungen aus Lysinollipid und C16-GL $(n / n)$ an der Grenzfläche Wasser/Luft gemessen bei $21^{\circ} \mathrm{C}$. Die Pfeile markieren den jeweiligen Übergang von $l_{\mathrm{e}}-\mathrm{zu} l_{\mathrm{c}}$-Phase. B Mittlere molekulare Fläche von Lysinollipid/C16-GL-Monoschichten als Funktion des Stoffmengenanteils $\chi_{\text {C16-GL }}$ bei verschiedenen Oberflächendrücken. Die gestrichelten Linien geben jeweils die errechnete mittlere Molekülfläche im Falle unmischbarer beziehungsweise ideal mischbarer Komponenten wieder. C Molare freie Exzess-Oberflächenenergie $\left(\Delta G_{\mathrm{ex}}\right)$ in Abhängigkeit des Molenbruchs $\chi_{\mathrm{C} 16-\mathrm{GL}}$ für das System Lysinollipid/C16-GL. 
Zur näheren Untersuchung der Mischbarkeit der beiden Komponenten in Monoschichten wurde für verschiedene Oberflächendrücke die gemessene mittlere Fläche pro Molekül gegen den Stoffmengenanteil an C16-GL ( $\left.\chi_{\mathrm{C} 16-\mathrm{GL}}\right)$ aufgetragen (Abbildung 6.16 B). Sind die zwei Komponenten vollständig entmischt oder ideal gemischt, so nimmt ein gemischter Film eine Fläche ein, die der Summe aus den Flächenanteilen der einzelnen Komponenten entspricht. Umgekehrt sprechen Abweichungen von diesem Verhalten für zumindest teilweise Mischbarkeit in einem Film. ${ }^{178,179}$

Für das untersuchte System treten bei Oberflächendrücken von $5 \mathrm{mN} / \mathrm{m}$ bis $20 \mathrm{mN} / \mathrm{m}$ positive Abweichungen von den errechneten Flächen auf, während ab $30 \mathrm{mN} / \mathrm{m}$ die errechneten und beobachteten Flächen nahezu übereinstimmen. Dementsprechend sind die Komponenten bei niedrigen Oberflächendrücken zumindest partiell mischbar, bei höheren Drücken hingegen liegt entweder ideale Mischbarkeit beziehungsweise völlige Ummischbarkeit vor, was mit den anhand der Isothermen aufgestellten Schlussfolgerungen übereinstimmt.

Um zusätzliche Informationen zum Prozess der Mischung und zu Wechselwirkungen zwischen den Komponenten zu erhalten, ${ }^{180}$ wurde die molare freie Exzess-Oberflächenenergie $\Delta G_{\mathrm{ex}}$ durch Integration der $\pi / A$-Isothermen bis zu definierten Oberflächendrücken ermittelt und gegen den Stoffmengenanteil an C16-GL $\left(\chi_{\mathrm{C} 16-\mathrm{GL}}\right)$ aufgetragen (Abbildung 6.16 C). Bei fast allen Oberflächendrücken und Mischungsverhältnissen liegen positive Werte von $\Delta G_{\text {ex }}$ vor. Dies bedeutet, dass die Wechselwirkungen zwischen Molekülen der beiden Komponenten geringer sind als zwischen Molekülen einer Komponente, was die Ausbildung zweidimensionaler Aggregate der einzelnen Komponenten fördert. ${ }^{177,178,181}$ Die Zunahme der Werte von $\Delta G_{\text {ex }}$ mit steigendem Oberflächendruck weist auf eine verstärkte Tendenz zur Entmischung hin.

Das Mischungsverhalten der beiden Substanzen kann durch fluoreszenzmikroskopische Messungen weitergehend untersucht werden. Weist das System Lysinollipid/C16-GL Tendenzen zur Entmischung und Bildung zweidimensionaler Aggregate auf, kann dies durch Fluoreszenzfarbstoffe visualisiert werden, die sich in verschiedenen Phasen unterschiedlich verteilen. Ein solcher Farbstoff ist Sulforhodamin 101 DHPE. Es ist bekannt, dass er bei Koexistenz von beispielsweise $l_{\mathrm{e}}$ - und $l_{\mathrm{c}}$-Phase aus der dichter gepackten, also der $l_{\mathrm{c}}$-Phase verdrängt wird und sich überwiegend in der $l_{\mathrm{e}}$-Phase wiederfindet, da seine Löslichkeit hier größer ist als in der $l_{\mathrm{c}}$-Phase. ${ }^{182}$ Für das System Lysinollipid/C16-GL wurden neben reinen Filmen der beiden Substanzen mehrere binäre Mischungen untersucht. Im Fall des Lysinollipids zeigt der Film bei niedrigen Oberflächendrücken eine 
homogene Fluoreszenz (Abbildung $6.17 \mathrm{~A}$ ), bevor ab $\pi \approx 37 \mathrm{mN} / \mathrm{m}$ dunkle Domänen auftreten (Abbildung $6.17 \mathrm{~B})$. Im gleichen Bereich $(\pi \approx 39 \mathrm{mN} / \mathrm{m})$ weist die Lysinollipidisotherme einen Knick auf (Abbildung 6.16 A). Diese Ergebnisse festigen die Hypothese eines Phasenübergangs, ab dem flüssig-kondensierte Domänen und eine flüssigexpandierte Matrix koexistieren. Bei weiterer Erhöhung des Oberflächendrucks nehmen die Domänen größere Anteile der Gesamtfläche in Anspruch (Abbildung 6.17 C), genauer $62 \%$ bei $42 \mathrm{mN} / \mathrm{m}$ im Gegensatz zu $11 \%$ bei $37 \mathrm{mN} / \mathrm{m}$ (Ermittlung der Belegung durch Pixelanalyse mit der Software Scanning Probe Image Processor). Die Isotherme von C16-GL (Abbildung 6.16 A) deutet darauf hin, dass sich die Filme bei allen Oberflächendrücken in der $l_{\mathrm{c}^{-}}$oder festanalogen $(s)$-Phase befinden. Die Fluoreszenzaufnahmen liefern ähnliche Erkenntnisse. Bei allen Oberflächendrücken wurden stark inhomogene Oberflächen beobachtet, die eine geringe Beweglichkeit auf der Subphase aufwiesen (Abbildung 6.17 D).
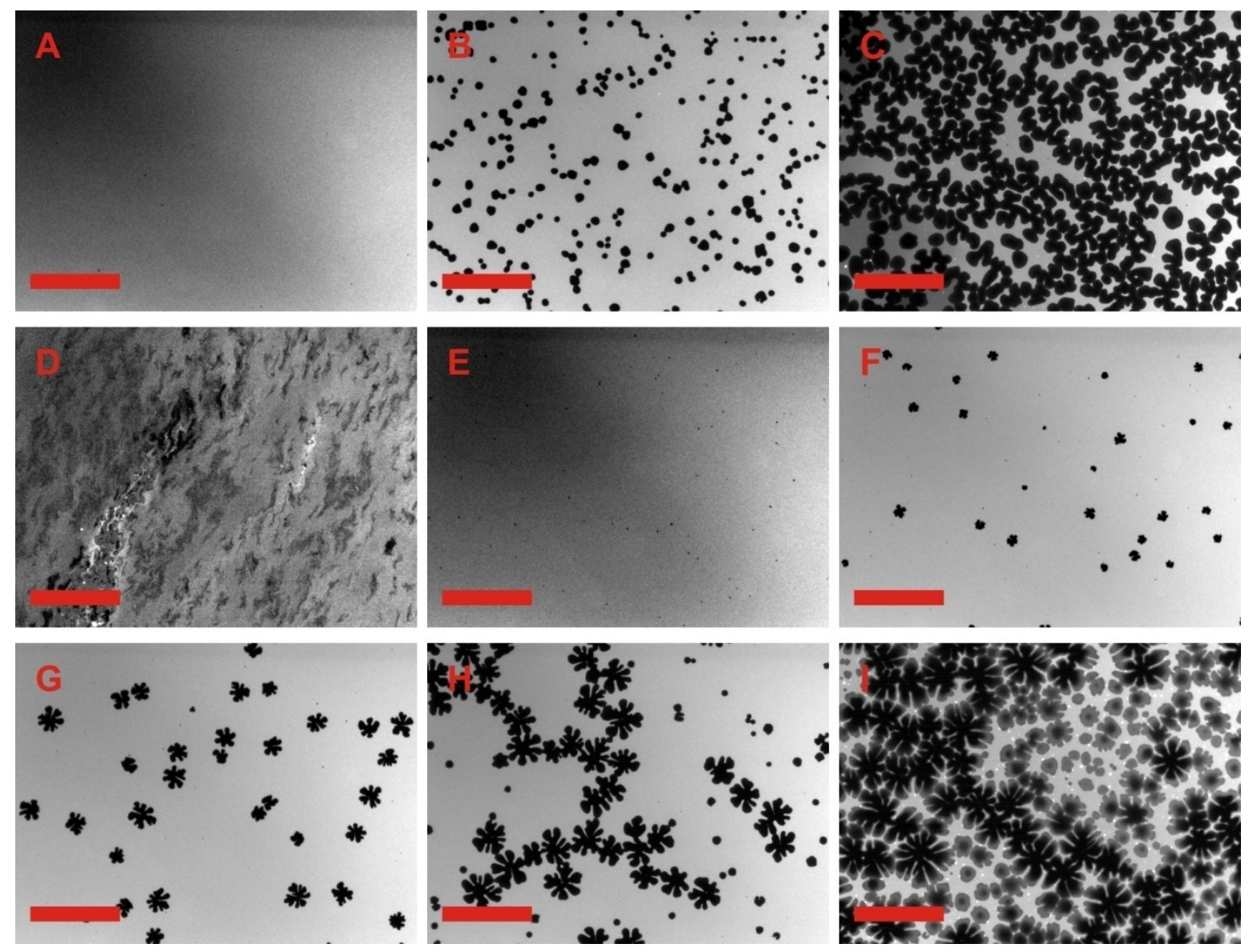

Abbildung 6.17 Fluoreszenzaufnahmen von Monoschichten an der Wasser/Luft-Grenzfläche bestehend aus A-C Lysinollipid ( $\pi$ : A $32 \mathrm{mN} / \mathrm{m}$, B $37 \mathrm{mN} / \mathrm{m}$, C $42 \mathrm{mN} / \mathrm{m}$ ), D C16-GL bei $\pi=20 \mathrm{mN} / \mathrm{m}$ beziehungsweise E-I Lysinollipid/C16-GL 4/1 (n/n) ( $\pi$ : E $11 \mathrm{mN} / \mathrm{m}$, F 15 mN/m, G 20 mN/m, H 30 mN/m und I 44 mN/m) und jeweils $1 \mathrm{~mol} \%$ Sulforhodamin 101 DHPE. Temperatur: $21^{\circ} \mathrm{C}$. Maßstabsbalken: $100 \mu \mathrm{m}$. 
In binären Filmen der Mischungsverhältnisse Lysinollipid/C16-GL = (5/1), (4/1), (3/1) und $(1 / 1)(n / n)$ setzte jeweils ungefähr bei dem Oberflächendruck, bei dem die entsprechende Isotherme einen Knick zeigt (Abbildung 6.16 A), Domänenbildung ein. Exemplarisch sind Fluoreszenzaufnahmen eines Films der Mischung (4/1) dargestellt (Abbildung 6.17 E-I). In diesem Fall wurden Domänen $\mathrm{ab} \pi \approx 15 \mathrm{mN} / \mathrm{m}$ sichtbar, also ab dem Oberflächendruck, bei dem auch die Isotherme auf einen $l_{\mathrm{c}^{-}} \mathrm{zu} l_{\mathrm{e}}$-Übergang hindeutet. Eine Erhöhung des Oberflächendrucks bewirkt eine Vergrößerung der dunklen Domänen (F-H), die mehr und mehr aggregieren $(\mathrm{H})$, bis sich ein Netzwerk ausbildet und die fluoreszierenden Bereiche stark reduziert sind (I). Die Durchmesser der Domänen lagen für diese Mischung im Bereich von 15-27 $\mu \mathrm{m}$. Diese Aufnahmen sprechen zusammen mit den oben diskutierten Aspekten darauf hin, dass mit zunehmendem Oberflächendruck im System ein Entmischungsprozess stattfindet. Das bedeutet, dass in den Domänen eine der beiden Komponenten angereichert wird, die zweite überwiegend in den umgebenden Bereichen zu finden ist. Die Analysen mehrerer Mischungen ergaben, dass sich der Flächenanteil an dunklen Domänen mit steigendem Stoffmengenanteil an C16-GL bis zu einem Oberflächendruck von $\pi=32,5 \mathrm{mN} / \mathrm{m}$ erhöht (Abbildung 6.18).

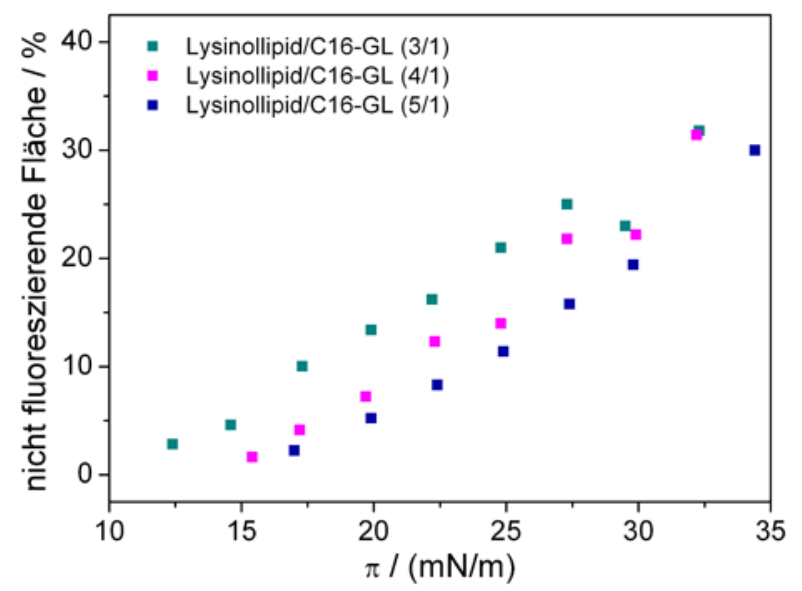

Abbildung 6.18 Variation der nichtfluoreszierenden Fläche mit dem Oberflächendruck in Monoschichten von drei Lysinollipid/C13-GL-Mischungen an der Wasser/Luft-Grenzfläche. Angezeigt wird die beobachtete Flächenbelegung durch Domänen ermittelt über Pixelanalysen entsprechender Fluoreszenzaufnahmen.

Dies spricht für eine Anreicherung des Glykolipids in den Domänen. ${ }^{182}$ Auch in anderen Arbeiten wurde gezeigt, dass sich Lipide, die im reinen Zustand stärker kondensiert sind als der Mischungspartner, in Domänen anreichern. ${ }^{177,183}$ Dieses Ergebnis entspricht der vorweg aufgestellten Hypothese, nach welcher die langen Alkylketten von C16-GL eine dichte Packung und dadurch Domänenbildung bewirken sollten. Weiterhin von Interesse ist, dass die Ergebnisse darauf hindeuten, dass ab Oberflächendrücken von 
$\pi \approx 35-40 \mathrm{mN} / \mathrm{m}$ auch das Lysinollipid einen Phasenübergang durchläuft und Domänen mit Lysinollipid in der $l_{\mathrm{c}}$-Phase auftreten, die neben den mit C16-GL angereicherten vorliegen. Wenn Lysinollipid/C16-GL-Monoschichten für spätere Präzipitationsreaktionen immobilisiert werden, sollte die Entmischung der beiden Komponenten möglichst stark sein. Darüber hinaus sollte ein Langmuir-Blodgett-Übertrag für eine erfolgreiche Durchführung bei möglichst hohem Oberflächendruck durchgeführt werden. Die Untersuchung der unterschiedlich zusammengesetzten binären Filme ergab, dass das Stoffmengenverhältnis Lysinollipid/C16-GL = 4/1 gute Voraussetzungen bietet, sofern der Übertrag im Bereich von $\pi=(25-30) \mathrm{mN} / \mathrm{m}$ durchgeführt wird. Die Entmischung ist laut der Analysen gegeben und der Oberflächendruck ist nicht so hoch, dass auch Lysinol in der $l_{\mathrm{c}}$-Phase vorliegt. Aus diesen Gründen wurde in der Folge mit dieser Mischung gearbeitet.

\subsubsection{Charakterisierung von festkörperunterstützten Lysinollipid/C16-GL-Membranen}

Die fluoreszenzmikroskopische Untersuchung der Lysinollipid/C16-GL-Monoschichten zeigten, dass in den Filmen mit C16-GL angereicherte Domänen vorliegen. Eine Methode solche Monoschichten $\mathrm{zu}$ immobilisieren, stellt die bereits früher erwähnte LangmuirBlodgett (LB)-Technik dar. Dabei müssen Übertragsbedingungen eingehalten werden, die den Erhalt der Morphologie der Monoschichten optimal unterstützen. Wie bereits in Kapitel 6.1 erläutert, eignen sich als Substrate Siliciumwafer oder Glimmer, auf deren hydrophilen Oberflächen ebenfalls durch LB-Übertrag eine 1,2-Dipalmitoyl-sn-glycero-3phosphatidylcholin (DPPC)-Monoschicht abgeschieden wird, wodurch die Oberfläche in der Folge hydrophob ist. In einem zweiten Schritt können dann von der Wasser/LuftGrenzfläche Monoschichten darauf immobilisiert werden. Glimmer bietet im Gegensatz zu Si-Wafern aufgrund seiner teilweisen Lichtdurchlässigkeit, die Möglichkeit kombinierter AFM- und Fluoreszenzuntersuchungen, indem ein inverses Fluoreszenzmikroskop verwendet wird. Wird ein Lysinollipid/C16-GL (4/1)-Film, der einen Fluoreszenzfarbstoff enthält, bei $25 \mathrm{mN} / \mathrm{m}$ auf DPPC/ Glimmer immobilisiert, sind ähnliche Domänenstrukturen erkennbar (Abbildung 6.19 A/B/C), wie in den Monoschichten an der Wasser/LuftGrenzfläche (Abbildung 6.17 G/H). In allen Aufnahmen sind streifenförmige Bereiche unterschiedlicher Morphologie erkennbar, die quer zur Aufziehrichtung verlaufen (z.B. Abbildung 6.19 D). Die fluoreszierenden Flächen sind nicht homogen hell, sondern durchsetzt mit kleinen dunklen Punkten. Die Präparation dieser Membranen funktioniert 
prinzipiell, stellte sich jedoch als nicht immer reproduzierbar heraus. Oftmals wird die Phasenseparation nicht gut erhalten. Zum Teil sind die Umrisse der runden oder blumenförmigen dunklen Domänen nicht mehr gut definiert (C) oder ihre Struktur ist verzerrt (D).
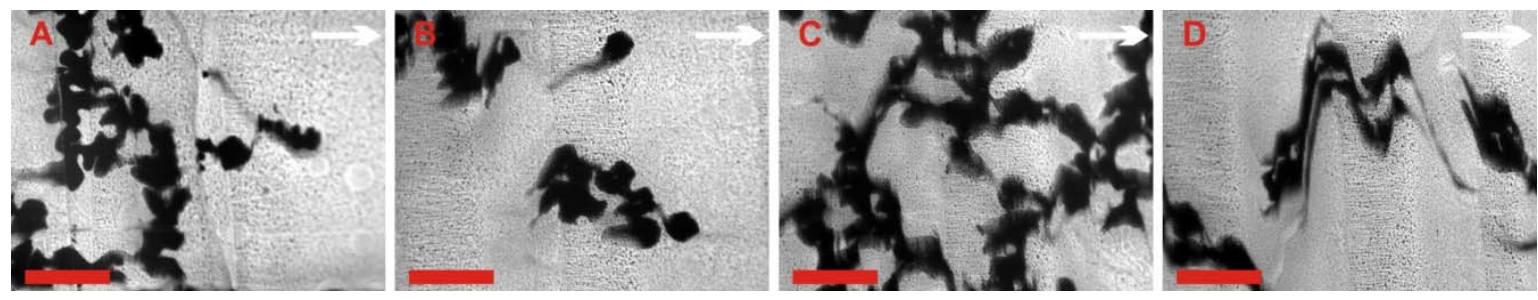

Abbildung 6.19 Fluoreszenzaufnahmen von mittels LB-Technik bei $\pi=25 \mathrm{mN} / \mathrm{m}$ übertragenen Lysinollipid/C16-GL (4/1)-Monoschichten ( $0,5 \mathrm{~mol} \%$ Sulforhodamin 101 DHPE). Als Substrat diente Glimmer beschichtet mit einer DPPC-Monoschicht, die bei $\pi=45 \mathrm{mN} / \mathrm{m}$ immobilisiert wurde. Subphase: Reinstwasser. Maßstabsbalken: $50 \mu \mathrm{m}$. Die Aufziehrichtung ist durch die weißen Pfeile markiert.

Um weitere Informationen über diese Doppelschichten $\mathrm{zu}$ erhalten, wurden sie rasterkraftmikroskopisch untersucht. Abbildung 6.20 gibt eine Übersicht über die auf diese Weise erhaltenen Ergebnisse.
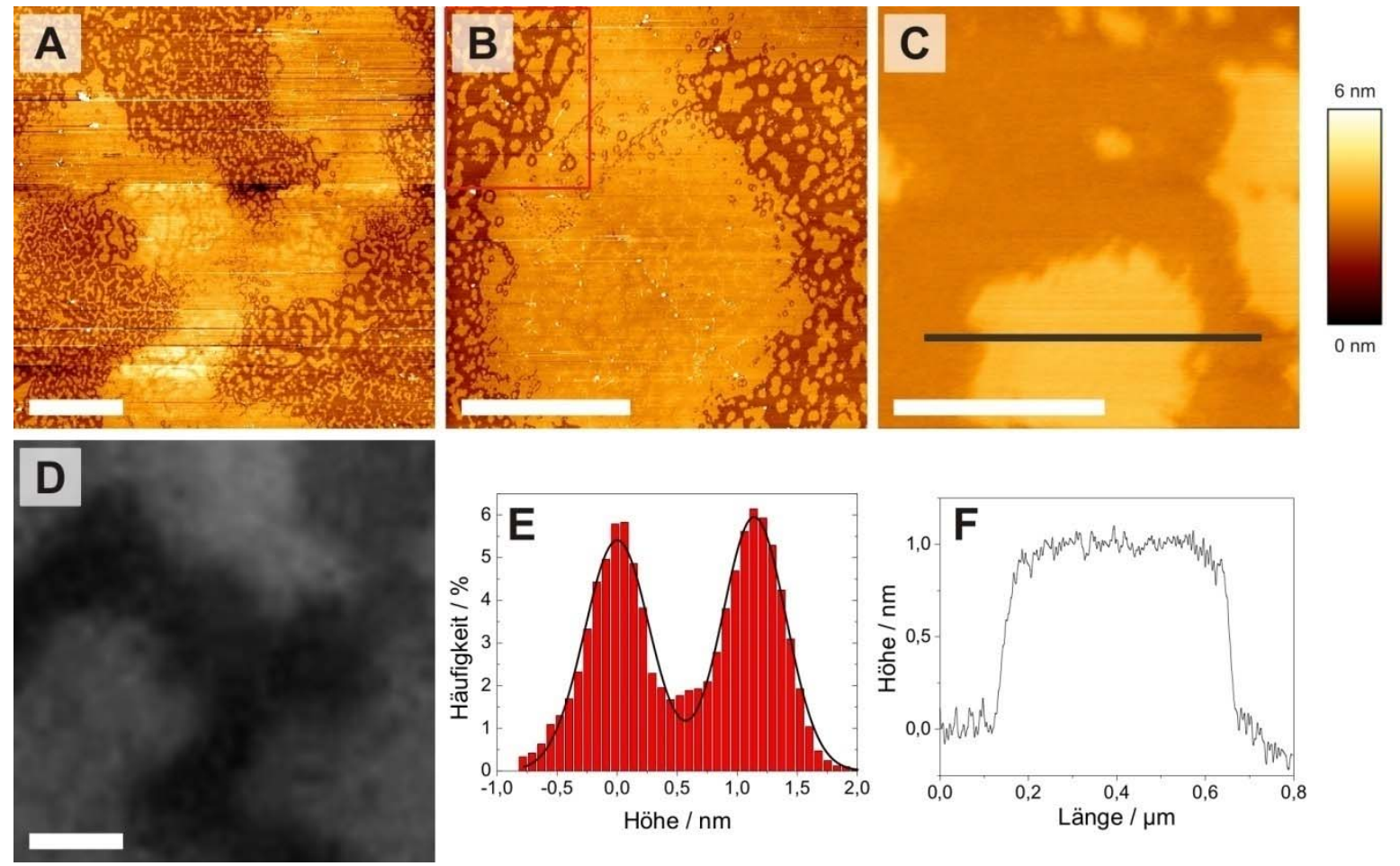

Abbildung 6.20 A-C Topografische AFM-Aufnahmen von Lysinollipid/C16-GL (4/1)-Filmen, die bei $27 \mathrm{mN} / \mathrm{m}$ auf DPPC/Glimmer mittels LB-Technik immobilisiert wurden. AFM-Messungen: tapping-Modus, CSC37-cantilever, NWII, Reinstwasser. D Fluoreszenzmikroskopische Aufnahme der Stelle aus Aufnahme A. Maßstabsbalken: A/B/D: $10 \mu \mathrm{m}, \mathrm{C}: 500 \mathrm{~nm}$. E Histogrammanalyse der Höhenverteilung im rot umrahmten Bereich von Aufnahme B. F Höhenprofil entlang der schwarzen Linie in Aufnahme C. 
In Aufnahmen A-C sind deutlich zwei unterschiedliche Höhenniveaus erkennbar. Es ist zu erwarten, dass das Glykolipid C16-GL aufgrund seiner im Vergleich zum Lysinollipid längeren Alkylketten höhere Strukturen liefert. In der Tat belegt ein Vergleich von Aufnahmen A und D, die an derselben Probenstelle erstellt wurden, dass die höheren Bereiche den nicht fluoreszierenden Domänen entsprechen (Abbildungen 6.17 und 6.19). Diese Ergebnisse belegen, dass die Domänen mit dem Glykolipid C16-GL angereichert sind (vgl. Kapitel 6.2.2). Der Unterschied zwischen den beiden Höhenniveaus, die in den AFMMessungen detektiert wurden, wurde über Histogrammanalysen (Abbildung 6.20 E) zu $(0,9 \pm 0,2) \mathrm{nm}(n=5)$ bestimmt. Ein ähnliches Ergebnis liefert auch das in F dargestellte Höhenprofil. Da die Höhe einer Lysinolmonoschicht mit einer darunterliegenden DPPCSchicht in Kapitel 6.1.1 zu $(4,2 \pm 1,6) \mathrm{nm}$ bestimmt wurde, liegt die Höhe einer C16-GLMonoschicht auf DPPC demnach bei $(5,1 \pm 1,8) \mathrm{nm}$. Die topografischen Aufnahmen A/B belegen jedoch auch, dass zwar ein Teil von C16-GL in Domänen angereichert ist, die Phasenseparation und Entmischung jedoch nicht vollständig ist. In der niedrigeren Matrix um die erhöhten Domänen sind viele hohe Strukturen erkennbar. Dieses Phänomen verursacht vermutlich auch die kleinen dunklen Stellen, die in den fluoreszierenden Bereichen der Aufnahmen in Abbildung 6.19 erkennbar sind. Die AFM-Bilder zeigen weiter, dass die Randregionen der Domänen mit niedrigen Strukturen durchsetzt sind.

Somit war die Immobilisierung der Lysinollipid/C16-GL-Monoschichten insofern erfolgreich, dass die Domänenstruktur teilweise erhalten blieb, auch wenn die Entmischung nicht optimal ist. Es steht damit eine Oberfläche zur Verfügung, die neben Bereichen, auf denen durch Anwesenheit des Lysinollipids Silicapräzipitation stattfindet, auch solche aufweist, auf denen keine Reaktion ausgelöst werden sollte. Dabei sind diese Bereiche, wie es das Ziel war, als Domänen in der umgebenden Matrix verteilt.

\subsubsection{Präzipitationsreaktionen an festkörperunterstützten Lysinollipid/C16-GL-Membranen}

Die Membranen, deren Präparation und Charakterisierung in den vorausgehenden Kapiteln dokumentiert wurde, wurden im nächsten Schritt mit monomerer Kieselsäure versetzt und anschließend rasterkraftmikroskopisch untersucht, um entstandene Silicastrukturen zu detektieren. Die hierbei erzielten Ergebnisse waren heterogen und geben kein einheitliches Bild wieder. Zwei Beispiele sind in Abbildung 6.21 aufgeführt, wobei Aufnahmen A-C von einer Probe stammen, D-F dagegen von einer anderen. In beiden Fällen zeigt ein 
Vergleich der Fluoreszenz der Oberfläche (C bzw. E) mit deren Topographie (A bzw. D) an jeweils gleichen Stellen, dass die nicht fluoreszierenden, dunklen Bereiche höher sind.
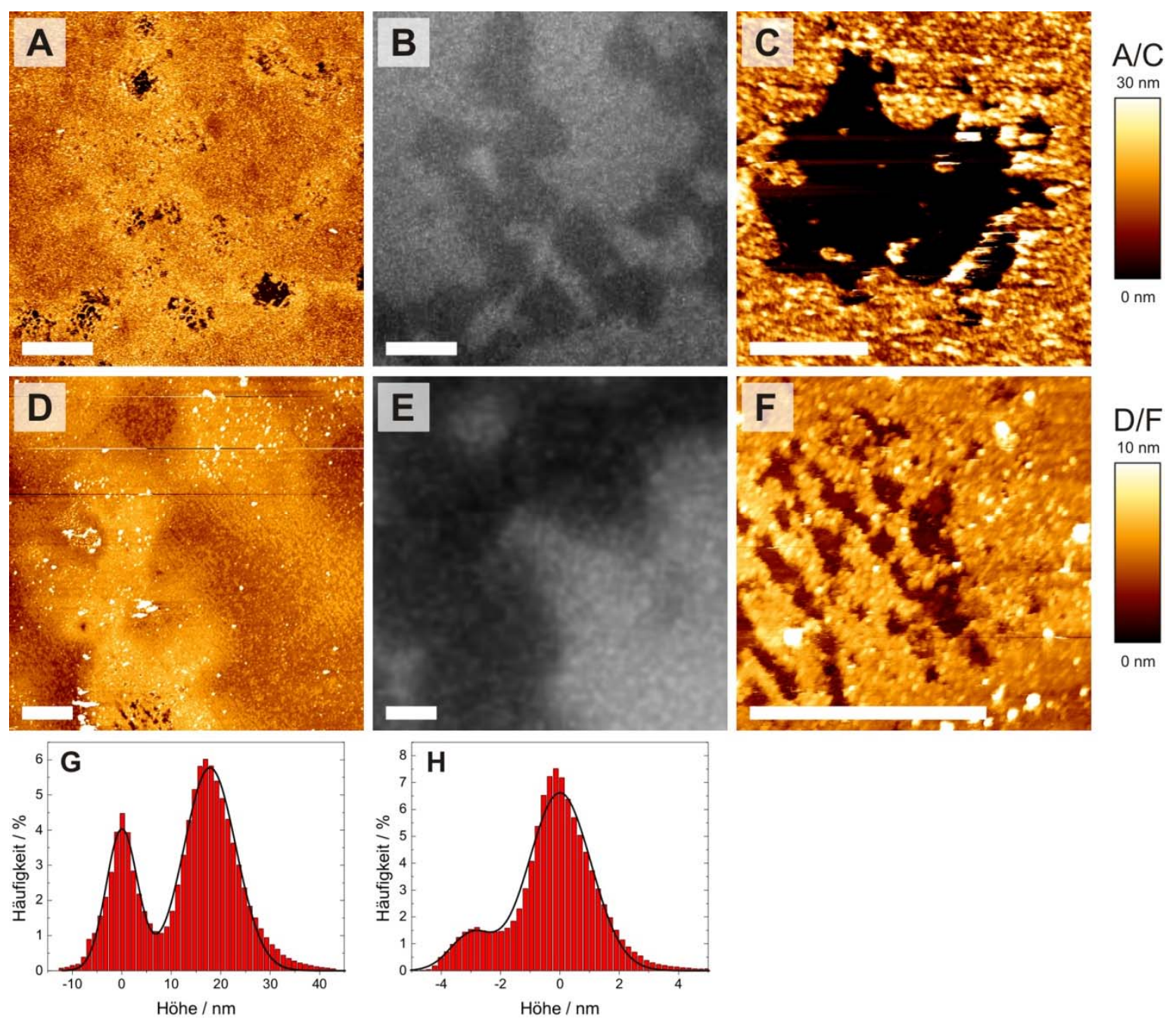

Abbildung 6.21 A/C/D/F Rasterkraftmikroskopische Aufnahmen von Lysinol/C16-GL-Lipidschichten (immobilisiert auf DPPC/Glimmer mittels LB-Übertrag bei $\pi=27 \mathrm{mN} / \mathrm{m}$ ) nach A/C einstündiger Inkubation mit $100 \mathrm{mM} \mathrm{Si}(\mathrm{OH})_{4}$ beziehungsweise $\mathrm{D} / \mathrm{F}$ zweimaliger Inkubation mit $\mathrm{Si}(\mathrm{OH})_{4}(20 \mathrm{~min} 25 \mathrm{mM}, 30 \mathrm{~min}$ $75 \mathrm{mM}$ ) jeweils in $50 \mathrm{mM}$ MES pH 6,0. Die AFM-Messungen (NWII) wurden im tapping mode mit CSC37cantilevern in Reinstwasser durchgeführt. B/E Fluoreszenzbilder der Stellen aus A bzw. D. Maßstabsbalken: A/B: $20 \mu \mathrm{m}, \mathrm{C}-\mathrm{F}: 5 \mu \mathrm{m}$. G Histogrammanalyse der Verteilung der Höhenniveaus in Aufnahme C. H Histogrammanalyse der Höhen in Aufnahme F.

Dies kann darauf zurückgeführt werden, dass in den dunklen Domänen C16-GL angereichert ist, das größere Höhen liefert ( Abbildung 6.20). Es ist jedoch auch deutlich sichtbar, dass die Probenoberfläche im Gegensatz zu der vor der Kieselsäurezugabe verändert ist. Waren die Höhenniveaus zuvor klar definiert und begrenzt (Abbildung 6.20), sind jetzt fließende Übergänge erkennbar. Außerdem zeigen höher aufgelöste Aufnahmen (Abbildung 6.21 C/F), dass die Probenoberfläche mit kugelförmigen Silicapartikeln bedeckt ist. Es wurde erwartet, dass auf den mit C16-GL angereicherten Domänen keine 
Silicabildung stattfinden sollte und somit an diesen Stellen die topografischen Aufnahmen niedrigere Bereiche anzeigen sollten. Teilweise ist dies in Aufnahmen $\mathrm{A}$ und $\mathrm{C}$ beobachtbar. Über dem Zentrum einiger Domänen sind ,Löcher' erkennbar, deren Tiefe über Histogrammanalysen $(\mathrm{G}) \mathrm{zu}(16,3 \pm 1,6) \mathrm{nm}(n=7)$ bestimmt wurde. Die Form dieser Löcher ist ungleichmäßig, die Ränder sind zerfranst. Es wurde festgestellt, dass durch mehrmaliges Rastern der Probe mit dem cantilever das Ausmaß der Löcher vergrößert wurde, also wahrscheinlich Material abgetragen wurde. Ein etwas anderes Bild ergeben Aufnahmen D und F. Hier gibt es kaum Löcher und die Tiefe des einzig beobachtbaren beträgt 3,0 nm (Histogramm H), womit es um ein Vielfaches weniger tief ist, als die zuvor beschriebenen. Zusammenfassend lässt sich feststellen, dass immer Silicapräzipitation auf den Proben stattfand und diese mehr oder weniger vollständig mit Silica bedeckt wurden. Nicht immer und wenn dann nur stellenweise wurde über den mit Glykolipid angereicherten Membrandomänen, wie dies geplant war, kein Silica detektiert.

\subsection{Diskussion}

Die biomimetische Synthese von Silicastrukturen stellt eine für viele Bereiche interessante Aufgabenstellung dar. Neben den in den vorhergehenden Kapiteln erwähnten Reaktionen, die in Lösungen durchgeführt werden, gibt es Bemühungen, auch auf planaren Substraten beziehungsweise an planaren Grenzflächen unter milden Bedingungen mittels Biomineralisation $\mathrm{SiO}_{2}$-Strukturen $\mathrm{zu}$ synthetisieren. Dabei kamen zum Beispiel Polyamine oder Polypeptide zum Einsatz, die auf Oberflächen immobilisiert wurden und dort Polymerisationsreaktionen auslösen. Die Polymere wurden dabei beispielsweise durch surface-grafting ${ }^{184}$ synthetisiert oder durch elektrostatische Wechselwirkungen immobilisiert. ${ }^{133}$ Bei Inkubation mit Kieselsäure wird dabei ein Silicafilm gebildet. Um definiert strukturiertes Silica bilden zu können, kamen Techniken wie photochemisches grafting, ${ }^{152,185}$ holografische zweiphotonen-induzierte Photopolymerisation, ${ }^{186}$ Photolithografie $^{187}$ oder ink writing $^{155}$ zum Einsatz, mit derer Hilfe die reaktiven Verbindungen zu Templaten vorstrukturiert wurden. In einem weiteren Ansatz wurde das R5-Peptid $\left(\mathrm{H}_{2} \mathrm{~N}-\mathrm{SSKKSGSYSGSKGSKRRIL-CO}{ }_{2} \mathrm{H}\right)$, ein polykationisches Vorstufenpeptid von Silaffin-1A1 ohne posttranslationale Modifikationen (vgl. Abbildung 1.7 A), an eine Lipidmembran an der Wasser-Luft-Grenzfläche gebunden. ${ }^{188}$ Reaktionen, die direkt durch funktionelle Kopfgruppen von Lipiden induziert wurden, sind bisher jedoch nur mit mizellaren und vesikulären Systemen bekannt (vgl. Kapitel 5). In der vorliegenden Arbeit 
wurde eines der in Kapitel 5 vorgestellten Aminolipide eingesetzt, um in einer schnellen biomimetischen Reaktion planare Silicastrukturen zu synthetisieren. Zu diesem Zweck wurden auf zwei unterschiedliche Arten festkörperunterstützte Doppelschichten des Lysinollipids präpariert und charakterisiert, bevor über mehrere Methoden erfolgreich Silicapräzipitationen an der Oberfläche nachgewiesen werden konnten. Zudem wurde ein Ansatz verfolgt, durch den definierte Strukturen in den Silicafilmen erzeugt werden sollten. Dazu wurde ein binäres Lipidsystem verwendet, in welchem durch Selbstorganisation Domänen gebildet wurden, die als Template für die Präzipitation dienen sollten.

\section{Präparation der Lysinollipiddoppelschichten}

Die Präparation der Lysinollipiddoppelschichten wurde sowohl durch Spreiten von Vesikeln als auch durch Langmuir-Blodgett(LB)-Übertrag umgesetzt. Der Prozess des Vesikelspreitens wurde durch ellipsometrische Messungen (Abbildung 3.1) verfolgt, wobei anhand der Änderung der Winkel eine qualitative Zunahme der Schichtdicke beobachtet wurde, jedoch an dieser Stelle die Schichtdicke nicht bestimmt werden konnte (Abbildung 6.1). Bei der anschließenden Charakterisierung der Proben mittels Fluoreszenz- und Rasterkraftmikroskopie wurden homogen leuchtende, glatte Oberflächen ohne Defekte beobachtet (Abbildungen 6.2 und 6.3). Andere Arbeiten mit positiv geladenen Lipiden deuten darauf hin, dass bei den Spreitprozessen Doppelschichten entstehen. ${ }^{168,169,189-191} \mathrm{Um}$ eindeutig zu klären, ob es sich bei der planaren Lipidschicht um eine Doppel- oder eine Multischicht handelt, wurde die Schichtdicke anhand ellipsometrischer Daten unter Verwendung eines Modells für die übereinanderliegenden Schichten mit festgelegten Brechungsindizes (siehe Abschnitt 9.1) im Rahmen einer Diplomarbeit ${ }^{192}$ bestimmt. Es ergab sich eine Schichtdicke von $(3,4 \pm 0,3) \mathrm{nm}(n=3)$. Diese Höhe lässt darauf schließen, dass eine Lipiddoppelschicht ausgebildet wurde. Die Lysinollipidmonoschichten, die mittels LB-Technik auf mit DPPC beschichteten Siliciumwafern immobilisiert wurden, weisen mit $(4,2 \pm 1,6) \mathrm{nm}(n=4)$ (ermittelt über AFM-Messungen, Abbildung 6.6) eine etwas größere Höhe auf. Die Differenz lässt sich dadurch erklären, dass die Alkylketten von DPPC um jeweils vier Methyleneinheiten länger sind als diejenigen des Lysinollipids (vgl. Abbildung 6.22). Die Differenz zwischen den theoretisch bestimmten Moleküllängen (DPPC: ca. $3 \mathrm{~nm}$, Lysinollipid: ca. $2,5 \mathrm{~nm}$, bestimmt mit ChemBio 3D Ultra) beträgt ca. 0,5 nm, was sich mit den Beobachtungen gut deckt. Die mittels LB-Technik immobilisierten Membranen wiesen eine große Zahl von Defekten auf, die sowohl in 
Fluoreszenz- als auch in AFM-Aufnahmen sichtbar waren (Abbildungen 6.5 und 6.6). Die Defekte werden wahrscheinlich durch elektrostatische Repulsion zwischen den positiv geladenen Kopfgruppen bedingt, die beim Übertrag eine nicht ideale Immobilisierung verursachen. Ein weiterer Grund ist der mit $\pi=27 \mathrm{mN} / \mathrm{m}$ relativ geringe Oberflächendruck, der für den Übertrag gewählt wurde. Da Lysinollipidmonoschichten große Substanzverluste in die Subphase erleiden und dieses Phänomen mit steigendem Oberflächendrücken noch verstärkt wird, konnte jedoch kein höherer Druck angewendet werden.

\section{Silicapräzipitation an planaren Lysinollipidschichten}

Die Inkubation festkörperunterstützter Lysinollipidschichten mit Kieselsäure bewirkte in allen Fällen einen Schichtdickenzuwachs und eine Erhöhung der Rauheit der Oberfläche. Alle Ergebnisse sprechen für eine an der Grenzfläche ablaufende Polymerisation, bei der Silica abgelagert wird. Auf den Oberflächen waren in AFM- sowie SEM-Aufnahmen sowohl in wässrigem Medium als auch nach Trocknen an Luft kugelförmige Silicastrukturen erkennbar (Abbildungen 6.9, 6.11, 6.13 und 6.14).

Die ellipsometrischen Untersuchungen zeigten, dass die Reaktionen unmittelbar nach Zugabe der Kieselsäure einsetzen und bereits nach nur zehn bis zwanzig Minuten größtenteils beendet waren (Abbildung 6.7). Danach änderte sich die Schichtdicke zwar weiter konstant, jedoch nur noch in sehr geringem Ausmaß. Es wird davon ausgegangen, dass zwei Prozesse überlagert sind, zum einen die Präzipitationsreaktion und zum anderen eine Sol-Gel-Reaktion. Die Prozesse, die dabei an durch Spreiten von Vesikeln präparierten Lysinollipidmembranen stattfinden, wurden mittels Ellipsometrie im Rahmen einer Diplomarbeit ${ }^{192}$ weiter analysiert. An den Verlauf der Kurve wurde eine exponentielle Funktion zweiten Grades angepasst, wodurch zwei Geschwindigkeitskonstanten ermittelt werden konnten. Für die erste Reaktion ergab sich $k_{l}=(22 \pm 5) \cdot 10^{-3} \mathrm{~s}^{-1}$, für die zweite $k_{2}=(23 \pm 3) \cdot 10^{-4} \mathrm{~s}^{-1}$. Diese Werte belegen die Aussage, dass zwei Reaktionsprozesse stattfinden, von denen der erste, die Präzipitationsreaktion, um das Zehnfache schneller ist, als der zweite, der einer Sol-Gel-Bildung entspricht. Die Präzipitation wird durch die Aminogruppen induziert, während der Sol-Gel-Prozess unabhängig von den Prozessen an der Oberfläche stattfindet, wie das in instabilen Silicasolen der Fall ist. Der Sol-GelProzess kann durch den Überschuss an Kieselsäure auch nach Beendigung der Präzipitation und damit der Bedeckung der positiv geladenen Oberfläche mit Silica weiter stattfinden, da er keiner Interaktion mit den Aminogruppen bedarf. 
In der von J. Ziomkowska durchgeführten Diplomarbeit ${ }^{192}$ konnte weiterhin die Schichtdicke der Silicaablagerungen auf der Oberfläche, durch Verwendung eines Schichtmodells mit festgelegten Brechungsindizes (siehe Abschnitt 9.2) zu (1,6 $\pm 0,2) \mathrm{nm}$ bestimmt werden. Da jedoch für die Silicaschicht eine planare, glatte Oberfläche angenommen wurde und nicht, wie die AFM-Aufnahmen zeigen (Abbildung 6.9), eine mit runden Silicapartikeln belegte, unebene Oberfläche, ist dieser Wert unterbestimmt. Bei Berechnung mittels ,Effektiv-Medien-Theorie‘ (Bruggeman-Modell) wird der tatsächliche Zustand der Oberflächenstruktur treffender beschrieben. Für einen ähnlichen Prozess (Silicapräzipitation auf einer festkörperunterstützten Lipidschicht des quartären Aminolipids) ergab sich dabei unter Annahme eines Volumenverhältnisses Silica/Puffer $=60 / 40$ ein um den Faktor 2 größere Schichtdicke, als bei Auswertung mit dem herkömmlichen Modell für planare Schichten. Daraus kann geschlossen werden, dass auch im Fall des Lysinollipids die Silicaschichtdicke größer als 1,6 nm ist. Wird ein Faktor von zwei angenommen, ergibt sich eine Schichtdicke von 3,2 nm, die damit genau in dem Bereich liegt, der mittels rasterkraftmikroskopischer Untersuchung ermittelt wurde. Für mittels LBTechnik präparierte Lysinollipidschichten betrugen die darauf abgelagerten Silicaschichtdicken (3 - 4) nm (Abbildung 6.11).

Es kann festgehalten werden, dass die Silicaschichtdicken unabhängig von der Präparation der planaren Lipidschichten in einer Größenordnung von unter $10 \mathrm{~nm}$ liegen und dass sich die Schichten aus runden Silicapartikeln zusammensetzen. Im Fall der mittels LB-Übertrag präparierten Membranen finden sich auch in den Defektstellen Silicakugeln. Diese Erkenntnisse sind in Abbildung 6.22 schematisch dargestellt. 

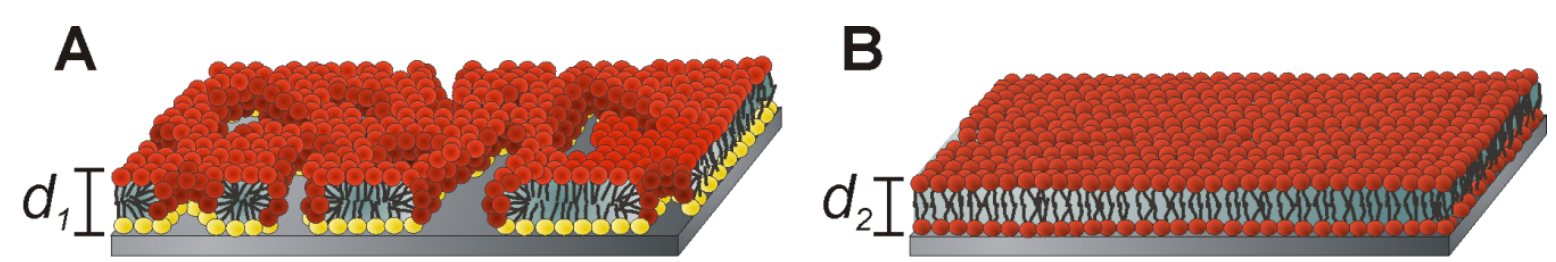

\section{$+\mathrm{Si}(\mathrm{OH})_{4}$}
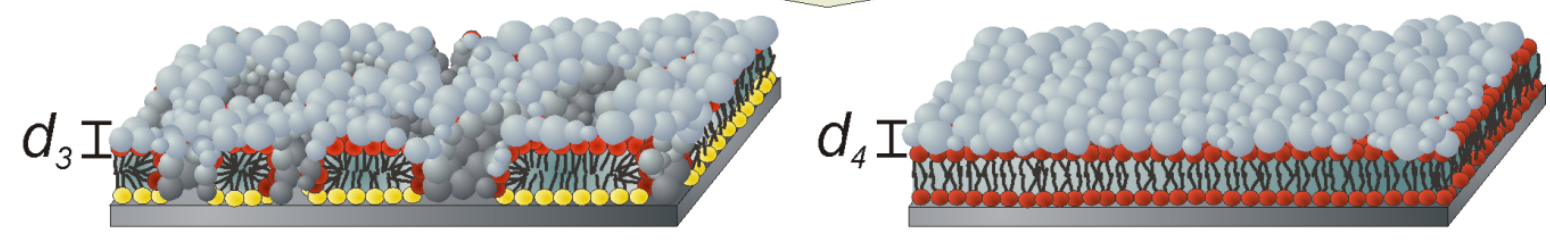

$$
\left\{\begin{array}{llll}
\text { Lysinollipid }\{\text { DPPC OSilica } & d_{1}=4,2 \mathrm{~nm} & d_{3}=3-4 \mathrm{~nm} \\
d_{2}=3,4 \mathrm{~nm} & d_{4}=1,6 \mathrm{~nm}
\end{array}\right.
$$

Abbildung 6.22 Modell der Silicapräzipitation auf festkörperunterstützten Lysinollipidschichten, die A durch LB-Übertrag beziehungsweise B durch Spreiten von Vesikeln präpariert wurden. Je dunkler Objekte einer Art eingefärbt sind, desto tiefer sind sie gelegen. $d_{l}$ : Höhe einer Lysinollipid- auf einer DPPCMonoschicht, $d_{2}$ : Höhe einer Lysinollipiddoppelschicht, $d_{3} / d_{4}$ : jeweilige Höhe der Silicaschicht. $d_{1}$ sowie $d_{3}$ wurden mittels AFM-Messungen ermittelt. $d_{2}$ und $d_{4}$ ergaben sich aus Ellipsometriemessungen.

Der Vergleich von hochaufgelösten Bildern der Silicazellwände von Diatomeen mit den vermessenen Oberflächen mit den kugelförmigen Strukturen deckt einige Ähnlichkeiten auf. Rasterkraftmikroskopische Untersuchungen sowohl an lebenden Diatomeen ${ }^{193-195}$ als auch an Präparaten ihrer Zellwände ${ }^{195-200}$ zeigten, dass deren Oberfläche unter einer organischen Schicht nicht planar, sondern aus kleinen Kugeln zusammengesetzt ist, deren Größe zu $(40-45) \mathrm{nm}^{201}$ beziehungsweise $(50-80) \mathrm{nm}^{202}$ bestimmt wurde. Diese Silicakugeln sind auch in elektronenmikroskopischen Aufnahmen beobachtet worden. ${ }^{203}$ Abbildung 6.23 gibt Beispiele von AFM-Aufnahmen wieder. Die Topografien erinnern teilweise an diejenigen in Abbildung 6.9 beziehungsweise Abbildung 6.11. Die Entstehung der sphärischen Silicastrukturen an den Lysinollipidschichten lässt sich erklären, wenn davon ausgegangen wird, dass an der Oberfläche an bestimmten Punkten Keimbildung stattfindet, von denen aus radial Silica gebildet wird. Erreicht das Silica eine gewisse Schichtdicke, kann keine Kieselsäure mehr durch die Schicht diffundieren, womit die Reaktion mit den Aminogruppen zum Erliegen kommt. Das erklärt, warum die Silicaschicht nur wenige Nanometer dick ist. Überschüssige Kieselsäure lagert sich weiter in einem Sol-Gel-Prozess ab. 

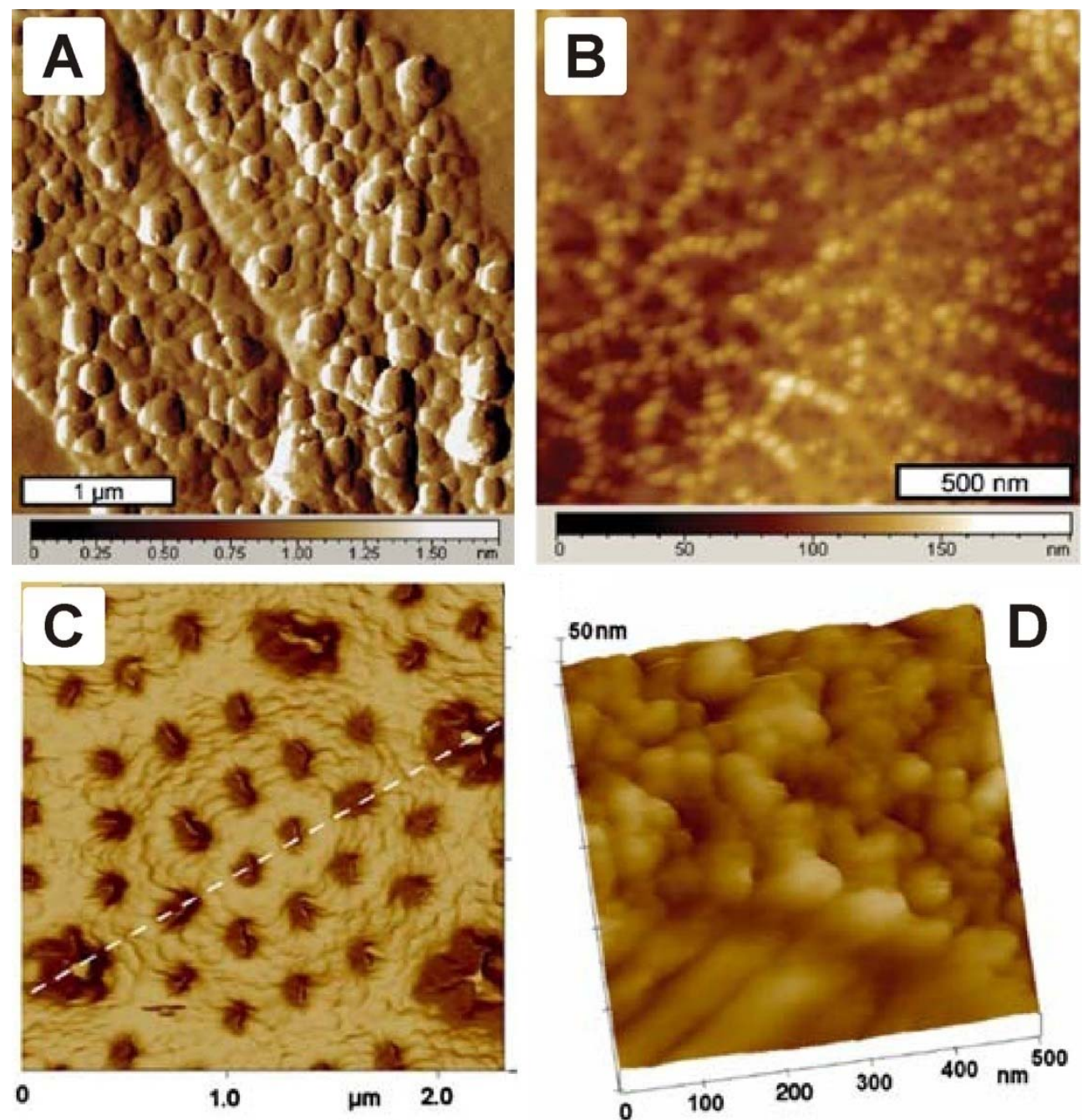

Abbildung 6.23 AFM-Aufnahmen von Silicazellwänden verschiedener Diatomeen: A Distale Oberfläche des Gürtelbands von Skeletonema costatum ${ }^{200}$, B distale Oberfläche der Valva von Thalassiosira pseudonana ${ }^{200}$, C Cribrum von Coscinodiscus sp. ${ }^{198}$ sowie D distale Oberfläche des Frustulums von Thalassiosira eccentrica ${ }^{198}$.

Zusammenfassend kann festgehalten werden, dass durch biomimetische Präzipitation an Oberflächen von immobilisierten Amphiphilen, die wie Silaffine und Polyamine Aminogruppen enthalten, auf kurzer Zeitskala unter milden Bedingungen Silicastrukturen erzeugt werden konnten, die den in Diatomeen auftretenden sehr ähnlich sind. Die dabei gebildete Silicaschicht ist wenige Nanometer dick und fest mit der Membran verbunden. Ein interessanter Aspekt ist hierbei, dass die Anwesenheit von anionischen Spezies in der positiv geladenen Oberfläche nicht nötig ist, was in anderen Arbeiten postuliert wurde. ${ }^{204}$

\section{Binäre Lysinollipid/C16-GL(4/1)-Mischungen}

Das Verhalten der binären Monoschichten, die Lysinollipid und C16-GL beinhalten, und ihre Immobilisierung wurde in Kapiteln 6.2.2 sowie 6.2.3 eingehend beschrieben und diskutiert. Es konnten phasenseparierte Lipidmonoschichten erzeugt werden, in denen 
C16-GL in Domänen angereichert ist. Diese Monoschichten können mittels LB-Technik auf Substraten immobilisiert werden, wobei jedoch die Domänenstruktur nur teilweise erhalten bleibt und die Lipide nicht vollständig entmischt sind (Abbildung 6.20).

\section{Silicapräzipitation an immobilisierten Lysinollipid/C16-GL(4/1)- Schichten}

Bei der Präzipitation auf Lipidschichten bestehend aus Lysinollipid und dem Glykolipid C16-GL wurde erwartet, dass in Bereichen, in denen Lysinollipid vorhanden ist, Silica entsteht, während im Bereich der C16-GL-Domänen die Silicabildung ausbleiben sollte, um auf diese Weise eine Silicastruktur mit definierten Poren zu erzeugen. Nach der Zugabe von monomerer Kieselsäure zu Lysinollipid/C16-GL(4/1)-Schichten wurden, wie bei reinen Lysinollipidschichten, runde Silicapartikel auf der Oberfläche detektiert (Abbildung 6.21). Dies spricht dafür, dass die Silicabildung grundsätzlich wie auf den Lysinollipidschichten erfolgt. Es waren teilweise Bereiche mit unterschiedlichen Höhenniveaus beobachtbar, die, in Korrespondenz mit den Ergebnissen aus Abbildung 6.21, schematisch in Abbildung 6.24 wiedergegeben sind. Zum einen traten erhöhte Bereiche auf, in denen Silica über den C16-GL-Domänen abgelagert ist (Abbildung 6.24 A) zum anderen wurden an Stellen, an denen C16-GL-Domänen in der Membran vorhanden waren, niedriger liegenden Bereiche detektiert. Hierbei müssen zwei verschiedene Fälle unterschieden werden: Einerseits Bereiche mit einer Tiefe von ca. $3 \mathrm{~nm}$ (Abbildung 6.21 F) und andererseits solche mit einer Tiefe von 16,3 nm (Abbildung 6.21 C). Wichtig ist an dieser Stelle, dass das Ausmaß der Bereiche mit 16,3 nm Tiefe durch mehrmaliges Abtasten mit der AFM-Messspitze vergrößert wurde. Eine Erklärung für diese Beobachtungen ist, dass im Fall der $3 \mathrm{~nm}$ tiefen Löcher kein Silica gebildet wurde (Abbildung 6.24 B), wie dies auch erwartet wurde, da G16-GL keine Silica-präzipitierende Funktion besitzen sollte. Die Höhe von $3 \mathrm{~nm}$ stimmt mit der Höhe der Silicaschichten, die auf Lysinollipidschichten detektiert wurden, nämlich (3-4) nm, überein. Die tieferen Löcher entstanden wahrscheinlich durch Abtragung von sowohl Silica als auch darunterliegenden Lipiden während der AFM-Messung (Abbildung 6.24 C). Die Höhendifferenz von 16,3 $\mathrm{nm}$ setzt sich in diesem Fall aus der Höhe der beiden Lipidmonoschichten $(5,1 \mathrm{~nm})$ und darüber liegendem Silica (ca. $11 \mathrm{~nm}$ ) zusammen. Die Silicaschicht ist in diesem Fall dicker, liegt aber immer noch im gleichen Größenbereich. 


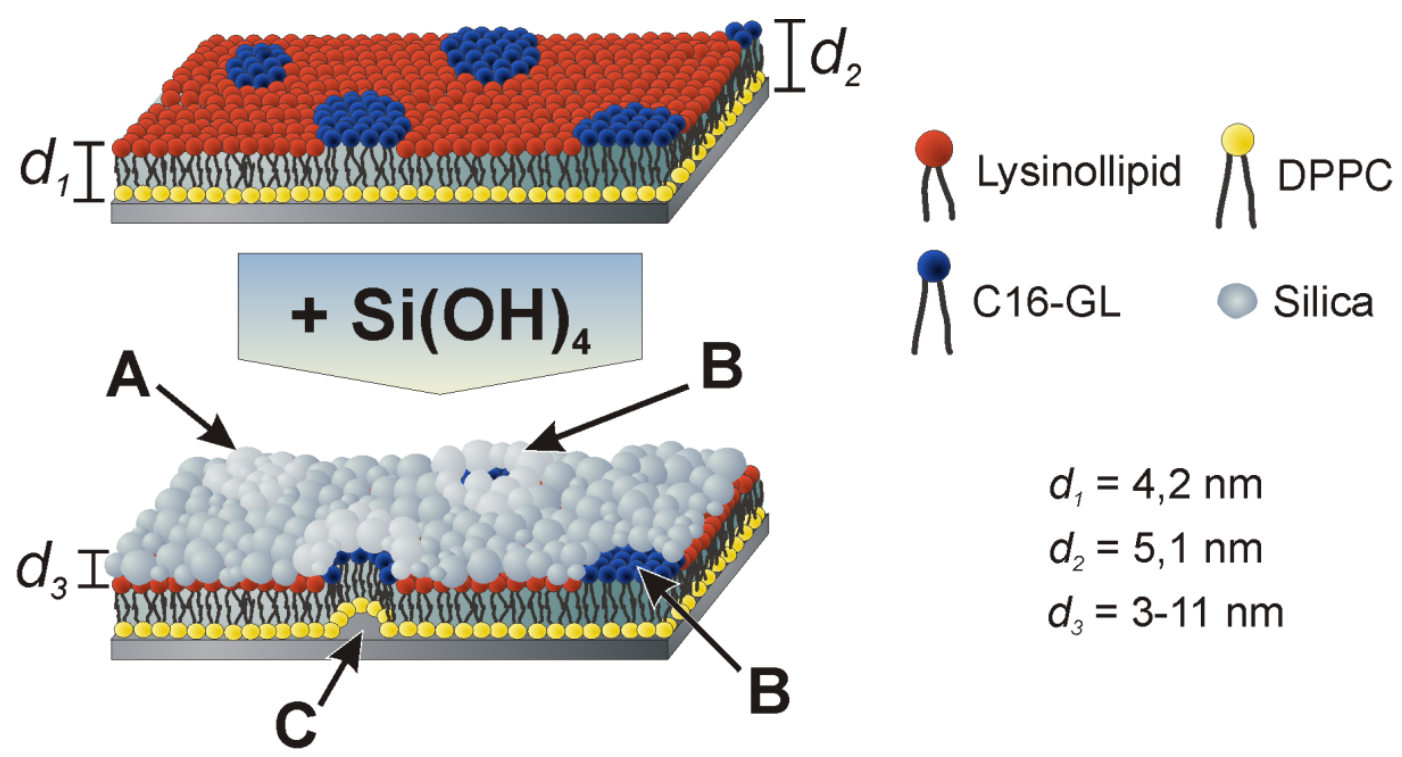

Abbildung 6.24 Schematische Abbildung der Silicapräzipitation auf immobilisierten Lysinollipid/C16-GLSchichten. Es sind drei mögliche Ergebnisse dargestellt: A Die C16-GL-Domänen sind mit Silica bedeckt, wobei die Domänen an der erhöhten Topografie des Silica erkennbar sind. B Die C16-GL-Domänen sind nicht/nur teilweise mit Silica bedeckt. C Durch das Abrastern der Probe mit dem cantilever wird bei AFMMessungen Silica zusammen mit den darunterliegenden Lipiden abgetragen. $d_{l}$ : Höhe der Lysinollipid- auf einer DPPC-Monoschicht, $d_{2}$ : Höhe des in Domänen angereicherten C16-GL auf einer DPPC-Monoschicht, $d_{3}$ : Schichtdicke des präzipitierten Silica. $d_{1}-d_{3}$ wurden mittels rasterkraftmikroskopischen Messungen bestimmt.

Es muss an dieser Stelle geklärt werden, warum auch im Bereich der C16-GL-Domänen Silica präzipitiert wurde. Nicht vollständig geklärt werden kann im Rahmen dieser Arbeit, ob C16-GL integriert in planare Membransysteme möglicherweise mit Kieselsäure interagiert und dies die weitestgehend vollständig Belegung der Oberflächen mit Silicapartikeln verursacht. Im Fall C16-GL-Vesikeln, also sphärischen Membransystemen mit gekrümmten Oberflächen konnte eine solche Wechselwirkung jedoch ausgeschlossen werden (siehe Abschnitt 6.2.1).

Eine andere mögliche Ursache stellt die nicht ausreichende Phasenseparation der beiden Lipide dar, aufgrund derer sich Lysinollipidmoleküle auch in den C16-GL-Domänen befanden. Dies ist in den AFM-Aufnahmen der Lipidschichten (Abbildung 6.20) deutlich erkennbar. Es muss davon ausgegangen werden, dass die unvollständige Entmischung der beiden Komponente eine klar definierte Trennung von reaktiver und nicht-reaktiver Oberfläche verhindert. Aus diesem Grund wurde nur teilweise die Domänenstruktur der Lipidschicht in der Silicastruktur abgebildet (Abbildung 6.21). Dieses Problem könnte dadurch gelöst werden, dass die Lipiddoppelschichten mittels einer anderen Methode präpariert werden, womit unter Umständen eine bessere Phasenseparation erzielt werden kann. Erste Versuche, bei denen Vesikel einer Mischung von Lysinollipid und C16-GL auf 
Siliciumwafer gespreitet wurden zeigten dahin gehend Erfolge und bieten einen vielversprechenden Ansatz für weitere Experimente.

Abschließend lässt sich zusammenfassen, dass die planaren Lipidschichten die Bildung von planaren Silicastrukturen ermöglichen. Die Bildung von Silicaschichten mit einer definierten Porenstruktur gelang ansatzweise (Abbildung 6.21), womit die Grundlage für weitere Untersuchungen und für eine Weiterentwicklung der Membransysteme als Template für biomimetische Silicapräzipitationen geschaffen wurde. 


\section{Zusammenfassung}

Langkettigen Polyaminen wird eine entscheidende Rolle bei der Bildung der hierarchisch strukturierten Silicazellwände von Diatomeen zugesprochen. Im Rahmen der vorliegenden Arbeit wurden Polyamine genau definierter Strukturen untersucht, die in ihren Kettenlängen den in Diatomeen vorkommenden entsprechen. Im Vordergrund stand der Einfluss der Strukturmerkmale Kettenlänge, Alkylspacer und $N$-Methylierung auf die Silicapräzipitation. Zunächst wurde gezeigt, dass die Polyamine in Anwesenheit von Phosphationen die Polymerisation von Silica aus monomerer Kieselsäure induzieren, wobei innerhalb von zehn Minuten hohle Silicaskugeln mit Schalendicken von 20-60 nm und Durchmessern von 50-160 nm gebildet werden, die für jede Verbindung innerhalb einer schmalen Verteilung liegen. Weiterhin wurde nachgewiesen, dass die Variation der Strukturmerkmale das entstehende Silica spezifisch beeinflusst. So nehmen sowohl Silicamenge als auch Partikelgröße mit steigender Kettenlänge, Länge der Alkylgruppe sowie $\mathrm{N}$-Methylierung $\mathrm{zu}$ und auch die Silicamorphologie wird durch die genannten Faktoren bestimmt. Theoretische Betrachtungen des Säure-Base-Verhaltens der Moleküle legten offen, dass diese Beobachtungen über ein Zusammenspiel von Protonierung und Hydrophobizität der Polyamine erklärt werden können. Der Grund hierfür ist, dass diese Parameter Einfluss auf die Phasenseparation und die Vernetzung der Polyamine untereinander haben.

Die Etablierung von selbstorganisierenden Templatsystemen für Silicapräzipitationen gelang durch den Einsatz von synthetischen Aminoverbindungen mit hydrophoben Membranankern, den sogenannten Aminolipiden. Es konnten erfolgreich Vesikel und planare Lipidschichten dieser Verbindungen aufgebaut werden, an deren Oberflächen nach Zugabe monomerer Kieselsäure innerhalb kurzer Zeit Silicabildung qualitativ und quantitativ nachgewiesen wurde.

Im Fall der Aminolipide wird, anders als bei den Polyaminen, die Aggregation durch die hydrophoben Interaktionen der Alkylketten der Amphiphile gesteuert. Die Tatsache, dass an den Grenzflächen dieser Aggregate in Abwesenheit von Phosphationen Silicapräzipitationen stattfinden, gibt einen klaren Hinweis darauf, dass bei der biomimetischen Bildung von Silica entscheidend ist, dass eine Phasenseparation stattfindet und damit einhergehend positive Ladungen an Grenzflächen akkumuliert werden.

Die Verwendung von Aminolipidvesikeln als Reaktionstemplate erlaubte neben der Bildung sphärischer Silicapartikel die Untersuchung des Einflusses der $N$-Methylierung 
der Aminogruppe auf die Präzipitationsreaktion, die einen direkten Zusammenhang zwischen Ladung der Oberfläche und entstehender Silicamenge aufzeigte. Weiter konnte gezeigt werden, dass auch an der Oberfläche von planaren Doppelschichten, bestehend aus einem Aminolipid, eine schnelle biomimetische Präzipitation stattfindet. Es entsteht eine aus Silicakugeln aufgebaute Schicht mit einer Höhe von wenigen Nanometern, die eine Feinstruktur ähnlich der der Zellwände von Diatomeen besitzt. Letztendlich wurde der Grundstein für die Bildung strukturierter planarer Silicastrukturen gelegt, indem die selbstorganisierende Domänenbildung in einem binären Lipidsystem von Lysinollipid und einem Glykolipid genutzt wurde. 


\section{Literaturverzeichnis}

1 Lowenstam, H. A. Minerals Formed by Organisms, Science 1981, 211(4487):11261131.

2 On biomineralization (Eds.: Lowenstam, H. A. und S. Weiner), Oxford University Press, Oxford, 1989

3 Kröger, N. und N. Poulsen Diatoms-From Cell Wall Biogenesis to Nanotechnology, Annual Review of Genetics 2008, 42:83-107.

4 Peytcheva, A. und M. Antonietti "Carving on the nanoscale": Polymers for the site-specific dissolution of calcium phosphate, Angew. Chem., Int. Ed. 2001, 40(18):3380-3383.

5 Biomineralization: From Biology to Biotechnology and Medical Application (Ed.: Baeuerlein, E.), Wiley-VCH, Weinheim, 2000

6 Tacke, R. Milestones in the Biochemistry of Silicon: From Basic Research to Biotechnological Applications, Angew. Chem., Int. Ed. 1999, 38(20):3015-3018.

7 Treguer, P., D. M. Nelson, A. J. Vanbennekom, D. J. Demaster, A. Leynaert und B. Queguiner The Silica Balance in the World Ocean - a Reestimate, Science 1995, 268(5209):375-379.

$8 \quad$ Silicon and Siliceous Structures in Biological Systems (Eds.: Simpson, T. L. und B. E. Volcani), Springer, New York, 1981

9 Kooistra, W. H., M. De Stefano, D. G. Mann und L. K. Medlin in Silicon Biomineralization, Vol. 33 (Ed.: W. E. G. Müller), Springer: Berlin, 2003, pp. 5997.

10 Baeuerlein, E. Biomineralisation von Einzellern: eine außergewöhnliche Membranbiochemie zur Produktion anorganischer Nano- und Mikrostrukturen, Angew. Chem. 2003, 115:636-664.

11 Mann, D. G. und S. J. M. Droop Biodiversity, biogeography and conservation of diatoms, Hydrobiologia 1996, 336(1-3):19-32.

12 Werner, D. in Biology of Diatoms, Botanical Monographs, Vol. 13 (Ed.: D. Werner), Blackwell Scientific Publications: Oxford, 1977, pp. 1-17.

13 Raven, J. A. The Transport and Function of Silicon in Plants, Biol. Rev. 1983, 58(2):179-207.

14 Smetacek, V. Diatoms and the ocean carbon cycle, Protist 1999, 150(1):25-32. 
15 Hamm, C. E., R. Merkel, O. Springer, P. Jurkojc, C. Maier, K. Prechtel und V. Smetacek Architecture and material properties of diatom shells provide effective mechanical protection, Nature 2003, 421(6925):841-843.

16 Milligan, A. J. und F. M. M. Morel A proton buffering role for silica in diatoms, Science 2002, 297(5588):1848-1850.

17 Pickett-Heaps, J., A.-M. M. Schmid und L. A. Edgar in Progress in Phycological Research, Vol. 7 (Eds.: F. E. Round, D. J. Chapman), Biopress: Bristol, 1990, pp. 1-168.

18 Hildebrand, M. und R. Wetherbee in Silicon Biomineralization, Vol. 33 (Ed.: W. E. G. Müller), Springer: Berlin, 2003, pp. 11-57.

19 Drum, R. W. und H. S. Pankratz Post mitotic fine structure of Gomphonema parvulum, J. Ultrastruct. Res. 1964, 10:217-223.

20 Vrieling, E. G., W. W. C. Gieskes und T. P. M. Beelen Silicon deposition in diatoms: control by the $\mathrm{pH}$ inside the silicon deposition vesicle., J. Phycol. 1999, 35:548-559.

$21 \mathrm{Li}, \mathrm{C} . \mathrm{-W}$. , Chu, S., Lee, M. Characterizing the silica deposition vesicle of diatoms, Protoplasma 1989, 151:158-163.

22 Del Amo, Y. und M. A. Brzezinski The Chemical Form of Dissolved Si Taken up by Marine Diatoms, J. Phycol. 1999, 35:1162-1170.

23 Hildebrand, M., B. E. Volcani, W. Gassmann und J. I. Schroeder A gene family of silicon transporters, Nature 1997, 385(6618):688-689.

24 Hildebrand, M. in Biomineralization (Ed.: E. Baeuerlein), Wiley-VCH Verlag $\mathrm{GmbH}, 2000$, pp. 171-188.

25 Thamatrakoln, K. und M. Hildebrand Silicon uptake in diatoms revisited: A model for saturable and nonsaturable uptake kinetics and the role of silicon transporters, Plant Physiol. 2008, 146(3):1397-1407.

26 Lenoci, L. und P. J. Camp Diatom structures templated by phase-separated fluids, Langmuir 2008, 24(1):217-223.

27 Round, F., R. Crawford und D. Mann, The Diatoms, Cambridge University Press: Cambridge, 1990.

28 Robinson, D. H. und C. W. Sullivan How Do Diatoms Make Silicon Biominerals, Trends Biochem. Sci. 1987, 12(4):151-154.

29 Gordon, R. und R. W. Drum The Chemical Basis of Diatom Morphogenesis, International Review of Cytology - a Survey of Cell Biology, Vol 150 1994, 150:243-372. 
30 Parkinson, J., Y. Brechet und R. Gordon Centric diatom morphogenesis: a model based on a DLA algorithm investigating the potential role of microtubules, Biochimica Et Biophysica Acta-Molecular Cell Research 1999, 1452(1):89-102.

31 Kröger, N., S. Lorenz, E. Brunner und M. Sumper Self-assembly of highly phosphorylated silaffins and their function in biosilica morphogenesis, Science 2002, 298(5593):584-586.

32 Sumper, M. A phase separation model for the nanopatterning of diatom biosilica, Science 2002, 295(5564):2430-2433.

33 Vrieling, E. G., T. P. M. Beelen, R. A. van Santen und W. W. C. Gieskes Mesophases of (Bio)Polymer-Silica Particles Inspire a Model for Silica Biomineralization in Diatoms, Angew Chem Int Ed 2002, 41(9):1543 - 1546.

34 Kröger, N. und R. Wetherbee Pleuralins are involved in theca differentiation in the diatom Cylindrotheca fusiformis, Protist 2000, 151(3):263-273.

35 Iler, R. K., The Chemistry of Silica, Wiley: New York, 1979.

36 Kröger, N. und M. Sumper in Biomineralization, 2 ed. (Ed.: E. Baeuerlein), WileyVCH: Weinheim, 2004, pp. 137-158.

37 Hollemann, A. F., E. Wiberg und N. Wiberg, Lehrbuch der anorganischen Chemie, 91. - 100. ed., de Gruyter: New York, 1985.

38 Brinker, C. J. und G. W. Scherer, Sol-gel science : the physics and chemistry of solgel processing, Academic Press: Boston, 1990.

39 Coradin, T. und P. J. Lopez Biogenic silica patterning: simple chemistry or subtle biology?, ChemBioChem 2003, 4(4):251-259.

40 Ries, H. E. und B. L. Meyers Microelectrophoresis and Electron-Microscope Studies with Polymeric Flocculants, Journal of Applied Polymer Science 1971, 15(8):2023-2034.

41 Brunner, E., K. Lutz und M. Sumper Biomimetic synthesis of silica nanospheres depends on the aggregation and phase separation of polyamines in aqueous solution, Phys. Chem. Chem. Phys. 2004, 6:854-857.

42 Mizutani, T., H. Nagase, N. Fujiwara und H. Ogoshi Silicic acid polymerization catalyzed by amines and polyamines, Bull. Chem. Soc. Jpn. 1998, 71(8):20172022.

43 Delak, K. M. und N. Sahai Amine-catalyzed biomimetic hydrolysis and condensation of organosilicate, Chem. Mater. 2005, 17(12):3221-3227.

44 Coradin, T., O. Durupthy und J. Livage Interactions of amino-containing peptides with sodium silicate and colloidal silica: A biomimetic approach of silicification, Langmuir 2002, 18(6):2331-2336. 
45 Nakajima, T. und B. E. Volcani 3,4-dihydroxyproline: a new amino acid in diatom cell walls, Science 1969, 164(886):1400-1401.

46 Nakajima, T. und B. E. Volcani $\varepsilon$-N-trimethyl-l- $\delta$-hydroxysine phosphate and its nonphosphorylated compound in diatom cell walls., Biochem. Biophys. Res. Commun. 1970, 39(1):28-33.

47 Kröger, N., C. Bergsdorf und M. Sumper Frustulins: domain conservation in a protein family associated with diatom cell walls., European journal of biochemistry / FEBS 1996, 239(2):259-264.

48 Kröger, N., C. Bergsdorf und M. Sumper A new calcium binding glycoprotein family constitutes a major diatom cell wall component, Embo J 1994, 13(19):46764683.

49 Kröger, N., G. Lehmann, R. Rachel und M. Sumper Characterization of a 200-kDa diatom protein that is specifically associated with a silica-based substructure of the cell wall., Eur. J. Biochem. 1997, 250:99 - 105.

50 Kröger, N., R. Deutzmann und M. Sumper Polycationic peptides from diatom biosilica that direct silica nanosphere formation, Science 1999, 286(5442):11291132.

51 Davis, A. K., M. Hildebrand und B. Palenik Gene expression induced by copper stress in the diatom Thalassiosira pseudonana, Eukaryotic Cell 2006, 5(7):11571168 .

52 Davis, A. K., M. Hildebrand und B. Palenik A stress-induced protein associated with the girdle band region of the diatom Thalassiosira pseudonana (Bacillariophyta), J. Phycol. 2005, 41(3):577-589.

53 Wenzl, S., R. Hett, P. Richthammer und M. Sumper Silacidins: highly acidic phosphopeptides from diatom shells assist in silica precipitation in vitro, Angew Chem Int Ed Engl 2008, 47(9):1729-1732.

54 Kröger, N., R. Deutzmann, C. Bergsdorf und M. Sumper Species-specific polyamines from diatoms control silica morphology, Proc Natl Acad Sci U S A 2000, 97(26):14133-14138.

55 Sumper, M., E. Brunner und G. Lehmann Biomineralization in diatoms: characterization of novel polyamines associated with silica, FEBS Lett 2005, 579(17):3765-3769.

56 Sumper, M. und G. Lehmann Silica pattern formation in diatoms: species-specific polyamine biosynthesis, Chembiochem 2006, 7(9):1419-1427.

57 Sumper, M. und N. Kröger Silica formation in diatoms: the function of long-chain polyamines and silaffins, Journal of Materials Chemistry 2004, 14(14):2059-2065. 
58 Kröger, N., R. Deutzmann und M. Sumper Silica-precipitating peptides from diatoms. The chemical structure of silaffin-A from Cylindrotheca fusiformis, J Biol Chem 2001, 276(28):26066-26070.

59 Poulsen, N., M. Sumper und N. Kröger Biosilica formation in diatoms: characterization of native silaffin-2 and its role in silica morphogenesis, Proc. Natl. Acad. Sci. U.S.A. 2003, 100(21):12075-12080.

60 Poulsen, N. und N. Kröger Silica morphogenesis by alternative processing of silaffins in the diatom Thalassiosira pseudonana, J. Biol. Chem. 2004, 279(41):42993-42999.

61 Sumper, M., R. Hett, G. Lehmann und S. Wenzl A code for lysine modifications of a silica biomineralizing silaffin protein, Angew. Chem., Int. Ed. 2007, 46(44):8405-8408.

62 Sumper, M. und E. Brunner Silica biomineralization in diatoms: the model organism Thalassiosira pseudonana, Chembiochem 2008, 9(8):1187-1194.

63 Sumper, M. und E. Brunner Learning from diatoms: nature's tools for the production of nanostructured silica, Advanced Functional Materials 2006, 16(1):17-26.

64 Mizutani, T., H. Nagase und H. Ogoshi Silicic Acid Polymerization Catalyzed by Amine and Polyamines, Chem. Lett. 1998, 27(2):133-134.

65 Sumper, M., S. Lorenz und E. Brunner Biomimetic control of size in the polyaminedirected formation of silica nanospheres, Angew Chem Int Ed Engl 2003, 42(42):5192-5195.

66 Kröger, N. und N. Poulsen in Handbook of Biomineralization: Biological Aspects and Structure Formation (Ed.: E. Baeuerlein), Wiley-VCH: Weinheim, 2007, pp. $43-58$.

67 Parkinson, J. und R. Gordon Beyond micromachining: the potential of diatoms, Trends Biotechnol. 1999, 17(5):190-196.

68 Bradbury, J. Nature's Nanotechnologists: Unveiling the Secrets of Diatoms, PLoS Biol. 2004, 2(10):1512-1515.

69 Wieneke, R. Philipps-Universität Marburg (Marburg), 2009.

70 Karickhoff, S. W., L. A. Carreira und S. H. Hilal SPARC On-Line Calculator, http://ibmlc2.chem.uga.edu/sparc/.

71 Wieneke, R. Philipps-Universität Marburg (Marburg), 2005.

72 Bernecker, A. Universität Regensburg (Regensburg), 2006.

73 Müller, F. Georg-August-Universität Göttingen (Göttingen), 2007. 
74 Heitmüller, S. Georg-August Universität Göttingen (Göttingen), 2009.

75 Renou, J. P., J. B. Giziewicz, I. C. Smith und H. C. Jarrell Glycolipid membrane surface structure: orientation, conformation, and motion of a disaccharide headgroup, Biochemistry 1989, 28(4):1804-1814.

76 Hinz, H. und Sturteva.Jm Calorimetric Studies of Dilute Aqueous Suspensions of Bilayers Formed from Synthetic L-Alpha-Lecithins, J. Biol. Chem. 1972, 247(19):6071-6075.

77 http://www.avantilipids.com/.

78 Groves, J. T., N. Ulman, P. S. Cremer und S. G. Boxer Substrate-membrane interactions: Mechanisms for imposing patterns on a fluid bilayer membrane, Langmuir 1998, 14(12):3347-3350.

79 Feigenson, G. W. und J. T. Buboltz Ternary phase diagram of dipalmitoylPC/dilauroyl-PC/cholesterol: nanoscopic domain formation driven by cholesterol, Biophys. J. 2001, 80(6):2775-2788.

80 Korlach, J., P. Schwille, W. W. Webb und G. W. Feigenson Characterization of lipid bilayer phases by confocal microscopy and fluorescence correlation spectroscopy, Proc. Natl. Acad. Sci. USA 1999, 96(15):8461-8466.

81 Hope, M. J., M. B. Bally, G. Webb und P. R. Cullis Production of large unilamellar vesicles by a rapid extrusion procedure. Characterization of size distribution, trapped volume and ability to maintain a membrane potential, Biochim. Biophys. Acta, Biomembr. 1985, 812(1):55-65.

82 De Smedt, F., G. Stevens, S. De Gendt, I. Cornelissen, S. Arnauts, M. Meuris, M. M. Heyns und C. Vinckier $A$ wet chemical method for the determination of thickness of SiO2 layers below the nanometer level, J. Electrochem. Soc. 1999, 146(5):1873-1878.

83 Richter, T. Universität Hamburg (Hamburg), 2004.

84 Krumeich, F. Properties of Electrons, their Interactions with Matter and Applications in Electron Microscopy, www.microscopy.ethz.ch.

85 Raschke, T. Wechselwirkungen von Elektronen mit Festkörpern am Beispiel der REM-Technik, Praktikum zur Lehrveranstaltung Festkörperelektronik und photonik.

86 Zeiss Handbuch für die Rasterelektronenmikroskope SUPRA(VP) und ULTRA.

87 Freedman, D. und P. Diaconis On the Histogram as a Density Estimator - L2 Theory, Zeitschrift Fur Wahrscheinlichkeitstheorie Und Verwandte Gebiete 1981, 57(4):453-476. 
88 Mann, H. B. und D. R. Whitney On a Test of Whether One of 2 Random Variables Is Stochastically Larger Than the Other, Annals of Mathematical Statistics 1947, 18(1):50-60.

89 Wilcoxon, F. Individual Comparisons by Ranking Methods, Biometrics Bulletin 1945, 1(6):80-83.

90 Leon, A. Mann-Whitney $U$ Test / Wilcoxon Rank Sum Test, http://elegans.swmed.edu/ leon/stats/utest.html 2007.

91 Einstein, A. Theorie der Opaleszenz von homogenen Flüssigkeiten und Flüssigkeitsgemischen in der Nähe des kritischen Zustandes, Ann. Phys. 1910, 338(16):1275-1298.

92 Einstein, A. Über die von der molekularkinetischen Theorie der Wärme geforderte Bewegung von in ruhenden Flüssigkeiten suspendierten Teilchen, Ann. Phys. 1905, 322(8):549-560.

93 Khintchine Korrelationstheorie der stationären stochastischen Prozesse, Mathematische Annalen 1934, 109(1):604-615.

94 Wiener, N. Generalized harmonic analysis Acta Mathematica 1930, 55(1):117-258.

95 Langmuir, I. Oil Lenses on Water and the Nature of Monomolecular Expanded Films, J. Chem. Phys. 1933, 1:756-776.

96 Pockels, A. Surface Tension, Nature 1891, 43:437-439.

97 Windschiegl, B. Georg-August-Universität Göttingen (Göttingen), 2009.

98 Szilluweit, R. Georg-August-Universität Göttingen (Göttingen), 2009.

99 Menke, M. Universität Regensburg (Regensburg), 2006.

100 Azzam, R. M. A. und N. M. Bashara, Ellipsometry and polarized light, 4. impression, paperback ed., Elsevier: Amsterdam, 1999.

101 Tompkins, H. G., A user's guide to ellipsometry, Dover Publications: Mineola, N.Y., 2006.

102 Belton, D. J., S. V. Patwardhan, V. V. Annenkov, E. N. Danilovtseva und C. C. Perry From biosilicification to tailored materials: optimizing hydrophobic domains and resistance to protonation of polyamines, Proc. Natl. Acad. Sci. U.S.A. 2008, 105(16):5963-5968.

103 Belton, D. J., S. V. Patwardhan und C. C. Perry Spermine, spermidine and their analogues generate tailored silicas, J. Mater. Chem. 2005, 15(43):4629-4638.

104 Annenkov, V. V., S. V. Patwardhan, D. Belton, E. N. Danilovtseva und C. C. Perry A new stepwise synthesis of a family of propylamines derived from diatom silaffins and their activity in silicification, Chem. Commun. 2006(14):1521-1523. 
105 Lutz, K., C. Gröger, M. Sumper und E. Brunner Biomimetic silica formation: Analysis of the phosphate-induced self-assembly of polyamines, Phys Chem Chem Phys 2005, 7:2812-2815.

106 Knecht, M. R. und D. W. Wright Amine-Terminated Dendrimers as Biomimetic Templates for Silica Nanosphere Formation, Langmuir 2004, 20(11):4728-4732.

107 Hildebrand, M., E. York, J. I. Kelz, A. K. Davis, L. G. Frigeri, D. P. Allison und M. J. Doktycz Nanoscale control of silica morphology and three-dimensional structure during diatom cell wall formation, Journal of Materials Research 2006, 21(10):2689-2698.

108 Hildebrand, M. Diatoms, Biomineralization Processes, and Genomics, Chemical Reviews 2008, 108(11):4855-4874.

109 Sumper, M. Biomimetic patterning of silica by long-chain polyamines, Angew Chem Int Ed Engl 2004, 43(17):2251-2254.

110 De Stefano, C., C. Foti, A. Gianguzza und S. Sammartano Speciation of low molecular weight ligands in natural fluids: protonation constants and association of open chain polyamines with the major components of seawater, Anal. Chim. Acta 2000, 418(1):43-51.

111 De Robertis, A., C. Foti, O. Giuffre und S. Sammartano Dependence on ionic strength of polyamine protonation in $\mathrm{NaCl}$ aqueous solution, J. Chem. Eng. Data 2001, 46(6):1425-1435.

112 Bencini, A., A. Bianchi, E. Garcia-Espana, M. Micheloni und J. A. Ramirez Proton coordination by polyamine compounds in aqueous solution, Coord. Chem. Rev. 1999, 188:97-156.

113 Perry, C. C., D. Belton und K. Shafran in Silicon Biomineralization, Vol. 33 (Ed.: W. E. G. Müller), Springer: Berlin, 2003, pp. 269-299.

114 Kröger, N. und M. Sumper in Biomineralization - From Biology to Biotechnology and Medical Application, 1 ed. (Ed.: E. Baeuerlein), Wiley-VCH: Weinheim, 2000, pp. 151-170.

115 Behrens, P., M. Jahns und H. Menzel in Handbook of Biomineralization (Eds.: P. Behrens, E. Baeuerlein), Wiley VCH: Weinheim, 2007, pp. 3-18.

116 Patwardhan, S. V., N. Mukherjee, M. Steintz-Kannan und S. J. Clarson Bioinspired synthesis of new silica structures, Chem Commun (Camb) 2003(10):1122-1123.

117 Lenoci, L. und P. J. Camp Self-assembly of peptide scaffolds in biosilica formation: computer simulations of a coarse-grained model, J Am Chem Soc 2006, 128(31):10111-10117. 
118 Murthy, V. S., S. B. Kadali und M. S. Wong Polyamine-Guided Synthesis of Anisotropic, Multicompartment Microparticles, ACS Applied Materials \& Interfaces 2009, 1(3):590-596.

119 Rana, R. K., V. S. Murthy, J. Yu und M. S. Wong Nanoparticle self-assembly of hierarchically ordered microcapsule structures, Advanced Materials 2005, 17(9):1145-+.

120 Murthy, V. S., R. K. Rana und M. S. Wong Nanoparticle-assembled capsule synthesis: formation of colloidal polyamine-salt intermediates, J Phys Chem B 2006, 110(51):25619-25627.

121 Patwardhan, S. V., N. Mukherjee und S. J. Clarson Formation of fiber-like amorphous silica structures by externally applied shear, Journal of Inorganic and Organometallic Polymers 2001, 11(2):117-121.

122 Rodriguez, F., D. D. Glawe, R. R. Naik, K. P. Hallinan und M. O. Stone Study of the chemical and physical influences upon in vitro peptide-mediated silica formation, Biomacromolecules 2004, 5(2):261-265.

123 Jung, J. H., Y. Ono und S. Shinkai Sol-gel polycondensation of tetraethoxysilane in a cholesterol-based organogel system results in chiral spiral silica, Angewandte Chemie-International Edition 2000, 39(10):1862-+.

124 Jung, H. J., Y. Ono und S. Shinkai Novel Silica Structures Which Are Prepared by Transcription of Various Superstructures Formed in Organogels, Langmuir 2000, 16:1643-1649.

125 Yuan, J. J. und R. H. Jin Fibrous crystalline hydrogels formed from polymers possessing a linear poly(ethyleneimine) backbone, Langmuir 2005, 21(7):31363145.

126 Yuan, J. J., P. X. Zhu, N. Fukazawa und R. H. Jin Synthesis of nanofiber-based silica networks mediated by organized poly(ethylene imine): Structure, properties, and mechanism, Advanced Functional Materials 2006, 16(17):2205-2212.

127 van Bommel, K. J. C. und S. Shinkai Silica Transcription in the Absence of a Solution Catalyst: The Surface Mechanism, Langmuir 2002, 18(12):4544-4548.

128 Patwardhan, S. V., N. Mukherjee und S. J. Clarson Effect of process parameters on the polymer mediated synthesis of silica at neutral $\mathrm{pH}$, Silicon Chemistry 2002, $1(1): 47-54$.

129 Robinson, D. B., J. L. Rognlien, C. A. Bauer und B. A. Simmons Dependence of amine-accelerated silicate condensation on amine structure, J. Mater. Chem. 2007, 17:2113.

130 Menzel, H., S. Horstmann, P. Behrens, P. Bärnreuther, I. Krueger und M. Jahns Chemical properties of polyamines with relevance to the biomineralization of silica, Chem. Commun. 2003, 24:2994-2995. 
131 Bazzicalupi, C., et al. Thermodynamics of phosphate and pyrophosphate anions binding by polyammonium receptors, J. Am. Ceram. Soc. 1999, 121(29):68076815.

132 Patwardhan, S. V., S. J. Clarson und C. C. Perry On the role(s) of additives in bioinspired silicification, Chemical Communications 2005(9):1113-1121.

133 Laugel, N., J. Hemmerle, C. Porcel, J. C. Voegel, P. Schaaf und V. Ball Nanocomposite silica/polyamine films prepared by a reactive layer-by-layer deposition, Langmuir 2007, 23(7):3706-3711.

134 Patwardhan, S. V. und S. J. Clarson Silicification and biosilicification, Silicon Chemistry 2002, 1(3):207-214.

135 Zollfrank, C., H. Scheel und P. Greil Regioselectively ordered silica nanotubes by molecular templating, Advanced Materials 2007, 19(7):984-+.

136 Yeh, Y. Q., B. C. Chen, H. P. Lin und C. Y. Tang Synthesis of hollow silica spheres with mesostructured shell using cationic-anionic-neutral block copolymer ternary surfactants, Langmuir 2006, 22(1):6-9.

137 Kinnunen, P. K. J. und J. M. Holopainen Mechanisms of initiation of membrane fusion: Role of lipids, Bioscience Reports 2000, 20(6):465-482.

138 Israelachvili, J., Intermolecular \& surface forces, 3 ed., Academic Press: London, 1992.

139 Israelachvili, J. N., D. J. Mitchell und B. W. Ninham Theory of Self-Assembly of Hydrocarbon Amphiphiles into Micelles and Bilayers, Journal of the Chemical Society-Faraday Transactions Ii 1976, 72:1525-1568.

140 Gruner, S. M., P. R. Cullis, M. J. Hope und C. P. S. Tilcock Lipid Polymorphism the Molecular-Basis of Nonbilayer Phases, Annual Review of Biophysics and Biophysical Chemistry 1985, 14:211-238.

141 Cullis, P. R., M. J. Hope und C. P. S. Tilcock Lipid Polymorphism and the Roles of Lipids in Membranes, Chem. Phys. Lipids 1986, 40(2-4):127-144.

142 Lopez, O., A. de la Maza, L. Coderch, C. Lopez-Iglesias, E. Wehrli und J. L. Parra Direct formation of mixed micelles in the solubilization of phospholipid liposomes by Triton X-100, FEBS Lett 1998, 426(3):314-318.

143 Vinson, P. K., Y. Talmon und A. Walter Vesicle-micelle transition of phosphatidylcholine and octyl glucoside elucidated by cryo-transmission electron microscopy, Biophys J 1989, 56(4):669-681.

144 Israelachvili, J. N., D. J. Mitchell und B. W. Ninham Theory of Self-Assembly of Lipid Bilayers and Vesicles, Biochimica Et Biophysica Acta 1977, 470(2):185-201. 
145 MacDonald, R. C., R. I. MacDonald, B. P. Menco, K. Takeshita, N. K. Subbarao und L. R. Hu Small-volume extrusion apparatus for preparation of large, unilamellar vesicles, Biochim Biophys Acta 1991, 1061(2):297-303.

146 Crommelin, D. J. A. Influence of Lipid Composition and Ionic Strength on the Physical Stability of Liposomes, Journal of Pharmaceutical Sciences 1984, 73(11):1559-1563.

147 Carmona-Ribeiro, A. M. Synthetic Amphiphile Vesicles, Chem. Soc. Rev. 1992, 21(3):209-214.

148 Tsuruta, L. R. und A. M. Carmona-Ribeiro Counterion effects on colloid stability of cationic vesicles and bilayer-covered polystyrene microspheres, J. Phys. Chem. 1996, 100(17):7130-7134.

149 Cistola, D. P., J. A. Hamilton, D. Jackson und D. M. Small Ionization and Phase Behavior of Fatty Acids in Water: Application of the Gibbs Phase Rule, Biochemistry 1988, 27:1881-1888.

150 Cevc, G. Membrane Electrostatics, Biochim. Biophys. Acta, Rev. Biomembr. 1990, 1031(3):311-382.

151 Kleinschmidt, J. H. und L. K. Tamm Structural Transitions in Short-Chain Lipid Assemblies Studied by 31P-NMR Spectroscopy, Biophysical Journal 2002, 83:9941003.

152 Helmecke, O., C. Menneking, P. Behrens und H. Menzel Influence of shape and surface properties of microstructured reaction areas on the deposition of silica, Colloid Polym. Sci. 2008, 286(3):305-311.

153 Yuan, J. J. und R. H. Jin Multiply shaped silica mediated by aggregates of linear poly(ethyleneimine), Advanced Materials 2005, 17(7):885-888.

154 Lopez, P. L., Gautier, C., Livage, J., Coradin, T. Mimicking biogenic silica nanostrucutres formation, Current Nanoscience 2005, 1:73-83.

155 Xu, M., G. M. Gratson, E. B. Duoss, R. F. Shepherd und J. A. Lewis Biomimetic silicification of $3 D$ polyamine-rich scaffolds assembled by direct ink writing, Soft Matter 2006, 2(3):205-209.

156 Dickerson, M. B., K. H. Sandhage und R. R. Naik Protein- and Peptide-Directed Syntheses of Inorganic Materials, Chem. Rev. 2008, 108(11):4935-4978.

157 Foo, C. W., J. Huang und D. L. Kaplan Lessons from seashells: silica mineralization via protein templating, Trends Biotechnol. 2004, 22(11):577-585.

158 Wetherbee, R. The diatom glasshouse, Science 2002, 298(5593):547-547.

159 Ptak, M., M. Egret-Charlier, A. Sanson und O. Bouloussa A NMR study of the ionization of fatty acids, fatty amines and $N$-acylamino acids incorporated in 
phosphatidylcholine vesicles, Biochim. Biophys. Acta, Biomembr. 1980, 600(2):387-397.

160 Cistola, D. P., D. M. Small und J. A. Hamilton Carbon 13 NMR Studies of Saturated Fatty Acids Bound to Bovine Serum Albumin II. Electrostatic Interactions in Individual Fatty-Acid Binding-Sites, J. Biol. Chem. 1987, 262(23):10980-10985.

161 Brzezinski, M. A., R. J. Olson und S. W. Chisholm Silicon Availability and CellCycle Progression in Marine Diatoms, Mar. Ecol. Prog. Ser. 1990, 67(1):83-96.

162 Stöber, W., A. Fink und E. Bohn Controlled Growth of Monodisperse Silica Spheres in Micron Size Range, J. Colloid Interface Sci. 1968, 26(1):62-\&.

163 Lootens, D., C. Vautrin, H. Van Damme und T. Zemb Facetted hollow silica vesicles made by templating catanionic surfactant vesicles, Journal of Materials Chemistry 2003, 13(9):2072-2074.

164 Hentze, H. P., S. R. Raghavan, C. A. McKelvey und E. W. Kaler Silica hollow spheres by templating of catanionic vesicles, Langmuir 2003, 19(4):1069-1074.

165 Dufrene, Y. F., W. R. Barger, J. B. D. Green und G. U. Lee Nanometer-scale surface properties of mixed phospholipid monolayers and bilayers, Langmuir 1997, 13(18):4779-4784.

166 Schwartz, D. K., J. Garnaes, R. Viswanathan und J. A. N. Zasadzinski Surface Order and Stability of Langmuir-Blodgett-Films, Science 1992, 257(5069):508511.

167 Hui, S. W., R. Viswanathan, J. A. Zasadzinski und J. N. Israelachvili The Structure and Stability of Phospholipid-Bilayers by Atomic-Force Microscopy, Biophys. J. 1995, 68(1):171-178.

168 Richter, R., A. Mukhopadhyay und A. Brisson Pathways of lipid vesicle deposition on solid surfaces: a combined QCM-D and AFM study, Biophys. J. 2003, 85(5):3035-3047.

169 Stelzle, M., G. Weissmuller und E. Sackmann On the Application of Supported Bilayers as Receptive Layers for Biosensors with Electrical Detection, J. Phys. Chem. 1993, 97(12):2974-2981.

170 Albrecht, O., H. Gruler und E. Sackmann Polymorphism of Phospholipid Monolayers, J. Phys. 1978, 39(3):301-313.

171 Tamm, L. K. und H. M. McConnell Supported phospholipid bilayers, Biophys J 1985, 47(1):105-113.

172 Curatolo, W. The physical properties of glycolipids, Biochimica et Biophysica Acta 1987, 906:111-136. 
173 Hato, M., H. Minamikawa, K. Tamada, T. Baba und Y. Tanabe Self-assembly of synthetic glycolipid / water systems, Advances in Colloid and Interface Science 1999, 80:233-270.

174 Hato, M. und H. Minamikawa The Effects of Oligosaccharide Stereochemistry on the Physical Properties of Aqueous Synthetic Glycolipids, Langmuir 1996, 12:1658-1665.

175 Dörfler, H.-D., C. Koth und W. Rettig Complete and Partial Miscibility in Binary Monolayers of Phosphatidylethanolamines with Different Lengths of Acyl Groups, Langmuir 1995, 11(12):4803-4810.

176 Dörfler, H.-D., C. Koth und W. Rettig Miscibility Behavior of Binary Monolayers in Dependence on the Chain Length Differences and Film States of the Phosphatidylcholine/Phosphatidylethanolamine Mixtures, J. Colloid Interface Sci. 1996, 180(2):478-487.

177 Sanchez, J. und A. Badia Atomic force microscopy studies of lateral phase separation in mixed monolayers of dipalmitoylphosphatidylcholine and dilauroylphosphatidylcholine, Thin Solid Films 2003, 440:223-239.

178 Maget-Dana, R. The monolayer technique: a potent tool for studying the interfacial properties of antimicrobial and membrane-lytic peptides and their interactions with lipid membranes, Biochim Biophys Acta 1999, 1462(1-2):109-140.

179 Insoluble Monolayers at Liquid-Gas Interfaces (Ed.: Gaines, G. L.), J. Wiley and Sons, New York, 1966

180 Gzyl, B. und M. Paluch Properties of insoluble mixed monolayers of lipids at the water/air interface, Progress in Colloid \& Polymer Science 2001, 118:22-26.

181 Park, C. K., F. J. Schmitt, L. Evert, D. K. Schwartz, J. N. Israelachvili und C. M. Knobler Film Balance and Fluorescence Microscopic Investigation of the Effects of Ca2+ on Mixed DMPC/DMPG Monolayers, Langmuir 1999, 15:202-206.

182 Discher, B. M., W. R. Schief, V. Vogel und S. B. Hall Phase separation in monolayers of pulmonary surfactant phospholipids at the air-water interface: compression and structure, Biophys. J. 1999, 77:2051-2061.

183 Ross, M., C. Steinem, H.-J. Galla und A. Janshoff Visualization of Chemical and Physical Properties of Calcium-Induced Domains in DPPC/DPPS LangmuirBlodgett Layers, Langmuir 2001, 17:2437-2445.

184 Kim, D. J., K. B. Lee, Y. S. Chi, W. J. Kim, H. J. Paik und I. S. Choi Biomimetic formation of silica thin films by surface-initiated polymerization of 2(dimethylamino) ethyl methacrylate and silicic acid, Langmuir 2004, 20(19):79047906.

185 Helmecke, O., A. Hirsch, P. Behrens und H. Menzel Microstructured reaction areas for the deposition of silica, Colloid Polym. Sci. 2008, 286(2):225-231. 
186 Brott, L. L., R. R. Naik, D. J. Pikas, S. M. Kirkpatrick, D. W. Tomlin, P. W. Whitlock, S. J. Clarson und M. O. Stone Ultrafast holographic nanopatterning of biocatalytically formed silica, Nature 2001, 413(6853):291-293.

187 Coffman, E. A., A. V. Melechko, D. P. Allison, M. L. Simpson und M. J. Doktycz Surface patterning of silica nanostructures using bio-inspired templates and directed synthesis, Langmuir 2004, 20(20):8431-8436.

188 Kent, M. S., J. K. Murton, F. J. Zendejas, H. Tran, B. A. Simmons, S. Satija und I. Kuzmenko Nanosilica Formation At Lipid Membranes Induced by the Parent Sequence of a Silaffin Peptide, Langmuir 2009, 25(1):305-310.

189 Richter, R. P. und A. R. Brisson Following the formation of supported lipid bilayers on mica: a study combining AFM, $Q C M-D$, and ellipsometry, Biophys $\mathrm{J}$ 2005, 88(5):3422-3433.

190 Hennesthal, C. und C. Steinem Pore-spanning lipid bilayers visualized by scanning force microscopy, J. Am. Chem. Soc. 2000, 122(33):8085-8086.

191 Solon, J., P. Streicher, R. Richter, F. Brochard-Wyart und P. Bassereau Vesicles surfing on a lipid bilayer: Self-induced haptotactic motion, Proc. Natl. Acad. Sci. USA 2006, 103(33):12382-12387.

192 Ziomkowska, J. Georg-August Universität Göttingen (Göttingen), 2009.

193 Higgins, M. J., J. E. Sader, P. Mulvaney und R. Wetherbee Probing the surface of living diatoms with atomic force microscopy: The nanostructure and nanomechanical properties of the mucilage layer, J. Phycol. 2003, 39(4):722-734.

194 Gebeshuber, I. C., J. H. Kindt, J. B. Thompson, Y. Del Amo, H. Stachelberger, M. A. Brzezinski, G. D. Stucky, D. E. Morse und P. K. Hansma Atomic force microscopy study of living diatoms in ambient conditions, J. Microsc. (Oxf) 2003, 212:292-299.

195 Almqvist, N., Y. Delamo, B. L. Smith, N. H. Thomson, A. Bartholdson, R. Lal, M. Brzezinski und P. K. Hansma Micromechanical and structural properties of a pennate diatom investigated by atomic force microscopy, J. Microsc. 2001, 202:518-532.

196 Vrieling, E. G., Q. Sun, M. Tian, P. J. Kooyman, W. W. Gieskes, R. A. van Santen und N. A. Sommerdijk Salinity-dependent diatom biosilicification implies an important role of external ionic strength, Proc Natl Acad Sci U S A 2007, 104(25):10441-10446.

197 Noll, F., Sumper, M., Hampp, N. Nanostrucure of diatom silica surfaces and of biomimetic analogues, Nano Letters 2002, 2(2):91-95.

198 Losic, D., R. J. Pillar, T. Dilger, J. G. Mitchell und N. H. Voelcker Atomic force microscopy (AFM) characterization of the porous silica nanostructure of two centric diatoms, J. Porous Mater. 2007, 14(1):61-69. 
199 Losic, D., K. Short, J. G. Mitchell, R. Lal und N. H. Voelcker $A F M$ nanoindentations of diatom biosilica surfaces, Langmuir 2007, 23(9):5014-5021.

200 Hildebrand, M., M. J. Doktycz und D. P. Allison Application of AFM in understanding biomineral formation in diatoms, Pflugers Arch. 2008, 456(1):127137.

201 Crawford, S. A., M. J. Higgins, P. Mulvaney und R. Wetherbee Nanostructure of the diatom frustule as revealed by atomic force and scanning electron microscopy, J. Phycol. 2001, 37(4):543-554.

202 Losic, D., G. Rosengarten, J. G. Mitchell und N. H. Voelcker Pore architecture of diatom frustules: Potential nanostructured membranes for molecular and particle separations, J. Nanosci. Nanotechnol. 2006, 6(4):982-989.

203 Schmid, A. M. M. und D. Schulz Wall Morphogenesis in Diatoms - Deposition of Silica by Cytoplasmic Vesicles, Protoplasma 1979, 100(3-4):267-288.

204 Wallace, A. F., J. J. DeYoreo und P. M. Dove Kinetics of Silica Nucleation on Carboxyl-and Amine-Terminated Surfaces: Insights for Biomineralization, J. Am. Chem. Soc. 2009, 131(14):5244-5250. 



\section{Anhang}

\subsection{Berechnungen zum Säure-Base-Verhalten der Polyamine und Aminolipide}

Die Software SPARC (http://ibmlc2.chem.uga.edu/sparc/) wurde für die Berechnung von $\mathrm{pK}_{\mathrm{s}}-$ Werten eingesetzt.

\section{Polyamine}

Zunächst wurde für jede Verbindung ermittelt, welche Protonierungsverteilungen in den Molekülen in einem pH-Bereich von $-0,2$ bis 14 auftreten. Dabei wird für jeden pH-Wert die genaue Verteilung von unterschiedlich protonierten Molekülen ausgegeben (Abbildung 9.1). Daraus wurde das genaue Ladungsmuster der bei pH 6,8 (bei diesem pH-Wert wurden alle Experimente durchgeführt) am häufigsten vorliegenden Struktur ermittelt. In Abbildung 9.1, in der die Ergebnisse für C2N6 aufgetragen sind, ist dies die dargestellte Verbindung, deren Verteilung durch die rote Linie repräsentiert wird.

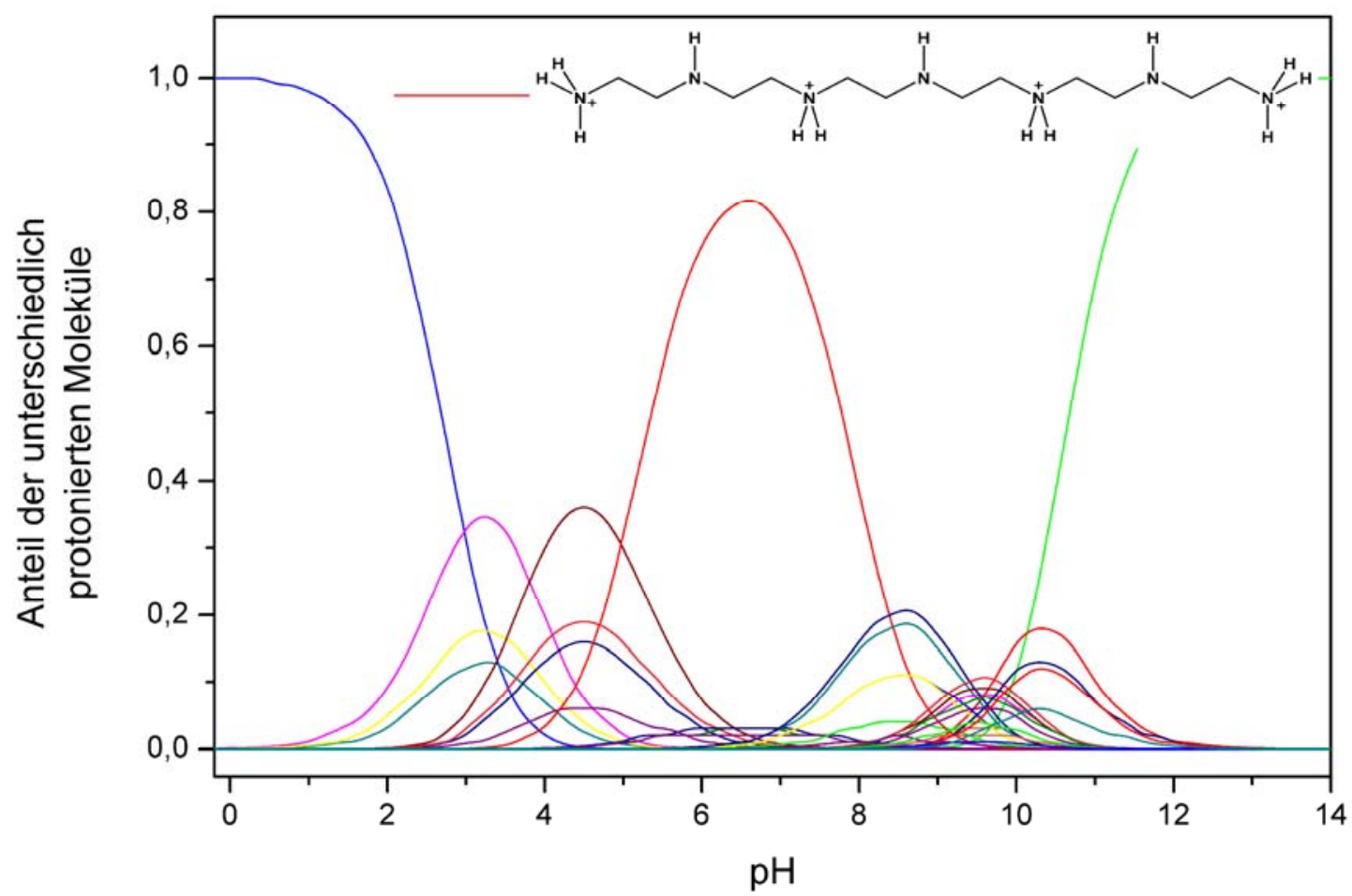

Abbildung 9.1 Anteil der unterschiedlich protonierten Moleküle in Abhängigkeit vom pH-Wert für das Polyamin C2N6 ermittelt mit SPARC. Die Struktur und die Ladungsverteilung der bei pH 6,8 am häufigsten auftretenden Struktur ist dargestellt. Deren Auftreten wird durch die rote Linie wiedergegeben. 
Weiterhin wurde für pH 6,8 die mittlere Protonierung aller vorliegenden Strukturen ermittelt, indem für jede die Ladung und der Anteil einkalkuliert wurde und ein Gesamtwert berechnet wurde. Im Fall von C2N6 beispielsweise (Abbildung 9.1) sind durchschnittlich $56 \%$ aller Aminogruppen bei $\mathrm{pH}$ 6,8 positiv geladen.

Da die Dauer der Berechnungen mit steigender Kettenlängen der Polyamine zunahm und die Anfrage vom Server nach einer gewissen Zeit unterbrochen wurden, konnten sie nicht für alle in der Arbeit verwendeten Polyamine durchgeführt werden. Um trotzdem die mittlere Protonierung und die wahrscheinlichste Ladungsverteilung ermitteln zu können, wurden bis zur maximal möglichen Kettenlänge, wie oben beschrieben, die Daten bestimmt. Im Anschluss wurde die mittlere Ladung $z$ gegen die jeweilige Anzahl an Aminoeinheiten pro Molekül $n_{\mathrm{N}}$ aufgetragen. Dies ist in Abbildung 9.2 für die Propylamine, die Butylamine sowie für $N$-methylierte Proplyamine gezeigt. Es stellte sich heraus, dass die Ladung in allen Fällen proportional zu $n_{\mathrm{N}}$ ist. Durch lineare Anpassung der Daten und Extrapolation konnte die mittlere Ladung auch für die längeren Polyamine berechnet werden. Zudem wurde die Ladungsverteilung durch Vergleich mit den kürzeren Polyaminen abgeschätzt.
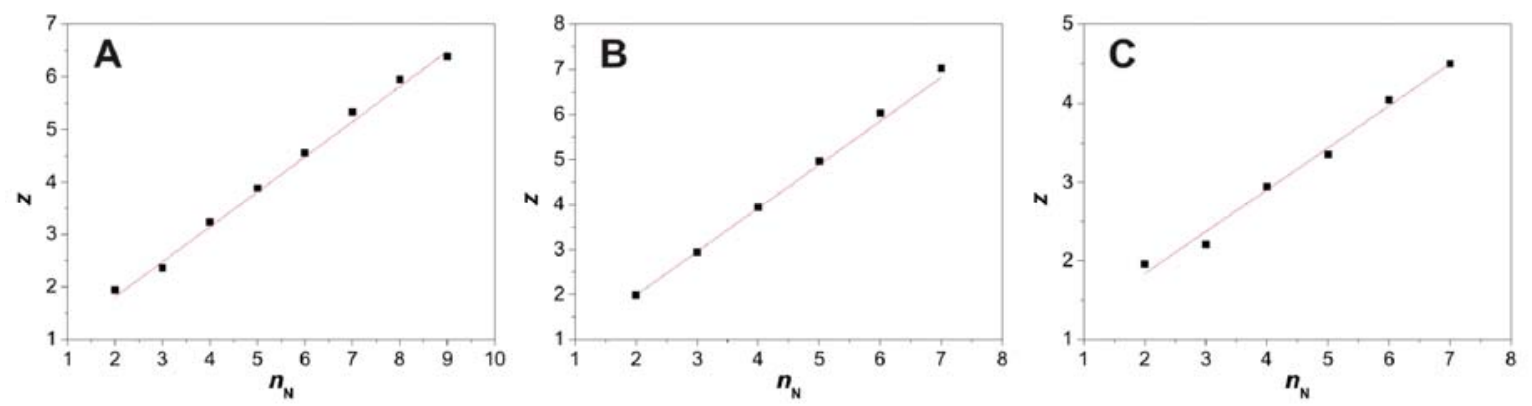

Abbildung 9.2 Ladung $z$ aufgetragen gegen die Anzahl an Aminoeinheiten pro Molekül $n_{\mathrm{N}}$ für Polyamine. A Propylamine, B Butylamine und C $N$-methylierte Propylamine. Die rote Linie zeigt jeweils das Ergebnis der linearen Anpassung der Daten.

\section{Aminolipide}

Die Berechnungen zum Säure-Base-Verhalten der Aminolipide erfolgten analog den für die Polyaminen beschriebenen. Hier gestaltete sich die Auswertung einfacher, da außer beim Lysinollipid keine makroskopische Analyse nötig war und aufgrund der kleinen Anzahl an Aminogruppen pro Molekül jede Substanz vollständig mit Hilfe von SPARC charakterisiert werden konnte. Der pH-Wert, bei dem die Protonierung ermittelt wurde, ist pH 6,0, da die Experiment bei diesem durchgeführt wurden. In Abbildung 9.3 ist die 
Auswertung für das tertiäre Aminolipid dargestellt, das bei pH 6,0 eine mittlere Ladung von 0,2 aufweist.

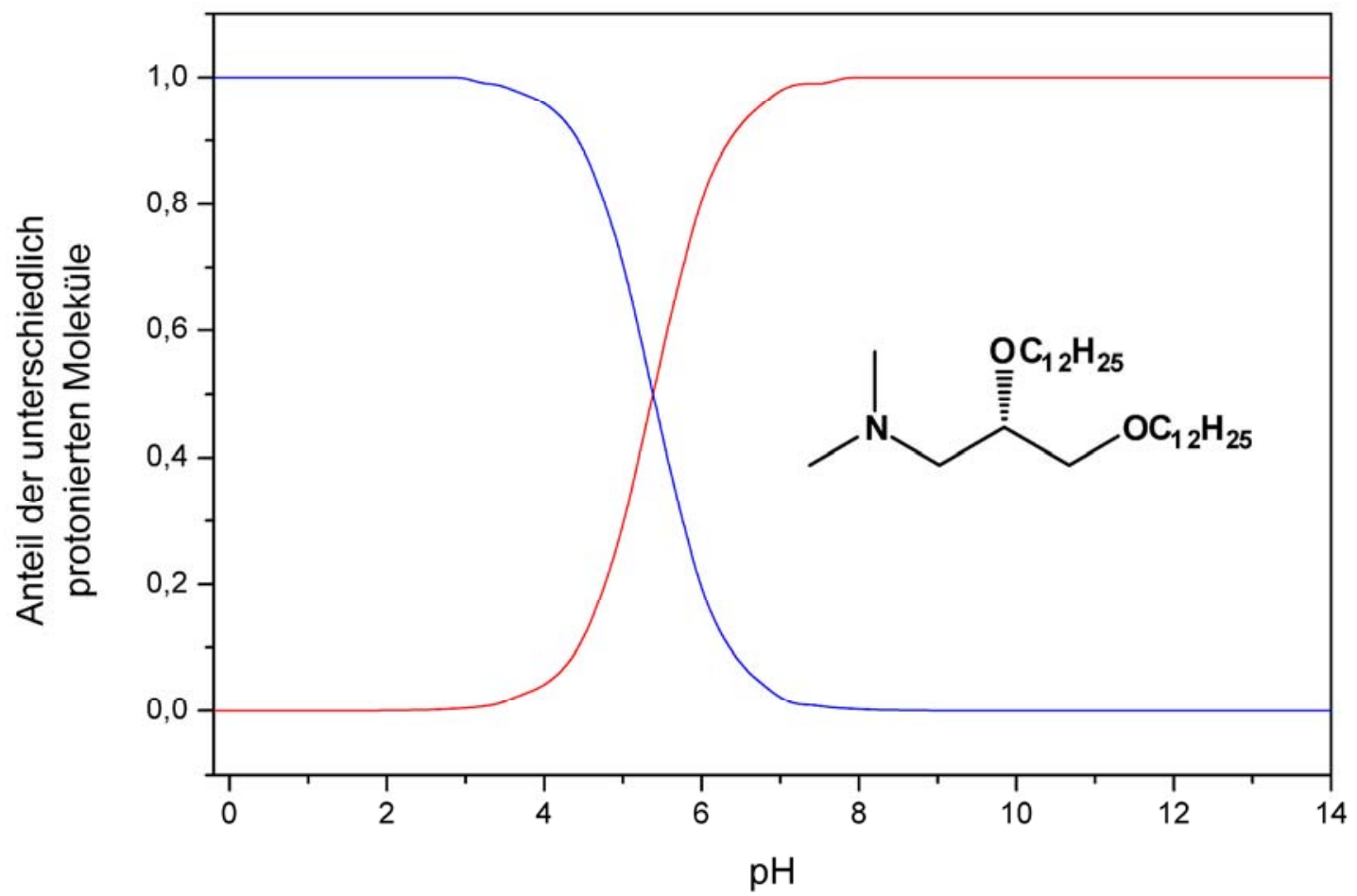

Abbildung 9.3 Anteil der unterschiedlich protonierten Moleküle in Abhängigkeit vom pH-Wert für das tertiäre Aminolipid ermittelt mit SPARC. Die Struktur mit Ladungsverteilung der bei pH 6,0 am häufigsten auftretenden Struktur ist dargestellt. Deren Auftreten wird durch die rote Linie wiedergegeben.

\subsection{Zur Schichtdickenbestimmung mittels Ellipsometrie verwendete Modelle}

Im einfachsten Modell wird angenommen, dass mehrere planare homogene Schichten übereinander liegen (Abbildung 9.4 A), deren Brechungsindizes folgende Werte haben:

$$
\begin{aligned}
& \text { Puffer }\left(n^{*}=1,3328-i 0,0000\right) \\
& \text { SiO }_{2}\left(n^{*}=1,4610-i 0,0000\right)
\end{aligned}
$$

Lipidschicht $\left(n^{*}=1,4884-i 0,0000\right)$

$$
\mathbf{S i O}_{2}\left(n^{*}=1,4610-i 0,0000\right)
$$$$
\text { Si }\left(n^{*}=4,0000-i 0,0538\right)
$$

Hierbei wird angenommen, dass das präzipitierte Silica denselben Brechungsindex aufweist, wie $\mathrm{SiO}_{2}$ auf dem Siliciumwafer. 
A

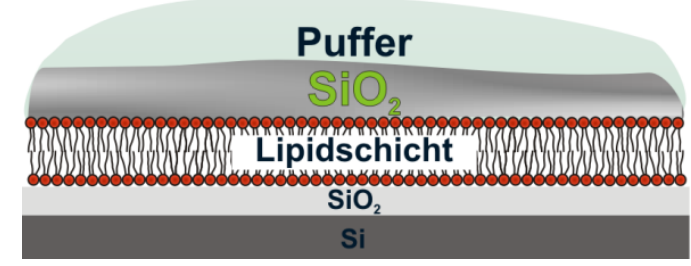

B

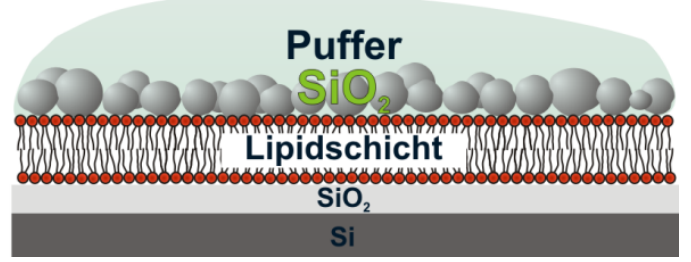

Abbildung 9.4 A Einfaches Schichtmodell für einen Silicafilm präzipitiert auf einer Lipidschicht, die auf einem Silciumwafer mit Siliciumoxidschicht immobilisiert wurde. B Präziseres Modell der Silicaoberfläche, die sich aus Silicapartikel zusammensetzt.

AFM-Aufnahmen des Silicapräzipitats zeigten jedoch keine glatte, sondern vielmehr eine unebene Oberfläche, aufgebaut aus einer Vielzahl runder Partikel (Abbildung 9.4 B). Aus diesem Grund wurde eine Berechnung mittels ,Effektiv-Medien-Theorie‘ (BruggemanModell) durchgeführt, um die tatsächlich vorliegende Oberflächenbeschaffenheit treffender beschreiben zu können. In dem Modell nach Bruggeman wird das effektive Medium als eine totalsymmetrische Anordnung der beiden Medien Silica und Puffer betrachtet:

$$
\varepsilon=\frac{1}{2}\left(-z^{2}+\sqrt{-z^{2}+2 \varepsilon_{2} \varepsilon_{1}}\right)
$$

mit der Permittivität des Gesamtsystems ع, den Permittivitäten von Gastmaterial und umgebendem Medium $\varepsilon_{1}$ und $\varepsilon_{2}$ sowie:

$$
z=\frac{1}{2}\left((3 f-2) \varepsilon_{1}+(1-3 f)\right)
$$

mit dem Volumenanteil $f$.

Genauere Untersuchungen in Rahmen einer Diplomarbeit ${ }^{192}$ zeigten, dass die Annahme eines Volumenverhältnisses Silica/Puffer $=60 / 40$ sinnvoll ist. Durch die Berechnungen ergab sich für Silica ein Brechungsindex von 1,4093. Die Werte der Brechungsindizes der anderen Schichten ( $\mathrm{Si}, \mathrm{SiO}_{2}$, Lipid, Puffer) entsprechen den oben aufgeführten. 


\subsection{Abkürzungsverzeichnis}

\begin{tabular}{|c|c|}
\hline AFM & atomic force microscope \\
\hline $\mathrm{BF}$ & bright field \\
\hline BSE & backscattered electrons \\
\hline C16-GL & C16-Glycerol- $\beta$-Lactosid \\
\hline $\mathrm{C} 2 \mathrm{~N} 7$ & Hexaethylheptamin \\
\hline $\mathrm{C} 3 \mathrm{~N} 12$ & Undecapropyldodecamin \\
\hline $\mathrm{C} 3 \mathrm{~N} 12 \mathrm{Me}$ & methyliertes Undecapropyldodecamin \\
\hline C3N18 & Pentadecapropyloctadecamin \\
\hline C3N6 & Pentapropylhexamin \\
\hline C3N7 & Hexapropylheptamin \\
\hline C4N7 & Hexabutylheptamin \\
\hline DF & dark field \\
\hline DLS & dynamische Lichtstreuung \\
\hline DPPC & 1,2-Dipalmitoyl-sn-glycero-3-phosphatidylcholin \\
\hline EDX & energy dispersive $X$-ray \\
\hline FRET & fluorescence resonance energy transfer \\
\hline$g$ & gaseous, gas-analog \\
\hline IQR & Interquartilabstand \\
\hline LB & Langmuir-Blodgett \\
\hline$l_{c}$ & liquid-condensed \\
\hline LCPA & long-chain polyamines \\
\hline$l_{e}$ & liquid-expanded \\
\hline LUV & large unilamellar vesicles \\
\hline LysinolL & Lysinollipid \\
\hline MES & 2-(N-Morpholino)ethansulfonsäure \\
\hline NWI & Nanowizard I \\
\hline NWII & Nanowizard II \\
\hline pAL & primäres Aminolipid \\
\hline PE & Primärelektronen \\
\hline $\mathrm{P}_{\mathrm{i}}$ & anorganisches Phosphat \\
\hline qAL & quartäres Aminolipid \\
\hline$S$ & solid \\
\hline sAL & sekundäres Aminolipid \\
\hline SDV & silica deposition vesicle \\
\hline SE & Sekundärelektronen \\
\hline SEM & scanning electron microscopy \\
\hline SIT & silicon transporter \\
\hline STEM & scanning transmission electron microscopy \\
\hline STM & scanning tunneling microscope \\
\hline
\end{tabular}




$\begin{array}{ll}\text { tAL } & \text { tertiäres Aminolipid } \\ \text { TE } & \text { transmittierte Elektronen } \\ \text { TEM } & \text { transmission electron microscopy } \\ \text { TFA } & \text { Trifluoressigsäure } \\ \text { TMOS } & \text { Tetramethoxysilan }\end{array}$

\subsection{Symbolverzeichnis}

\begin{tabular}{|c|c|}
\hline$A$ & Fläche \\
\hline$a_{0}$ & optimale Fläche der polaren Kopfgruppe \\
\hline$B$ & magnetischer Feldvektor \\
\hline$c$ & Konzentration \\
\hline$c$ & Federkonstante \\
\hline$d$ & Durchmesser \\
\hline$D$ & Diffusionskoeffizient \\
\hline$d$ & Schichtdicken \\
\hline$D$ & Ladungsdichte \\
\hline$d$ & Schichtdicke \\
\hline E & Energie des elektrischen Feldes \\
\hline E & elektrischer Feldvektor \\
\hline$e$ & Elementarladung \\
\hline$E_{\mathrm{p}}$ & elektrisches Feld parallel \\
\hline$E_{\mathrm{p}, 0}$ & Amplitude von $E_{\mathrm{p}}$ \\
\hline$E_{\mathrm{s}}$ & elektrisches Feld senkrecht \\
\hline$E_{\mathrm{s}, 0}$ & Amplitude von $E_{\mathrm{s}}$ \\
\hline$f$ & Volumenanteil \\
\hline$f_{0}$ & Resonanzfrequenz \\
\hline$h$ & Binbreite \\
\hline$h$ & Hydrophobizität \\
\hline$I$ & Intensität \\
\hline$k$ & Geschwindigkeitskonstante \\
\hline$k_{B}$ & Boltzmann-Konstante \\
\hline$l$ & Moleküllänge \\
\hline$l_{\mathrm{c}}$ & kritische Länge \\
\hline$M$ & molare Masse \\
\hline$m$ & Masse \\
\hline$n$ & Anzahl an Ereignissen/Messungen \\
\hline$n$ & Brechungsindex \\
\hline$n$ & Stoffmenge \\
\hline$n^{*}$ & komplexer Brechungsindex \\
\hline
\end{tabular}




\begin{tabular}{|c|c|}
\hline$N_{\mathrm{N}}$ & Anzahl Aminogruppen pro Molekül \\
\hline$P$ & p-Wert \\
\hline$P$ & Packungsparameter \\
\hline$q$ & Betrag des Streuvektors \\
\hline$Q$ & Ladung \\
\hline $\mathrm{Q}_{0,25}, \mathrm{Q}_{0,25}, \mathrm{Q}_{0,75}$ & Quartile \\
\hline$Q_{p}$ & p-Quantile \\
\hline$R$ & Reflektivität \\
\hline$r_{\mathrm{H}}$ & hydrodynamischer Radius \\
\hline$R^{\mathrm{p}}, R^{\mathrm{s}}, r^{\mathrm{p}}, r^{\mathrm{s}}$ & Fresnel-Reflexionskoeffizienten \\
\hline$S_{a}$ & arithmetisches Mittel der 3D-Rauheit \\
\hline$T$ & Temperatur \\
\hline$t$ & Zeit \\
\hline$T$ & Temperatur \\
\hline$T_{m}$ & Phasenumwandlungstemperatur \\
\hline$v, V$ & Volumen \\
\hline $\bar{z}$ & mittlere Molekülladung \\
\hline$\beta$ & optische Weglänge \\
\hline$\Gamma$ & Halbwertsbreite \\
\hline$\gamma$ & Oberflächenspannung \\
\hline$\delta$ & Phasenverschiebung \\
\hline$\Delta$ & ellipsometrischer Winkel \\
\hline$\Delta G_{\mathrm{ex}}$ & Molare freie Exzess-Oberflächenenergie \\
\hline$\varepsilon$ & Dielektrizitätszahl \\
\hline$\varepsilon$ & Permittivität \\
\hline$\eta$ & Viskosität \\
\hline$\theta$ & Winkel \\
\hline$\lambda$ & Wellenlänge \\
\hline$\mu$ & Potential \\
\hline$\pi$ & Oberflächendruck \\
\hline$\sigma$ & Standardabweichung \\
\hline$\tau$ & Abklingzeit \\
\hline$\chi$ & Molenbruch \\
\hline$\Psi$ & ellipsometrischer Winkel \\
\hline$\omega$ & Kreisfrequenz \\
\hline
\end{tabular}




\subsection{Chemikalien}

Ammoniaklösung (25\%)

Ammoniumheptamolybdat

cantilever $\mathrm{CSC} 37$

cantilever MSCT-Au

cantilever Pointprobe NCH

Chloroform, reinst

DPPC

Einwegküvetten Plastikbrand

Eppendorfpipetten

Ethanol, reinst

Glimmerscheiben

Isopropanol, reinst

Kaliumchlorid

MES

Methanol, reinst

Mikroliterspritze

Mucasol ${ }^{\circledR}$

Natriumacetat

Natriumdihydrogenphosphat

Natriumhydrogenphosphat

Natriumhydroxid

Orangegel

Polycarbonatmembranen

Salzsäure

Silberleitlack

Siliciumstandardlösung

Siliziumträger (100 nm Oxid)

Siliziumträger (ohne Oxid)

Sulforhodamin 101 DHPE

Tetramethoxysilan

Wasserstoffperoxid (30\%)

$\beta$-BODIPY 500/510 C12-HPC
VWR International, Darmstadt

Merck

Mikromasch, Tallinn, Estland

Veeco, Camarillo, USA

Nanosensors, Neuchatel, CH

Merck, Darmstadt

Avanti Polar Lipids, Alabaster (USA)

Brand, Wertheim

Eppendorf, Hamburg

Merck, Darmstadt

Plano, Wetzlar

Merck, Darmstadt

AppliChem, Darmstadt

Carl Roth GmbH, Karlsruhe

Merck, Darmstadt

Hamilton, Bonaduz (CH)

Veeco, Camarillo, USA

Merck, Darmstadt

Merck, Darmstadt

Merck, Darmstadt

Merck, Darmstadt

Merck, Darmstadt

Avestin, Ottawa (USA)

Merck, Darmstadt

Conrad Elektronik, Wernberg

Sigma Aldrich

CrysTec, Berlin

Si-Mat, Landsberg/Lech

Sigma-Aldrich, Taufkirchen

Sigma-Aldrich, Taufkirchen

Carl Roth GmbH, Karlsruhe

Invitrogen, Carlsbad (USA) 


\subsection{Geräte}

Filmwaage:

Wilhelmy-Filmwaage mit Filmlift

Riegler \& Kirstein, Potsdam

Fluoreszenzmikroskop

Axiotech Vario

Zeiss, Göttingen

Filtersatz 44 (BP 475/40, FT 500, BP 530/50)

Zeiss, Göttingen

Filtersatz 45 (BP 560/40, FT 585, BP 630/75)

Zeiss, Göttingen

Achroplan 40x/0.8 W Wasserimmersionsobjektiv

Zeiss, Göttingen

EC Epiplan-Neofluar 20x/0.50 DIC M27 Objektiv

Zeiss, Göttingen

Rasterkraftmikroskope

NanoWizard I

JPK Instruments, Berlin

Nanowizard II, BioAFM

JPK Instruments, Berlin

\section{Lichtstreuapparatur}

Eigenbau, Physikalische Fakultät Göttingen

Rasterelektronenmikroskop

Leo Supra 55VP

Zeiss, Oberkochen

Transmissionselektronenmikroskop

Philips CM200-FEG-UT

Ellipsometer

EL X-02C

DRE GmbH, Ratzeburg

UV/Vis-Spektrophotometer

Cary 50 scan

Varian, Darmstadt

Sonstiges

Feinwaage CP 225D

Sartorius, Göttingen

LiposoFast $^{\circledR}$

Avestin, Ottawa (Kanada)

pH-Meter Calimatic 766

Knick, Berlin

Reinstwasseranlage, Milli Elix 5

Millipore, Eschborn

Schüttler Reax Top

Heidolph, Schwabach

Ultraschallbad Sonorex

Bandelin, Berlin

Vakuumtrockenschrank

Binder, Tuttlingen

Zentrifuge Allegra X-22R

Beckman Coulter, Krefeld 



\section{Danksagung}

Mein Dank gilt...

insbesondere Prof. Dr. Claudia Steinem für die Bereitstellung eines interessanten und herausfordernden Themas, für die großen Freiheiten im Rahmen der Doktorarbeit und für die exzellente Betreuung.

Prof. Dr. Franc Meyer für die freundliche Übernahme des Korreferats dieser Arbeit.

Prof. Dr. Armin Geyer sowie Prof. Dr. Manfred Sumper für die anregenden Diskussionen im Rahmen der Kooperation.

Ralph Wieneke für die gute Zusammenarbeit, den Ideenaustausch und nicht zuletzt die Bereitstellung der Verbindungen.

meinen Mitstreiterinnen Joanna Ziomkowska, Svenja Heitmüller und Frauke Müller, die durch ihre hervorragenden Arbeiten einen wesentlichen Beitrag zu dieser Arbeit leisteten. Es hat mir viel Freude bereitet, mit euch zusammenzuarbeiten.

Prof. Dr. Michael Seibt für die Durchführung der TEM- und EDX-Messungen und die damit verbundenen Diskussionen und Erklärungen.

Uta Bete und Antje Krüger für die Einführung und Betreuung am SEM, EDX und XPS.

allen Korrekturlesern für die schnelle und gewissenhafte Bearbeitung.

unseren technischen Angestellten: Hans-Jörg, der mir im Polyamin-Projekt eine umfassende und unentbehrliche Hilfe und immer für jegliche Fachsimpelei offen war. Micha, die mich mit einer unzählbaren Zahl an Überträgen und Filmwaagemessungen unterstützt hat. Jutta, die mir im Laboralltag (und manchmal auch darüber hinaus) mit Rat, Tat und einer helfenden Hand zur Seite stand.

ganz besonders dem gesamten Arbeitskreis, von der Regensburger Anfangsgruppe bis zum jetzigen Göttinger Trupp, nicht nur für das angenehme und anregende Arbeitsklima, sondern natürlich auch für die außerordentlich schöne und lustige Zeit! Allen voran Ruth, meiner langjährigen Labormitbewohnerin, die immer ein offenes Ohr hatte und mit der es einfach ,NichtLustig' war; Eva, die immer aufmunternd und hilfsbereit war und mit der ich auch das gemeinsame WG-Leben entspannt genießen konnte; Barbara, die mich durch 
die gesamte Studienzeit begleitet hat und ohne die diese Zeit lange nicht so schön gewesen wäre; Gretel und Ines, die ich zwar erst später kennengelernt habe, deswegen aber nicht weniger vermissen werde, genau wie alle anderen.

all meinen Freunden und Mitbewohnern aus Regensburg und Göttingen für eine wunderschöne Zeit.

Tobi, Barbara, Linda, Christian, Sussan und Zabine für die lustige Studienzeit und die Freundschaft, die mit Ende des Studiums zum Glück nicht beendet war.

meinen Spezln aus' $m$ Moos, Sabine, Marion und Sandra. Ich freue mich darüber, so besondere Freunde zu haben, die mich immer wieder herzlich empfangen.

Familie Edler nicht nur für den Druck des Einbands, sondern auch für alles andere.

meiner gesamten Familie, die mir Rückhalt gibt und zu der ich immer gerne heimkomme. ganz besonders meinen Eltern für einfach Alles! Wie ihr mich unterstützt ist nicht selbstverständlich und ich weiß das sehr zu schätzen. 




\section{Posterpräsentationen}

'Silaffin-Analogues in Artificial Membrane-Systems'

International Workshop 'Dynamics of artificial and biological membranes'

März 2006, Gomadingen

'Formation of ordered silica structures via membrane templating'

Jahrestagung der Deutschen Biophysikalischen Gesellschaft

September 2006, Mainz

'Formation of ordered silica structures via membrane templating'

3rd International Symposium on Complex Materials

März 2007, Kerkrade/NL

'Bio-inspired silica formation via membrane templating'

6th European Biophysics Congress (EBSA)

Juli 2007, London/GB

'Membrane templates for biomimetic silica formation'

International Workshop 'mechanical and electrical properties of artificial and cellular membranes'

April 2008, Gomadingen

,Membrantemplate für die biomimetische Bildung von Silica-Strukturen ‘

Göttinger Chemie-Forum

Juli 2008, Göttingen

'Membrane templates for biomimetic silica formation'

Jahrestagung der Deutschen Biophysikalischen Gesellschaft

Oktober 2008, Berlin 



\section{Lebenslauf}

Anja Christina Bernecker

geboren am 11. April 1979 in Neuburg an der Donau

Staatsangehörigkeit: deutsch

\section{Schulbildung}

09/1985 - 07/1990 Volksschule Königsmoos

09/1990 - 06/1999 Descartes-Gymnasium Neuburg a.d. Donau

25.06.1999

Allgemeine Hochschulreife

\section{Studium}

10/1999-03/2006 Studium der Chemie an der Universität Regensburg

05.03.2002 Diplomvorprüfung im Fach Chemie

05/2005 - 01/2006 Diplomarbeit an der Arbeitsgruppe von Prof. Dr. Claudia Steinem am Institut für Analytische Chemie, Chemo- und Biosensorik der Universität Regensburg,

Thema: Silaffin-Analoga in artifiziellen Membransystemen:

Phasenverhalten und $\mathrm{SiO}_{2}$-präzipitierende Eigenschaften

31.01 .2006

Diplom im Fach Chemie, Schwerpunktfach Medizinische Chemie

\section{Dissertation}

03/2006 - 09/2009 Dissertation am Institut für Organische und Biomolekulare Chemie, Georg-August-Universität Göttingen

Betreuerin: Prof. Dr. Claudia Steinem 